




\section{Inhalt von Lieferung 3.}

Prstı, Oтro: Hydrobiologische Studien über Ostalpenseen. (Mit

1 Karte [Taf. VII] und 19 Abbildungen auf Taf. VIII) . . 385-596

\section{An die Herren Mitarbeiter!}

Jeder Autor erhält von seinem Beitrage 50 Sonderabdrücke gratis geliefert, weitere Abdrücke werden zu Selbstkosten berechnet. Die Textabbildungen werden womöglich in sauberer schwarzer Tuschzeichnung die Beschriftung jedoch nur in Bleistift - um $1 / 8$ größer erbeten, als sie bei der Wiedergabe erscheinen sollen.

Die Herren Mitarbeiter werden gebeten, sich in ihrer Darstellung so knapp und kurz zu halten, sowie Tabellen und Abbildungen soweit zu beschränken, als es ohne Beeinträchtigung ihrer Arbeiten möglich ist. Korrekturen sind auf das Aeußerste zu beschränken. Es ist beabsichtigt, die Archivhefte in kürzeren Zeitabständen erscheinen zu lassen, soweit es die Aufnahmefähigkeit der Abonnenten 'gestattet, um die vorliegenden und einlaufenden Arbeiten so schnell als möglich zu publizieren. Die Herren Verfasser werden gebeten, Nachsicht uiben zu wollen, falls der Abdruck ihrer Arbeiten nicht immer mit der erwünschten Schnelligkeit sollte erfolgen können.

Verlag und Schriftleitung des Archivs für Hydrobiologie. 


\title{
P4) Hydrobiologische Studien über Ostalpenseen.
}

\author{
Von Otto Pesta.
}

(Veröffentlicht mit Unterstützung aus der Czermakstiftung der Akademie der Wissenschaften in Wien.)

Mit 1 Karte (Taf. VII) und 19 Abbildungen auf Taf. VIII.

\section{Einleitung.}

\begin{abstract}
„Die Probleme, die sich den Planktologen im Laufe der Arbeit aufgedrängt haben, sind sehr vielgestaltig, und da dieser Forschungszweig aueh noch recht jung ist, sind ganz abgeklärte und umfassende Ergebnisse noch nicht zu erwarten; denn wie auf keinem anderen Gebiete mufs hier eine Unsumme von Beobachtungen von allen Orten zusammengetragen werden, bevor es möglich sein wird, auf Grund dieser Detailstudien Schlufffolgerungen von allgemeiner $\mathrm{Be}$ deutung zu erzielen."
\end{abstract}

Aus F. Ruttner: „Die Verteilung des Planktons im Süf̧wasser." In Fortschritte d.naturwiss. Forschung (Abderhalden), vol. X, p. 273.

Die Bearbeitung des Crustaceenmateriales einiger Planktonnetzfänge, das vor 12 Jahren aus hochgelegenen Alpenseen in Kärnten und Tirol aufgesammelt war, und der mächtige Einfluß, den alsbald das Bekanntwerden mit Fr. ZschoкKE's Schrift über die „Tierwelt der Hochgebirgsseen" und die daran anschließende Fachliteratur auszuüben begann, ließ den Wunsch entstehen, an der hydrobiologischen Untersuchung alpiner Süßwasserbecken in irgendeiner Richtung selbst teilzuhaben; der Verwirklichung des Vorsatzes kam eine angeborene Begeisterungsfähigkeit für die Bergwelt entgegen. Das Streben richtete sich zunächst darauf, im Bereiche der Ostalpen eine Reihe von Seen, deren absolute Höhenlage sich oberhalb der Waldgrenze, also in der Hochgebirgsregion im strengen Sinne, befand, nicht nur in bezug auf ihre Copepoden- und Cladocerenfauna hin zu untersuchen, sondern auch ihre Milieueigenheiten festzustellen,-- um hierdurch einheitliche Daten zur Beantwortung der Frage žu gèwinnen, ob der Nachweis einer gesetzmäßigen Abhängigkeit zwischen dem Vorkommen einzelner Faunenelemente aus dem geologisch-petrographischen Charakter der Umgebung des betreffenden Fundortes möglich wäre. Hiezu waren vor allem 
chemische Analysen der Seewässer notwendig. Der Ausbruch des Krieges hatte den begonnenen Arbeiten Einhalt geboten; sie konnten erst im Jahre 1918 wieder aufgenommen und leider nur im bescheidenen Maße fortgesetzt werden. Inzwischen waren tiergeographische Probleme und insbesondere die Frage der vielgenannten Eiszeitrelikte in den Vordergrund wissenschaftlicher Abhandlungen gerückt. Der Mangel an Kenntnissen von Fundorten aus dem östlichen Teil der Alpen machte sich fühlbar (oder wurde übersehen!) und half dadurch mit, einer spekulativen Forschungsrichtung größeren Raum zu geben. Dieser Mangel entsprang nicht allein tatsächlichen Lücken in der Untersuchung ostalpiner Seebecken als auch zum guten Teile der Unzugänglichkeit zahlreicher Literaturbelege sowie dem Fehlen einer zusammenfassenden Übersicht von Fundorten des genannten Bereiches. In diesem Sinne zur Kenntnis der Verbreitung zweier wichtiger Gruppen tierischer Seenbewohner - den Copepoden und Cladoceren - beizutragen, schien eines Versuches wert, der nunmehr in diesen „Studien“ vorliegt. Zu den bewußten Unvollständigkeiten, welche ihr Inhalt aufweist, wird eine kritische Prüfung vielleicht noch manchen Nachweis unbewußter Mängel hinzufügen können. Wenn die Darstellung des Stoffes jedoch dazu geeignet ist, weiteren Untersuchungen und Beobachtungen a uf dem Gebiete der Hydrobiologie der Ostalpenseen als Grundlage und Anregungzu dienen, so erreicht siedamitihren Haupt$\mathrm{z}$ w e c k. Vielleicht ist es auch gelungen, die bekanntlich wenig gut gestimmte Meinung über den wissenschaftlichen Wert der Faunenlisten in diesem Rahmen zu einem besseren Urteil zu veranlassen.

Die Akademie der Wissenschaften in Wien hat die Ausführung meiner eigenen Untersuchungen, deren Ergebnisse in diesen "Studien“ enthalten sind, durch Zuwendung einer einmaligen Subvention aus dem Legate ScHolz und einer ebensolchen aus der Erbschaft Czermak gefördert; desgleichen bewilligten das vormalige Oberstkämmereramt und das Bundesministerium für Unterricht Unterstützungen und Urlaube zu diesem Zweck. Ferner nahmen sich meiner Arbeiten durch bereitwilligst gewährte Erfüllung verschiedener Wünsche und durch mehrfache Auskünfte in entgegenkommender Weise an: Prof. Dr. V. Brenм (Eger), Prof. Dr. K. von Dalda Torre (Innsbruck), Deutscher Fischereiverein (Berlin), Prof. Dr. O. Haempel (Wien), Hofrat A. Handirssch (Wien), Kustos Dr. K. Holdhaus (Wien), Regierungsrat Direktor Hans Huber (Innsbruck), Prof. H. Klein (Wien), Hofrat Prof. Dr. Lorenz-Liburnau 
(Wien), Meteorologische Zentralanstalt (Wien), Prof. Dr. R. Moxtr (Pavia), Regierungsrat Dr. A. Penther (Wien), Prof. Dr. Th. Pesta (Wien), Dr. F. Rutrmer (Lunz), Hofrat Dr. Fr. Steindachyer $†$ (Wien), Prof. Dr. A. Steuer (Innsbruck), Ingenieur Dr. E. Walther (Wien), Hofrat Prof. Dr. R. Wettstein - Westersheim (Wien). Zoologischbotanische Gesellschaft (Wien). - Allen genannten Behörden, Instituten und Personen gebührt es, an dieser Stelle meinen Dank auszusprechen. Im besonderen bin ich den Herren Prof. H. KLEIN und Ingenieur Dr. E. Walther für die Durchführung der Wasseranalysen und meinem Bruder Prof. Dr. Th. Pesta für die Begleitung im Hochgebirge und seine vielseitige Hilfe bei der Aufsammlung des Materiales und der Aufnahme topographischer Daten zu Dank verpflichtet.

W i e n, im November 1922.

\section{Der Verfasser.}

Der Druck des Manuskriptes wurde durch Zuwendung eines namhaften Kostenbeitrages seitens der Akademie der Wissenschaften in Wien ermöglicht; es ist mir eine angenehme Pflicht, für diese außerordentliche Förderung öffentlich danken zu können. Großes Entgegenkommen bewies mir die E. Schweizerbart'sche Verlagsbuchhandlung in Stuttgart, welche die Drucklegung in geradezu mustergültiger Weise und allen meinen Wünschen entsprechend durchführte; ich spreche ihr Anerkennung und Dank aus!

W i e n, im Juli 1923.

Der Verfasser. 


\section{Inhalt.}

I. A b s c h n it t: Beiträge zur hydrobiologischen Untersuchung einiger Hochgebirgsseen im Gebiete der Ostalpen

II. A b s chn itt: Die chemische Beschaffenheit des "Wassers einiger Hochgebirgsseen der Ostalpen . . . . . .

III. A b s c h n it t: Geographisch-geologische Charakteristik, Begrenzung und Einteilung der Ostalpen. Seenverzeichnis.

IV. A b s chnitt: Beiträge zur hydrobiologischen Charakteristik der Ostalpenseen (197) unter Berücksichtigung ihrer Copepoden- und Cladocerenfauna (Spezieslisten)

V. A b s chnitt: Úber die Verbreitung und das Auftreten der Copepoden und Cladoceren in den Seen der Ostalpen

$545-586$

VI. A b s chnitt: Literaturnachweise $587-595$ 


\section{Abschnitt.}

\section{Beiträge zur hydrobiologischen Untersuchung einiger Hochgebirgsseen im Gebiete der Ostalpen.}

Über die Hochgebirgsseen in Tirol und ihre Fauna wurde an anderer Stelle [Verhandlungen d. Zool.-Botan. Gesellsch. Wien 1912 p. 158, 1914 p. 210, 1915 p. 227 und 1918 p. (269)] in drei Beiträgen und einem Vortrage berichtet. Die Fortführung dieser Untersuch ngen konnte erst im Jahre 1918 wieder aufgenommen werden; es war jedoch infolge der gänzlich veränderten äußeren Umstände notwendig geworden, die geplanten Arbeiten zunächst nicht mehr in Tirol, sondern in anderen Ostalpengebieten zu versuchen. Der westliche Teil des Zuges der $\mathrm{N}$ i e der en Ta u er n, soweit derselbe innerhalb der Grenzen Obersteiermarks fällt, schien hiezu in mehrfacher Weise sehr geeignet; während der Sommermonate des Jahres 1918 und 1919 wurden hier Aufsammlungen aus sieben Wasserbecken gewonnen. Noch mehr wie in Tirol waren die Kenntnisse über die aquatile Fauna der Seen dieses Bereiches als mangelhafte zu bezeichnen. Im Anschlusse daran entfielen während des folgenden Jahres 1920 auf die Gruppe der Hohen T a u e n hydrographisch-faunistische Beobachtungen an zwei Seen, welche im Salzburger Gebiete des Großglocknerstockes liegen. Erst im Jahre 1921 führten gleichartige Untersuchungen über zwei Hochgebirgsseen der Tuxeralpen in das ursprünglich gewählte Arbeitsgebiet Tirol zurück. Von den gesamten elf Wasserbecken gehört nur der Grünsee im Stubachtale der Waldregion an, während sich alle übrigen durchwegs oberhalb der Waldgrenze befinden. Die Richtlinien, nach welchen die hydrographischen und faunistischen Daten gewonnen wurden, waren dabei die nämlichen geblieben, wie sie für die eingangs zitierten „Beiträge" Anwendung gefunden hatten. Von dem Gedanken geleitet, daß jeder See gerade durch die genauere Kenntnis seiner Lage und Umgebung, seiner Ufer- und Bodenbeschaffenheit, wie seiner allgemeinen Vegetationsverhältnisse, der Art und Zahl seiner Zuflüsse usf. erst deutlich in seiner Individualität hervortritt, schien uns keine Beobachtung zu wertlos. Soweit es möglich war, wurde auch je eine Wasserprobe (1 Liter Oberflächenwasser) zur chemischen Analyse entnommen. In diesem Rąhmen 
dürften die nicht immer im besten Ansehen stehenden Spezieslisten mehr zu sagen haben als sonst.

Vor der Beantwortung der Frage, welche Wasserbecken den Hochgebirgsseen zuzurechnen sind, soll zunächst auf die übliche, von $\mathrm{O}$. HeER [vergl. Heller 1881] stammende Gliederung des Gesamtgeländes verwiesen werden; demnach sind folgende Zonen (Regionen) angenommen worden:

0 - $650 \mathrm{~m}$ über dem Meere, Seen der Talregion;

$$
\begin{aligned}
& 650-1200, \quad, \quad, \quad, \quad, \quad, \quad, \text {, unteren Waldregion; } \\
& 1200-1700, \text {, " , , , , , oberen Waldregion; } \\
& 1700-2300, \quad, \quad \text {, , , , , , alpinen Region; } \\
& 2300-2700, \quad, \quad \text {, , , , , , subnivalen Region; } \\
& 2700-4000, \quad, \quad, \quad, \quad, \quad, \quad \text {, nivalen Region (bezw. } \\
& 3900 \mathrm{~m} \text { über dem Meere, Ortler, } \\
& \text { als Höchstgipfel der Ostalpen). }
\end{aligned}
$$

Eine Einreihung der Wasserbecken nach diesen feststehenden Schranken kann den tatsächlichen hydrobiologischen Verhältnissen naturgemäß nicht entsprechen. Es wird vielmehr notwendig sein, die Scheidung nach einem Faktor vorzunehmen, welcher vor wiege $\mathrm{nd}$ die allgemeine $n$ klimatischen Bedingungen bestimmt und beherrscht. Ein solcher ist im Alpenbereich durch den Verlauf der Linie des geschlossenen Waldbestandes in ausgeprägtem Maße gegeben. An keine fixierten, absoluten Höhenlagen gebunden, sondern ganz den örtlichen Eigenheiten nachgebend, nimmt die Waldgrenze in dẻn Ostalpen den Gürtel zwischen 1700-2000 m ein; sie reicht also nicht so weit aufwärts wie durchschnittlich in den Westalpen. Ähnlich wie sich der landschaftliche Charakter nach dem Überschreiten der Waldgrenze unvermittelt ändert, so drücken hier auch tiefgreifend neue klimatische Eigenschaften den Wasserbecken ihren Stempel auf. Niederschläge und Temperatur, Zuflüsse und Uferbildung, Bodenbeschaffenheit und aquatile Flora lassen am Hochgebirgssee oberhalb der Waldgrenze ausgeprägte Merkmale erkennen; dazu gesellt sich nicht zuletzt die chemische Beschaffenheit des Wassers, welches ganz allgemein als besonders ,rein“ bezeichnet werden muf. [Vergl. dazu Abschnitt II dieser Studien.] Die Summe aller dieser gemeinsamen Milieubedingungen wird auch der Wasserfauna einen bestimmten, in den großen Zügen gleichartigen Charakter verleihen, welcher am dominierenden Bestandteil, den Copepoden und Cladoceren, vor allem beobachtet werden kann. In diesem Sinne wird von uns als $\mathrm{Hoch}$ gebirgssee jedes perennierende (der periodischen Aus- 
trocknung nicht unterworfene), o berhal b der W a l dgrenze ge e le ge n e (ohne Rücksicht auf die m-Zahl der absoluten Höhenlage über dem Mecresspiegel) Wa s serbe cken bezeichnet. Gegen über den in niedereren Höhenschichten, d. h. innerhalb des Waldbestandes befindlichen Seen ist der Hochgebirgssee durch eine vielsehwächerentwickelte makroskopische Wasservegetation und urcheine verhältnismäBiggeringe Artenzahl an Copepoden und Cladocerengekennzeichnet und stellt somit auch einen biologisch gut umsehriebenen Seet y pus dar. Aus dem Gesagten geht deshalb hervor, daß der von uns gebrauchte Ausdruck „Hochgebirgssee“ sich wesentlich von den gleichen Bezeichnungen anderer Autoren unterscheidet; wir trennen von ihm alle übrigen stehenden Gewässer, für welche im Bereiche der Alpen der Name „Gebirgssee“ gewählt werden soll.

In letzter Zeit wurde von zwei Seiten der Versuch unternommen, eine Gruppierung der Seen von hydrobiologischen Gesichtspunkten aus durchzuführen, so von E. Naumann [1918] und von A. Thienemann [1922]; während der erste Autor „in einem Griff dem Zusammenhang zwischen geographischer und geologischer Lage der Gegend, biologischer Art bezw. der sonstigen Beschaffenheit der Gewässer und endlich dem Ernährungstypus des Entomostrakenplanktons im Vergleich mit dem Gesamtstoffhaushalt des Wassers" (op. cit. p. 38) Rechnung tragen will und nach diesen allgemeinen Prinzipien eine Dreiteilung (1. Seen reich an Phytoplankton nebst organischem Peritripton einer vorwiegend planktogenen Herkunft; 2. Seen arm an Phytoplankton bezw. planktogenem Detritus, reich an allochthonem Peritripton; 3. Seen arm an Phytoplankton ebenso wie an Peritripton jeglicher Art) vornimmt, unterscheidet Thienemann hauptsächlich nach der Verschiedenheit der Sauerstoffverhältnisse bezw. nach dem hierdurch bedingten Vorkommen von Tanytarsus-Coregonus oder Chironomus-Non Coregonus zwei Typen (1. den Subalpinen Seetypus und 2. den Baltischen Seetypus). Der von uns vorhin als Hochgebirgssee charakterisierte Typus würde - den bisherigen Beobachtungen zufolge - weder in der Einteilung Naumann's noch in jener von Thienemann befriedigend Platz finden, obwohl er sicherlich mit der „3. Seengruppe“ des ersten Autors viel gemein hat und andererseits auch im "Subalpinen See-Typus" ThieneMANN's vielleicht als ein besonders gekennzeichnetes Extrem aufgezählt werden könnte. Es scheint uns aber, daß die von Naumans sowohl wie 
von Thienemans aufgestellten Gewässertypen doch nicht ausreichend sein werden, um auf die Gebirgs- und Hochgebirgsseen der Alpen allgemeine Anwendung $\mathrm{zu}$ finden; gerade im Bereiche der Ostalpen steht der Zahl der heute schon hydrobiologisch besser bekannten größeren Randseen eine bedeutend größere Anzahl gänzlich unerforschter oder nur cinseitig untersuchter Wasserbecken der mannigfaltigsten Art gegenüber, welche sich von den Randseen wesentlich unterscheiden.

Als Merkbiätter für die zur faunistischen Beurteilung der Wasserbecken wertvoll erschieinenden Angaben wurde von uns eine „Aufnahmstabelle $^{\text {z }}$ zusammengestellt, welche dem Arbeitsplane für unsere Untersuchungen im Hocl gebirge entsprechen und die Gleichartigkeit der Aufzcichnungen für jeden See gewährleisten sollte. Sie enthält folgende Punkte in folgender Anordnt:ng:

1. Name. 2. Datum. 3. Zcit der Beobachtung. 4. Höhenlage ü d. M. 5 Größe des Wasserbcckens. 6. Lufttemperatur. 7. Besonnung. 8. Uferbeschaffenheit. 9. Oberfläch. Wassertemperatur. 10. Wasserflora. 11. Mineralogische Beschaffenheit des Seegrundes. 12. Tiefe. 13. Geologischpetrograplischer Charakter der Umgebung. 14. Zuflüsse. 15. Zuflußwassertemperatur. 16. Topographische Skizze nebst Bercerkungen über Wasserfärbung, periocische Austrocknung, Strömungen, Eisbildung, Steinschlag, Fischbestand usw.

\section{Der Filzsee. (Textfig. 1.)}

(Besuchsdatum: 17. August 1918.)

Höhe über dem Meeresspiegel: ca. $2100 \mathrm{~m}$.

L a g e u n d U m g e bu n g: Nächst der Station Aich (Obersteiermark) nimmt die Enns den ungefähr in der Süd-Nordrichtung abfließenden Seewigbach auf; sein Quellgebiet, der nach Norden gerichtete Hang des Hochwildstellstockes, beherbergt neben einer Anzahl anderer Secbccken den sogenannten Filzsee am Fuße des Höchsteingipfels. Das Secbett stellt eine Mulde von Geröll und Schutt dar, dessen Umrandung im Süden und Westen steil aufsteigende, aus Fels- und Steintrümmern bestehende Hänge bildet, während gegen Norden und Osten mehr oder weniger flache Moosböden von geringer Ausbreitung den Uferrand einnehmen. Der See selbst war zur Zeit des Besuches vollkommen schneeund eisfrei, nur an schattenseitig gelegenen Stellen des steinigen Südufers zeigten sich geringe Schneereste; von hier empfängt das Becken auch zwei schwache, aus dem Geröll hervorbrechende Rinnsale als Zuflüsse. Ein dritter Zufluß mündet in den Nordzipfel des Sees. Das Gebiet 
liegt durchaus im Granitgneis; Kalke fehlen auch auf den umgebenden Gipfe'n.

Gr ö B e: Die Flächenausdehnung des kleinen Wasserbeckens beträgt etwa 100 Schritte in der Länge und 40 in der Breite.

Beschaffenheit des Seebodens. Wasserflora: Das dunkelgrün gefärbt erscheinende Wasser ist klar und läßt überall den mit Felstrümmern bedeckten Grund erkennen, der an seinen tiffsten Stellen höchstens wenige Nieter erreichen mag, in Ufernähe vorwiegend 80-100 cm, nur an einzelnen Punkten 1,5-2 m Tiefe aufweist. Im übrigen bedeckt den Boden ein fein zerricbener, mineralischer Detritus, der auch in den entnommenen Planktonnetzfängen als „Schlamm“ wahrgenommen werden konnte. Eine makroskopisch sichtbare Vegetation fehlt so gut wie vollkommen; nur ein minimaler Algenbelag an den unter Wasser liegenden Steinen kam zur Beobachtung.

Wassertemperatur: Dieselbe betrug in der Zeit zwischen $9^{\mathrm{h}} 45-11^{\mathrm{h}}$ a. m. an der Oberfläche $7^{\circ} \mathrm{C}$. Für die drei Zuflüsse wurden zur gleichen Zeit $3^{0}, 3,2^{0}$ und $4,5^{0} \mathrm{C}$ gemessen. (Vgl. dazu die Topograph. Skizze, Zuflüsse a, b und c.) [Lufttemperatur um $10^{\text {h }}$ a. m. $14^{0} \mathrm{C}$ !]

Clad oceren - und Copepodenfauna: Die Planktonnetzfänge erwiesen sich als äußerst arten- und individuenarm; sie enthielten in größerer Menge Larvenstadien von Copepoden und die Cladocere Acroperus harpae BAIRD, dagegen zurücktretend Exemplare von Cyclops serrulatus Fischer, Cyclops sp. und Chydorus sp.

\section{Der Obere Giglachsee.}

(Besuchsdatum: 9. August 1919.)

Höh e über dem lieeresspiegel: ca. 1960 m [Giglachseehütte $2005 \mathrm{~m}$ ].

L a g e u n d U m g e b u n g: Die Schladminger Tauern beherbergen am Fuße der Engelkaarspitze und des Hadinggipfels den großen, langgestreckten, an seinem Abflußende in Buchten gegliederten, Unteren Giglachsee $(1945 \mathrm{~m})$ und den den Talschluß gegen den Znachsattel einnehmenden, bedeutend kleineren, ungegliederten Oberen Giglachsee; etwa $50 \mathrm{~m}$ höher als die genannten Wasserbecken steht an der Anstiegseite des dem Hadinggipfel gegenüber sich erhebenden Stockes der Kampspitzen die Giglachseeł. ütte des D.-Ö. Alpenvereines. Die Topographie des ganzen Gebietes und seiner Umgebung wurde in vorzüglicher Weise von Joseph Schattauer [Mitteilg. D.Ö.AV. 1914, Nr. 8, p. 113-116] und J. V. JäCKLE [Zeitschrift d. D.Ö.AV. 1916, vol. 47, p. 37-40] 
behandelt. Der Obere See liegt in einer offenen, während der Sommermonate Juli und August durchschnittlich 10-12 Stunden voller Bcsonmung ausgesetzten flachen Mulde. Mit Ausnahme der Südostseite, wo Geröllhalden vom Hadingkamm bis zum See reichen, wird er von flachen Ufern umrandet, welche begrasten Almboden mit verstreut umherliegenden Felsblöcken bilden. Es konnten fünf Zuflüsse beobachtet werden, von denen drei im Süden des Sees einmünden, zwei dem Nordwestufer zuströmen; zwei der erstgenannten brechen etwa bloß 6 bis 10 Schritte rom See entfernt aus dem Gerölle des Hadingspitzenhanges hervor. Der mäßig breite Abflußbach zum Unteren Giglachsee befindet sich am Nordostufer. Die Geologie des Niedernschlagsgebietes ist insofern eigenartig, als sich längs dem Anstiege zum vorhin erwähnten Znachsattel eine ungewöhnlich scharf ausgeprägte Scheidung zwischen dem sogenannten Urgestein (Vetternspitzen mit Hadinggipfel als Vorberg) und dem Kalkgestein (Zug der Lungauer- und Steirischen Kalkspitze) bemerkbar macht, die dem Beobachter schon von weitem durch die Verschiedenheit in der Vegetation auffällt. Der See selbst liegt im Schieferbereich (Radstätter Quarzite).

Größe: Die Wasserfläche hat eine Ausdehnung von etwa 500 Schritten in der Länge und etwa 200 Schritten in der Breite.

Beschaffenheit des Seebodens. Wasserflora: Die im allgemeinen gering geneigten Uferränder zeigen steinigen Seegrund; die Bodenflächen werden dazwischen von stark zerkleinertem, mineralogischem Detritus eingenommen und nur selten finden sich Stellen von schlammartiger Beschaffenheit. Am Westrande des Wasserbeckens können in einer Entfernung von $2-5 \mathrm{~m}$ rom Ufer steil abfallende Partien beobachtet werden, die in tiefere Bodenmulden hinabreichen; sie bieten besonders günstige Punkte für den Planktonnetzfang. Die Färbung des Wasserspiegels war am Besuchstage dunkelgrün; gegen Abend ( $5^{\text {h }} 30^{\prime}$ p. m.) stellte sich lebhafter Wellengang in der Richtung von Nordost nach Südwest ein. An Algen erwies sich der See sehr reich; nicht nur, daß diese schon makroskopisch als Überzüge an allen Steinen wahrzunehmen waren, sondern es konnte auch bei der Untersuchung der Planktonnetzfänge ein stark entwickeltes Phytoplankton nachgewiesen werden, welches in Klümpchen- und Fadenalgen und in zahlreichen einzelligen Formen vorhanden war. Das Vorkommen höherer Wasserpflanzen wurde an keiner Stelle beobachtet.

Wassertemperatur: Dieselbe betrug um $5^{\text {h }} 30^{\prime} \mathrm{p} . \mathrm{m}$. an der Oberfläche (im Schatten) 9,5-10 $\mathrm{C}$ [bei gleichzeitiger Lufttemperatur 
von $12,2^{\circ} \mathrm{C}$ ]. Die Wassertemperatur der Zuflüsse am Südostufer des Sees betrug $8^{\circ} \mathrm{C}$ (beim längsten Rinnsal in etwa sechs Schritten Entfernung vom Seeufer) bezw. $3^{0}$ und $2^{0} \mathrm{C}$ (an den Ursprungsstellen der zwei kurzen Zuflüsse). J. V. JäckLE fand den See im Mai (? 1915) noch vollständig zugefroren.

Cladoeeren- und Copepodenfauna: Die vom Ufer aus nahe an der Wasseroberfläche ausgeführten Netzfänge enthielten zahlreiche Nauplien- und Copepoditstadien, die im Leben eine blaßrote Farbe besaßen, sowie Hüllenreste von Ephippialeiern, während in den Tiefenfängen von einigen Metern die Jugendformen der Copepoden auffallend schwach vertreten waren, die Anzahl der Eier sich hingegen vermehrte. Eine Probe aus der Seichtwasserzone nächst einem Zuflusse lieferte neben einigen Copepodenlarven und Eiern sowie zwei eiertragenden Cyclops-Weibchen zahlreiche braungefärbte Chydorus-Exemplare. Es sei erwähnt, daß der See Fische enthält, die nach Größe und Färbung vermutlich als Saiblinge (Salvelinus alpinus L.) anzusprechen sind. Es wurden im ganzen folgende Spezies nachgewiesen: Acroperus harpae BAIRD, Alona affinis var. ornata StINGELIN, Bosmina coregoni BAIRD ser. longispina RüHe, Chydorus sphaericus O. F. MüLLER, Cyclops serrulatus Fischer, Cyclops vernalis Fischer und ein unbestimmter Harpacticide. Zum Vorkommen ron Bosmina sei bemerkt: In den Oberflächenfängen weit seltener vertreten als in jenem aus Tiefen von einigen Metern; die Exemplare (우) zeigen durchweg die für coregoni typische derbe Bedornung der Endkralle des Abdomens, und zwar sitzen vier Zähne auf der Endkralle selbst, zwei auf dem Krallenträger; eine distale feine Strichelung der Kralle konnte nicht konstatiert werden, hingegen ist die doppelte Knickung derselben deutlich ausgeprägt. Die Tiere gehören ohne Zweifel zur Reihe longispina im Sinne RüHE's [1912]; eine vollständige Übereinstimmung mit einer von den 41 hierher fallenden Formen ließ sich nicht feststellen; am nächsten zu stehen kommt B. $c$. var. sempacensis BURckHARDT und $B$. $c$. var. cisterciensis RüHE. Am abgebildeten Exemplar (Textfig. 3) wurden folgende Maße beobachtẹt:

Absolute Körperlänge $680 \mu$. Schalenlänge (umgerechnet auf 1000 Körperlänge) 676. Schalenhöhe (Körperhöhe) 735; die höchste Stelle des Dorsalrandes ist deutlich vor der Mitte der Längsachse des Tieres gelegen. Auge groß; beim abgebildeten Exemplar wohl infolge der Präparation verzogen $(0=37)$. Mucro ziemlich kurz, ca. 37; Inzisuren fehlen. Erste Antenne gleichmäßig schwach gekrümmt, ihr Endteil mit 17 Inzisuren. 
Chemische Zusammensetzung des Wassers: Das Wasser war klar und farblos, sein Geruch sehr schwach, seine Reaktion neutral. Schwebebestandteile zahlreich. Organische Substanz nicht ermittelt (mit Rücksicht auf die Wassermenge von 1 1). Der Verdampfungsrückstand (1 l) hatte das Gewicht von $33 \mathrm{mg}$. Der Glührïckstand wurde nicht bestimmt. $\mathrm{Si}_{2} 1,8 \mathrm{mg}, \mathrm{Al}_{2} \mathrm{O}_{3}+\mathrm{Fe}_{2} \mathrm{O}_{3} 7,6 \mathrm{mg}$, $\mathrm{Ca} O \mathrm{O} 9 \mathrm{mg}, \mathrm{Ng} \mathrm{O} 1,3 \mathrm{mg}, \mathrm{S} \mathrm{O}_{4}$ in Spuren; $\mathrm{Cl}, \mathrm{N} \mathrm{O}_{3}, \mathrm{~N} \mathrm{O}_{2}$ fehlen vollständig. Natrium und Kalium war qualitativ nachweisbar. Die freie $\mathrm{C}_{2}$ (in Spuren) wie auch die gebundene Kohlensäure konnte dem Gewichte nach infolge der geringen Wassermenge nicht ermittelt werden.

\section{Der Kampspitzsee.}

(Besuchsdatum: 10. August 1919.)

Höh e über de m Meeresspiegel: ca. $2200 \mathrm{~m}$.

Lage und Umgebung: Dieser See liegt am Fuße des steil sich auftürmenden Kampspitzenstockes, welcher dem vorhin erwähnten Hadinggipfel im Westen gegenübersteht. Sein Westufer reicht unmittelbar an die jäh abfallenden Geröllhalden der kammartig emporragenden Gipfelreihe (2400 m) heran, im Norden bilden Felsabstürze, an der Sücund Südostseite abgerundete, begraste Kuppen die Umrandung, welche zur Zeit der Untersuchung noch mächtig ausgedehnte Schneefelder bcsaß. Letztere erreichten mit Ausnahme der östlich gelegenen Abflußzone durchwegs den Wasserspiegel des Sees, der in einem Ausmaße von fast drei Viertel seiner Fläche eine Eisdecke trug. Als Zufluß wurde ein unter dem Schneefeld des Westufers laufendes Rinnsal konstatiert; der Abfluß befindet sich am gerade gegenüberliegenden See-Ende und führt sein Wasser dem Giglachbach zu. Das Gebiet liegt im Sehiefer (Radstätter Quarzite).

Grö B e: Der See ist etwa 300 Schritte lang und durchschnittlich halb so breit.

Beschaffenheit des Seebodens. Wasserflora: Das äußerst klare, in dunkelgrüner Farbe erscheinende Wasser gestattet eine deutliche Sicht bis zum steinigen, in geringer Entfernung vom Ufer ungefähr $3 \mathrm{~m}$ tiefen Seegrund, dessen Detritus auffällig glimmerige Beschaffenheit besitzt. Von einer Wasservegetation ist makroskopisch nichts zu sehen; nur in der Nähe des Abflusses entfaltet sich eine äußerst spärliche Flora von Klümpchenalgen an einzelnen Steinen. Die Untersuchung der entnommenen Planktonproben ergab ebenfalls einen auf- 
fallenden Mangel an pflanzlichen Bestandteilen, während der Gehalt an mineralogischem Detritus stark hervortrat.

W a s sertemperatur: In der Zeit von $8-10^{\text {h }}$ a. m. zeigte das Thermometer trotz der Besonnung der gesamten Seefläche, die auch bis Nittag unter voller Insolation stand, eine Oberflächenwassertemperatur von nur $1,5^{\circ} \mathrm{C}$ (gleichzeitige Lufttemperatur im Schatten $10^{\circ} \mathrm{C}$ ).

Cladoceren- und Copepodenfauna: Alle Planktonnetzfänge zeigten ausgesprochene Inçividuen- und Artenarmut. N€ben einigen Ephippien und Schalen abgestorbener Cladoceren enthielten sie folgende Formen: Alonella nana (BAIRD), Canthocamptus schmeili var. biserialis Micoletzky und Cyclops vernalis Fischer. In Ergänzung einer von C. vas Douwe [1917] gegebenen Tabelle über die Unterscheidungsmerkmale der vier bis jetzt beschricbenen Varietäten von $C$. schmeili MrazeK soll hier erwähnt werden, daß die vorliegenden $\delta^{\circ}$ aus dem Kampspitzsee am Basalgliede des 5. Th. oraxbeines 1 kurzen und 1 langen Dorn besitzen und $d a ß$ die doppelte Dornenreihe an der ventralen Hinterrandseite des 2.-4. Abdominalsegmentes auch beim $q$ vorhanden ist.

Chemische Zusammensetzung des Wassers: Das Wasser war klar, farb- und gerucklos, seine Reaktion neutral. Schwebebestandteile gering. Organische S.bstanz mit Rücksicht auf die Wassermenge (1 l) nicht ermittelt. Das Gewicht des Verdampfungsrückstandes betrug $18,6 \mathrm{mg}$. Glüh.rückstand nicht bestimmt. $\mathrm{Si}_{2} 2,4 \mathrm{mg}, \mathrm{Al}_{2} \mathrm{O}_{3}$ $+\mathrm{Fe}_{2} \mathrm{O}_{3} 2,6 \mathrm{mg}$, Ca O 6,8 mg, $\mathrm{Mg} \mathrm{O} 0,53 \mathrm{mg}, \mathrm{S} \mathrm{O}_{4}$ in Spuren, Cl in Spuren; $\mathrm{N} \mathrm{O}_{3}, \mathrm{~N} \mathrm{O}_{2}$ fehlen vollständig. Natrium und Kalium konnten qualitativ nackgewiesen werden. Die freie wie gebundene Kohlensäure wurde dem Gewicht nach nicht bestimmt.

\section{Der Obere Klaftersee.}

(Besuchsdatum: 7. August 1919.)

Höh e über dem Meeresspiegel: ca $2000 \mathrm{~m}$.

Lage und Umgebung: Der obere Klaftersee, auch Klaffersee bezeichnet, liegt im Ursprungsgebiet eines Seitenbaches (Hohenseebach) des Tales der Großen Sölk, welche zwischen Öblarn und Schladming in die Enns (rechtes Ufer) mündet; er ist vom abgeschiedenen Talorte Sankt Nikolai aus im Aufstiege zum Gipfel des Großen Knallsteins $(2600 \mathrm{~m}) \mathrm{zu}$ erreichen. Das Wasserbecken selbst fällt in die Zone der mächtig ausgedehnten Glimmerschiefer, während die Höhen des Knallsteins von körnigen Kalken eingenommen werden, geologisch interessante Inselbildungen, welche auch in unmittelbarer Umgebung 
von St. Nikolai auftreten. Die Ufer des halbmondförmigen, in einer ziemlich flachen Mulde liegenden Klaftersees bilden im Norden und Westen begraste Felshänge, im Süden und Osten niedrige Riegel, so daß dem Almvieh der unweit befindlichen Kältherbergalpe der Zugang zu dieser „Tränke" leicht möglich ist. Große Strecken des Süd- wie Nordwestufers weisen Versumpfung auf. Dem letzteren gehört die Einmündung zweier stärkerer Zuflüsse an; der Seeabfluß befindet sich am Ostende. Bis auf einen Schneefleck von geringer Ausdehnung (im Nordosten) waren die Ufer vollständig schnee-, der Wasserspiegel gänzlich eisfrei.

Größe: Die Seefläche dürfte ungefähr 300 Schritte Längenausdehnung besitzen, doch konnte eine genaue Abschreitung infolge des ungünstigen Wetters nicht vorgenommen werden.

Beschaffenheit des Seebodens. Wasserflora: Der Grund des Sees macht durchweg den Eindruck von feinsandiger und lehmiger Beschaffenheit, wie er für wenig tiefe Wasserbecken oft charakteristisch ist. Diese geringe Tiefe schafft Raum für eine reiche Entwicklung höherer Wasserpflanzen, welche in einzelnen Buchten aus Schilfbeständen und anderen submersen Phanerogamen bestehen, überall aber auch in üppig wuchernden Algen vorhanden ist. Trotz des regnerischen und nebeligen Wetters war die Färbung des Wassers schön dunkelgrün.

Wassertemperatur: Sie betrug an der Oberfläche $7^{0} \mathrm{C}$, gemessen um $11^{\mathbf{h}}$ a. m.; Zuflußtemperatur $5,8^{\circ} \mathrm{C}$.

Clad o ceren-und Copepodenfauna: In den von uns gesammelten fünf Planktonnetzfängen (Uferfänge), welche glünlich gefärbte Proben lieferten (reiches Phytoplankton, insbesondere Desmidiaceen), dominierten die Cladoceren Alona affinis var. ornata STINGELIN und Chydorus sphaericus O. F. MüLlER; außerdem enthielten sie mehrere Exemplare von Cyclops vernalis Fischer und einige von Alonella excisa (Fischer). Sars hat [Crust. Norway, 1913, vol. 6, p. 44] in die Diagnose und Abbildung von Cyclops lucidulus (=vernalis FISCHER) eine Eigentümlichkeit aufgenommen, welche er zweifellos nur als eine individuelle Variation betrachtet. Er sagt ..., ,the terminal joint of the outer ramus having in some eases 3 spines outside in one or other of the pairs instead of the usual number (2)". Wir konnten die 3-Zahl der Dornen am Außenrande des Exopoditenendgliedes nicht nur am 1. und 4. Beinpaare eines und desselben Exemplares, sondern auch bei allen übrigen Individuen beobachten. Vielleicht handelt es sich hier 
um die Fixierung eines Merkmales bei Formen aus alpinen Fundorten; darauf wäre bei der Untersuchung weiteren Materiales zu achten.

\section{Der Obere Sonntagskarsee. (Textfig. 6.)}

(Besuchsdatum: 17. August 1919.)

Höh e über dem Meeresspiegel: $2036 \mathrm{~m}$.

L a g e u n d U m g e b u n g: Das Sonntagskar, nach welchem dieser See und der folgende benannt sind, bildet in der Form eines großen, von gewaltigen Gipfelreihen umzogenen Kessels das Ursprungsgebiet des Rissachbaches, einem Zufluß des sogenannten Talbaches, welcher bei Schladming das rechte Ufer der Enns erreicht. Die höchste Erhebung der Bergkette repräsentiert das Waldhorn $(2700 \mathrm{~m})$, das sich gerade nach Osten über einen jäh abstürzenden Kamm bis zu einem etwa $1800 \mathrm{~m}$ hoch liegenden Alpenweideland fortsetzt; seine nach Osten gerichteten Ausläufer beherrscht das Kieseck (2678 m), an welches sich die Sonntagskarspitzen anreihen. So erscheint das Wasserbecken nur gegen Norden aus der Umklammerung der Bergriesen befreit, wo seinen Abfluß niedere mit Gras bewachsene Riegel umsäumen. Das Gebiet liegt in den Massen des Glimmerschiefers. Aus den Steilabstürzen, die im Bogen von Südwesten bis Südosten nahezu unmittelbar an das Secufer heranreichen, ergießen sich mehrere zum Teil starke Wasserfälle in den See. Mächtig dicke vereiste Schneemassen bedecken hier noch große Strecken der Geröllhalden und schwimmen zum Teil stark mit Schutt bedeckt als losgebrochene Eisschollen im Becken selbst. Der rundliche Umriß des kesselartigen Sees wird am Nordufer durch eine kleine Halbinselbildung unterbrochen, an welche sich östlich der breite Seeabfluß anschließt.

Größ e: Der Durchmesser der Wasserfläche beträgt ungefähr 300 Schritte.

Beschaffenheit des Seebodens. Wasserflora: Das klare dunkelgrün gefärbte Gewässer besitzt durch wegs steinigen Grund, der bis auf $10-15 \mathrm{~m}$ rom Ufer noch deutlich sichtbar ist. Die Bodenbeschaffenheit ist ausgesprochen glimmer-sandig, nirgends versumpft. Der dem Steilabsturz anstehende Teil des Sees dürfte größere Tiefen bel erbergen, entsprechend einer Fortsetzung des wandartigen Felsabbruches. Eine makroskopisch wahrnehmbare Wasserflora fehlt vollständig; der Inhalt der Planktonnetzfänge enthielt stets viel Sand; der Mangel an Algenbestandteilen dokumentierte sich auch darin, daß die 
Proben nach der Konservierung nur in zwei Fällen und nur ganz schwach grünlich gefärbt waren.

Wassertemperatur: An der Oberfläche wurden zurzeit $9^{\mathrm{h}} 45^{\prime}$ a. m. bei Besonnung $3,2^{\circ} \mathrm{C}$ gemessen; die Wassertemperatur zweier Zuflüsse betrug in einem Falle $2,5^{\circ} \mathrm{C}$, im anderen $7^{\circ} \mathrm{C}$. [Vgl. dazu die Topograph. Skizze, Zufluß a und b.]

Cladoeeren- und Copepodenfauna: Sie erwies sich als äußerst spärlich. Als dominierender Crustaceenbestandteil der Netzfänge wurden Schalenreste einer Cladocere (z. T. mit Ephippien, vermutlich von Acroperus) und zahlreiche, offenbar durch den groben mineralogischen Detritus zerstörte Exemplare von Bosmina nachgewiesen, welche ohne Zweifel schon vor der Konservierung abgestorben waren. Nur zwei Entomostraken wurden in normalem Zustande aufgefunden, nämlich Acroperus harpae BAIRD (in 2 Exemplaren) und Canthocamptus cuspidatus SchMeIL (in zahlreichen Exemplaren). Acroperus harpae stimmt mit der von STingelin [1895] beschriebenen Herbstform überein; die hintere ventrale Schalenecke zeigt keine Zähnchenbildung, hingegen ist der hintere Schalenrand bis zur Dorsalkontur fein "gestrichelt". Die Körperlänge eines o von Canthocamptus cuspidatus betrug $0,68 \mathrm{~mm}$ (ohne Furca!).

Chemische Zusammensetzung des Wassers: Das: Wasser war klar und farblos, von sehr schwachem Geruch, seine Reaktion neutral. Schwebebestandteile gering; organische Substanz nicht ermittelt. Gewicht des Verdampfungsrückstandes $29,25 \mathrm{mg}$ ( $1 \mathrm{ll}$ l). Glührückstand nicht bestimmbar. $\mathrm{Si}_{2} 5,01 \mathrm{mg}, \mathrm{Al}_{2} \mathrm{O}_{3}+\mathrm{Fe}_{2} \mathrm{O}_{3} 1,7 \mathrm{mg}$, $\mathrm{Ca} O \mathrm{O}$ 17,02 mg, $\mathrm{Mg} \mathrm{O} 2,36 \mathrm{mg} ; \mathrm{S} \mathrm{O}_{4}$ in Spuren, ebenso Cl in Spuren; $\mathrm{N} \mathrm{O}_{3}, \mathrm{~N} \mathrm{O}_{2}$ fehlen vollständig. Natrium und Kalium qualitativ erkennbar. Die freie (in Spuren vorhandene) und die gebundene Kohlensäure $\left(\mathrm{C}_{2}\right)$ wurden dem Gewichte nach nicht ermittelt, da hierzu größere Wassermengen erforderlich wären.

\section{Der Untere Sonntagskarsee.}

(Besuchsdatum: 17. August 1919.)

Höh e über dem Me eress piegel: ca. $1930 \mathrm{~m}$.

L a g e und Umgebung: Von der Unmittelbarkeit der umgebenden Gipfelreihe (Karspitzen und Vorberge des Waldhornes) wesentlich weniger betroffen als der Obere Sonntagskarsee und in einer um ungefähr $100 \mathrm{~m}$ tieferen Stufe talauswärts gelegen, befindet sich im nämlichen Gebiete des Glimmerschiefers der Untere Sonntagskarsee. 
Seine Ufer werden mit Ausnahme einer kleinen Geröllhalde im Westen und der Felsabbrüche des an der Südseite hinziehenden Riegels von begrasten Hängen gebildet. Der Bogen von Nordwesten bis nach Nordosten hat flache Uferränder, die an letzter Ste'le einer kleinen Bucht Raum geben. Als Hauptzufluß kommt der aus dem oberen See abströmende, über die Felsabbrüche des Südufers als Wasserfall fließende Bach in Betracht; außer ihm wurden noch je zwei kleinere Rinnsale im Südwestufer und Ostufer als Zuflüsse beobachtet; sie nehmen ihren Ursprung in den noch mit Schnee bedeckten Karen. Uferrand und Seefläche selbst waren vollständig schnee- bezw. eisfrei.

Gr öße: Der längste Durchmesser mißt ungefähr 300 Schritte.

Beschaffenheit des Seebodens. Warserflora: Die Bodenverhältnisse gleichen so ziemlich jenen des Oberen Sonntagskarsees; größere Tiefen finden sich zweifellos im südlichen Abschnitt. während der Grund gegen die nördliche Hälfte des Sees gleichmäßig ansteigt und eine Wasserhöhe von wenigen Metern aufweist. Der Seeboden ist von kleinen Steinen belegt, zwischen welchen sich feinsandige Flächen ausbreiten. Versumpfte Stellen fehlen vollständig. Die Wasserregetation ist gering entwickelt, wenn auch der Algenbestand an einzelnen Uferpartien im Gegensatze zum Oberen See sichtbar hervortritt; auffallend wirken die stellenweise zahlreich angehäuften Moosballen, welche mit den abrollenden Steinen in das Becken geraten sein dürften. Die Färbung des Seespiegels ist dunkelgrün.

Wassertemperatur: Dieselbe betrug um $12^{\mathrm{h}} 30^{\prime} \mathrm{p}$. m. bei bedecktem Himmel an der Oberfläche 7,50 $\mathrm{C}$, in nächster Nähe des $\mathrm{Zu}$ flusses gemessen; an drei weiter davon entfernten Stellen war sie $8^{\circ}$ bezw. $9^{0} \mathrm{C}$. Die Wassertemperatur des Hauptzuflusses zeigte (gleichzeitig) $6^{\circ} \mathrm{C}$.

Cladoceren- und Copepodenfauna: Wie das Vorkommen zahlreicher Fische, welche dem Oberen Sonntagskarsee vollständig fehlen, schon erwarten ließ, ergab die Untersuchung der Planktonnetzfänge eine reicher entfaltete Entomostrakenfauna, die aus folgenden Spezies besteht: Acroperus harpae BAIRD (hochgekielte Form), Alona affinis var. ornata Stingelin, Alona quadrangularis O. F. Miüller (Type), Canthocamptus cuspidatus SchMEIL, Cyclops serrulatus Fischer und Cyclops vernalis Fischer.

Chemische Zusammensetzung des Wassers: Das Wasser war klar, farb- und geruchlos, seine Reaktion neutral. Anzahl der Schwebebestandteile sehr gering. Der Verdampfungsrückstand von $1 \mathrm{l}$ Wasser hatte das Gewicht von 22,6 mg. Glührückstand nicht be- 
stimmt. $\mathrm{Si} \mathrm{O}_{2} 3,4 \mathrm{mg}, \mathrm{Al}_{2} \mathrm{O}_{3}+\mathrm{Fe}_{2} \mathrm{O}_{3} 2,6 \mathrm{mg}$, C'a O $11,4 \mathrm{mg}, \mathrm{Mg} \mathrm{O}$ $0,507 \mathrm{mg}$; Natrium und Kalium wurden qualitativ nachgewiesen. $\mathrm{S} \mathrm{O}_{4}$ in Spuren; $\mathrm{Cl}, \mathrm{NO}_{3}, \mathrm{NO}_{2}$ vollständig fehlend. Freie $\mathrm{CO}_{2}$ in Spuren vorhanden, dem Gewichte nach ebenso wie die gebundene Kohlensäure jedoch nicht bestimmbar.

\section{Der Mittlere Klippensee. (Textfig. 8.)}

(Besuchsdatum: 18. August 1919.)

Höhe über dem Meeresspiegel: $2257 \mathrm{~m}$.

$\mathrm{L}$ a g e u nd U m g e b u n g: Die Schladminger Tauern beherbergen in östlich gerichteter Fortsetzung des Waldhorngipfels eine „Platte“ von etwa 30 , im sogenannten Klafferkessel nahe vereinigte Wasserbecken der verschiedensten Größe und Gestalt, deren hohe Lage (2096-2431 m ü. d. M.) dem Ziele unserer Untersuchungen in ausgezeichneter Weise entspricht. Die genaue Topographie dieser seenreichen Hochgebirgslandschaft kann aus der eingehenden und vorzüglichen Darstellung von H. WöDc [„Der Klafferkessel in den Schladminger Tauern“, Zeitschrift d. D.Ö. A.V., vol. 49, 1918, p. 101-124] entnommen werden. Wir beschränken uns einstweilen auf die Mitteilung unserer Beobachtungen an dem von $H$. WöDL als mittleren Klippensee bezeichneten Gewässer. Seine Umrahmung wird durchwegs von niedrigen Felsriegeln gebildet, so daß die gesamte flache Bodenmulde einer vollständigen Besonnung ausgesetzt ist. Trotzdem lagerten zur Besuchszeit noch ausgedehnte Schneefelder am Nordost-, Südost- und Südufer des Sees und war auch der Spiegel selbst längs weiter Uferstrecken mit Eis bedeckt, während große abgeschmolzene Eistrümmer als Schollen umherschwammen. Vom unteren Klippensee wird der mittlere nur durch eine schmale, ganz niedere Bodenwelle abgetrennt, welche zugleich das Westufer bildet; ein kurzes, enges Rinnsal vermittelt die Verbindung. Als Zuflüsse sind drei kleine Bäche nachweisbar, von denen zwei dem Ostufer, einer dem Nordzipfel des Sees, unterhalb der „Unteren Klafferscharte“, angehören. Das Gestein des Klafferkessels und seiner Gipfelerhebungen besteht aus. Quarzit- (Glimmer-) Schiefer; der ganze Kessel mit seinen Seen geht zweifellos auf die Tätigkeit eines Gletschers zurück, dessen letzter Rest in einem kleinen Eisfelde im Rauhenbergkar erhalten ist.

Gr öß e: Der größte Durchmesser des rundlich geformten Wasserbeckens mißt kaum 100 Schritte.

Beschaffenheit des Seebodens. Wasserflara: Die Absenkung des Seebodens gegen die Mitte des Beckens, wo sich eine 
kleine schmale Inselbank emporhebt, ist äußerst schwach und ziemlich gleichmäßig geneigt, weshalb große Seichtwasserflächen entstehen. Die Maximaltiefe des Sees dürfte 3-4 $\mathrm{m}$ nicht übersteigen. Der Grund ist mit mehr oder weniger großen Platten des Schiefergesteines belegt, zwischen welchen er überall sandartige Beschaffenheit zeigt; versumpfte oder schlammig-lehmige Stellen fehlen vollständig. Mit Ausnahme eines recht spärlichen, dunkelgefärbten Algenbelages an den Steinen kann von einer makroskopischen Wasservegetation nichts bemerkt werden; hingegen ergab die Untersuchung der Planktonnetzfänge das Vorkommen von Planktonalgen. Die Wasserfärbung ist dunkelgrün.

W a ssertemperatur: An der Oberfläche wurden $3-5^{0} \mathrm{C}$ gemessen (bei gleichzeitiger Lufttemperatur von $10,2^{\circ} \mathrm{C}$ im Schatten um $10^{\mathbf{h}} 15^{\prime}$ a. m.); die Wassertemperatur der drei Zuflüsse betrug $5,2^{0}$, $7^{\circ}$ und $8^{\circ} \mathrm{C}$, war somit in allen Fällen höher als jene des Oberflächenseewassers selbst; die Wirkung der Besonnung auf die über das Gestein rieselnden Zuflüsse wurde im See durch das vorhandene Eis wieder abgeschwächt. (Vergl. dazu die Topograph. Skizze; Zuflüsse 1., 2. und 3.)

Clad o ceren- und Copepodenfauna: In den Proben, deren mineralogischer Detritus stets glimmerige Beschaffenheit besaß, dominierte die Cladocere Chydorus sphaericus O. F. Müller; ferner waren die Copepoden Cyclops serrulatus Fischer und Canthocamptus schmeili MrazeK in mehreren Exemplaren vorhanden, neben diesen einige Acroperus harpae BAIRD und ein 우 von Cyclops vernalis FisCHER.

Chemisehe Zusammensetzung des Wassers: Das Wasser war klar, farb- und geruchlos; seine Schwebebestandteile sehr gering; Reaktion neutral. Der Verdampfungsrückstand (1 l) betrug 9,2 mg. Si $\mathrm{O}_{2} 2,8 \mathrm{mg}, \mathrm{Al}_{2} \mathrm{O}_{3}+\mathrm{Fe}_{2} \mathrm{O}_{3} 2 \mathrm{mg}, \mathrm{CaO} 2,8 \mathrm{mg}, \mathrm{Mg} \mathrm{O} 0,5 \mathrm{mg}$; Natrium und Kalium wurden qualitativ nachgewiesen. $\mathrm{S}_{4}$ in Spuren, $\mathrm{Cl}$ in Spuren; $\mathrm{N} \mathrm{O}_{3}$ und $\mathrm{N} \mathrm{O}_{2}$ fehlten gänzlich. $\mathrm{C} \mathrm{O}_{2}$ (in Spuren vorhanden) war ebenso wie die gebundene Kohlensäure und der Glührückstand mit Rücksicht auf das geringe Wasserquantum nicht bestimmbar.

\section{Der Weißsee am Tauernjoch.}

(Besuchsdatum: 24. August 1920.)

Höhe über dem Meeresspiegel: $2218 \mathrm{~m}$.

$\mathrm{L}$ a g e und Umg e bu ng: Einer der rechtsuferigen Seitenbäche der Salzach, die Stubach, führt talaufwärts in das als Naturschutzpark in Aussicht genommene Gebiet aușgedehnter Hochwälder, dessen Gebirgshintergrund das nordwestliche, zu Salzburg gehörige Ende des mächtigen 
Großglocknerstockes mit seinen vorgeschobenen Gipfeln der: Hohen Riffl und der Granatspitzen bildet. An der Senke des Kalsertauernjoches zwischen den genannten Erhcbungen liegt der Weißsee, nächst der Rudolfshiüte des D.-Ö. Alpenvercines. Úber die Topographie dieses Beckens unterrichtet E. FugGer [1911, Mitteilg. Gesellsch. Salzburg. Landeskunde, vol. 51, p. 34, Taf. 52]. Das Gestcin der Umgebung besteht aus Granitgneis. Dem Nordostufer, wo nahezu senkrecht aufsteigende Felswände den Wasserspiegel umrenden und aus deren Fortsetzung nach abwärts die auffallend große Titfe cileses Hocl gєbirgssees resultiert, schließen sich gegen Südwesten Scluttgel änge und endlich weniger steil geneigte, sancig-schotterige Böden an; flacker, moosbewacksener Felsgrund findet sich nur am Süc.ostufer. Neben єiner größeren Anzahl kleinerer (Schmelzwasser-) Zuflüsse münden єin stärkerer Bach im Osten und ein Wasserfall im Nordwesten in den See; am Nordostzipfel entströmt der Abfluß (Weißenbacl).

Größ e: Nach den Angaben Fugaer's mißt der See in der Südwest-Nordostrichtung $540 \mathrm{~m}$ Länge, stine größte Breite beträgt $420 \mathrm{~m}$.

Beschaffenheit des. Seebodens. Wasserflora: Der Weißsee gekört zu den Thichterseen, welcke gegen das Zentrum steil abfallen; ảie Maximalticfe beträgt lier $53 \mathrm{~m}$ und nimmt eine ungefähr in der Längsrichtung des Bcckens gelegene, gestreckte Fläche ein. Soweit der Seegrund sicl tbar ist, bedecken il n Felsplatten und Blöcke, Schotter und Sand. Eine allgemeine Tiübring des Seewassers durch feinen Sand verrät sich in der milclig-gr ünlichen Farbe. Die Wasservegetation erscheint gänzlich verkümmert; es fehlt selbst der in fast allen Hocl gebirgsseen vorkommende Algeribelag an den Steinen.

Wassertemperatur: Dieselbe betrug bei vollstänciger Besonnung an der Oberfläche $5-5,1^{\circ} \mathrm{C}$. Für einen der kleinen Zuflüsse wurde von uns eine Temperatur von $4^{\circ} \mathrm{C}$ gemessen. [Die Lufttemperatur betrug zur selben Beobacktungszeit $\left(9-11^{\mathrm{h}}\right.$ a. m.) $3-5^{0} \mathrm{C}$ im Schatten.]

$\mathrm{Clad}$ o cer e $\mathrm{n}-\mathrm{u} \mathrm{n}$ d C o p e p o d e $\mathrm{n}$ f a u n a: Den beobachteten „öden“ Vegetationsverłältnissen entsprechend ergab auch die Untersuchung der Planktonnetzfänge große Artenarmut; Cladoceren felilten überhaupt, an Copepoden waren Canthocamptus cuspidatus ScHMEIL, Cyclops serrulatus Fischer und $C$. vernalis Fischer vertreten. Der See entlält keine Fiscl. $€$ !] Wenn arch der Mangel des Nackweises an Cladoceren sich späterhin nicht bestätigen sollte, so spricht er doch mit Rücksickt auf den Vergleich mit den Erg€bnissen, welche unsere Untersuckungen unter Anwendung der nämlichen Fangmethoden bei einer 
großen Zahl von Hochgebirgsseen geliefert haben, für die geringe Entfaltung der Crustaceenfauna in diesem Wasserbecken.

Chemische Beschaffenheit des Wassers: Das Wasser der Probe war milchig und enthielt zahlreiche weiße Flocken; sein Geruch stark käseartig, seine Reaktion schwach sauer; Färbung schwach gelblich. Verdampfungsrückstand (1 l) $24,2 \mathrm{mg}$, Glühverlust $11,2 \mathrm{mg}$, Glührückstand $13 \mathrm{mg}$. Si $\mathrm{O}_{2} 5,4 \mathrm{mg}, \mathrm{Al}_{2} \mathrm{O}_{3}+\mathrm{Fe}_{2} \mathrm{O}_{3} 3,11 \mathrm{mg}$, Ca $\mathrm{O} 2,03 \mathrm{mg}, \mathrm{Mg} O 2,52 \mathrm{mg}$; Natrium und Kalium wurden qualitativ nachgewiesen. $\mathrm{S} \mathrm{O}_{4}$ und $\mathrm{Cl}$ in Spuren vorhanden. $\mathrm{N} \mathrm{O}_{2}, \mathrm{~N} \mathrm{O}_{3}$ fehlen. Freie Kohlensäure vorhanden, jedoch nicht bestimmbar.

\section{Der Klammjochsee.}

(Besuchsdatum : 8. August 1921.)

Höh e über dem Meeresspiegel: $2350 \mathrm{~m}$.

Lage und Umg e bung: Der Bach des Wattentales, welcher bei dem gleichnamigen Orte (Wattens) im Unterinntale das rechte Innufer erreicht, sammelt seine Gewässer im ausgedehnten Gebiete der Tuxer Tonschiefer, die den Zillertaler Gneis- und $\mathrm{Pl}_{\text {yllitmassen }}$ bis zum rechtsseitigen Innufer vorgelagert sind. Den hintersten Talabschluß bilden die wegen ihrer komplizierten und eigenartig verworrenen geologischen Verhältnisse sehr bekannten Gipfelreihen der Tarntaler Köpfe ${ }^{1}$ ); ihr Zug bricht im Nordwesten mit der Klammspitze ab, an deren Fuße das Klammjoch einen Übergang in das Tal von Navis herstellt. Hier liegt der kleine Klammjochsee, ein ausgesprochener Seichtsee in flacher Bodenmulde, von niederen, grasbewachsenen Alpenbodenwellen unmittelbar umrahmt; von Osten nach Westen schließen sich diesem Ufer Geröll- und Felsblockfelder nach aufwärts an. Das Bett liegt im Kontaktgebiet von Kalkgesteinen (Moränen- und Gehängeschutt) und Tonschiefer. Längs des Uferrandes tritt die jeweilige Höhe des Wasserstandes je nach dem Grad der Austrocknung deutlich hervor. Zu- und Abflüsse wurden nicht beobachtet.

Gr ö B e: Das Becken mißt ungefähr $100 \mathrm{~m}$ an Länge und $60 \mathrm{~m}$ an Breite.

Beschaffenheit des Seegrundes. Wasserflora: Das Gewässer zeigt durchwegs schlammige Bodenbeschaffenheit; steiniger Grund scheint vollkommen zu fehlen. Die größte Tiefe kann im Höchstfalle

1) Vergl. hiezu E. Hartmann 1913 „Der Schuppenbau der Tarntaler Berge am Westende der Hohen Tauern". I. u. II. Teil (Schluß); Jahrb. Geolog. Reichsanstalt Wien, vol. 63 p. 207, 243 mit geolog. Karte.

A rehiv f. Hydrobiologie. Suppl.-Bd. III. 
wenige Meter betragen. Der See wird vom weidenden Vieh zweifellos als Trinkstelle aufgesucht. Die Entfaltung der Wasserflora, soweit sie makroskopisch wahrgenommen werden kann, ist gering; außer einem schwachen Belag von Algen konnte in den Uferzonen nichts nachgewiesen werden, höhere Wasserpflanzen fehlen überhaupt.

Wassertemperatur: Dieselbe betrug um $6^{\text {h }}$ p. m. (nach einem Tage vollster Besonnung) 17, $6^{\circ} \mathrm{C}$ (bei gleichzeitiger Lufttemperatur von $15,5^{\circ} \mathrm{C}$ ); der Befund beweist die starke Insolationswirkung, welche in dieser Weise nur bei einem Flachsee zum Ausdruck kommen kann.

$\mathrm{Cl}$ a d o cere $\mathrm{n}$ - u n d C o p e p o d e n f a u n a: Die entnommenen Proben enthielten als weitaus dominierenden Bestandteil Daphniden, die sich als Zugehörige der Form Daphnia longispina var. longispina forma rosea-cavifrons G. O. SARS erwiesen; ferner wurden die Cladoceren Alonella excisa Fischer und Chydorus sphaericus O. F. Müulere nachgewiesen. Von Copepoden.war Cyclops strenuus Fischer vertreten, welcher im Leben rot gefärbt war.

[Chemisehe Beschaffenheit des Wassers: Nicht untersucht.]

\section{Der Untere Torjochsee.}

(Besuchsdatum: 9. August 1921.)

Höh e über dem Mi e resspiegel: ungefähr $2200 \mathrm{~m}$.

L a g e und Umge bung: Der Tuxer Bach (Zillertal) entsendet einen Seitenast, dessen Tal als das „nasse Tux" bezeichnet wird, an den Fuß der Torwandspitze, wo eine engere sattelartige Senkung, das Torjoch, in der Richtung West-Ost nach dem Abschluß des vorhin erwähnten Wattentales hinüberleitet. Südọstlich und etwa $200 \mathrm{~m}$ tiefer als die eigentliche Paßstelle liegen der Obere und Untere Torjochsee, nur durch eine kurze Bodenstufe voneinander geschieden. Der geologische Charakter der Umgebung wird vorherrschend durch Kalkgesteine bestimmt; in Kontakt dazu tritt das dem Hauptzuge der Zentralalpen eigene „Urgestein“. Das Becken des Unteren Torjochsees selbst wird unmittelbar von niedrigen, grasbewachsenen Bodenriegeln umsäumt und besitzt insbesondere längs der Südostseite flachen Uferrand. Als Zufluß kommt vor allem der im Nordosten einmündende Ausflußbach aus dem Oberen See in Betracht; südlich von ihm wurde von uns ein kurzes Rinnsal (Quelle) als zweiter Zufluß aufgefunden. Zwischen den beiden Einmündungsstellen dieser Wasserläufe hat sich cine größere Sandbank gebildet. Der See ist während der Vormittags- 
stunden voller Besonnung ausgesetzt, dürfte jedoch später durch die im Südwest aufsteigende "Felswand der Torwandspitze beschattet werden.

Beschaffenheit des Seebodens. Wasserflora: Die Gestalt des Wasserbeckens im allgemeinen wie auch die Neigung der Seichtwasserzone, welche durchschnittlich recht schmal ist, läßt darauf schließen, daß es sich um einen tieferen, trichterartigen See handelt, wenn auch die Maximaltiefe keine auffallend große Meterzahl erreichen wird. Der Seegrund zeigt, soweit er vom Ufer aus sichtbar wird, vorwiegend schlammige Beschaffenheit. Die makroskopische Wasservegetation besteht nicht nur aus Algen, sondern auch aus höheren Wasserpflanzen, die sich insbesondere nächst der Sandbank an den Mündungsstellen der Zuflüsse stark entwickelt hat.

W a s s e r t e m per a t u r: Dieselbe betrug in der Zeit von $9-11^{\mathrm{h}}$ a. $\mathrm{m}$. an der Oberfläche $14,3^{\circ} \mathrm{C}$ (bei gleichzeitiger Lufttemperatur von $14,5^{\circ} \mathrm{C}$ im Schatten); als Temperatur des Zuflußbaches vom Oberen See wurden $12,8^{\circ} \mathrm{C}$, am Quellrinnsale $7,3^{\circ} \mathrm{C}$ gemessen.

Cladoceren- und Copepodenfauna ${ }^{1}$ : Die beiden Crustaceengruppen waren in den von uns gesammelten Planktonnetzfängen durch je zwei Spezies vertreten, nämlich durch Daphnia longispina var. longispina s. str. (КегLнаск), Chydorus sphaericus O. F. Müller und Cyclops strenuus Fischer, Diaptomus bacillifer KoELBEL.

Chemische Beschaffenheit des Wassers: Nicht untersucht.

\section{Anhang.}

Außer diesen 10 Hochgebirgsseen, deren Untersuchung unmittelbar in unseren Arbeitsplan fiel, sollen hier noch zwei Ostalpengewässer besprochen werden, die niedereren Höhengürteln angehören und innerhalb der Waldgrenze gelegen sind. Das eine von ihnen, der Grünsee im Stubachtale, war bisher bezüglich seiner Crustaceenfauna nicht untersucht, das zweite, der Krummsee im Unterinntalgebiete, lieferte einige neue Funde, die bemerkenswert erscheinen; von beiden Seen liegt nun auch die chemische Wasseranalyse vor. Die Ergebnisse werden beim Vergleich mit den gleichsinnig ausgeführten Untersuchungen an den echten Hochgebirgsseen einigen Wert besitzen.

1) Der See enthält nach unseren Beobachtungen keine Fische. - Die Farbe des Gewässers ist intensiv grün, das Wasser sehr klar und transparent. 


\section{Der Grünsee.}

(Besuchsdatum: 22. August 1920.)

Höhe über dem Meeresspiegel: $1699 \mathrm{~m}$.

$\mathrm{L}$ a g e u n d Umge bu ng: Dieser mit mehreren anderen stehenden Gewässern des Alpenbereichs gleichnamige See gehört gleich dem unter Nr. 8 besprochenen Weißsee dem Stubachtale an, liegt somit im salzburgischen Teil der Zugänge zum GroBglockner-Hauptstock. Seine Topographie wurde von E. Fugger [1899, Mittlg. d. Gesellsch. Salzb. Landeskunde, vol. 39, p. 6 Taf. 30] gründlich behandelt. Das Gestein der Seemulde besteht aus Gneis. Das Ostufer wird von einem waldigen Hang eingenommen, der gegen den Wasserspiegel mit einer felsigen Steilstufe (Gemäuer) abbricht; ebenso steht sein Nordrand und etwa das erste Drittel des Westufers an festes Felsgestein (Felswand und Gemäuer) an, während sich die übrigen zwei Drittel des letzteren aus Schuttkegelmassen zusammensetzen, die ihr Geröll dem Seegrund selbst weitergeben. Nur das Südufer des Sees wird von flachen, stark versumpften Mooswiesen gebildet, zwischen welchen der Weißenbach sein Zuflußwasser führt. Am Schnittpunkt des Nord- und Westufers mündet der Abfluß aus der sogenannten "schwarzen Lacke" in den See. Neben diesen zwei Hauptzuflüsșen sind noch zwei kleinere, als Wasserfälle zuströmende Rinnsale an der Westseite des Wasserbeckens zu beobachten.

Gr ö Be: Der Grünsee ist $420 \mathrm{~m}$ lang und $315 \mathrm{~m}$ breit.

Beschaffenheit des Seebodens. Wasserflora: Die Ränder des Sees zeigen rundum grobsteinige (plattige) Beschaffenheit, die gegen die Seemitte zu in Sand übergeht. Hievon ist nur das versumpfte Südufer ausgenommen; von hier aus senkt sich auch der Grund in geringerer Neigung gegen die Mitte, als von der West-, Nordund Ostseite. Nach den Messungen FugGer's beträgt die Maximaltiefe $32,2 \mathrm{~m}$. Der See ist außerordentlich algenreich; an allen Uferrändern sind dunkelgrün und hellgrün gefärbte Algenbestände zu beobachten. Als Zeit des Einfrierens wird der Beginn des Dezember, als Tauzeit Mitte Mai angegeben. In der Süd-Nordrichtung besteht eine deutlich wahrnehmbare Wasserströmung.

Wassertemperatur: An der Oberfläche betrug dieselbe (um $10^{\mathbf{h}} 15^{\prime}$ a. m. des Besuchsdatums) 7,80 - $8^{0} \mathrm{C}$; als Zuflußtemperatur des Weißenbaches wurden von uns gleichzeitig $6,3^{\circ} \mathrm{C}$ gemessen.

Cladoceren- und Copepodenfauna: Die Planktonnetzfänge, die an diesem See von einem Boote aus ausgeführt werden 
konnten und von der Oberfläche bis aus einer Tiefe von $10 \mathrm{~m}$ stammen, enthielten (neben dem quantitativ reich vertretenen Phytoplankton) merkwürdigerweise keine Copepoden, ausgenommen ein vereinzeltes Cyclops-Nännchen; an Cladoceren waren vertreten Acroperus harpae BaIrd, Alona affinis (Leydig) und Chydorus sphaericus O. F. Müller. Es fehlten somit auch typisch eulimnetische Formen dieser zweiten Crustaceengruppe. Als Ursache dieser Erscheinung kann vielleicht die immerhin stark ausgeprägte Wasserströmung angesprochen werden. Leider verhinderten durchaus ungünstige Witterungsverhältnisse eine weitere Fortsetzung unserer Aufsammlungen, so daß die Lücke der Speziesliste nicht einwandfrei gellärt ist. (Der See ist forellenreich.)

Chemisehe Zusammensetzung des Wassers: Die Wasserprobe zeigt schwach gelbliche Färbung, enthielt als Schwebebestandteile zahlreiche weiße und schwarze Flocken, war jedoch geruchlos und hatte neutrale Reaktion. Verdampfungsrückstand (1 l) $37,2 \mathrm{mg}$; Glühverlust 8,2 mg, Glührückstand $29 \mathrm{mg}$. $\mathrm{Si}_{2} 4,6 \mathrm{mg}, \mathrm{Al}_{2} \mathrm{O}_{3}+\mathrm{Fe}_{2} \mathrm{O}_{3}$ 6,6 mg, Ca $\mathrm{O} 14 \mathrm{mg}, \mathrm{Mg} O 3,6 \mathrm{mg}$; Kalium qualitativ nachweisbar. $\mathrm{Cl}$ und $\mathrm{S} \mathrm{O}_{4}$ in Spuren vorhanden, ebenso freie Kohlensäure $\left(\mathrm{C} \mathrm{O}_{2}\right) . \mathrm{H} \mathrm{N} \mathrm{O}_{3}$ und $\mathrm{H} \mathrm{N} \mathrm{O}_{2}$ fehlen vollständig.

\section{Der Krummsee. (Textfig. 13.)}

(Besuchsdatum: 20. Juli 1922 und 4. Oktober 1922.)

Höh e über dem Mieeresspiegel: $557 \mathrm{~m}$.

L a g e u n d U m g e b u n g: Das Wasserbecken, welches zur Gruppe der Reintaler Seen gezählt wird, liegt im waldreichen Mittelgebirge bei Kramsach im Unterinntale Tirols. Nach BlaAs [1902] setzen sich die Böden der ganzen Gegend, in welcher auch die Miulde des Krummsees gelegen ist, aus Glazialschutt zusammen, welcher über Dolomit und Tertiärschichtengesteinen lagert. Die unmittelbare Umrandung des Beckens besteht vorwiegend aus hügeligem Gelände mit Wiesengrund; zwei solche Hügel des Ostufers springen nebeneinander als Landzungen weit vor und geben hiedurch der Seefläche jene eigenartig gelappte Gestalt, von welcher vermutlich die Benennung des Gewässers stammen dürfte. Mit Ausnahme einẹ Strecke der Südseite, wo sich die Bootsund Badehütten befinden, zeigen sich fast rundum Schilf- und Seerosenbestände, ganz besonders verschilft ist ein großer Teil des Nordwestrandes und die nördliche, s-förmig gewundene Hälfte des Sees, in welchen 
am ostwärts gerichteten Endzipfel der Zuflußbach aus dem eigentlichen Reintaler See einmündet. Eine topographische Aufnahme des Wasserbeckens stammt von J. Müllner [1905].

Grö Be: Der Flächeninhalt des Krummsees beträgt 4,9 Hektar; Maximallänge $350 \mathrm{~m}$, größte Breite $150 \mathrm{~m}$.

Beschaffenheit des Seebodens. Wasserflora: Der Krummsee stellt einen Seichtsee dar, dessen Maximaltiefe von 2,5 m eine langgestreckte, inselartige Fläche in der Längsrichtung der südlichen Beckenhälfte einnimmt. Die Bodenbeschaffenheit ist außerordentlich schlammig, am Uferrand nur auf kleineren Strecken feinsandig. Die Entfaltung höherer Wasserpflanzen (Schilf, Seerose, Tausendblatt) und Entwicklung zahlreicher makroskopischer Algen im Verein mit reichem Phytoplankton charakterisiert den See als pflanzenreiches Gewässer. [Es sei hier erwähnt, daß der Krummsee zahlreiche Fische und die Teichmuschel enthält; nach Aussage des Besitzers kommen an ersteren vor: Lauge (Squalius), Pirschling (Perca), Weißfisch (Alburnus), Hecht (Esox) und Karpfen (Cyprinus), der letzte eingesetzt.]

W a s serte mper a tur: Nach Brenm [1907], welcher den See (vermutlich im August) auf seinen qualitativen Planktongehalt untersuchte, erreicht das Wasser Sommertemperaturen bis zu $23^{\circ} \mathrm{C}$. Von uns wurde am 20. Juli eine Oberflächenwassertemperatur von $17,25^{\circ} \mathrm{C}$ (bei gleichzeitiger Lufttemperatur von $13,25^{\circ} \mathrm{C}$ zur Zeit von $10-11^{\mathbf{h}}$ a. m.), am 4 . Oktober eine ebensolche von $13,5^{0} \mathrm{C}$ (zur Zeit von $2-2^{\mathrm{h}} 15^{\mathrm{\prime}}$ p. m.) abgelesen.

Chemische Beschaffenheit des Wassers: Die Probe (1 1 Oberflächenwasser) ist klar und durchsichtig; beim Schütteln zeigen sich graue Flocken; Geruch (mäßig) nach $\mathrm{N} \mathrm{H}_{3}$ und $\mathrm{S} \mathrm{H}_{2}$; Reaktion alkalisch. Verdampfungsrückstand 245,0 mg; Glühverlust 91,4 mg; Glührückstand $153,6 \mathrm{mg}$. $\mathrm{Si}_{2} 5,26 \mathrm{mg}, \mathrm{Al}_{2} \mathrm{O}_{3}+\mathrm{Fe}_{2} \mathrm{O}_{3} 12,86 \mathrm{mg}$, $\mathrm{Ca} O$ 76,26 mg, $\mathrm{Mg} O$ 54,78 mg; Kalium und gebundene $\mathrm{C}_{2}$ in Spuren, ebenso Cl, $\mathrm{H}_{2} \mathrm{~S} \mathrm{O}_{4}$ und $\mathrm{N}_{2} \mathrm{O}_{5}$ in Spuren vorhanden. [Die Gewichtsmengen von $\mathrm{CaO}$ und $\mathrm{MgO}$ entsprechen 14 deutschen Härtegraden!]

Clad oceren- und Copepodenfauna: Über die Copepoden- und Cladocerenfauna des Krummsees liegen bereits Angaben von BREнм [1907] vor; da dieselben mit den Ergebnissen unserer eigenen Planktonnetzaufsammlungen, welche vom 20. Juli, 15. August und 4. Oktober 1922 stammen, in wichtigen Punkten nicht übereinstimmen, so seien hier die beiden Listen nebeneinandergestellt: 


\section{BREHM [1907].}

Cyclops sp. jur.

Cyclops macrurus.

Ceriodaphnia pulchella.

Diaphanosoma brachyurum.

Graptoleberis testudinaria.

Peracantha truncata.

Scapholeberismucronata (fronte cornuta).

Sida crystallina.

\section{Pesta 1922.}

Cyclops sp. jur.

Cyolops leuckarti.

Cyclops macrurus.

Diaptomus gracilis.

Acroperus harpae.

Alona guttata (f. typica + tuberculata).

Bosmina longirostris (f. typica + cornuta).

Ceriodaphnia pulchella.

Diaphanosoma brachyurum.

Graptoleberis testudinaria.

Peracantha truncata.

Sida crystallina.

Vom Nachweise der beiden Cladoceren Acroperus und Alona abgesehen, wurden demnach von mir nicht weniger als 3 Planktonvertreter (Cyclops leuckarti, Diaptomus gracilis, Bosmina longirostris) angetroffen, welche BReHM nicht erwähnt; dieselben waren in allen Proben in außerordentlich großen Mengen vorhanden, so daß es als ganz ausgeschlossen gelten kann, daß sie etwa seinerzeit von BREHм hätten übersehen werden können. Hingegen wurde von mir kein einziges Exemplar der von BREHM aufgezählten Scapholeberis mucronata beobachtet. F ür d e n $\mathrm{m}$ e r kwürdigen Befund bleibt kaum eine andere Erklärung als die Annahme einer natürlichen Veränderung inder Zusammensetzung des Planktons. Scapholeberis scheint durch Bosmina verdrängt, Cyclops leuckarti und Diaptomus gracilis fanden als neue Elemente in dem pflanzenreichen nnd warmtemperierten See von ausgesprochen eutrophem Typus ejn Optimum ihrer Existenzbedingungen. Die Beobachtung dieser Faunenänderung ist geeignet, unsere Aufmerksamkeit auf eine Reihe von Fällen zu lenken, die aus dem 4. Abschnitte dieser "Studien“ zu entnehmen sind; einander widersprechende Angaben über das Vorkommen bezw. Fehlen einzelner Gattungen und Arten in einem und demselben Wasserbecken durch verschiedene Autoren gaben schon mehrmals Anlaß zu mehr oder weniger kritikvollen Bemerkungen (vgl. z. B. Nr. 1 Achensec bezüglich Diaptomus, Nr. 22 Caldonazzosee bezüglich Bosmina, Nr. 29 Faakersee bezüglich Leptodora, Nr. 40 Gardasee bezüglich Sida, Nr. 51 
Hintersteiner See bezüglich Copepoda, Nr. 65 Königssee bezüglich Diaptomus bacillifer u. gracilis, Nr. 123 Unterer Plenderlesee bezüglich Copepoda und Cladocera, Nr. 128 Reintaler See bezüglich Copepoda und andere Fundorte!). Dureh die Feststellung derartiger Faunenänderungen, welchesichimLebensraume eines Sees innerhalb des Ablaufes einer mehroder weniger langen Jahresreihe vollziehen w ürden, könnten der hydrobiologischen Untersuchung jn mancher Hinsicht ursächliche Zu sammenhänge aufgedeckt erscheinen, während andererseits ohne Zweifel folgt, daßdaraus der Auswertung faunistiseher Beobachtungen vermehrte Schwierigkeiten erwachsen wären.

Bei einer übersichtlichen Rückschau auf die bisher von mir selbst besuchten, oberhalb der Waldgrenze glegenen Hochgebirgsseen (20) des Ostalpenbereiches heben sich i m Ge gen sat ze zu den S e en der Waldregionen folgende Hauptmerkmale in bezug auf die allgemeinen morphologischen (topographisch-hydrographischen) Eigenheiten dieser Gewässer heraus:

1. Geringe Flächenausdehnung; bei der überwiegenden Miehrzahl echter Hochgebirgswasserbecken bewegen sich die Längen- und Breitenmaße in Grenzwerten von $500 \times 200 \mathrm{~m}$; Maße von $1000 \mathrm{~m}$ oder mehr werden nicht erreicht.

2. Steiniges (Geröllschutt oder Fels) Ufer; dieser Charakter entspricht der mehr oder weniger unmittelbaren Annäherung an die umgebenden Höhenkämme und Berggipfel.

3. Steinig-sandiger Seeboden (Seegrund); er resultiert ebenfalls aus der Uferbeschaffenheit, die auch unter Wasser ihre Fortsetzung findet.

4. Schmalste Entwicklung einer Uferbank; die Ausdehnung von Seichtwasserzonen erscheint sehr beschränkt, soweit dieselbe im Verhältnis zur Seetiefe und Wasseroberfläche steht.

5. Geringe Wassertiefe; sie liegt im allgemeinen in Grenzen zwischen 4-15 m. Während der ausgesprochene Seichtseetypus von $1 / 2-4 \mathrm{~m}$ Tiefe häufiger vorkommt, stellen erheblichere Wassertiefen $(20-50 \mathrm{~m})$ im Hochgebirge eine große Seltenheit dar. 
6. Große Klarheit des Wassers; sie fällt dem Beobachter sofort durch die weite Einsicht auf den Seegrund gegen die Seemitte zu auf; Trübungen sind auf mineralogischen Detritus zurückzuführen. [Bezüglich der chemischen „Reinheit“vergl. die Ergebnisse im 2. Abschnitt dieser "Studien".]

Allen oberhalb der Waldgrenze gelegenen Hochgebirgsseen ist ein langdauernder Eisabschluß gemeinsam; ihre "eisfreie" Zeit beschränkt sich häufig auf die Sommermonate. Das Seewasser ist nicht nur bedeutenden täglichen Temperaturschwankungen (durch die erhöhte Sonnenwirkung tagsüber und stärkere Abküh.ung über Nacht), sondern auch länger andauernden, temporären Abkühlungen auf Wintertemperaturen durch Schlechtwettereinbruch ausgesetzt. Die Tages-Oberflächєnwasser-Temperatur in den von mir während der Sommermonate Juli und August untersuchten Seen blieb in 16 Fällen unter $15^{\circ} \mathrm{C}$ und nur in 3 Fällen (Großer und Kleiner Bödensee, Klammjochsee), die sich auf extreme Seichtwasserseetypen beschränken, wurden höhere Werte $\left(16,2^{0}, 17,6^{\circ}\right.$ und $18,7^{\circ} \mathrm{C}$ ) abgelesen. Als meistvertretene Temperatur des Oberflächenwassers sind $5-10^{\circ} \mathrm{C}$ anzugeben.

Gegenüber den Gebirgsseen der Waldregionen weisen die Hochgebirgsseen folgende biologische Besonderheiten auf:

1. Sehr spärliche Entwicklung einer makroskopisch wahrnehmbaren Wasservegetation; dieselbe beschränkt sich in der überwiegenden Mehrzahl der Wasserbecken auf Algen, während litorale Vegetationsbestände (Potamogeton, Chara, Schilf u. dgl.) höherer Wasserpflanzen nur sehr selten und vornehmlich bei ausgesprochenen Seichtwasserseen (dann meist auch mit teilweise sumpfigem Uferrand) auftreten. [Vergl. dazu die Endergebnisse im 2. Abschnitt dieser „Studien“.]

2. Eine artenarme Copepoden- und Cladocerenfauna. [Verg]. dazu die anschließenden Angaben und die Spezieslisten von Seen der Waldregionen im 4. Abschnitte dieser "Studien“.]

Für den echten Hochgebirgssee dürfte die Anwendung der z. B. von Naumann angewandten Ausdrücke von "Oligotrophie“ und „Eutrophie" eine wesentlich andere Deutung erfordern und soll daher nicht ohne Diskussion einfach übertragen werden.

Über die Copepoden- und Cladocerenspezies, welche in den oberhalb der Waldgrenze gelegenen Hochgebirgsseen der Ostalpen bisher beobachtet wurden, seien an dieser Stelle einige zusammenfassende Daten 
angeführt, welche sich auf die horizontale und vertikale Verbreitung im Gebiete beziehen.

Aus dem in dieser Darstellung behandelten Ostalpengebiet sind folgende 52 (oberhalb der Waldgrenze gelegene) Hochgebirgsseen in bezug auf ihre Copepoden- und Cladocerenfauna ${ }^{1}$ ) untersucht worden:

ü. d. M.

Anderlesee . . . . 2000 m

Kleiner See südlich vom

Anderlesee .... 2000,

Kleiner See westlich rom

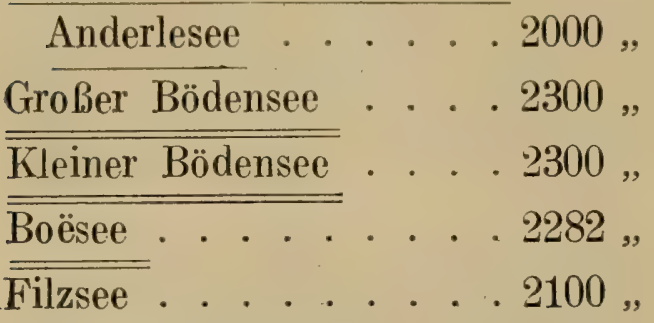

Hinterer Finstertalersee . 2259 ,

Vorderer Finstertalersee . 2235 ,

Foscagnosee ....22235,

Fraëlesee . . . . . 1934 ,

Fresenha'ssee . . . . 2150 ,

Gafiensee . . . . . 2312,

Garschinasee . . . . 2189,

Oberer Giglachsee . . . . 1960 ,

Kampspitzsee. . . . . 2200 ,

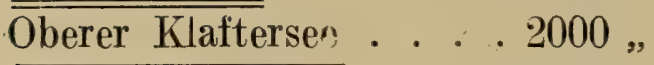

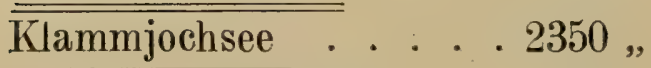

Mittlerer Klippensee . . . 2257 ,

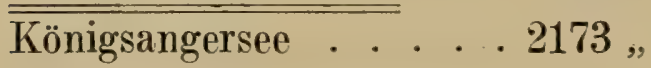

Kreuzjochsee . . . . 2200,

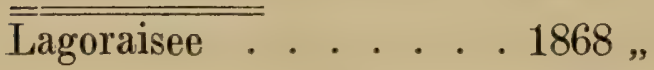

Oberer Lanischsee . . . 2300 ,

$\overline{\text { Unterer Lanischsee } ~, ~ . ~ . ~} 2200$,

Lauterersee . . . . . . 2400 , ï. d. M.

Lichtsee . . . . . . $2100 \mathrm{nt}$

Lünersee . . . . . . 1943 ,

Oberer Mühldorfersee . . 2333 ,

Unterer Mühldorfersee . . 2281,

Mutterbergersee . . . . 2483 ,

Partnunersee . . . . . 1874,

Pfitscherjochseen . . . 2284 ,

Oberer Plenderlesec . . . 2327 ,

Unterer Plenderlesee . . . 2250 ,

$\overline{\text { Unterer Pochartsee }}$. . . 1846 ,

Saualmsee. . . . . 1950,

Saureggersee . . . . 2000,

Schlickersee . . . . . 2500,

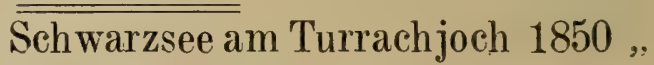

Schwarzsee im Zillertal. . 2469 ,

Unterer Seebisee . . . 2229 ,

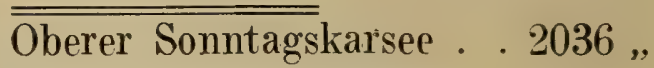

Unterer Sonntagskarsee .1930 ,

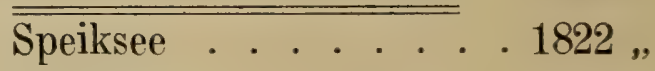

Stellunesee . . . . . . 2140,

Tilisunasee . . . . . 2102,

Todtalpsee . . . . . 2340,

Unterer Torjochsee . . 2200,

Tschampeisee . . . . 2100"

Weißsee am Tauernjoch . 2218 ,

Wildsee am Wildseeloder . 2029 ,,

Zirmsee . . . . . . 2500 ,

1) Vergl. hiezu die Charakteristik der 197 Ostalpenseen im 4. Abschnitt dieser „Studien", welche alle Wasserbecken enthält. - Die doppelt unterstrichenen Seen sind rom Verfasser selbst aufgesucht, die einfach unterstrichenen nur in bezug auf ihr faunistisches Material untersucht worden. 
$\mathrm{Zu}$ vorstehender Liste sei zunächst bemerkt, daß über die Fauna des Foscagno- und des Fraëlesees leider noch keine Angaben publiziert wurden, da die Bearbeitung noch nicht beendet ist, wie R. Mostr unsere diesbezügliche Anfrage beantwortet hat. Die Fänge aus dem Königsangersee gingen vor Abschluß der Bestimmung der Formen zugrunde. Über die Fauna des Unteren Pochartsees, welchen Brenu [1907] erwähnt, gibt der Autor aus unbekannten Gründen keinen Bericht. Der Obere Plenderlesee soll nach Breha und Zederbauer [1904] überhaupt keine tierischen Bewohner enthalten und bezüglich des Todtalpsees, welchen Zснокке [1894] untersuchte, fehlen Beobachtungen über das Vorkommen von Cladoceren und Copepoden.

Von den restlichen 46 der genannten Hochgebirgsseen gehören 33 den Zentralalpen und 13 den Kalkalpenzonen (Nördl. und Südl. Zone) an. Untersuchungen über die Fauna der in diesen letzteren Zonen gelegenen Wasserbecken sind somit besonders wünschenswert. - Es interessiert nun vorerst die Frage, welche Arten in jenen Höhen nachgewiesen wurden; eine Durchsicht der Spezieslisten aus den 46 Hochgebirgsseen des Ostalpengebietes ergibt folgende Reihe: Canthocamptus cuspidatus, echinatus var. luenensis, minutus, rhaeticus, schmeili, schmeili var. hamata, zschokkei; Cyclops albidus, fimbriatus, serrulatus, strenuus, vernalis; Diaptomus bacillifer, denticornis, gracilis, tatricus; Heterocope saliens [17 Copepoden]. Acroperus harpae; Alona affinis, quadrangularis, rectangula; Alonella excisa, nana; Bosmina coregoni, longirostris; Chydorus pigcr, sphaericus; Daphnia longispina, longispina var. hyalina, longispina var. longispina, pulex; Leydigia acanthocercoides; Macrothrix hirsuticornis, laticornis; Peracantha truncata; Polyphemus pediculus; Rhynchotalona rostrata; Simocephalus vetulus [21 Cladocera].

Eine Prüfung auf die Häufigkeit des Vorkommens dieser Spezies im allgemeinen und auf ihr Auftreten in den drei Zonen im besonderen führt zu einigen beachtenswerten Ergebnissen. Es zeigt sich, daß 5 Spezies in den Hochgebirgseen aller Zonen zahlenmäßig am stärksten vertreten, d. h. in den Faunenlisten am meisten wiederholt verzeichnet sind, nämlich: $C h y$ dlorus sphaericus (21mal), Cyclops serrulatus (21mal), Alona affinis (17mal), Cyclops vernalis (14mal) und $C y$ clops stre nnus (13mal). Diesen Arten kommen Daphnia longispina var. longispina (9mal) und Diaptomus bacillifer (8mal) noch am nächsten, wobei sich ihr Nachweis ebenfalls auf die. Kalkalpen wie Zentralalpen zugleich bezieht; wenn man berücksichtigt, daß Daphnia longispina 
ohne Hinzufügung der Varietät noch aus weiteren 5 Fundorten gemeldet ist, so dürfte es sich mit großer Wahrscheinlich keit gerade um die var. longispina handeln, so daß die angege bene Zahl zu erhöhen wäre. Gegenüber Diaptomus bacillifer ist $D$. denticornis blo $B$ in $3, D$. gracilis in 2 Seen, jedoch ebenfalls in beiden Zonen vertreten. Ganz analog verlält es sich mit dem Nachweis von Alona quadrangularis (3mal), Alonella excisa (3mal) und Daphnia pulex (3mal). Auffallend selten scheint das Genus Bosmina die oberhalb der Waldgrenze gelegenen Wasserbecken zu besiedeln; $B$. coregoni sowohl wie $B$. longirostris wurden bisher nur aus je einem einzigen Fundort der Zentralalpen bekannt. Die gleiche Seltenheit trifft für das Vorkommen von Polyphemus pediculus und Simocephalus vetulus (2 unmittelbar benachbarte Hock gebirgsseen in den Südl. Kalkalpen) zu. Über die Verteilung der Canthocamptus-Spezies läßt sich derzeit nichts aussagen, da diese Formen der Beobachtung wohl vieifach entgangen sind. Hingegen fehlt bisher der Nackweis der sicherlich nicht leicht zu übersehenden Heterocope saliens im Hochgebirge der Kalkalpenzonen, während sie in 5 Sєen der Zentralaipen angetroffen wurde; ein ganz ähnliches Verbreitungsbild liefert das Vorkommen von Acroperus harpae, welcher bereits aus 7 Hoct gebirgsseen der Zentralalpen, aber nur aus einem solchen Wasserbecken der Kalkalpenzone gemeldet ist; man darf in diesem letzten Falle, wo es sich um einen Litoralbewohner handelt, auf das Bekanntwerden weiterer Fundorte aus dem Hochgebirge sehr gespannt sein. Auch die verhältnismäßig selten beobachtete (4ma?) Rhynchotalona rostrata stammt in allen Fällen aus der Zone der ostalpinen Kalkalpen.

Alle übrigen Copepoden- und Cladocerenspezies, welche im Hochgebirge der Ostalpen beobachtet wurden, stellen bisher noch Einzelvorkommen dar; zum Teil sind sie zweifellos seltene Elemente in dieser Fauna, zum Teil dürften die Beobachtungen lückenhaft sein.

Die bisher gewonnenen Daten über das Auftreten der in den Hochgebirgsseen der Ostalpen lebenden Cladoceren und Copepoden zeigen somit, daß gewisse Arten keineswegs gleichmäßig über die zwei großen petrographischen Hauptzonender Zentral-und Kalkalpenverbreitet sind. In dieser Richtung fortgesetzte Untersuchungen werden darüber Klarheit $z u$ schaffen haben, ob und in welcher Relation der petrograph ische Bau des Gebirges als Faktor für die genannte Verbreitung maßgebend sein kann. [Vergl. hiezu die Ergebnisse des 2. und 5. Abschnittes dieser „Studien".] 


\section{A bschnitt.}

\section{Die chemische Beschaffenheit des Wassers einiger Hochgebirgsseen der Ostalpen.}

Die chemische Beschaffenheit des Wassers jener zahlreichen Secbecken der Alpen, welche allen künstlichen Eingriffen entrückt geblieben sind, ist bislang noch kaum untersucht worden. Wo es sich um höher gelegene Gewässer, also um Gtbirgsseen im engeren Sinne handelt, war zunächst auch kein Anlaß für derartige Arbeiten gegeben, da die chemische Reinheit des Wassers offenkundig vorhanden sein und allgemeine Geltung haben mußte; es fehlte somit ein Interesse seitens des Fischereibetriebes sowohl wie auch von seiten der Abwässerkunde. Zudem setzen sich solchen Untersuchungen weit erheblichere Schwierigkeiten entgegen als jenen, die an bequem zugänglichen, großen Seen der Haupttäler und Niederungen ausgeführt werden können. Daß jedoch dem Vorherrschen gewisser chemischer Bestandteile gerade in den Seebecken des Gebirges wie z. B. den Calcium- und Siliciumverbindungen eine Bedeutung für die Zusammensetzung der Wasserfauna zugesprochen werden dürfe, erscheint von vorneherein nicht unwahrscheinlich. Schon WEITH [1880, p. 102] hat sich - allerdings in alleiniger Beziehung auf den Fiscl bestand - dahin ausgesprochen, daß ,unter sonst genau gleichen Verhältnissen von verschiedenen Gewässern dasjenige das reichste an Fischen sein wird, welches die größte Menge an doppeltkohlensaurem Kalk enthält". Ähnlich äußern sich später PAvEsi [1889] und Monti [1906] im allgemeinen über die Fauna einiger Gebirgsseen. Die bestehenden Ansichten über die Einwirkung eines chemischen Faktors arf das Vorkommen und Auftreten verschiedener Süßwasserbewohner erhellen ferner zur Genüge aus den Zitaten welche sich in Steuer's Planktonkunde $[1910$, p. 27, 28, 29] finden, wo es unter anderem z. B. von der Cladocere Holopedium gibberum heißt: „es hat den Anschein, als ob dieses Tier bloß in kalkarmen Gewässern sich wohlfühle, ist es doch bei uns (in der Schweiz) wie auch anderwärts nur in Seen der Urgebirge (Vogesen, Schwarzwald, Böhmerwald, Zentralalpen, Skandinavien, Rocky Mountains) „noch nicht aber im Jura und in den Kalkalpen aufgefunden worden". Weitere Ausführungen enthalten die anschließenden Zeilen im genannten Werke. Allen diesen Gedanken liegt zweifellos die Annahme zugrunde, daß die Kenntnis der Geologie der Umgebung des betreffenden Seebeckens zur Beurteilung des Mangels oder des Vor- 
herrschens im Kalkgehalte bezw. Kieselsäuregehalte des Wassers schon genügend sei; es soll später gezeigt werden, inwieweit dies zutrifft. Nach Zsснокке [1900] liegen „faunistisch sehr arme Seen mitten in den Kalkmassen des Rhätikon, sehr reiche Becken dagegen in die Urgebirgsformationen des St. Bernhard eingesehlossen". Verschiedene Autoren haben sich somit, wie aus den eben genannten Literaturnachweisen ersichtlich ist, über den Versuch, eine gesetzmäßige Wcehselbeziehung zwischen Chemismus und Wasserfauna der natürlichen Seebecken nachzuweisen, sehr abweichend voneinander ausgesprochen und ihre Mieinung teils in mehr verallgemeinernder Form ${ }^{1}$ ), teils in Anwendung auf bcstimmte Einzelfälle niedergelegt. Um der Lösung der Frage näherzukommen, stehen zwei Wege zur Verfügung: der Weg des Experimentes (Zuchtkulturen in Lösungen) und der Weg vergleichender Beobachtung in natura (Standortsuntersuchungen). Auf die "lohnende Aufgabe “ des ersten hat bereits Steuer [1910, p. 29] aufmerksam gemacht. Er wurde in bezug auf Süßwasserentomostraken unseres Wissens zum ersten Male von Hartmann [1916] beschritten, welcher das Verhalten seines Cladocerenmateriales außerdem gleichzeitig am reien Standort verfolgte. Über den direkten Einfluß des Schwefelwasserstoffes $\left(\mathrm{H}_{2} \mathrm{~S}\right.$ ) auf die Wasserfauna, speziell auch auf Cladoceren und Copepoden, sind von BurcKHARDT [1910] sehr glücklich gewählte Beobachtungen am Ritomsee (Schweiz, Kanton Tessin, $1829 \mathrm{~m}$ ü. d. Meere) angestellt worden. [Vergl. darüber auch den „Bericht der hydrobiologischen Kommission der Schweizer Naturf.-Gesellsch." in den Verhandlungen der Schweiz. Nat.Ges. 1918, I. Teil, p. 69.] Von einschlägigen Untersuchungen außerhalb des Gebietes der Alpen haben die Arbeiten von BRönsted und WesenberG-Lund [1911] die größte Bedeutung eriangt.

Unseren eigenen Untersuchungen, die den Weg der natürlichen Standortsbeobachtung wählten und über welche nachstehend berichtet werden soll, lagen folgende Überlegungen zugrunde: Wenn es richtig ist, daß die chemische Beschaffenheit der Gewässer von Gebirgsseen in unmittelbarer Abhängigkeit von dem geologischen Bau ihrer Umgebung (Niederschlags- und Zuflußgebiet) steht, so wird die durch die Verschiedenheit in der chemischen Zusammensetzung begründete Beeinflussung auf das Vorkommen der Faunenelemente vor allem an jenen Seen einwandfrei festgestellt werden können, denen die gleichen physikalisch-klimatischen Verhältnisse zukommen; bei einer derartigen Auswahl der Standorte

1) Vergl. auch F. DAHL $[1921$, p. 19]. 
darf die störende Mitwirkung aller übrigen „Milieubedingungen“ als ausgeschlossen gelten ${ }^{1}$ ). Das Bereich der Ostalpen mit seinen drei im dritten Abschnitte dieser "Studien“ kurz skizzierten geologischen Hauptzonen erwies sich daher für die Untersuchung sehr geeignet; andererseits mußte die Wahl der Wasserbecken auf die in der waldfreien Hochgebirgsregion gelegenen Seen fallen, da hier von vornherein annähernd gleiche Existenzbedingungen im Milieu selbst zu erwarten waren.

Es mag genügen, nur mit wenigen Worten auf die Hemmnisse hinzuweisen, welche sich der Aufsammlung der Proben und den Arbeiten in jenen alpinen Höhenzonen naturgemäß entgegenstellen; wer mit dem Hochgebirge einigermaßen vertraut ist, wird dieselben ohnedem entsprechend einzuschätzen wissen. Vor allem ist der Beförderung größerer Mengen Seewasser aus naheliegenden Gründen eine Grenze gesetzt; die chemische Analyse mußte sich durchschnittlich mit einem Litermaß begnügen, was zur Folge hatte, daß einzelne Bestandteile quantitativ nicht ermittelt werden konnten (z. B. Natrium, Kalium; gebundene und halbgebundene Kohlensäure). Den Planktonnetzfängen, mittelst welcher das Cladoceren- und Copepodenmaterial gesammelt wurde, standen nur die Methoden des Uferfanges zur Verfügung, da an jenen Hochgebirgsseen Boote nicht gehalten werden, der Gedanke an die Mitnahme eines eigenen Faltbootes jedoch sofort fallen gelassen werden mußte. Bei hinreichend sorgfältigem Arbeiten und halbwegs günstigen Bodenverhältnissen birgt der Uferfang in jenen Regionen allerdings weit geringere Fehlerquellen, als man dabei in den Seen tieferer Lagen stets zu gewärtigen hat; denn im Hochgebirgsbecken gelangen auch die eulimnetischen Spezies bis an die Umrandung und steigen weniger in die Tiefe $a b$. Übrigens darf wohl bei der best- und längstdurchforschten Lokalität die gewonnene „Liste“ nicht auf absolute Vollständigkeit Anspruch erheben! - Die im Jahre 1911 begonnenen Untersuchungen [vergl. dazu Verhandlungen d. Zool. Botan. Gesellsch. Wien, vol. 52 (1912) p. 158, vol. 54 (1914) p. 210 und vol. 5̌ (1915) p. 227] bezogen sich zunächst auf die Alpen Tirols, fanden dann aber während der Jahre 1918, 1919 und 1920 ihre Fortsetzung im Gebiete des Niederen und Hohen Tauernzuges Steiermarks und Salzburgs [vergl. dazu Kapitel I dieser Studien], so daß nunmehr insgesamt 13 Analysen von Hochgebirgsseen (1930-2483 m ü. d. M.) aus dem Bereiche der Ostalpen vorliegen, denen noch je eine Analyse von dem innerhalb der Waldgrenze liegenden

1) Vergl. die diesbezüglichen allgemeinen Ausfübrungen von A. Thienemanx [1913]. 
Grünsee (1699 m ü. d. M.) und Krummsee (557 m ü. d. M.) angeschlossen wurde. Die Ergebnisse der chemischen Untersuchung ${ }^{1}$ ), welche von den Herren Prof. Hermann Klein (Wien) und Ingenieur Dr. Walther stammen, sind aus der folgenden tabellarischen Zusammenstellung zu entnehmen:

In kurzgefaßter Darstellung kann man zunächst als Ergebnis des $q$ u alitat, ive n Nachweises aus der Tabelle folgendes festhalten:

1. Salpetersäure $\left(\mathrm{H} \mathrm{N} \mathrm{O}_{3}\right)$ und salpetrige Säure $\left(\mathrm{H} \mathrm{N} \mathrm{O}_{2}\right)$ iehlen durchwegs; alle 13 untersuchten Seewässer des Hochgebirges sind diesbezüglich als "rein“" zu bezeichnen.

2. Ammoniak $\left(\mathrm{N} \mathrm{H}_{3}\right)$ mangelt in 10 Seen vollständig; nur in drei Wasserbecken wurde es in Spuren nachgewiesen. Dieses Ergebnis ist auffällig; es liegt der Gedanke nahe, daß in jenen drei Fällen eine Verunreinigung durch weidendes Vieh (insbesondere Schafe und Ziegen) stattfindet. Dem ist nun allerdings entgegenzuhalten, daß diese Möglichkeit für mindestens fünf der ammoniakfreien Wasserbecken besteht, wie aus unseren Beobachtungen an den Fundstellen hervorgeht; es würde somit dieser Mangel wiederum eine neue Aufklärung erfordern. Ein Vergleich mit den in der Rubrik über die Entfaltung der submersen Wasserflora angeführten Daten zejgt jedoch deutlich eine Korrelation, indem die drei ammoniakhältigen Seen gerade auf solche Fundorte entfallen, in welchen sich außer Algen auch eine reichere Vegetation höherer Wasserpflanzen vorfindet. Wir glauben daher das Vorkommen von Ammoniak als Produkt stärkerer vegetabiler Verwesungsprozesse ansprechen zu können.

3. Spuren von freier Schwefelsäure $\left(\mathrm{H}_{2} \mathrm{~S} \mathrm{O}_{4}\right.$; berechnet als $\left.\mathrm{SO}_{3}\right)$ sind in allen Seebecken nachgewiesen; ihre Bildung darf wohl mit ziemlicher Sicherheit der Oxydation von Schwefelwasserstoff, dem Zersetzungsresultat der in den Wasserproben enthaltenen organischen Substanzteilchen, zugeschrieben werden.

4. Ein Chlorgehalt $\left(\mathrm{Cl}^{2}\right)$ wurde bei 7 Fundorten ebenfalls nur in Spuren ermittelt, in den übrigen 6 Fällen fehlt derselbe gänz-

1) Eine quantitative Bestimmung der halbgebundenen und gebundenen Kohlensäure erfordert bedauerlicherweise größere Wassermengen, deren Beschaffung uns mit Rücksicht auf die Höhenlage der Fundorte unmöglich war; hiedurch geht leider eine für die biologische Seite unserer Untersuchungen sehr wertvolle Angabe verloren.

$\left.{ }^{2}\right)$ Siehe Anmerkung in der Tabelle. 


\begin{tabular}{|c|c|c|c|c|c|c|}
\hline \multirow{2}{*}{ Wasserbecken } & \multicolumn{4}{|l|}{ hweis } & \multirow{2}{*}{$\begin{array}{c}\text { Ent- } \\
\text { faltung } \\
\text { der } \\
\text { Wasser- } \\
\text { flora } \\
\end{array}$} & \multirow{2}{*}{$\begin{array}{l}\text { Entomostraken- } \\
\text { plankton }\end{array}$} \\
\hline & $\mathrm{NH}_{3}$ & $\mathrm{CO}_{2}$ & K $\mathrm{s}$ & $\mathrm{Na}$ & & \\
\hline $\begin{array}{c}\text { Unterer Se bise e } \\
\text { Yord-Tirol; } 2229 \mathrm{~m} \text { ü. } \\
\text { d. Neere. Seicht. } \\
\text { Temp. } 13,6^{\circ} \mathrm{C} .\end{array}$ & & Spuren & + & + & $\begin{array}{l}\text { Reich an } \\
\text { Algen }\end{array}$ & $\begin{array}{l}\text { Homa, Chydorus, } \\
\text { Macrothrix; } \\
\text { Dinptomus, Cy- } \\
\text { clops. }\end{array}$ \\
\hline $\begin{array}{c}\text { Oberer Sonntags - } \\
\text { karse e } \\
\text { Nord-Steiermark; } 2036 \mathrm{~m} \\
\text { ü. d. Neere. ? m. } \\
\text { Temp. } 3,2^{\circ} \mathrm{C} \text {. }\end{array}$ & & Spuren & + & + & Arm & $\begin{array}{l}\text { Acroperus, Bos- } \\
\text { mina; Cantho- } \\
\text { camplus. }\end{array}$ \\
\hline $\begin{array}{c}\text { Unterer Sonntags - } \\
\text { karsee } \\
\text { Nord-Steiermark; } 1930 \mathrm{~m} \\
\text { i. d. Meere. ? m. } \\
\text { Temp. } 9^{\circ} \mathrm{C} \text {. }\end{array}$ & 1 & Spuren & + & + & $\begin{array}{c}\text { Reich an } \\
\text { Algen }\end{array}$ & $\begin{array}{l}\text { Acroperus, Alona; } \\
\text { Canthocamptus, } \\
\text { Cyclops. }\end{array}$ \\
\hline $\begin{array}{c}\text { Oberer Gigla chs e e } \\
\text { Nord-Steiermark; } 1960 \mathrm{~m} \\
\text { ii. d. Meere. ? m. } \\
\text { Temp. } 10^{\circ} \text { C. } \\
\end{array}$ & 1 & Spuren & + & + & $\begin{array}{c}\text { Reich an } \\
\text { Algen }\end{array}$ & $\begin{array}{l}\text { Acroperus, Alona, } \\
\text { Bosmina, Chydo- } \\
\text { rus;Cyclops(Har- } \\
\text { pacticidengenus). }\end{array}$ \\
\hline $\begin{array}{l}\text { I ut terbergersee } \\
\text { Nord-Tirol; } 2483 \mathrm{~m} \text { ü. } \\
\text { d. Meere. ? m. } \\
\text { Temp. } 13,6^{\circ} \mathrm{C} \text {. }\end{array}$ & ; & Spuren & + & + & Arm & $\begin{array}{c}\text { Alona, Chydorus; } \\
\text { Cyclops. }\end{array}$ \\
\hline $\begin{array}{c}\text { Unterer Plenderle- } \\
\text { see } \\
\text { Nord-Tirol: } 2250 \mathrm{~m} \text { ü. } \\
\text { d. Meere. ? m. } \\
\text { Temp. } 7,3^{0} \mathrm{C} .\end{array}$ & & Spuren & + & + & Arm & Chydorus;Cyclops. \\
\hline $\begin{array}{c}\text { K a mpspitzse e } \\
\text { Nord.Steiermark; } 2200 \mathrm{~m} \\
\text { ii. d. Meere. ? seicht. }\end{array}$ & 7 & Spuren & +1 & + & Arm & $\begin{array}{l}\text { Alonella; Cantho- } \\
\text { camptus, Cyclops. }\end{array}$ \\
\hline
\end{tabular}

\begin{tabular}{|c|c|c|c|c|c|c|}
\hline 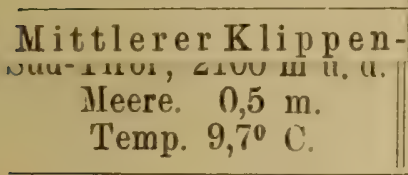 & 1 & Spuren & + & $t$ & Fehlt! & Chydorus. \\
\hline $\begin{array}{c}\text { B o ës e e } \\
\text { Süd-Tirol; } 2282 \mathrm{~m} \text { ü. d. } \\
\text { ILeere. Tief. } \\
\text { Temp. } 10,5^{\circ} \mathrm{C} .\end{array}$ & Spuren & Spuren & + & + & $\begin{array}{l}\text { Reich an } \\
\text { höheren } \\
\text { Wasser- } \\
\text { pflanzen } \\
\text { u. Algen } \\
\end{array}$ & $\begin{array}{l}\text { Chydorus, Daph- } \\
\text { nia; Cyclops, } \\
\text { Diaptomus. }\end{array}$ \\
\hline $\begin{array}{c}\text { Grïinsee } \\
\text { Salzburg; } 1699 \mathrm{~m} \text { ü. d. } \\
\text { Ileere. } 32,2 \mathrm{~m} . \\
\text { Temp. } 8{ }^{\circ} \mathrm{C} .\end{array}$ & & Spuren & + & & $\begin{array}{c}\text { Reich an } \\
\text { Algen }\end{array}$ & $\begin{array}{l}\text { Acroperus, Alona, } \\
\text { Chydorus; Cyclops. }\end{array}$ \\
\hline $\begin{array}{l}\text { K r u m m se e } \\
\text { Tirol; } 557 \mathrm{~m} \text { ii. d. } \\
\text { IIeere. } 2,5 \mathrm{~m} . \\
\text { Temp. } 17,25^{\circ} \mathrm{C} .\end{array}$ & Spuren & Spuren & + & & $\begin{array}{l}\text { Sehr reich } \\
\text { an höhereu } \\
\text { W'asser- } \\
\text { pflanzen } \\
\text { u. Algen }\end{array}$ & $\begin{array}{l}\text { Acroperus, Alona, } \\
\text { Bosmina, Cerio- } \\
\text { daphnia, Diapha- } \\
\text { nosoma, Grapto- } \\
\text { leberis, Peracan- } \\
\text { tha, Sida; Cyclops, } \\
\text { Diaptomus. }\end{array}$ \\
\hline
\end{tabular}

1) Ob freies oder 



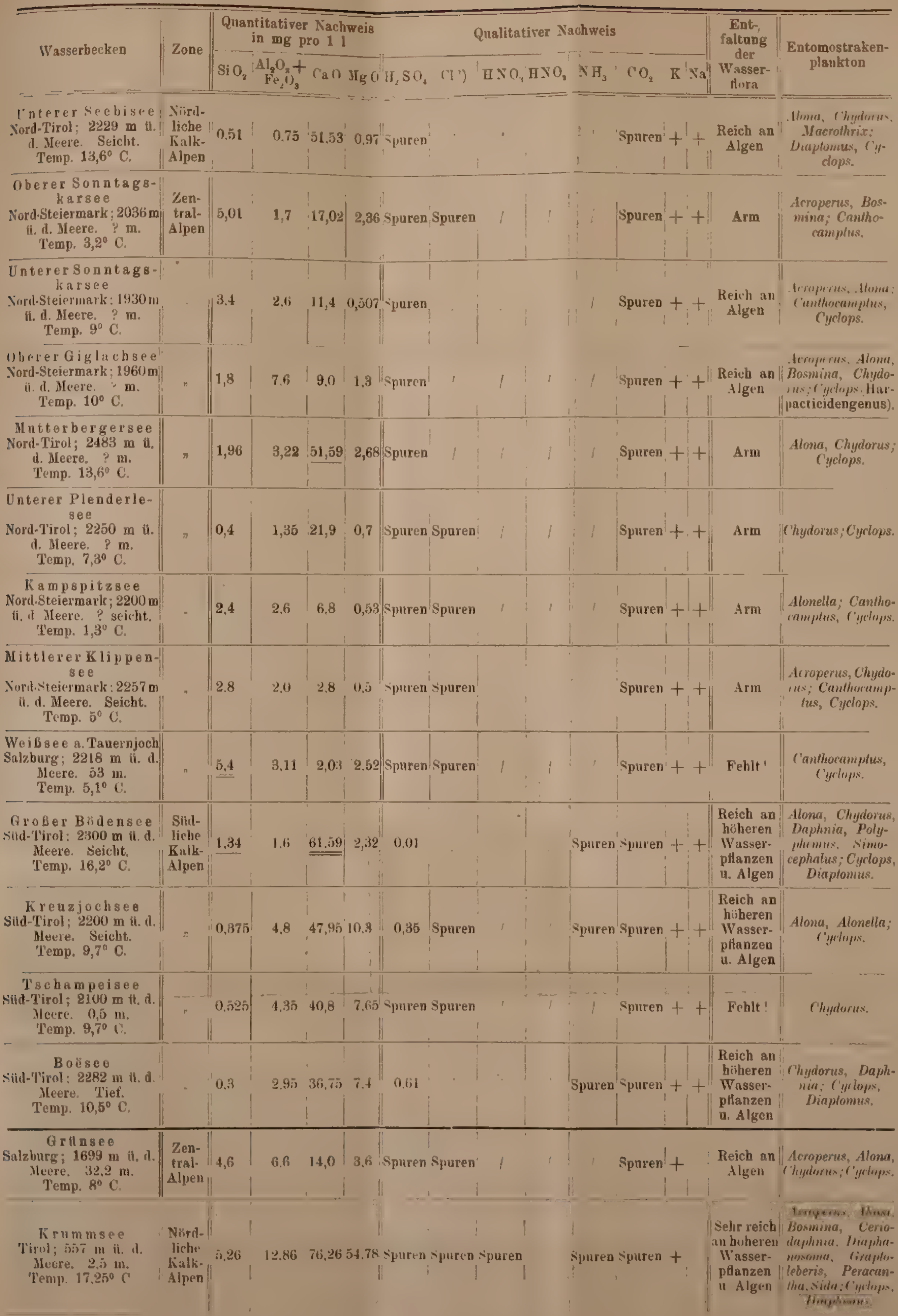

') Ob freies oder an Alkalien gebundenes $\mathrm{Cl}$ ist in den Anulyscn nicht betont. 

lich. Der Befund erscheint ohne wesentliche Bedeutung, wie auch endlich

5. die ständige Anwesenheit von Kalium (K) und Natrium (Na) nichts Auffallendes darstellt.

Welche Ergebnisse liefert die quantit a $\mathrm{t}$ ive Analyse?

1. Der Gehalt an Kieselsäure $\left(\mathrm{Si}_{2}\right)$, welcher sich zwischen den Extremen von 0,3 und $5,4 \mathrm{mg}$ pro Liter bewegt, bleibt im allgemeinen bei den in der Zone der Nördlichen und Südlichen Kalkalpen liegenden Hochgebirgsseen gegenüber jenen der Zentralalpen (Urgebirge) zurück, erreicht im Weißsee (Granitgneis) das Naximum und sinkt im Boësee (Dolomit) auf das Minimum herab. Bei weiteren sieben in der Zentralalpenzone gelegenen Seen übersteigt er noch immer jenen der fünf Wasserbecken der Nördlichen und Südlichen Kalkalpenzone. Dieses Verhalten entspricht somit der Voraussetzung, daß der geologische Bau des Fundortes einen Schluß auf das Vorherrschen oder Zurücktreten des Kieselsäuregehaltes zuläßt. Der mitten im Dolomitgebiet Südtirols gelegene Große Bödensee weist jedoch mit $1,34 \mathrm{mg} \mathrm{Si} \mathrm{O}_{2}$ einen wesentlich höheren Gehalt dieses Stoffes auf als der in Zentralgneismassen eingebettete Untere Plenderlesee der Ötztaler Alpen mit nur $0,4 \mathrm{mg}$ Si $\mathrm{O}_{2}$ pro Liter; außerdem zeigt die Tabelle, daß noch zwei Fundorte der Zentralalpenzone - nämlich der Obere Giglachsee und der Müutterbergersee - einen sehr geringen Kieselsäuregehalt (unter $2 \mathrm{mg}$ ) besitzen, somit nach diesem Gehalt sich kaum als Urgesteinsbecken dokumentieren. Dieser Befund beweist uns, daß es in manchen Fällen verfehlt ist, aus der geologischen Beschaffenheit des Seegebietes direkten Rückschluß auf das Dominieren des Kieselsäuregehaltes zu ziehen. Die Ursachen dieser von vorneherein nicht abzusehenden Erscheinung dürften wahrscheinlich in spezifisch mineralogischen Verhältnissen des betreffenden Fundortes liegen und vermutlich in der Größe des Löslichkeitsgrades der von den Gewässern des Zuflusses durchströmten Gesteinsarten zu suchen sein.

2. Aluminium- und Eisenoxyd $\left(\mathrm{Al}_{2} \mathrm{O}_{3}+\mathrm{Fe}_{2} \mathrm{O}_{3}\right)$ tritt in den 13 untersuchten Hochgebirgsseen ohne nachweisbare Gesetzmäßigkeit in sehr variablen Mengen auf.

3. Dasselbe gilt vom Magnesiagehalt (Mg O). 
4. I) größte Kalkreichtum (Ca O) entfällt auf den Großen Bödensee im Dolomitgebiet Südtirols mit $61,59 \mathrm{mg}$ pro Liter; ihm schließen sich die übrigen vier den Kalkalpenzonen zugehörigen Wasserbecken mit einem Gehalt von $36,75-51,53 \mathrm{mg}$ Ca $O$ pro Liter an. Acht Seen der Zentralalpenzone bleiben - diesbezüglich verglichen - weit hinter diesen Gewichtsmengen an Kalk zurück. Das Ergebnis der Analysen entspricht daher vollkommen den Vorstellungen von der Beziehung des Kalkgehaltes der Seewässer zum geologischen Charakter der Fundorte. Dazu steht nun das Verhalten des im Gneisgestein liegenden Mutterbergersees der Stubaier Alpen im schroffen Widerspruch; der See übertrifft mit seinem erheblichen Kalkgehalt von $51,59 \mathrm{mg}$ nicht weniger als vier von fünf untersuchten Wasserbecken aus den Kalkalpenzonen.

Als wichtigstes Ergebnis unseres Versuches, die chemische Beschaffenheit des Wassers einer Anzahl von. Hochgebirgsseen festzustellen, darf demnach hervorgehoben. werden, dab Reichtum oder Armut an Kieselsäure- und Kalkgehalt zwar in vielen Fällen aus dem geologisehen Aufbau des Gebirgeszu ersehließen ist, ein Dominieren oder Zurücktreten dieser Stoffejedoch keineswegs an jederbeliebigen Fundstellenachdiesem Gesichtspunktbeurteiltwerdenkann; erst die Analysegibt darüber endgültigen und einwandfreien Auf$\operatorname{sehlu} B$.

Den 13 aus der Region des Hochgebirges stammenden Seewasseranalysen haben wir des Vergleiches wegen noch zwei angeschlossen, welche von Becken gewonnen wurden, die sich in verschiedenen Höhenlagen der Waldregion befinden. Von diesen beiden fügt sich die Analyse des Grünseewassers vollkommen in die Reihe der vorhergenannten Hochgebirgsgewässer. Der Grünsee, in einer Höhe von $1699 \mathrm{~m}$ ü. d. M. nahe an der Waldgrenze gelegen, zählt zu den chemisch ,reinen “1), eines stärkeren Pflanzenwuchses entbehrenden und nicht warm temperierten Wasserbecken der Zentralalpen (Gneis); der quantitative Gehalt an Kieselsäure (4,6 mg pro Liter) entspricht verhältnismäßig hohen, jener an Calciumoxyd (14 mg) verhältnismäßig niederen Werten, wie sich aus der

\section{1) Im Sinne von Mangel an Stickstoffverbindungen.}


tabellarischen Übersicht verglcichsweise ergibt. Von sämtlichen untersuchten Becken ganz verschieden verhält sich dagegen das Ergebnis der Analyse aus dem Krummsee, welcher im waldreichen Gebiete des niedrigen Mittelgebirges (כ̌ $7 \mathrm{~m}$ ü. d. M.) der Kalkalpen gelegen ist. Der quantitative Nachweis der einzelnen Stoffe (Verbindungen) zeigt durchwegs auffallend hohe Zahlen, ganz besonders für die Aluminium-EisenOxyde (12,86 mg pro Liter) und für das Magnesiumoxyd (54,78 mg); mit dem Gehalt von 76,26 mg Calciumoxyd übertrufft dieses Gewässer ebenfalls alle anderen. Dem letzten steht - der geologisch-petrographischen Beschaffenheit des Fundortes entsprechend --ein bedeutendes Zurücktreten der Kieselsäure $(5,26 \mathrm{mg})$ gegenüber, jedoch ist dic Mienge wiederum außergewöhnlich hoch im Vergleiche zum Kieselsäuregehalt der fünf in der Hochgebirgsregion gelegenen Seen der Kalkalpenzonen (Seebisee, Bödensee, Kreuzjochsee, Tschampeisee, Boësee). Im übrigen gehört der Krunmsee zu den stärker verunreinigten (Cl-, $\mathrm{H} \mathrm{N} \mathrm{O}_{3^{-}}$- und $\mathrm{N} \mathrm{H}_{3}$-hältigen), an Pflanzenwuchs sehr reichen und ausgesprochen warm temperierten Wasserbecken. Wenn die hervorgehobene Sonderstellung bezüglich der chemischen Eigenschaften des Krummseewassers mit der niederen Höhenlage und dem Pflanzenreichtum der Fundstelle in Zusammenhang gebracht werden kann, so würde sie diesen See sehr gut als Typus eines Wasserbeckens der Waldregion charakterisieren. Es soll jedoch genügen, ohne Erklärungsversuch auf den tatsächlich bestehenden Unterschied im Chemismus der von uns bisher untersuchten Ostalpenseen hingewiesen zu haben. -

Die Prüfung auf das chemische Verhalten der Hochgebirgsseewässer hat ferner ein charakteristisches Merkmal aufgedeckt, welchem wahrscheinlich eine größere biologische Bedentung zukommt, als bisher erkannt wurde ${ }^{1}$ ). Es zeigt sich eine deutliche Scheidung in ammoniakfreie und ammoniakhaltige $\left(\mathrm{N} \mathrm{H}_{3}\right)$ Wasserbecken. Die Anwesenheit dieser Stickstoffverbindung steht in auffälliger Beziehung zur reicheren Entfaltung der Wasservegetation bezw. zum Vorkommen höherer Wasserpflanzen. Die Herkunft des Ammoniaks ist noch nicht völlig geklärt; es kann hiefür die direkte Düngung (durch weidendes Almvieh) oder die Zersetzung abgestorbener Organismen verantwortlich gemacht werden. Da aber im Hochgebirge die vegetationsarmen Seen weit häufiger vertreten sind als die vegetationsreichen und diese Erscheinung nach

1) Für die Seen Schwedens hat Naumans, E. [op. cit.] die Einflüsse der Stickstoffverbindungen (Düngung) untersucht und auf dieser Basis „oligotrophe" und ,eutrophe" Seetypen unterschieden. 
unseren Beobachtungen wiederum ganz unabhängig von der Düngungsmöglichkeit durch Weidevieh besteht, so wird als primäre Ursache des Ammoniakgehaltes die reiche Wasserflora angesprochen werden können. In diesen pflanzenreichen, ammoniakhaltigen Hochgebirgsseen ist stets eine arten- und individuenreiche Cladoceren- und Copepodenfauna $\mathrm{zu}$ erwarten, während die pflanzenarmen Wasserbecken sich als bedeutend faunenärmer erweisen; eine diesbezügliche Durchsicht der bisher untersuchten Hochgebirgsseen läßt diesen Zusammenhang recht deutlich erkennen. Es besteht kein Zweifel, daß ein reicheres Vorkommen grüner Wasserpflanzen im Hochgebirgssee als ausgiebige Quelle der Sauerstoffversorgung in Betracht kommt und im Chemismus derartiger Seewässer wahrscheinlich eine viel größere Entscheidung spielt, als sie die Unterschiede im Kalk- oder Kieselsäuregehalt auf das Vorkommen der Copepoden- und Cladocerenarten auszuüben vermögen. In diesem Sinne weisen die faunistischen Ergebnisse darauf hin, daß z. B. das Auftreten der Genera Heterocope und Diaptomus im Hochgebirge mit der Reihe der Faktoren Ammoniakgehalt-Wasserflora-Sauerstoffbedürfnis in einem direkten Abhängigkeitsverhältnisse stehen dürfte. Weiteren Untersuchungen fällt die Aufgabe zu, dem hier kurz angedeuteten Zusammenhang besondere Aufmerksamkeit zu schenken.

\section{A b s chnitt.}

\section{Geographisch-geologische Charakteristik, Begrenzung und Einteilung der Ostalpen. Seenverzeichnis.}

Da im nächsten Abschnitte dieser „Studien“ der Versuch gemacht werden soll, eine zusammenfassende Darstellung der bisher auf ihre Cladoceren- und Copepodenfauna untersuchten Ostalpenseen zu geben, welche außerhalb der Schweizer Grenze fallen, wird es nicht überfiüssig sein, zunächst eine allgemeine Charakteristik des Gebietes vorauszuschicken und auch einige auf die hydrobiologische Beurteilung der Wasserbecken bezugnehmende Gesichtspunkte zu besprechen.

Für die zwei Teile, in welche der gesamte, vom Ligurischen Meer bis zur Donau bei Wien reichende Zug der Alpen zerfällt, ist die Be- 
zeichnung „Westalpen“ und „Ostalpen“ allgemcin gebrälichlich ${ }^{1}$ ). Es handelt sich in diesem Falle keineswegs um eine künstliche Gruppierung, vielmehr ist sie durch die Entstehungsgeschichte geologisch begründet; das gänzliche Fehlen einer südlichen Kalkalpenzone im westlichen Bogen (Westalpen) scheidet den östlichen Bogen (Ostalpen) auffällig ab. Den genaueren Verlauf der Grenze bezeichnet die Linie: Bodensee-RheinHinterrhein-Splügenpaß_Comersee-Luganersee-Lago maggiore.

Das Gebiet der Ostalpen umfaßt drei deutliche Zonen, welche gemäß der Verschiedenheit in den Hauptmassen des Gesteines, aus dem sie bestehen, unter dem Namen Nördliche Kalkalpen, Zentralalpen und Südliche Kalkalpen bekannt sind. Rechnet man den zwischen den zwei erstgenannten Zonen eingeschobenen Streifen der Schieferalpen (= Übergangsgebirge) zu den Zentralalpen und das zwischen den Südlichen Kalkalpen und den Zentralalpen eingeschaltete "Klagenfurter Becken“, ein Kontaktgebiet, zu den Südlichen Kalkalpen, so besteht für die Zentralalpen folgende Nord- und Südgrenze ${ }^{2}$ ):

1. Grenze gegen die Nördlichen Kalkalpen: Bodensee-RheinPrättigau (Landquart)-Klosters-Schlappeiner Joch-Gargellental-Montavon bis Schruns-Silbertal-Dalaas-Klostertal-Arlberg-Stanzertal-Inntal bis Wörgl-Söll-EllmauSt. Johann in Tirol-Hochfilzen-Leogang-Saalfelden-Filzensattel-Bischofshofen-Fritztal-Mandlingpaß_Ennstal bis Admont-Längseinsenkungen parallel zum Palten- und Liesingbach über Eisenerz und Prebichl bis zum Mürztal-Preiner Gschaidsattel-Payerbach-Gloggnitz-Neunkirchen-Schwarzau-Baden-Wien (Donau).

2. Grenze gegen die Südlichen Kalkalpen: Lago maggiore (Luino)Lugano-Comersee bis ans Nordende-Veltlintal-ApricapaßEdolo-Val Camonica bis Breno-Croce Dominipaß-Nordende des Idrosees-Roncone-Val Rendena-S. Maria di CampiglioSulzberg (Val di Sole)-Nocetal-Val di Pescara-Castrirsattel-Ultental_Lana (Meran) - Naifpaß - Sarnthein - Villanderalpe-Eisacktal bis Franzensfeste-Pustertal-Toblacherfeld-Drautal bis Villach-Ossiachersee-Glantal bis St. VeitGuttaring im Gurktal-Brückl-Haimburg-St. Andrä im

1) Den folgenden Zeilen diente hauptsächlich die vortreffliche Abhandlung von A. BöHм [1887] „Einteilung der Ostalpen" als Grundlage.

$\left.{ }^{2}\right)$ Siehe die beiliegende Karte der Ostalpen. 
Lavanttal-Lavamünd-Unterdrauburg-Mißlingtal bis St.

Leonhard-Weitenstein-Windisch Feistritz-Marburg (Drau).

In den drei genannten Hauptzonen der Ostalpen hat eine Gruppierung nach folgenden Gebirgszügen Geltung:

I. Nördliche Kalkalpen:

1. Allgäuer Alpen (Rhätikon, Bregenzer Wald, Lechtaler Alper).

2. Nordtiroler Kalkalpen (Altbayerische Alpen und Nordtiroler Kalkalpen im engeren Sinne).

3. Salzburger Kalkalpen (Waidringer und Berchtesgadner Alpen; Salzkammergut).

4. Österreichische Kalkalp€n.

II. Zentralalpen:

1. Rhätische Alpen.

2. Salzburger Schieferalpen (Tuxer und Kitzbühler Alpen).

3. Hohe Tauern (Zillertaler Alpen und Hohe Tauern im engeren Sinne).

4. Niedere Tauern (Radstätter, Schladminger, Rottenmanner Tauern).

5. Eisenerzer Alpen.

6. Norische Alpen (Gurktaler, Lavanttaler Alpen, Bacher-, Posruckgebirge).

7. Cetische Alpen (Gleinalpen, Grazerbucht-, Fischbacher Alpen).

III. Südliche Kalkalpen:

1. Lombardische Alpen (Luganer und Bergamasker Alpen).

2. Etschbuchtgebirge (Brescianer-, Brenta-, Sarca-, Nonsberggruppe; Vizentinische Alpen).

3. Südtiroler Hochland (= Dolomiten).

4. Venetianer Alpen.

5. Julische Alpen (Raibler und Steiner Alpen).

6. Karnische Alpen (Gailtaler Alpen, Hauptkette, Karawanken).

7. Klagenfurter Becken.

Wie eingangs erwähnt wurde, sind jene Seen, welche nach den vorhergehenden Angaben zwar im Ostalpengebiete, jedoch noch innerhalb der Schweizer Landesgrenze liegen, in diesen „Studien" nicht berücksichtigt; Stingelin [1908] und Thiebaud [1915] haben über die Cladoceren- und Copepodenfauna dieser Wasserbecken ohnedies zusammenfassend berichtet; dagegen schien es uns nicht wertlos, die bestehende Lücke einer übersichtlichen Zusammenstellung für den wesentlich größeren Bereich des übrigen Ostalpengebietes auszufüllen. 
85 der im folgenden Abschnitte aufgezählten Seen fallen in die Zone der Nördlichen Kalkalpen. Hiezu ist zu bemerken, daß der Bodensee des Vergleiches wegen miteinbezogen erscheint; ferner befinden sich noch eine Anzahl der im bayerischen Alpenvorlande gelegenen Seen darunter, welche, strenge genommen, außerhalb der von den Geographen gezogenen Nordgrenze der Kalkalpen liegen, für unsere faunistische Darstellung aber von Wichtigkeit sind (z. B. Starnbergersee, Simssee, Ammersee, Chiemsee). Zur Zentralalpenzone gehören 58 der untersuchten Seen. Auch hier wurde der Neusiedlersee, obwohl nicht mehr ins Alpenbereich fallend, der Vollständigkeit und des Vergleiches wegen berücksichtigt. Auf die Zone der Südlichen Kalkalpen endlich entfallen 54 Seen; zu ihnen zählt der von Iмноғ untersuchte „Tihoja“see in Kärnten, dessen genauere geographische Lage von uns nicht ermittelt werden konnte. Utber die Verteilung der aufgezählten Seen (Fundorte) in den Zonen der Ostalpen gibt die beiliegende Karte Aufschluß.

Da die zonare Einteilung der Ostalpen lediglich nach den Hauptmassen des dominierenden Gesteines getroffen ist, so kann sie nur in großen und groben Zügen die geologisch-petrographischen Unterschiede der drei als Nördliche und Südliche Kalkalpen und Zentralalpen bezeichneten Gebiete angeben; dem Überwiegen der "Kalkgesteine" in den zwei ersten Zonen steht das Vorherrschen der sogenannten „Ur-

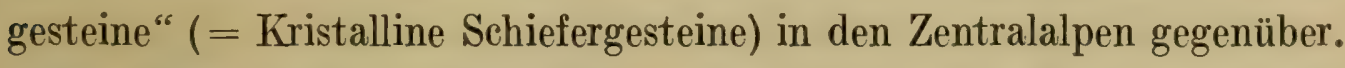
Вӧнм [1887, p. 252] sagt hierüber: „Diese Gebirgszonen sind jedoch nicht ganz mit den betreffenden geognostischen Zonen identisch, sondern es finden mitunter Gesteine derselben auch jenseits der angegebenen Grenzen eine lokale Verbreitung." Dasselbe betont Heritsch [1921, p. 12]: ...,,weite Gebiete der Zentralalpen werden von kalkigen Serien aufgebaut und andererseits findet man in den Kalkalpen viele kalkfreie Gesteine als wesentliche Bauelemente. Doch ist immerhin der Unterschied so weitgehend, daß Kalk- und Zentralalpen einander gegenübergestellt bleiben müssen und daß man mit Recht von einer Grenze zwischen beiden reden muß."

Wenn nun unsere in den nächstfolgenden Abschnitten enthaltene Zusammenstellung die Lage der Ostalpenseen auch in bezug auf diese drei genannten geologisch-petrographischen Hauptzonen berücksichtigt, so will damit unter einem versucht sein, eine bisher nicht beachtete Ursache, welcher für die horizontale Verbreitung der Cladoceren und Copepoden im Ostalpenbereiche eine Bedeutung zukommen könnte, aufzuzeigen. Den vermutlichen Zusammenhang hat bereits Stever [1910, 
Planktonkunde p. 28] in dem Satze ausgesprochen: „Wenn nun tatsächlich das Plankton qualitativ oder quantitativ von der chemischen Zusammensetzung des Wassers abhängig ist, dann wird es vielleicht möglich sein, das Plankton irgendeines Sees mit dem geologischen Charakter der Gegend, in der der See liegt, in nähere Beziehung zu bringen." Es ist überflüssig, hervorzuheben, daß eine derartige Beziehung nicht nur für das Plankton im strengen Sinne, sondern auch ebensogut für Litoral- oder Grundbewohner erwartet werden könnte.

Nach dem früher zitierten Hinweis wird es im Einzelfall, d. h. in Anwendung auf eine bestimmte Fundstelle (See), fast stets notwendig sein, die geologische Beschaffenheit der Umgebung des betreffenden Wasserbeckens (Zufluß- und Niederschlagsgebiet) genauer festzustellen, als dies durch die Bezeichnung der "Zone“ allein geschieht; dieser Forderung suchten wir in der Angabe geologisch-hydrographischer Daten einigermaßen gerecht zu werden, doch dürfen sie nur bei einer beschränkten Anzahl von Seen (21) zu unseren eigenen Beobachtungen gezählt werden.

Es sei ferner darauf hingewiesen, daß die in diese "Studien" aufgenommenen Ostalpenseen dem System folgender Hauptwasseradern angehören:

I. dem Rhein (Nordsee):

Bodensee;

Lünersee, Tilisunasee, Todtalpsee (III);

Gafiensee, Garschinasee, Partnunersee (Landquart);

II. der Donau (Schwarzes Meer):

Alpsee bei Immenstadt, Sonthofersee (Iller);

Alpsee bei. Füßen, Bannwaldsee, Hopfensee, Piansee, Schwansee, Unterer Seebisee, Weißensee bei Füßen (Lech: 7);

Achensee, Ammersee, Badersee Eibsee, Niittersee am Fernpaß, Rissersee, Staffelsee, Starnbergersee, Walchensee, Weißensee am Fernpaß, Wildsee bei Seefeld (Isar: 11);

Berglsteinersee, Chiemsee, Faistenauer Hintersee, Hinterer und Vorderer Finstertalersee, Grünsee, Hechtsee, Hintersee bei Ramsau, Hintersteinersee, Klammjochsee, Königssee, Kreuthsee, Krummsee, Lansersee, Lansermoorsee, Lauterersee, Lichtsee, Mariasteinersee, Mutterbergersee, Nassereithersee, Niedertrumersee, Obersee beim Königssee, Piburgersee, Pillersee, Oberer und Unterer Plenderlesee, Unterer Pochartsee, Reintalersee, Reithersee, Schlickersee, Schliersee, Schwarzsee bei Kitzbühel, Schwarzensee im Zillertal, Simssee, Sistransersee, 
Spitzingsee, Tegernsee, Thumsee, Tiersee, Unterer Torjochsee, Wallersee, Weißensee am Tauernjoch, Wildsee am Wildsecloder, Zellersee, Zireinersee (Inn: 45);

Altausseersee, Attersee, Augstsee, Augstwiesensee, Fuschelsee, Vorderer Gosausee, Grundlsee, Hallstättersee, Igelsee beim Attersee, Kammersee bei Aussee, Küöllensee, Krottensee, Hinterer und Vorderer Lahngangsee, Vorderer Langbathsee, Mittersee am Schafberg, Mondsee, Münichsee, Nussensee, Vorderer Oberbergersee, Ödensee, Offensee, Röthelsee, Schwarzsee am Schafberg, Sch warzensee beim Ödensee, Sommersbergersee, Toplitzsee, Traunsee, Wildensee, Wolfgangsee (Traun: 30);

Filzsee, Gaishornsee, Oberer Giglachsee, Kampspitzsee, Oberer Klaftersee, Mittlerer Klippensee, Leopoldsteinersee, Oberer und Unterer Sonntagskarsee (Enns: 9);

Lunzer Mlittersee, Lunzer Obersee, Lunzer Untersee (Ybbs);

Erlaufsee (Erlaf);

Neusiedlersee (Raab);

Anderlesee, Kleiner See südlich vom Anderlesee, Kleiner See westlich vom Anderlesee, Auerlingsee, Berghaustümpelsee. Großer und Kleiner Bödensee, Brennsee, Faakersee, Fresenhalssee, Gösselsdorfersee, Jeserzsee, Keutschachersee, Klopeinersee, Königsangersee, Lambrechtersee, Oberer und Unterer Lanischsee, Leonhardsee, Großer und Kleiner Magdalenensee, Millstättersee, Unterer und Oberer Mühldorfersee, Ossiachersee, Presseckersee, Raiblersee, Saualmsee, Saureggersee, Schwarzsee an der Turracherhöhe, Seebachsee, Speiksee, Tihojasee, Großer Turrachersee, Vassachersee, Weißensee bei Greifenburg, Oberer und Unterer Weißenfelsersee, Worstnigsee, Wörthersee, Zirmsee (Drau: 41);

Veldessee, Wocheinersee (Save).

III. dem Po (Adria):

Foscagnosee, Fraëlesee, Gardasee, Idrosee, Iseosee, Ledrosee, Santa Massenzasee, Toblinosee (8);

IV. der Etsch (Adria):

Ceisee, Dürrensee, Haidersee, Kalterersee, Kreuzjochsee, Lagoraisee, Lasessee Loppiosee, Großer und Kleiner Miontigglersee, Pfitscherjochsee, Piazzesee, Pragsersee, Santosee, Seraiasee, Stellunesee, Terlagosee, Toblachersee, Tovelsee, Tschampeisee $(20)$; 
V. der Brenta (Adria):

Caldonazzosee, Lavaronesee, Levicosee;

VI. dem Piave (Adria):

Alleghesee, Boësee, Misurinasee, Revinesee, S.-Crocesee;

VII. dem Tagliamento (Adria):

Cavazzosee, S.-Danielesee.

Im folgenden Abschnitte fanden nur solche Wasserbecken Aufnahme, die ohne Zweifel als „Seen“ gelten müssen; von der rein äußerlichen Eigenschaft der Größe wird das Wesen des Begriffes natürlich nicht abhängig sein; wir verstehen darunter jedes natürlich gebildete, stehende Binnengewässer von perennierender Dauer und schließen künstliche, temporäre, der Austrocknung unterworfene Wasseransammlungen (Teiche, Sümpfe, Tümpel, Lachen) aus unserer Zusammenstellung aus. Für die Seen der Alpen steht bekanntlich das entwicklungsgeschichtliche Moment ganz besonders im Vordergrund; denn ihre Existenz ist mit dem Glazialphänomen auf das innigste verknüpft und bei einer überwiegenden Zahl als unmittelbare Folgeerscheinung der Eiszeitwirkungen zu betrachten. Nach dem Gesichtspunkte der Entwicklungsgeschichte werden demnach zwei Haupttypen unterschieden:

1. Aufschüttungsbecken oder Stauseen. Sie entstanden durch Bergsturz (z. B. Alleghesee, ? Grünsee im Stubachtale, Obersee beim Königssee, Pochartsee bei Gastein, ? Santa Crocesee bei Belluno) oder durch Muhrbruch und Schuttkegelbildung von Wildbächen (z. B. Haidersee bei Mals, Gaishornsee, Reintalersee, Wildsee bei Seefeld) oder durch Terrassenschotter (z. B. Achensee, Alpsee bei Immenstadt, Plansee, Zellersee) oder durch Moränendämme einstiger Gletscher (zahlreiche Beispiele).

2. Eintiefungsbecken oder Echte Felsbecken. Sie entstanden durch die Erosionstätigkeit der Gletscher (z. B. Finstertalerseen, Giglachseen, Pfitscherjochseen, Sonntagskarseen, Weißsee im Stubachtale usw.) oder dureh Einbruch bezw. Einsturz bei Auflösung des Gesteines (z. B. Eibsee, Seen am Fernpaß, Spitzingsee).

Böнм [1886!], welcher die Gesamtsumme der Seen des Alpenzuges schätzungsweise mit gut 5000 veranschlagte, zählte auf Grund des ihm zur Verfügung stehenden Kartenmateriales im Ostalpenbereich 2460 Seen. Es besteht kein Zweifel, daß diese Angabe weit hinter der tatsächlichen Zahl zurückbleibt; man bedenke, welche Lücken erst in letzter Zeit 
durch die kartographischen Darstellungen rieler Hochgebirgsregionen ausgefüllt wurden und um wieviel die von Böнм genannte Zahl durch die unbenannten, in entlegeneren und schwieriger zugänglichen Bergregionen befindlichen Seen zu erhöhen wäre! Daraus mag nun hervorgehen, daß die 197 in diesen "Studien" aufgezählten Wasserbecken einen bescheidenen Bruchteil repräsentieren, da $B$ aber auch andererseits der hydrobiologischen Erforschung der Ostalpenseen noch ein grobes Stück Arbeit bevorsteht, ehe sie abschliefende Urteile fällen darf, die dann des rorwiegend spekulativen Beigeschmackes entbehren werden. Die vorliegenden „Studien" können daher nur den Anspruch auf eine erste Grundlage erheben, welche verschiedene Wege weisen soll, die zur Erweiterung und Vertiefung unserer Kenntnisse über die Hydrobiologie der Ostalpenseen beitragen.

\section{Seenverzeichnis.}

Die Numerierung stimmt mit den auf der beiliegenden Karte eingetragenen Ziffern (= Fundorte) überein.]

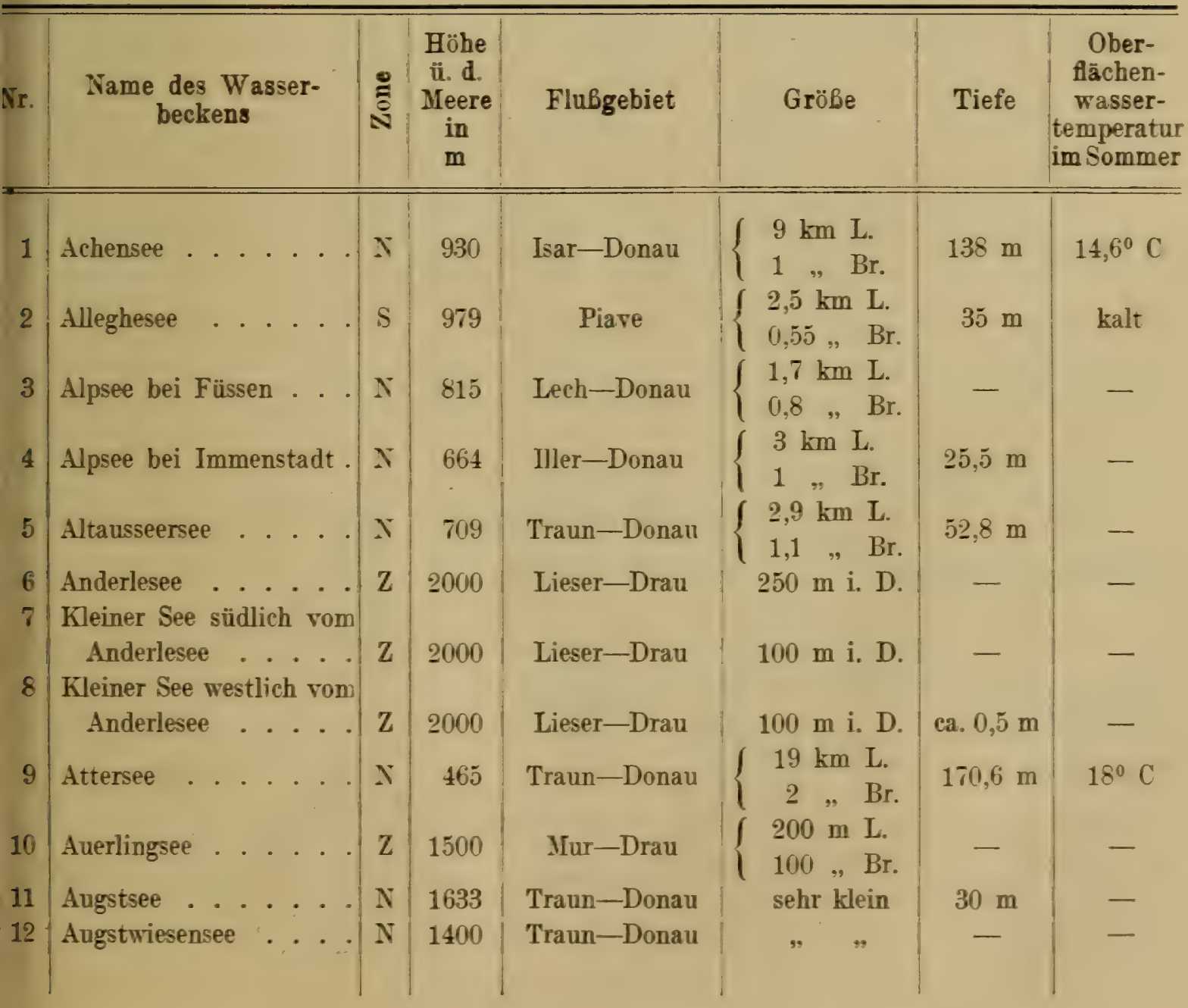




\begin{tabular}{|c|c|c|c|c|c|c|c|}
\hline Nr. & $\begin{array}{c}\text { Name des Wasser- } \\
\text { beckens }\end{array}$ & : & $\begin{array}{c}\text { Höhe } \\
\text { ü. d. } \\
\text { Meere } \\
\text { in } \\
\mathrm{m}\end{array}$ & FluBgebiet & Größe & Tiefe & \begin{tabular}{|} 
Ober- \\
fläches \\
wasses \\
tempera! \\
im Somn
\end{tabular} \\
\hline 13 & $\ldots \ldots$ & $\mathrm{N}$ & 830 & Isar-Donau & $200 \mathrm{~m}$ i. $\mathrm{D}$. & $15 \mathrm{~m}$ & $10,6^{0}$ \\
\hline 14 & Bannwaldsee ... & $\mathrm{N}$ & 792 & Lech-Donau & $\left\{\begin{array}{l}2 \mathrm{~km} \\
1, \mathrm{Lr}\end{array}\right.$ & $11 \mathrm{~m}$ & 一 \\
\hline 15 & Berghaustümpelsee. . & $\mathrm{Z}$ & 1700 & Lieser-Drau & sehr klein & seicht & - \\
\hline 16 & Berglsteinersee . . & $\mathrm{N}$ & 740 & Inn-Donau & $\left\{\begin{array}{l}300 \mathrm{~m} \mathrm{~L} . \\
200, \mathrm{Br} .\end{array}\right.$ & $1 \mathrm{~m}$ & - \\
\hline 17 & Bodensee ..... & $\mathrm{N}$ & 398 & Rhein & $\begin{cases}69 \mathrm{~km} & \mathrm{~L} . \\
13 & \mathrm{Br}\end{cases}$ & $308 \mathrm{~m}$ & $17,8^{\circ}$ \\
\hline 18 & Großer Bödensee . . & S & 2300 & Drau & klein & seicht & $16,2^{\circ} \mathrm{C}$ \\
\hline 19 & Kleiner Bödensee . . & $\mathrm{S}$ & 2300 & Drau & " & seicht & $18,7^{\circ} \mathrm{C}$ \\
\hline 20 & Boësee . . . . . & S & 2282 & Piave & ca. $100 \mathrm{~m} \mathrm{i.} \mathrm{D.}$ & tief & $10,5^{\circ} \mathrm{C}$ \\
\hline 21 & Brennsee ..... & $\mathrm{Z}$ & 742 & Lieser-Drau & $1,5 \mathrm{~km} \mathrm{~L}$. & 一 & $24^{\circ} \mathrm{C}$ \\
\hline 22 & Caldonazzosee . . . & $\mathrm{S}$ & 449 & Brenta & $\left\{\begin{array}{ccc}4,2 & \mathrm{~km} & \mathrm{~L} . \\
1,7 & , & \mathrm{Br} .\end{array} \mid\right.$ & $49 \mathrm{~m}$ & $24,5^{\circ} \mathrm{O}$ \\
\hline 23 & Cavazzosee . . . & S & 195 & Tagliamento & $\left\{\begin{array}{lll}5 & \mathrm{~km} & \mathrm{~L} . \\
1 & \# & \mathrm{Br}\end{array}\right.$ & $41 \mathrm{~m}$ & - \\
\hline 24 & Ceisee . . . . & $\mathrm{S}$ & 927 & Ceibach-Etsch & $\left\{\begin{array}{l}400 \mathrm{~m} \mathrm{~L} . \\
150, \mathrm{Br} .\end{array}\right.$ & $6,5 \mathrm{~m}$ & - \\
\hline 25 & Chiemsee ..... & $\mathrm{N}$ & 519 & Inn-Donau & $\begin{array}{l}14 \mathrm{~km} \mathrm{~L} . \\
11 \% \mathrm{Br} .\end{array}$ & $73 \mathrm{~m}$ & $18^{\circ} \mathrm{C}$ \\
\hline 26 & Dürrensee . . . . . & $S$ & 1410 & Eisack一Etsch & $\begin{array}{l}500 \mathrm{~m} \mathrm{~L} \\
300 \# \mathrm{Br} .\end{array}$ & seicht & $16^{0} \mathrm{C}$ \\
\hline 27 & Eibsee . . . . & $N$ & 972 & Isar-Donau & $\left\{\begin{array}{lll}2,5 \mathrm{~km} & \mathrm{~L} \\
0,8 & \Rightarrow \mathrm{Br}:\end{array}\right.$ & $28 \mathrm{~m}$ & - \\
\hline 28 & Erlaufsee $\ldots \ldots$ & $\mathrm{N}$ & 835 & Erlaf-Donau & $\left(\begin{array}{lll}1,2 & \mathrm{~km} & \mathrm{~L} . \\
0,6 & , & \mathrm{Br} .\end{array} \mid\right.$ & $44 \mathrm{~m}$ & $16,5^{\circ} \mathrm{C}$ \\
\hline 29 & Faakersee. & S & 560 & Gail-Drau & $\left|\begin{array}{ccc}2,15 & \mathrm{~km} & \mathrm{~L} \\
1,7 & , \mathrm{Br}\end{array}\right|$ & $29,5 \mathrm{~m}$ & - \\
\hline 30 & Faistenauer Hintersee & $\mathrm{N}$ & 685 & Inn-Donau & $\left\{\begin{array}{ccc}1,39 & \mathrm{~km} & \mathrm{~L} . \\
0,72 & , & \mathrm{Br} .\end{array}\right.$ & $22 \mathrm{~m}$ & $\begin{array}{l}14^{0} \mathrm{C} \\
\text { (Juni) }\end{array}$ \\
\hline 31 & Filzsee . . & $\mathrm{Z}$ & 2100 & Enns-Donau & klein & $5-6 \mathrm{~m}$ & $7^{0} \mathrm{C}$ \\
\hline 32 & Hinterer Finstertalersee & $\mathrm{Z}$ & 2259 & Inn-Donau & $\begin{array}{l}300 \mathrm{~m} \mathrm{~L} . \\
200 \Rightarrow \mathrm{Br} .\end{array}$ & - & $9,5^{0} \mathrm{C}$ \\
\hline 33 & Vorderer Finstertalersee. & $\mathrm{Z}$ & 2235 & Inn-Donau & $\begin{array}{l}550 \mathrm{~m} \mathrm{~L} . \\
300 \% \mathrm{Br} .\end{array}$ & - & $10^{\circ} \mathrm{C}$ \\
\hline 34 & Foscagnosee .... & $\mathrm{Z}$ & 2235 & Adda-Po & - & - & - \\
\hline 35 & Fraëlesee . . . . & $\mathrm{Z}$ & 1934 & Adda $-\mathrm{Po}$ & - & 一 & - \\
\hline 36 & Fresenhalssee . . . & $\mathrm{Z}$ & 2150 & Drau & klein & $2-3 \mathrm{~m}$ & - \\
\hline 37 & Fuschelsee . . . & $\mathrm{N}$ & 661 & Traun-Donau & $\left\{\begin{array}{ccc}4 & \mathrm{~km} \mathrm{~L} \\
0,8 & , & \mathrm{Br} .\end{array}\right.$ & $67,3 \mathrm{~m}$ & - \\
\hline 38 & Gafiensee $\ldots .$. & $\mathrm{N}$ & 2312 & Rhein & klein & seicht & $10^{\circ} \mathrm{C}$ \\
\hline 39 & Gaishornsee . . . & $\mathrm{Z}$ & 700 & Enns-Donau & $\left\{\begin{array}{l}700 \mathrm{~m} \mathrm{~L} . \\
250 \% \mathrm{Br} .\end{array}\right.$ & - & - \\
\hline
\end{tabular}




\begin{tabular}{|c|c|c|c|c|c|c|}
\hline $\begin{array}{c}\text { Name des Wasser- } \\
\text { beckens }\end{array}$ & $\stackrel{\Xi}{\circ}$ & $\begin{array}{l}\text { Höhe } \\
\text { iu. } d \text {. } \\
\text { Heere } \\
\text { in } \\
\mathrm{m}\end{array}$ & Flußgebiet & Gröbe & Tiefe & $\begin{array}{c}\text { Ober- } \\
\text { flächen- } \\
\text { wasser- } \\
\text { temperatur } \\
\text { im Sommer }\end{array}$ \\
\hline Gardasee $\cdots$ & $\mathrm{S}$ & 65 & Mincio-Po & $\left\{\begin{array}{lll}55 \mathrm{~km} & \mathrm{~L} \\
18, & \mathrm{Br} .\end{array}\right.$ & $346 \mathrm{~m}$ & $22^{\circ} \mathrm{C}$ \\
\hline Garschinasee. . . . & $\mathrm{N}$ & 2189 & Rhein & $\left\{\begin{array}{l}200 \mathrm{~m} \mathrm{~L} \\
100 \Rightarrow \mathrm{Br}\end{array}\right.$ & $3 \mathrm{~m}$ & $16^{0} \mathrm{C}$ \\
\hline Oberer Giglachsee . . & $\mathrm{Z}$ & 1960 & Enns-Donau & $\left\{\begin{array}{l}390 \mathrm{~m} \mathrm{~L} \\
150 \# \mathrm{Br} .\end{array}\right.$ & - & $10^{\circ} \mathrm{C}$ \\
\hline Vorderer Gosausee . . & $N$ & 908 & Traun-Donau & $\left\{\begin{array}{lll}1,5 & \mathrm{~km} & \mathrm{~L} . \\
0,5 & , & \mathrm{Br} .\end{array}\right.$ & $69,2 \mathrm{~m}$ & - \\
\hline Gösselsdorfersee . . . & $\mathrm{S}$ & 500 & Drau & $\left\{\begin{array}{lll}1,4 & \mathrm{~km} & \mathrm{~L} \\
0,6 & , \mathrm{Br}\end{array}\right.$ & - & - \\
\hline Grundlsee . . . & $\mathrm{N}$ & 709 & Traun-Donau & $\left\{\begin{array}{lll}4,5 & \mathrm{~km} & \mathrm{~L} . \\
1 & \# & \mathrm{Br} .\end{array}\right.$ & $63,8 \mathrm{~m}$ & - \\
\hline Grünsee $\ldots \cdots \cdot$ & $\mathrm{Z}$ & 1699 & Inn-Donau & $\left\{\begin{array}{l}420 \mathrm{~m} \mathrm{~L} . \\
315 \% \mathrm{Br} .\end{array}\right.$ & $32,2 \mathrm{~m}$ & $8^{0} \mathrm{C}$ \\
\hline Haidersee ...... & $\mathrm{Z}$ & 1450 & Etsch & $\left\{\begin{array}{lll}2,34 & \mathrm{~km} & \mathrm{~L} . \\
0,63 & , & \mathrm{Br} .\end{array}\right.$ & $16,5 \mathrm{~m}$ & - \\
\hline Hallstättersee . . & $N$ & 494 & Traun-Donau & $\left\{\begin{array}{lll}8,2 & \mathrm{~km} & \mathrm{~L} . \\
2,1 & , & \mathrm{Br} .\end{array}\right.$ & $125,2 \mathrm{~m}$ & $18,7^{\circ} \mathrm{C}$ \\
\hline Hechtsee $\cdots$ & N & 544 & Inn-Donau & $\left\{\begin{array}{l}750 \mathrm{~m} \mathrm{~L} \\
750, \mathrm{Br} .\end{array}\right.$ & $56,5 \mathrm{~m}$ & - \\
\hline Hintersee bei Ramsau & $\mathrm{N}$ & 732 & Inn-Donau & $\left\{\begin{array}{l}850 \mathrm{~m} \mathrm{~L} . \\
500 \ldots \mathrm{Br}\end{array}\right.$ & - & $18^{\circ} \mathrm{C}$ \\
\hline Hintersteinersee . . & N & 892 & Inn-Donau & $\left\{\begin{array}{lll}1,2 \mathrm{~km} \mathrm{~L} & \\
0,5 \quad, & \mathrm{Br} .\end{array}\right.$ & $35,6 \mathrm{~m}$ & - \\
\hline Hopfensee $\ldots$. & $\mathrm{N}$ & 785 & Lech-Donau & $2,3 \mathrm{~km}$ i. D. & - & - \\
\hline Idrosee $\ldots \ldots$ & $\mathrm{S}$ & 378 & Oglio-Po & $\left\{\begin{array}{l}9,6 \mathrm{~km} \text { L. } \\
1,9, \quad \mathrm{Br} .\end{array}\right.$ & $122 \mathrm{~m}$ & - \\
\hline Iseosee .... & $\mathrm{S}$ & 192 & Oglio-Po & $\left\{\begin{array}{l}15 \mathrm{~km} \mathrm{~L} . \\
4,6, \mathrm{Br} .\end{array}\right.$ & $260 \mathrm{~m}$ & - \\
\hline Jeserzsee . . & S & 620 & Drau & $\left\{\begin{array}{l}550 \mathrm{~m} \mathrm{~L} . \\
280 \% \mathrm{Br} .\end{array}\right.$ & - & 一 \\
\hline Igelsee beim Attersee & $\mathrm{N}$ & 621 & Traun-Donau & $120 \mathrm{~m}$ i. D. & - & - \\
\hline Kalterersee .... & S & 216 & Etsch & $\begin{cases}2 \mathrm{~km} \mathrm{~L} \\
1, & \mathrm{Br}\end{cases}$ & $5-5 \mathrm{~m}$ & $25,7^{\circ} \mathrm{C}$ \\
\hline Kammersee bei Aussee & $\mathrm{N}$ & 720 & Traun-Donau & $\left\{\begin{array}{l}330 \mathrm{~m} \mathrm{~L} . \\
140 \Rightarrow \mathrm{Br} .\end{array}\right.$ & $7 \mathrm{~m}$ & - \\
\hline Kampspitzsee & $\mathrm{Z}$ & 2200 & Enns-Donau & $\left\{\begin{array}{l}225 \mathrm{~m} \mathrm{~L} . \\
110, \mathrm{Br} .\end{array}\right.$ & nicht tief & $1,3^{\circ} \mathrm{C}$ \\
\hline Keutschachersee & S & 508 & Drau & $\left\{\begin{array}{ccc}2 & \mathrm{~km} & \mathrm{~L} \\
1,1 & , & \mathrm{Br}\end{array}\right.$ & $15 \mathrm{~m}$ & - \\
\hline Oberer Klaftersee . & $\mathrm{Z}$ & 2000 & Enns-Donau & $225 \mathrm{~m}$ i. D. & seicht & $7^{0} \mathrm{C}$ \\
\hline Ḱlammjıchsee . & $\mathrm{Z}$ & 2350 & Inn-Donau & $\left\{\begin{aligned} 100 \mathrm{~m} \mathrm{~L} & \\
60 & \cdots \mathrm{Br} .\end{aligned}\right.$ & seicht & $17,6^{\circ} \mathrm{C}$ \\
\hline
\end{tabular}





\begin{tabular}{|c|c|c|c|c|c|c|c|c|c|c|c|c|c|c|c|}
\hline Nr. & $\begin{array}{l}\text { Name des Wasser- } \\
\text { beckens }\end{array}$ & 气ू & $\mid \begin{array}{c}\text { Höhe } \\
\text { ï. } d . \\
\text { Meere } \\
\text { in } \\
\mathrm{m}\end{array}$ & FluBgebiet & Gröbe & Tiefe & 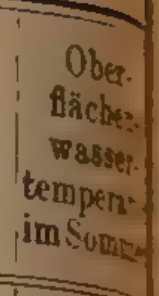 & ir. & $\begin{array}{l}\text { Name des Wasser- } \\
\text { beckens }\end{array}$ & 总 & $\begin{array}{l}\text { Höhe } \\
\text { ü. d. } \\
\text { IIeere } \\
\text { in } \\
\text { m }\end{array}$ & FluBgebiet & Gröbe & Tiefe & $\begin{array}{l}\text { Ober- } \\
\text { flächen- } \\
\text { wasser- } \\
\text { temperatur } \\
\text { im Sommer }\end{array}$ \\
\hline 13 & Badersee & $x$ & 830 & Isar-Donaus & $200 \mathrm{~m}$ i. D. & $15 \mathrm{~m}$ & $10,5^{\circ}:$ & $\sharp 1$ & & s & 65 & & $\begin{array}{l}55 \mathrm{~km} \mathrm{~L} \text {. } \\
18 \ldots \mathrm{Br} \text {. }\end{array}$ & $346 \mathrm{~m}$ & $2200 \mathrm{C}$ \\
\hline 14 & Bannwaldsee & $x$ & 792 & Lech-Donau & $\left\{\begin{array}{lll}2 & \mathrm{~km} & \mathrm{~L} \\
1 & \Rightarrow & \mathrm{Br} \\
\text { sehr } & \text { klein }\end{array}\right.$ & $\begin{array}{l}11 \mathrm{~m} \\
\text { seicht }\end{array}$ & - & 41 1 & Garsthinasce. . . . & $x$ & 2189 & Rhein & $200 \mathrm{~m} \mathrm{~L}$. & $3 \mathrm{~m}$ & $10^{\circ} \mathrm{C}$ \\
\hline 15) & $\begin{array}{l}\text { Berghaustümpelsee . } \\
\text { Berglsteinersee. . . }\end{array}$ & $\begin{array}{l}7 \\
3\end{array}$ & $\begin{array}{r}1700 \\
740\end{array}$ & $\begin{array}{l}\text { Lieser-Drau } \\
\text { Inn-Donau }\end{array}$ & $\left\{\begin{array}{l}\text { sehr klein } \\
300 \mathrm{~m} \mathrm{~L} . \\
200, \mathrm{Br} .\end{array}\right.$ & $1 \mathrm{~m}$ & - & 2 & Oberer Giglachsee . & $\mathrm{z}$ & 1960 & Enns-Donau & $\begin{array}{l}390 \mathrm{~m} \mathrm{~L} \\
150, \mathrm{Br}\end{array}$ & - & $10^{\circ} \mathrm{C}$ \\
\hline 17 & Bodensee .... & $x$ & 398 & Rhein & $\left\{\begin{array}{lll}69 & \mathrm{~km} & \mathrm{~L} \\
13 & \mathrm{Br}\end{array}\right.$ & $308 \mathrm{~m}$ & $17,80 \mathrm{r}$ & 13 & $\begin{array}{l}\text { Gorderer Gosausee . } \\
\text { Gösselsdorfersee . . }\end{array}$ & $\begin{array}{l}x \\
s\end{array}$ & $\begin{array}{l}308 \\
500\end{array}$ & $\begin{array}{c}\text { Traun-Donau } \\
\text { Drau }\end{array}$ & $\left\{\begin{array}{lll}1,5 & \mathrm{~km} & \mathrm{~L} . \\
0,5 & \mathrm{Br}\end{array} \mid\right.$ & $69,2 \mathrm{~m}$ & - \\
\hline $\begin{array}{l}18 \\
19\end{array}$ & $\begin{array}{l}\text { Grober Büdensee } \\
\text { Kleiner Büdensee }\end{array}$ & $\begin{array}{l}s \\
s\end{array}$ & $\begin{array}{l}2300 \\
2300\end{array}$ & $\begin{array}{l}\text { Drau } \\
\text { Drau }\end{array}$ & klein & $\begin{array}{l}\text { seicht } \\
\text { seicht }\end{array}$ & $\begin{array}{l}16,20 \text { 个 } \\
18,0 \mathrm{c}\end{array}$ & 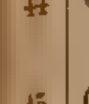 & Grundlsee . . . . & $\mathrm{N}$ & & Traun-Donan & $\mid \begin{array}{llll}0,6 & & \\
4 & 4,5 & \mathrm{~km} & \mathrm{Lr}\end{array}$ & - & - \\
\hline 20 & Boësee ..... & $S$ & 2282 & $\begin{array}{c}\text { Piave } \\
\text { Tieser-Drau }\end{array}$ & $\begin{array}{c}\text { ca. } 100 \mathrm{mi} \text {. D. } \\
1,5 \mathrm{~km} \mathrm{~L} .\end{array}$ & tief & $\begin{array}{c}10,50 \mathrm{r} \\
240 \mathrm{r}\end{array}$ & 5 & (yrumise & 10 & & Haul-Donall & $\left|\begin{array}{lll}1 & \text { Br. }\end{array}\right|$ & $63,8 \mathrm{~m}$ & - \\
\hline $\begin{array}{l}21 \\
0,\end{array}$ & Brennsee . . & $\begin{array}{l}Z \\
\mathrm{~s}\end{array}$ & $\begin{array}{l}742 \\
449\end{array}$ & $\begin{array}{l}\text { Lieser-Drau } \\
\text { Brenta }\end{array}$ & $\left\{\begin{array}{l}1,5 \mathrm{~km} \mathrm{~L} \\
4,2 \mathrm{~km} \mathrm{~L} \\
17\end{array}\right.$ & $49 \mathrm{~m}$ & & 46 & Grünsee $\cdot$ & $\mathrm{Z}$ & 1699 & Inn-Donau & $\left\{\begin{array}{lll}420 & \mathrm{~m} & \mathrm{~L} \\
315 & \ldots \mathrm{Br}\end{array} \mid\right.$ & $32,2 \mathrm{~m}$ & 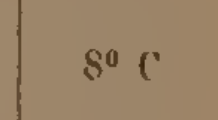 \\
\hline 23 & Cavazzose" & is & 195 & Tagliamento & 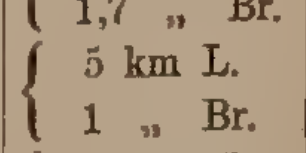 & $41 \mathrm{~m}$ & & 4 & Haidersee . . & 2 & 1450 & Etsch & $\left\{\begin{array}{lll}2,34 & \mathrm{~km} & \mathrm{~L} . \\
0,63 & \mathrm{Br} . & \mathrm{Br} \\
8,2 \mathrm{~km} & \mathrm{~L} .\end{array} \mid\right.$ & $16,5 \mathrm{~m}$ & - \\
\hline 24 & | Ceisee & $\mathrm{s}$ & 927 & Ceibach-Etsch & $\left\{\begin{array}{l}400 \mathrm{~m} \mathrm{~L} . \\
150 \Rightarrow \mathrm{Br} .\end{array}\right.$ & $6,5 \mathrm{~m}$ & - & 49 & $\begin{array}{l}\text { Hallstattersee . } \\
\text { Hechtsee . . . }\end{array}$ & $\mathrm{N}$ & 544 & Inn-Donau & $\left\{\begin{array}{lll}2,1 & 11 & \mathrm{Br} \\
750 & \mathrm{~m} & \mathrm{~L}\end{array}\right.$ & $\begin{array}{r}125,2 \mathrm{~m} \\
56.5 \mathrm{~m}\end{array}$ & $18,7^{\circ} \mathrm{C}$ \\
\hline $2 \%$ & Chiemsec & $\lambda$ & 519 & $\begin{array}{l}\text { Inn-Donau } \\
\text { Eisack-Etsch }\end{array}$ & 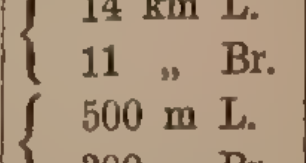 & $\begin{array}{l}73 \mathrm{~m} \\
\text { seicht }\end{array}$ & $18^{\circ} \mathrm{C}$ & 50 & Hinterseo bei Ramsau & $\mathrm{N}$ & 732 & Inn-Donau & $\left\{\begin{array}{lll}850 & \text { B } & \mathrm{Br} \\
500 & \mathrm{~m} & \mathrm{Br} \\
5 r_{0}\end{array}\right.$ & - & $18^{10} \mathrm{C}$ \\
\hline 27 & $\begin{array}{l}\text { Dürrensee } \\
\text { Eilbsee . }\end{array}$ & $x$ & $\begin{array}{r}1410 \\
972\end{array}$ & $\begin{array}{l}\text { Eisack-Etsch } \\
\text { Isar-Donau }\end{array}$ & $\left\{\begin{array}{l}300, \mathrm{Br} . \\
2,5 \mathrm{~km} \\
0,8, \mathrm{Lr} \\
\Rightarrow\end{array}\right.$ & $28 \mathrm{~m}$ & - & 51 & $\begin{array}{l}\text { Hintersteinersee . . } \\
\text { Hopfensee . . . . }\end{array}$ & $\begin{array}{l}N \\
N\end{array}$ & $\begin{array}{l}892 \\
785\end{array}$ & $\begin{array}{l}\text { Inn-Donau } \\
\text { Lech-Donau }\end{array}$ & 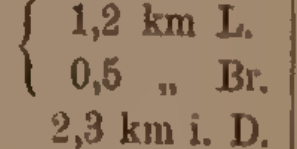 & $\begin{array}{c}35,6 \mathrm{~m} \\
-\end{array}$ & - \\
\hline 28 & Erlaufsee & $\mathrm{N}$ & 835 & Erlafi-Donau & $\left\{\begin{array}{lll}1,2 \mathrm{~km} & \mathrm{~L} \\
0,6 & \mathrm{Br}\end{array}\right.$ & $44 \mathrm{~m}$ & $16,0^{\circ} \mathrm{C}$ & 53 & Idrosee & S & 378 & Oglio-Po & $\left\{\begin{array}{lll}9,6 & \mathrm{~km} & \mathrm{~L} \\
1,9 & , & \mathrm{Br} .\end{array} \mid\right.$ & $122 \mathrm{~m}$ & - \\
\hline 29 & Faakersee & $\mathrm{s}$ & 5601 & Gail-Drau & $\left\{\begin{array}{ccc}2,15 & \mathrm{~km} & \mathrm{~L} \\
1,7 & , \mathrm{Br}\end{array} \mid\right.$ & $29,5 \mathrm{n}$ & - & 5 & Iseosee & $\mathrm{s}$ & 192 & Oglio-Po & 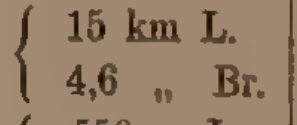 & $260 \mathrm{~m}$ & $\cdots$ \\
\hline 30 & Faistenauer Hintersee & $x$ & 685 & Inn-Donau & $\left\{\begin{array}{lll}1,39 & \mathrm{~km} & \mathrm{~L} . \\
0,72, . & \mathrm{Br} .\end{array}\right.$ & $22 \mathrm{~m}$ & $\begin{array}{l}14^{\circ} \mathrm{C} \\
\text { (Juni) }\end{array}$ & 50 & Jeserzsee $\ldots \ldots$ & $S$ & 620 & Drau & $\left\{\begin{array}{lll}550 & \mathrm{~m} & \mathrm{~L} \\
280 & \cdots \mathrm{Br}\end{array} \mid\right.$ & - & \\
\hline 31 & Filzsec & $\mathrm{Z}$ & 2100 & Enns--Donau & klein & $5-6 \mathrm{~m}$ & $7^{\circ} \mathrm{C}$ & 56 & Igelsee beim Attersee & $\mathrm{N}$ & 621 & Traun-Donau & $120 \mathrm{~m} \mathrm{i}^{\circ} \mathrm{D}$. & - & - \\
\hline 3: & Hinterer Finstertalersee & $\mathrm{Z}$ & 2259 & Inn-Donau & $\begin{cases}300 \mathrm{~m} \mathrm{~L} & \\
200 & \mathrm{Br} .\end{cases}$ & 一 & $9,5^{\circ} \mathrm{C}$ & $5 i$ & Kalterersee . & $\mathrm{S}$ & 216 & Etsch & $\left\{\begin{array}{l}2 \mathrm{~km} \mathrm{I} \\
1,3 \mathrm{Br}\end{array}\right.$ & $5-3 \mathrm{~m}$ & $25,7^{0} \mathrm{C}$ \\
\hline 33 & Vordorer Finstertalersee. & Z & 2235 & Inn-Donau & $\begin{cases}550 & \mathrm{~m} \mathrm{~L} \\
300 & \cdots \mathrm{Br} .\end{cases}$ & - & $10^{\circ} \mathrm{C}$ & 38 & Kammersee bei Aussee & $\mathrm{N}$ & 720 & Traun-Donau & $\left\{\begin{array}{l}330 \mathrm{~m} \mathrm{~L} \\
140 \ldots \mathrm{Br} .\end{array}\right.$ & $7 \mathrm{~m}$ & - \\
\hline 34 & $\begin{array}{l}\text { Foscagnosee. } \\
\text { Fraëlesee . . }\end{array}$ & $\begin{array}{l}Z \\
Z\end{array}$ & $\begin{array}{l}2235 \\
1934\end{array}$ & $\begin{array}{l}\text { Adda-Po } \\
\text { Adda-Po }\end{array}$ & $=$ & - & - & 59 & Kampspitzsee & $\mathrm{Z}$ & 2200 & Enns-Donau & $\begin{cases}225 \mathrm{~m} & \mathrm{~L} \\
110, & \mathrm{Br} .\end{cases}$ & nicht tiof & $1,3^{\circ} \mathrm{C}$ \\
\hline 36 & Fresenhalssec & $\mathrm{L}$ & 2150 & Drau & $\int \quad 4 \mathrm{~km} \mathrm{~L}$ & $2-3 \mathrm{~m}$ & - & 601 & Keutschachersee. & $\mathrm{s}$ & 508 & Drau & $\left\{\begin{array}{rl}2 \mathrm{~km} & \mathrm{~L} \\
1,1 & \cdots \\
\mathrm{Br}\end{array}\right.$ & $15 \mathrm{~m}$ & - \\
\hline $3 x$ & $\begin{array}{l}\text { Fuschelsee } \\
\text { Gafiensee }\end{array}$ & $\begin{array}{l}\kappa \\
\kappa\end{array}$ & $\begin{array}{r}661 \\
2312\end{array}$ & $\begin{array}{l}\text { Traun-Donau } \\
\text { Rhein }\end{array}$ & $\int_{\text {klein }}^{0,8} \mathrm{Br}$. & $\begin{array}{l}67,3 \mathrm{~m} \\
\text { seicht }\end{array}$ & $10^{\circ} \mathrm{C}$ & 61 & Oberer Kiaftersee . & Z & 2000 & Enns-Donau & $225 \mathrm{~m} \mathrm{i} . \mathrm{D}$. & seicht & $7^{\circ} \mathrm{C}$ \\
\hline $3 !$ & Gaishornsee & 7. & 700 & Enns-Donau & $\left\{\begin{array}{l}700 \mathrm{~m} \mathrm{~L} . \\
250 \Rightarrow \mathrm{Br}\end{array}\right.$ & - & -1 & & Mammjuchsee . . . & $\mathrm{Z}$ & 2350 & Inn-Donau & $60 \# \mathrm{Br}$. & seicht & $17,6^{\circ} \mathrm{C}$ \\
\hline
\end{tabular}




\begin{tabular}{|c|c|c|c|c|c|c|c|}
\hline Nr. & $\begin{array}{c}\text { Name des Wasser- } \\
\text { beckens }\end{array}$ & ัํํ & $\begin{array}{l}\text { Höhe } \\
\text { i. d. } \\
\text { Meere } \\
\text { in } \\
\mathrm{m}\end{array}$ & FluBgebiet & Größe & Tiefe & $\begin{array}{c}\text { Ober } \\
\text { fläche] } \\
\text { wasse } \\
\text { tempera } \\
\text { im Somr }\end{array}$ \\
\hline 63 & Mittlerer Klippensee . & $\mathrm{Z}$ & 2257 & Enns-Donau & $100 \mathrm{~m}$ i. $\mathrm{D}$. & $3-4 \mathrm{~m}$ & $5^{0} \mathrm{C}$ \\
\hline 64 & Klopeinersee . . . & $\mathrm{S}$ & 448 & Drau & $\left\{\begin{array}{lll}2,1 & \mathrm{~km} & \mathrm{~L} . \\
0,8 & , & \mathrm{Br} .\end{array}\right.$ & $48 \mathrm{~m}$ & - \\
\hline 65 & Königssee ..... & $\mathrm{N}$ & 603 & Inn-Donau & $\left\{\begin{array}{l}8 \mathrm{~km} \mathrm{~L} \\
2, \mathrm{Br}\end{array}\right.$ & $190 \mathrm{~m}$ & $\begin{array}{l}12,2^{0} \\
\text { (Sept. }\end{array}$ \\
\hline 66 & Königsangersee. . . . & $\mathrm{Z}$ & 2173 & Lieser-Drau & $\left\{\begin{array}{r}200 \mathrm{~m} \mathrm{~L} . \\
50, \mathrm{Br} .\end{array}\right.$ & seicht & - \\
\hline 67 & Kreuthsee . . . . . & $\mathrm{N}$ & 800 & Inn-Donau & klein & - & - \\
\hline 68 & Kreuzjochsee ... & S & 2200 & Eisack-Etsch & $\left\{\begin{array}{rll}225 & \mathrm{~m} \mathrm{~L} . \\
75 & . . & \mathrm{Br} .\end{array}\right.$ & seicht & $9,7^{\circ} \mathrm{C}$ \\
\hline 69 & Kröllensee . . . . & $\mathrm{N}$ & ? 1500 & Traun-Donau & sehr klein & seicht & - \\
\hline 70 & Krottensee .... & $\mathrm{N}$ & 590 & Traun-Donau & $390 \mathrm{~m}$ i. D. & $45,5 \mathrm{~m}$ & 一 \\
\hline 71 & Krummsee. . . & $\mathrm{N}$ & 557 & Inn-Donau & $\left\{\begin{array}{l}350 \mathrm{~m} \mathrm{~L} . \\
150 \% \mathrm{Br} .\end{array}\right.$ & $2,5 \mathrm{~m}$ & $23^{\circ} \mathrm{C}$ \\
\hline 72 & Lagoraisee . . . & $\mathrm{S}$ & 1868 & Avisio-Etsch & $\left\{\begin{array}{c}700 \mathrm{~m} \mathrm{~L} . \\
250 \% \mathrm{Br} .\end{array}\right.$ & über $25 \mathrm{~m}$ & - \\
\hline 73 & Hinterer Lahngangsee & $\mathrm{N}$ & 1560 & Traun-Donau & $\left\{\begin{array}{l}400 \mathrm{~m} \mathrm{~L} . \\
200 \% \mathrm{Br} .\end{array}\right.$ & $19,91 \mathrm{~m}$ & - \\
\hline 74 & Vorderer Lahngangsee & $\mathrm{N}$ & 1555 & Traun-Donau & $\left\{\begin{array}{l}800 \mathrm{~m} \mathrm{~L} \\
400, \mathrm{Br} .\end{array}\right.$ & $77 \mathrm{~m}$ & - \\
\hline 75 & Lambrechtersee . . & $\mathrm{Z}$ & 1050 & Mur-Drau & $200 \mathrm{~m}$ i. D. & - & - \\
\hline 76 & Vorderer Langbathsee & $\mathrm{N}$ & 675 & Traun-Donau & $\left\{\begin{array}{lll}1,1 & \mathrm{~km} & \mathrm{~L} . \\
0,3 & , & \mathrm{Br} .\end{array}\right.$ & $33,8 \mathrm{~m}$ & - \\
\hline 77 & Oberer Lanischsee & $\mathrm{Z}$ & 2300 & Lieser-Drau & sehr klein & - & $\begin{array}{l}\text { Schmel: } \\
\text { wasserst }\end{array}$ \\
\hline 78 & Unterer Lanischsee . & $\mathrm{Z}$ & 2200 & Lieser-Drau & sehr klein & - & - \\
\hline 79 & Lansersee . . . . & $\mathrm{Z}$ & 851 & Inn-Donau & $200 \mathrm{~m}$ i. $\mathrm{D}$. & - & $29^{\circ} \mathrm{C}$ \\
\hline 80 & Lansermoorsee. . . . & $\mathrm{Z}$ & 800 & Inn-Donau & $100 \mathrm{~m}$ i. D. & sehr seicht & - \\
\hline 81 & Lasessee . . . & S & 639 & Avisio-Etsch & $\left\{\begin{array}{lll}700 & \mathrm{~m} & \mathrm{~L} \\
225 & , \mathrm{Br} .\end{array}\right.$ & $31 \mathrm{~m}$ & $23^{\circ} \mathrm{C}$ \\
\hline 82 & Lauterersee . . & $\mathrm{Z}$ & 2400 & Inn--Donau & sehr klein & $4-8 \mathrm{~m}$ & $5^{0} \mathrm{C}$ \\
\hline 83 & Lavaronesee . . . . . & $\mathrm{S}$ & 1100 & Astico-Brenta & $\begin{cases}350 \mathrm{~m} \mathrm{~L} & \\
225 & \Rightarrow \mathrm{Br}\end{cases}$ & $15,8 \mathrm{~m}$ & - \\
\hline 84 & Ledrosee . . . & S & 668 & Mincio-Po & $\left\{\begin{array}{ccc}2,8 & \mathrm{~km} & \mathrm{~L} . \\
1,4 & \therefore & \mathrm{Br} .\end{array}\right.$ & ? $47,6 \mathrm{~m}$ & - \\
\hline 85 & Leonhardsee . & $\mathrm{Z}$ & 530 & Drau & sehr klein & seicht & 一 \\
\hline 86 & Leopoldsteinersee : & $\mathrm{N}$ & 619 & Enns-Donau & $\left\{\begin{array}{lll}1,4 & \mathrm{~km} & \mathrm{~L} \\
0,5 & , & \mathrm{Br}\end{array}\right.$ & $32 \mathrm{~m}$ & $17^{\circ} \mathrm{C}$ \\
\hline 87 & Levicosee ... & $S$ & 449 & Brenta & $\left\{\begin{array}{lll}2,48 & \mathrm{~km} & \mathrm{~L} . \\
0,68 & , & \mathrm{Br} .\end{array}\right.$ & $35 \mathrm{~m}$ & - \\
\hline 88 & Lichtsee ..... & $\mathrm{Z}$ & 2100 & Inn-Donau & $\left\{\begin{array}{l}200 \mathrm{~m} \mathrm{~L} \\
150 \ldots \mathrm{Br}\end{array}\right.$ & wenige $\mathrm{m}$ & $9^{0} \mathrm{C}$ \\
\hline
\end{tabular}




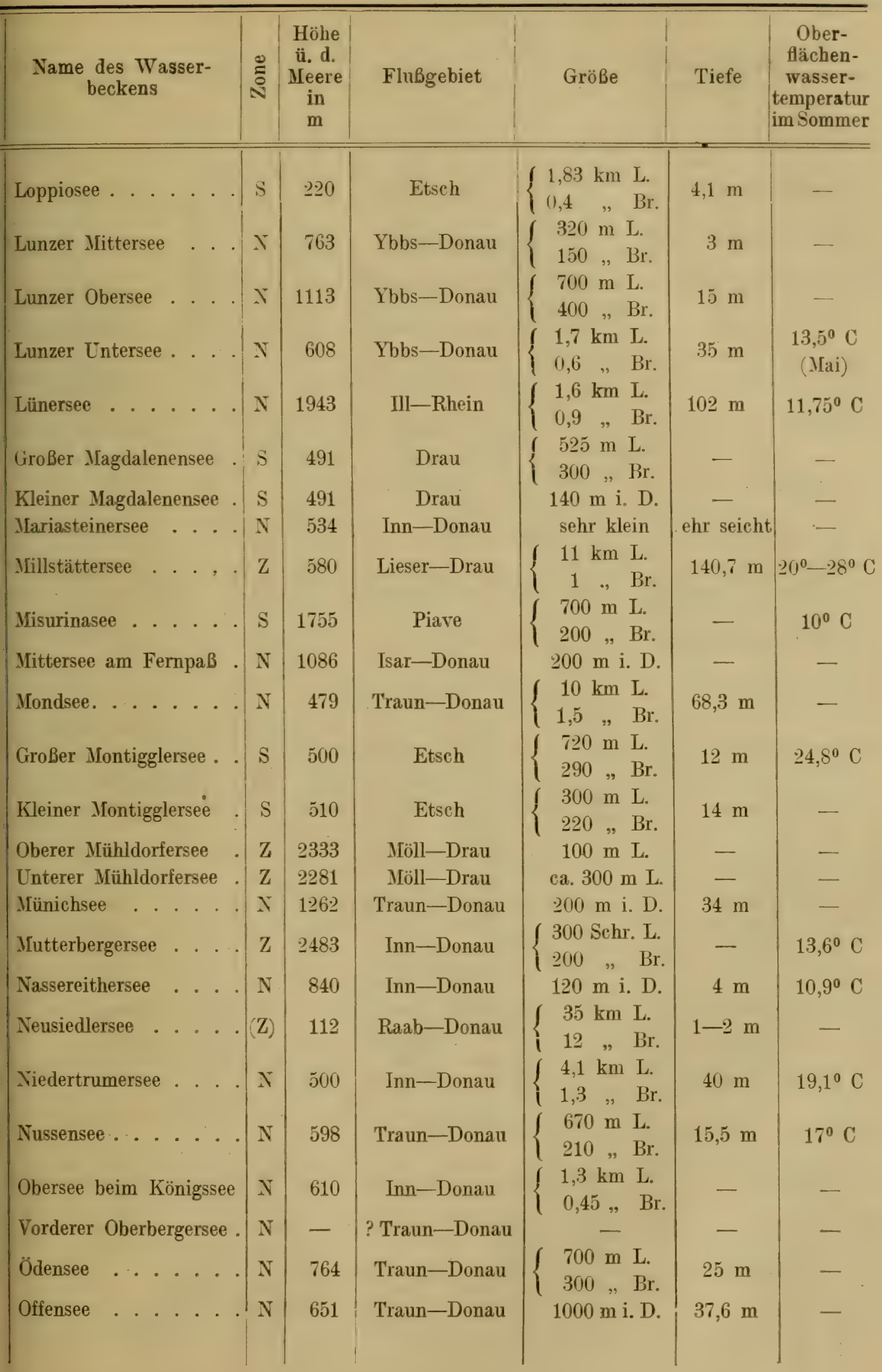





\begin{tabular}{|c|c|c|c|c|c|c|c|c|c|c|c|c|c|c|c|}
\hline Ir. & $\begin{array}{c}\text { Name des Wasser- } \\
\text { beckens }\end{array}$ & : & $\begin{array}{c}\text { Hiihe } \\
\text { ii. d. } \\
\text { Jieere } \\
\text { in } \\
m\end{array}$ & FluBgebiet & Gröbe & Tiefe & $\begin{array}{l}\text { Ober. } \\
\text { fächer. } \\
\text { wasser. } \\
\text { temperar. } \\
\text { im Sommes. }\end{array}$ & St & $\begin{array}{c}\text { Yame des Wasser- } \\
\text { beckens }\end{array}$ & : & \begin{tabular}{|c|} 
Hölue \\
â. d. \\
Meere \\
in \\
$m$
\end{tabular} & Flubgebiet & Gröbe & Tiefe & $\begin{array}{l}\text { ()her- } \\
\text { tllichen- } \\
\text { wasser- } \\
\text { temperatur } \\
\text { im Sommer }\end{array}$ \\
\hline 63.3 & Mittlerer Klippensee & $L$ & 2.257 & Enns-Donau & $100 \mathrm{~m}$ i. $\mathrm{D}$. & $3-4 \mathrm{ml}$ & $5^{0} \mathrm{C}$ & $4: 4$ & Loppiosee . . . : & $S$ & 220 & Etsch & $\begin{array}{lll}1,83 & \mathrm{~km} & \mathrm{~L} \\
0,4 & \mathrm{Br} .\end{array} \mid$ & $1,1 \mathrm{~m}$ & - \\
\hline iit & Klopeinersee & $S$ & 448 & Drau & $\left\{\begin{array}{l}2,1 \mathrm{~km} \mathrm{~L} \\
0,8, \quad \mathrm{Br} . \\
8 \mathrm{~km} \mathrm{~L} .\end{array}\right.$ & $48 \mathrm{~m}$ & (1) & 91 & Lunzer Mittersee . . & $\mathrm{N}$ & 763 & Ybbs--Donau & $\left\{\begin{array}{l}320 \mathrm{~m} \mathrm{~L} \\
150, \mathrm{Br}\end{array}\right.$ & $3 \mathrm{~m}$ & - \\
\hline bij & Künigssee .... & $x$ & 6013 & Inn-Donau & $\left\{\begin{array}{l}2 \% \mathrm{Br} \\
200 \mathrm{~m} \mathrm{~L}\end{array}\right.$ & $190 \mathrm{~m}$ & $\begin{array}{l}12,20 \text { ! } \\
\text { (Septt. }\end{array}$ & (4) ${ }^{1}$ & Lunzer Obersee ... & $x$ & 1113 & Ybbs-Donau & $\left\{\begin{array}{l}700 \mathrm{~m} \mathrm{I} \\
400=\mathrm{Br} .\end{array}\right.$ & $15 \mathrm{~m}$ & - \\
\hline bif & $\begin{array}{l}\text { Königsangersee. . . } \\
\text { Kreuthice . . . . . }\end{array}$ & $x$ & $\begin{array}{r}2173 \\
800\end{array}$ & $\begin{array}{l}\text { Lieser-Drau } \\
\text { Inn-Donau }\end{array}$ & $\left\{\begin{array}{c}50, \mathrm{Br} . \\
\text { klein }\end{array}\right.$ & $\begin{array}{l}\text { seicht } \\
-\end{array}$ & & ? & Lunzer Untersee .... & $x$ & 608 & Ybbs--Donau & $\left\{\begin{array}{lll}1,7 \mathrm{~km} \mathrm{~L} & \mathrm{~K} \\
0,6 & , \mathrm{Br} .\end{array} \mid\right.$ & $95 \mathrm{~m}$ & $\begin{array}{l}13,5^{\circ} \mathrm{C} \\
\text { (Mtai) }\end{array}$ \\
\hline dis & Kreuzjochsee & $s$ & 20100 & Eisack-Etsch & $\left\{\begin{array}{ccc}225 & \mathrm{~m} & \mathrm{~L} \\
75 & . & \mathrm{Br}\end{array}\right.$ & seicht & $9, \pi^{\circ} \mathrm{C}$ & 3 & Lünersee $\cdots \cdots$ & $N$ & 1943 & 11-Rhein & $\left\{\begin{array}{lll}1,6 & \mathrm{~km} & \mathrm{~L}_{\mathrm{s}} \\
0,9 & \mathrm{Br}\end{array}\right.$ & $102 \mathrm{~m}$ & $11,75^{\circ} \mathrm{C}$ \\
\hline $6 ;$ & Kröllensee ... & $x$ & $\because 1500$ & Traun-Donau & $\begin{array}{l}\text { sehr klein } \\
390 \mathrm{~m}\end{array}$ & seicht & - & 14 & GroBer Magdalenensee & $\mathrm{s}$ & 491 & Drau & $\left\{\begin{array}{l}525 \mathrm{~m} \mathrm{~L} \\
300 \ldots \mathrm{Br}\end{array}\right.$ & - & - \\
\hline 71! & Krottensee . . . & $x$ & 590 & Traun-Donau & $\int \begin{array}{c}390 \mathrm{~m} \mathrm{i} . \mathrm{D} \\
\int 50 \mathrm{~m} \mathrm{~L} .\end{array}$ & $\mp 0,0 \mathrm{II}$ & 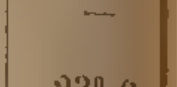 & 消 & $\begin{array}{l}\text { Keiner Magdalenensee } \\
\text { Variasteinersee }\end{array}$ & $\begin{array}{l}S \\
\mathrm{y}\end{array}$ & 491 & Drau & $140 \mathrm{~m} \mathrm{i.} \mathrm{D}$ & - & - \\
\hline i1 & $\begin{array}{l}\text { Krummsec. . . . } \\
\text { Lagoraiseo . . . }\end{array}$ & $s$ & 1468 & $\begin{array}{l}\text { Inn-Donau } \\
\text { Avisio-Etsch }\end{array}$ & $\left\{\begin{array}{l}150, \mathrm{Br} . \\
700 \mathrm{~m} \\
250\end{array}\right.$ & aiber $25 \mathrm{~m}$ & $23^{n} \mathrm{c}$ & $9 \bar{i}$ & Ifillstättersee $\ldots$ & $\mathrm{Z}$ & 580 & Liesor-Drau & $\left\{\begin{array}{c}11 \mathrm{~km} \mathrm{~L} . \\
1 \ldots \mathrm{Br} .\end{array}\right.$ & $140,7 \mathrm{~m}$ & $20^{\circ}-28^{\circ} \mathrm{C}$ \\
\hline 73 & Hinterer Lahngangsee & $x$ & 1560 & Traun-Donau & $\left\{\begin{array}{l}400 \mathrm{~m} \mathrm{~L} . \\
200 \Rightarrow \mathrm{Br} . \\
800 \mathrm{~m} \mathrm{~L} .\end{array}\right.$ & $19,91 \mathrm{~m}$ & - & 93 & $\begin{array}{l}\text { Yisurinasee .... } \\
\text { Mittersee am Fermpa B }\end{array}$ & S & $\begin{array}{l}1755 \\
1086\end{array}$ & $\begin{array}{c}\text { Piave } \\
\text { Isar-Donau }\end{array}$ & $\left\{\begin{array}{c}700 \mathrm{~m} \mathrm{~L} \\
200 \% \mathrm{Br} \\
200 \mathrm{~m} \mathrm{i.} \mathrm{D} .\end{array}\right.$ & - & $\begin{array}{c}10^{\circ} \mathrm{C} \\
-\end{array}$ \\
\hline it & Vorderer Lahngangsee & $x$ & $155 \%$ & Traun-Donau & 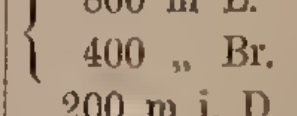 & $77 \mathrm{~m}$ & - & $1+10$ & Mondsee. & $\mathrm{N}$ & 479 & Traun-Donau & $\left\{\begin{array}{c}10 \mathrm{~km} \mathrm{~L} . \\
1,5 \ldots \mathrm{Br} .\end{array}\right.$ & $68,3 \mathrm{~m}$ & - \\
\hline 7i) & $\begin{array}{l}\text { Lambrechtersee } \\
\text { Vorderer Langbathsee }\end{array}$ & $\begin{array}{l}Z \\
x\end{array}$ & $\begin{array}{r}1050 \\
675\end{array}$ & $\begin{array}{c}\text { Mur-Drau } \\
\text { Traun-Donau }\end{array}$ & $\left\{\begin{array}{l}200 \mathrm{~m} \mathrm{i} . \mathrm{D} . \\
1,1 \mathrm{~km} \mathrm{~L} . \\
0,3 \quad \mathrm{Br} .\end{array}\right.$ & $33,8 \mathrm{~m}$ & - & 101 & Großer Montigglersee. & $\mathbf{S}$ & 500 & Etsch & $\left\{\begin{array}{l}720 \mathrm{~mL} \\
290 \mathrm{Lr} .\end{array}\right.$ & $12 \mathrm{~m}$ & $24,8^{\circ} \mathrm{C}$ \\
\hline 77 & Oberer Liniselsee . . & $z$ & 2300 & Lieser-Drau & sehr klein & - & Schmels. & $10 \mathrm{E}$ & Kleiner Montigglersee & $\mathbf{S}$ & 510 & Etsch & $\left\{\begin{array}{l}300 \mathrm{~m} \mathrm{~L} \\
220 \text { " Br. }\end{array}\right.$ & $14 \mathbf{m}$ & - \\
\hline 78 & I'nterer Lanischsee & $\mathrm{Z}$ & 2200 & Lieser-Drau & sehr klein & - & $\begin{array}{c}\text { wasseret } \\
-\end{array}$ & $\begin{array}{l}1113 \\
1114\end{array}$ & $\begin{array}{l}\text { Oberer Mühldorfersee } \\
\text { Unterer Mühldorfersee }\end{array}$ & $\begin{array}{l}\mathrm{Z} \\
\mathrm{Z}\end{array}$ & $\begin{array}{l}2333 \\
2281\end{array}$ & $\begin{array}{l}\text { Möll-Drau } \\
\text { Möll-Drau }\end{array}$ & $\begin{array}{c}100 \mathrm{~m} \mathrm{~L} \\
\mathrm{ca}, 300 \mathrm{~m} \mathrm{~L} .\end{array}$ & - & - \\
\hline $\begin{array}{l}8 ! \\
80\end{array}$ & $\begin{array}{l}\text { Lansersere . . . } \\
\text { Lannermoorsee. . }\end{array}$ & $\begin{array}{l}\mathrm{Z} \\
\mathrm{Z}\end{array}$ & $\begin{array}{l}851 \\
800\end{array}$ & $\begin{array}{l}\text { Inn-Donau } \\
\text { Inn-Donau }\end{array}$ & $\begin{array}{l}200 \mathrm{~m} \mathrm{i.} \mathrm{D.} \\
100 \mathrm{~m} \mathrm{i.} \mathrm{D.}\end{array}$ & sehr seicl & $29^{\circ} \mathrm{C}$ & & Jünichsee $\cdots$ & $\mathbf{N}$ & 1262 & Traun-Donau & $200 \mathrm{~m}$ i. $\mathrm{D}$ & $34 \mathrm{~m}$ & - \\
\hline 81 & Lalsissece . . & $\mathrm{S}$ & 639 & Avisio-Etsch & $\left\{\begin{array}{l}700 \mathrm{~m} \mathrm{~L} . \\
225, \mathrm{Br}\end{array}\right.$ & $31 \mathrm{~m}$ & $23^{\circ} \mathrm{C}$ & & Nutterbergersee . . & $\mathrm{Z}$ & 2483 & Inn-Donau & $\left\{\begin{array}{l}300 \text { Schr. I } \\
200 \text { is Br. }\end{array}\right.$ & & $13,6^{\circ} \mathrm{C}$ \\
\hline $\begin{array}{l}8: 2 \\
83\end{array}$ & $\begin{array}{l}\text { Lanterersed . . } \\
\text { Lalvaroneser. . . }\end{array}$ & $\begin{array}{l}Z \\
s\end{array}$ & $\begin{array}{l}2400 \\
1100\end{array}$ & $\begin{array}{c}\text { Inn-Donau } \\
\text { Astico-Brenta }\end{array}$ & $\left\{\begin{array}{l}\text { sehr klein } \\
350 \mathrm{~m} \mathrm{~L}\end{array}\right.$ & $\begin{array}{l}4-8 \mathrm{~m} \\
15,8 \mathrm{~m}\end{array}$ & $\begin{array}{l}5^{\circ} \mathrm{C} \\
-\end{array}$ & 1118 & Neusiedlersee $\ldots$ & $(\mathbf{Z})$ & 112 & Rstab-Donau & $\left\{\begin{array}{l}120 \mathrm{mi}, \mathrm{D} . \\
35 \mathrm{~km} \mathrm{~L} . \\
12, \mathrm{lir} .\end{array}\right.$ & $\begin{array}{c}4 \mathrm{~m} \\
1-2 \mathrm{~m}\end{array}$ & - \\
\hline 84 & Jedrosee . . & $s$ & 668 & Vincio-Po & $\left\{\begin{array}{lll}2,8 \mathrm{~km} & \mathrm{~L} . \\
1,4 & , & \mathrm{Br}\end{array}\right.$ & $? 47,6 \mathrm{~m}$ & - & 1119 & Niedertrumersee ... & $\mathrm{N}$ & 500 & Inn-Donau & $\left\{\begin{array}{lll}4,1 & \mathrm{~km} & \mathrm{~L} \\
1,3 & \mathrm{Br}\end{array}\right.$ & $40 \mathrm{~m}$ & $19,1^{\circ} \mathrm{C}$ \\
\hline 85 & Lanhardsee. & $\mathrm{Z}$ & 530 & Drau & sehr klein & seicht & - & 110 & Nussensee .... & N & 598 & Traun-Donau & $\left\{\begin{array}{l}610 \mathrm{~m} \mathrm{~L} \\
210, \mathrm{Br} .\end{array}\right.$ & $15,5 \mathrm{~m}$ & $17^{\circ} \mathrm{C}$ \\
\hline 86 & Leopoldsteinersee . & $x$ & 619 & Enns-Donau & $\left\{\begin{array}{lll}1,4 & \mathrm{~km} & \mathrm{~L} . \\
0,5 & , \mathrm{Br} .\end{array}\right.$ & $32 \mathrm{~m}$ & $17^{\circ} \mathrm{C}$ & 111 & Obersee beim Königssee & N & 610 & Inn-Donau & $\begin{cases}1,3 \mathrm{~km} & \mathrm{~L} \\
0,45, & \mathrm{Br}\end{cases}$ & - & - \\
\hline 87 & Levicosee. & $s$ & 449 & Brenta & $\left\{\begin{array}{llll}2,48 & \mathrm{~km} & \mathrm{~L} \\
0,68 & & \mathrm{Br}\end{array}\right.$ & $35 \mathrm{~m}$ & - & 1112 & Vorderer Oberbergersee. & $N$ & - & ? Traun-Donau & - & - & - \\
\hline 88 & Lichtsee & 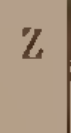 & 2100 & Inn-Donau & $\left\{\begin{array}{l}200 \mathrm{~m} \mathrm{~L} \\
150, \mathrm{Br}\end{array}\right.$ & wenige $\mathrm{m}$ & $9^{0} \mathrm{C}$ & 114 & $\left\{\begin{array}{l}\text { Odensee } \ldots \ldots \\
\text { Offensee } \ldots \ldots\end{array}\right.$ & $\begin{array}{l}\mathrm{N} \\
\mathrm{N}\end{array}$ & $\begin{array}{l}764 \\
651\end{array}$ & $\begin{array}{l}\text { Traun-Donau } \\
\text { Traun-Donau }\end{array}$ & $\begin{array}{r}300 \text { " Br. } \\
1000 \mathrm{~m} \mathrm{i} . \mathrm{D} \text {. }\end{array}$ & $\begin{array}{r}25 \mathrm{~m} \\
37,6 \mathrm{~m}\end{array}$ & - \\
\hline
\end{tabular}




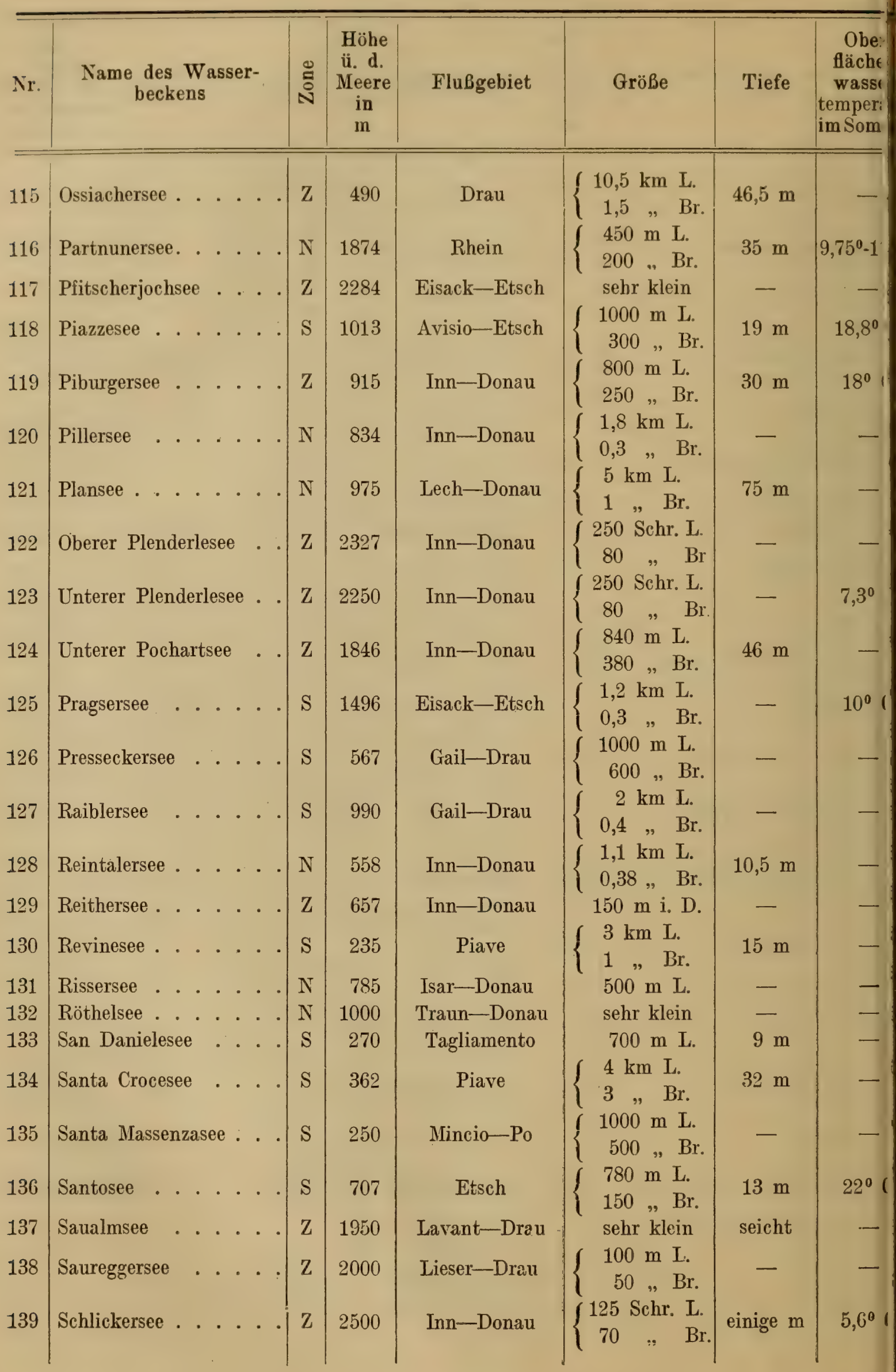




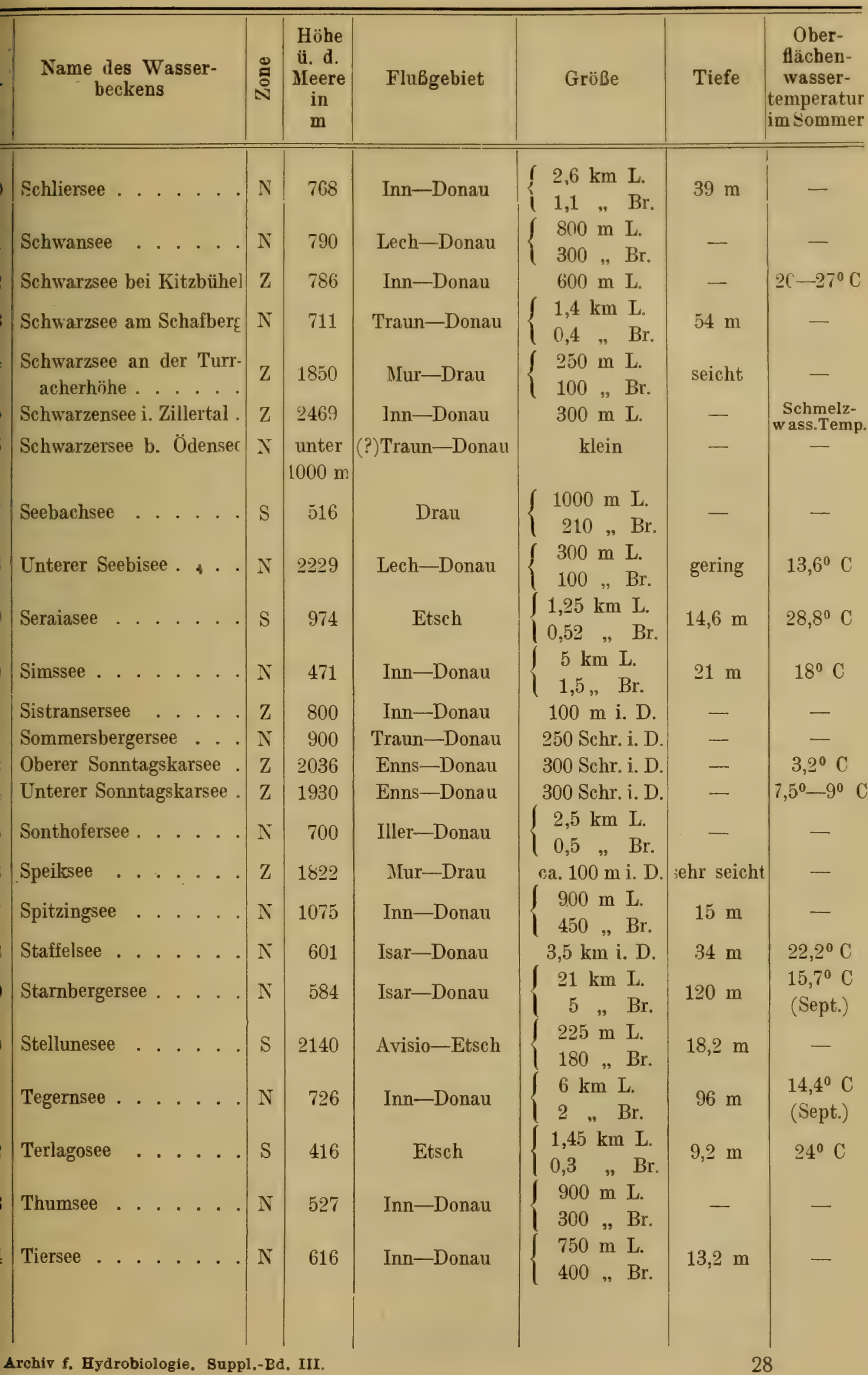





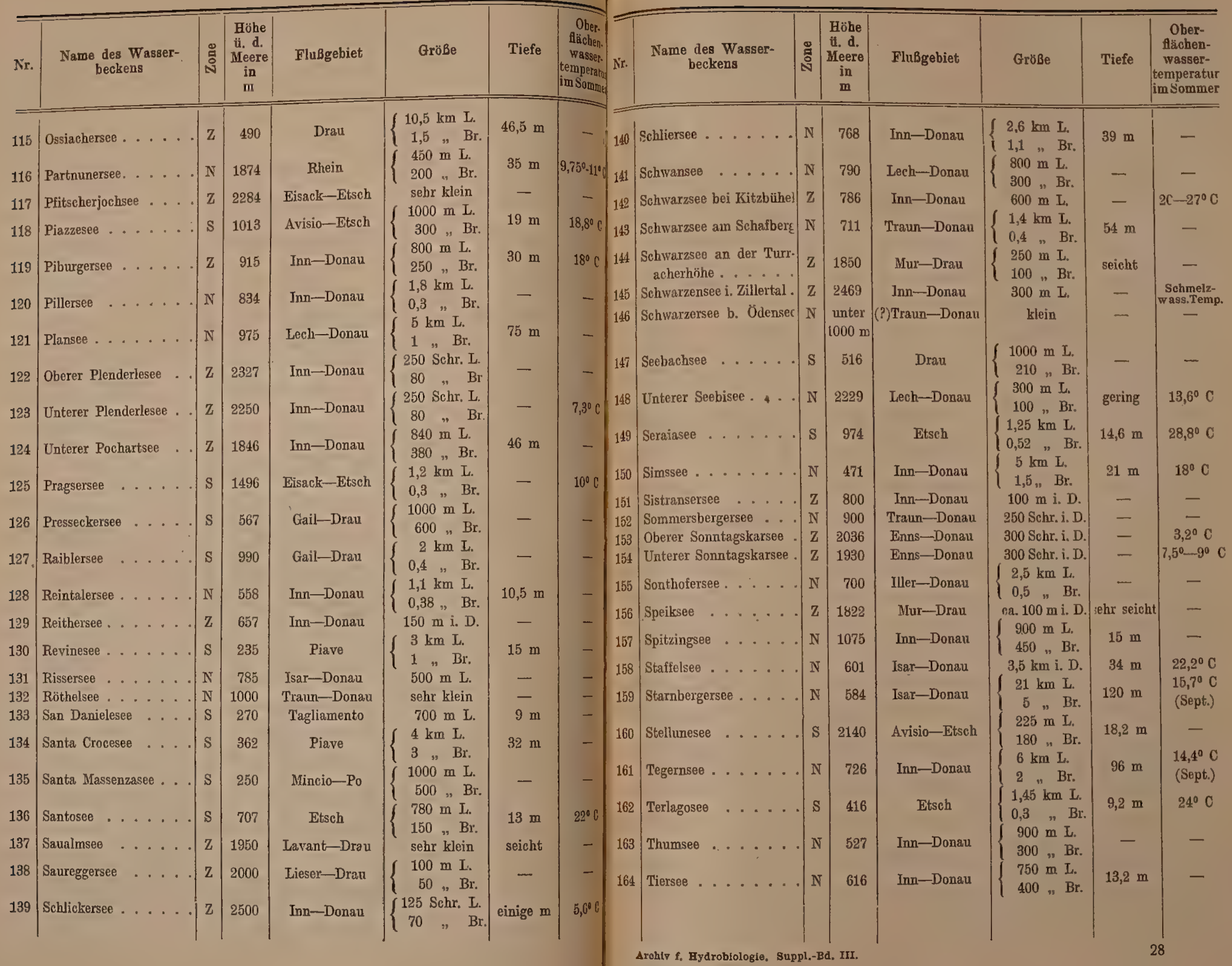




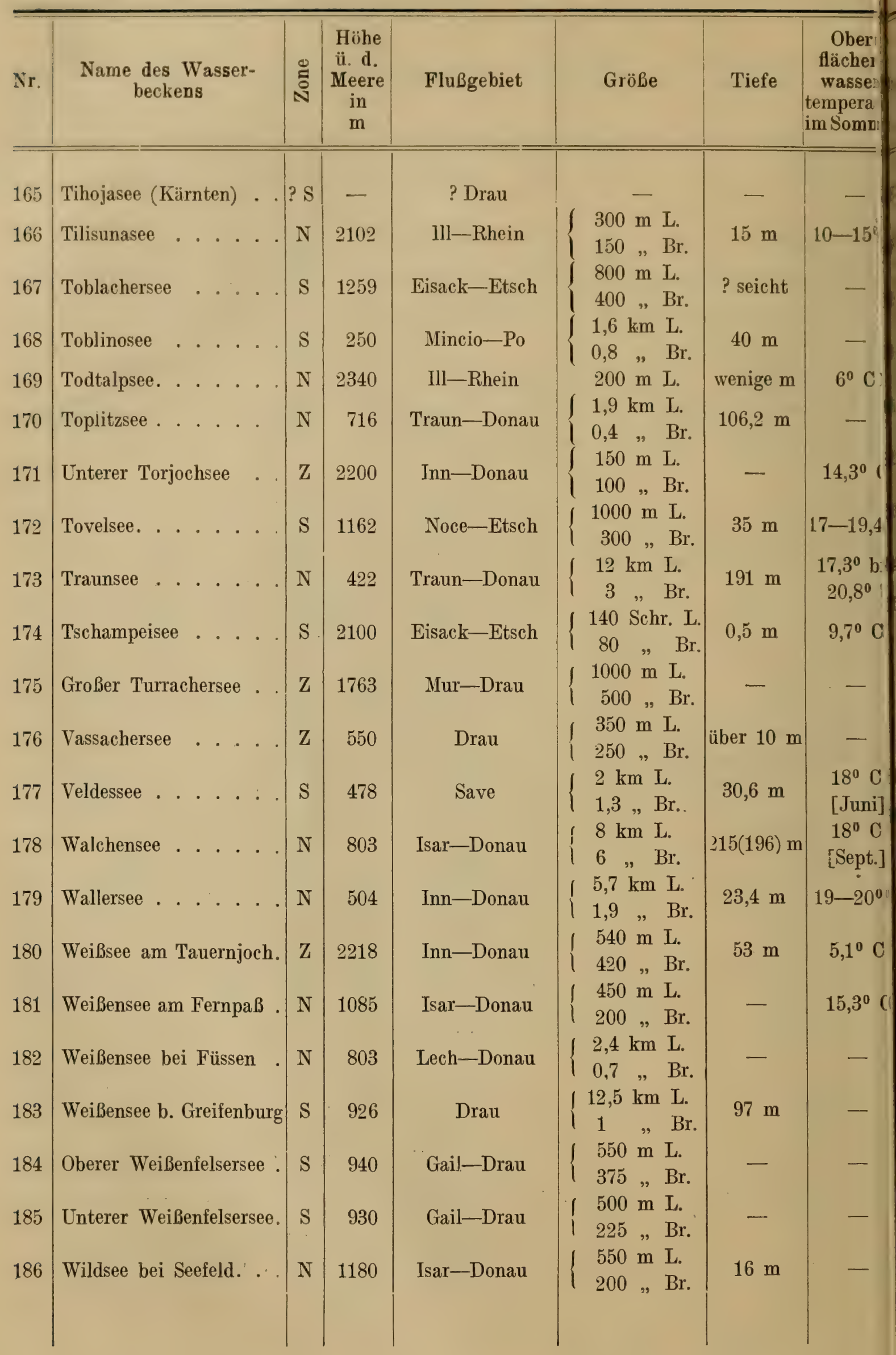




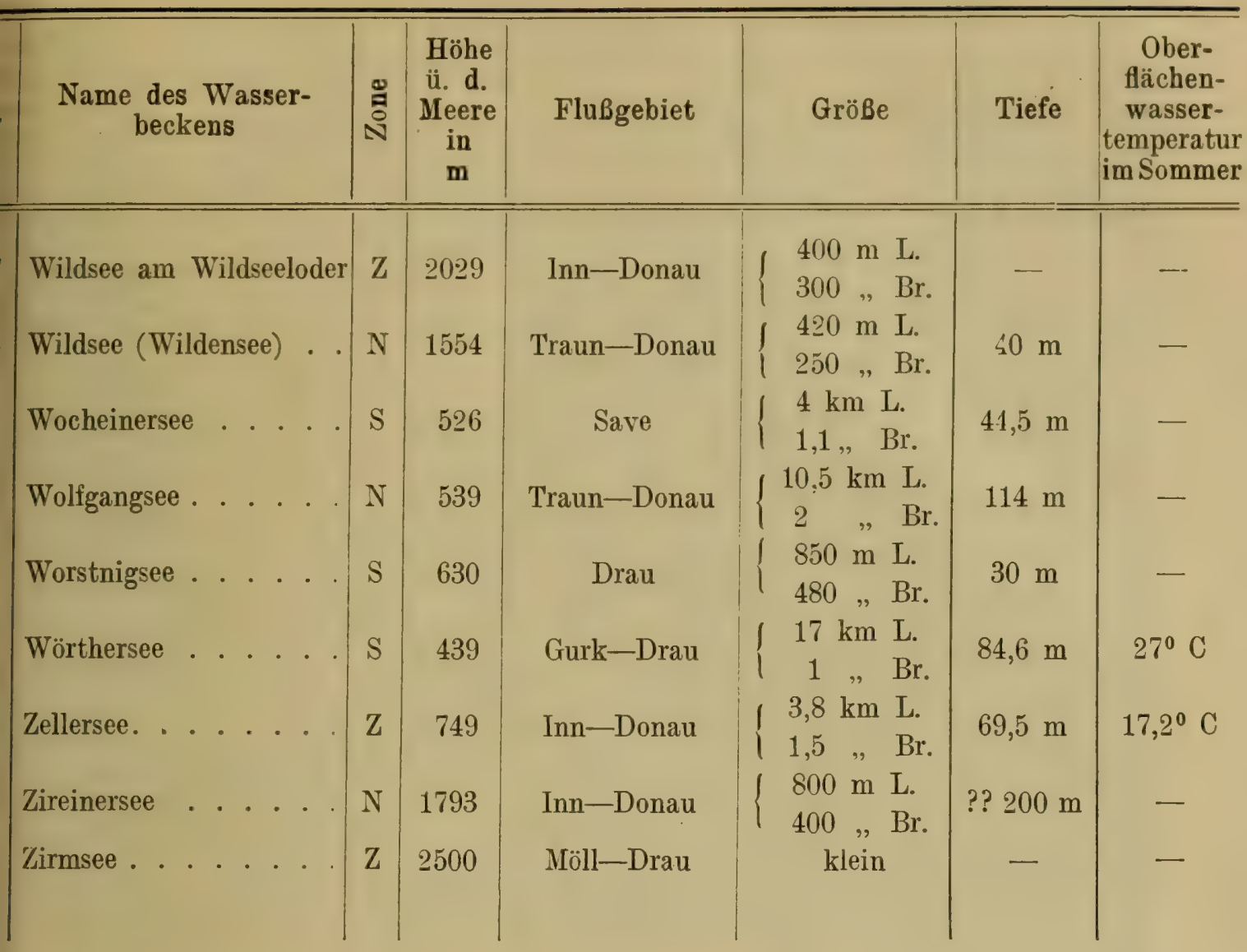

\section{Nachtrag.}

\begin{tabular}{|c|c|c|c|c|c|}
\hline Ammersee $\ldots$. & $\mathrm{N}$ & 533 & Isar-Donau & $\left\{\begin{array}{rll}16 & \mathrm{~km} & \mathrm{~L} \\
6 & \mathrm{Br} .\end{array}\right.$ & $79 \mathrm{~m}$ \\
\hline Mittersee am Schafberg & $\mathrm{N}$ & 1334 & Traun-Donau & $100 \mathrm{~m}$ i. D. & $7,6 \mathrm{~m}$ \\
\hline
\end{tabular}

IV. Abschnitt.

Beiträge zur hydrobiologischen Charakteristik der Ostalpenseen (197) unter Berücksichtigung ihrer Copepoden- und Cladocerenfauna (Spezieslisten).

Die folgende Zusammenstellung über $197^{1}$ ) auf ihre Copepoden- und Cladocerenfauna untersuchte Seen der Ostalpen weicht von der üblichen Form der Darstellung faunistischer Ergebnisse wesentlich ab. Dem

1) Davon entfallen 40 auf unsere eigene Bearbeitung; die Resultate über 27 Seen waren bisher noch nicht veröffentlicht. 



\begin{tabular}{|c|c|c|c|c|c|c|c|c|c|c|c|c|c|c|c|}
\hline Nr. & $\begin{array}{c}\text { Nime des Wasser- } \\
\text { berkens }\end{array}$ & 言 & $\begin{array}{l}\text { Höhe } \\
\text { i d } \\
\text { Mere } \\
\text { in } \\
\text { m }\end{array}$ & FluBgebiet & Grübe & Tiefe & $\begin{array}{l}\text { Obe. } \\
\text { Oläche: } \\
\text { wasce. } \\
\text { termpere. } \\
\text { im Sorne }\end{array}$ & & $\begin{array}{l}\text { Same des Wasser- } \\
\text { beckeus }\end{array}$ & 离 & $\begin{array}{l}\text { Hohe } \\
\text { it. d. } \\
\text { Meere } \\
\text { in } \\
\text { m }\end{array}$ & Flubgebiet & Grïbe & Tiefo & $\begin{array}{l}\text { Oher- } \\
\text { thichen- } \\
\text { wasser- } \\
\text { tenperatur } \\
\text { im Sommer }\end{array}$ \\
\hline 16:5 & Tihrojasere (Kärnten) & $\therefore$ & & & $300 \mathrm{~mL}$ L. & & & $1 \%$ & Willwe am Wildweluder & & 2011294 & $\operatorname{lnn-1)оnан~}$ & I t1114 in $\mathrm{I}$. & & \\
\hline $16 i j$ & Tilisunatep & $\mathrm{N}$ & 2102 & 1ll-Rhein & $\begin{cases}150 & \mathrm{Br} \\
800 \mathrm{~m} & \mathrm{~L} .\end{cases}$ & $\begin{array}{l}15 \mathrm{~m} \\
\text { ? seicht }\end{array}$ & & {$[\mathrm{s}$} & Wildere (Tildensee) & $x$ & 1.5.4t & Traun-Dhuau & & (1) $\mathrm{m}$ & \\
\hline 167 & Toblarhersee & $\mathrm{s}$ & 1259 & Eisack-Etsch & $\left\{\begin{array}{l}400 \ldots \mathrm{Br} \\
1,6 \mathrm{~km} \mathrm{~L}\end{array}\right.$ & $40 \mathrm{~m}$ & & 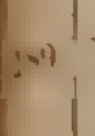 & Mocheinersee & s & 526 & Save & $\left\{\begin{array}{l}4 \mathrm{~km} \text { L. } \\
1,1 . . \mathrm{lir} .\end{array}\right.$ & $41,3 m$ & \\
\hline 1618 & $\begin{array}{l}\text { Toblinnere } \\
\text { Todtulpures. }\end{array}$ & $\begin{array}{l}5 \\
x\end{array}$ & $\begin{array}{r}250 \\
\pm 340\end{array}$ & $\begin{array}{l}\text { Mincio-Po } \\
\text { IIl-Rhein }\end{array}$ & $\left\{\begin{array}{l}0,8, B r \\
2(10 \mathrm{~m} \mathrm{~L} .\end{array}\right.$ & wenige $\mathrm{m}$ & 601 & $1 \cdot 4$ & Wolfgangere & $x$ & [3!!! & Traun- - Minlau & $\left\{\begin{array}{lll}l n .5 \mathrm{~km} & \mathrm{l} \\
\ddot{2} & \cdots & \text { lir. }\end{array}\right.$ & $114 m$ & \\
\hline 17 & Tupulitzene. & $\therefore$ & illi & Traun-Donau & $\left\{\begin{array}{l}1,9 \mathrm{~km} \mathrm{~L} \\
0,4, \mathrm{Br}\end{array}\right.$ & $106,2 \mathrm{~m}$ & & $a^{m !}$ & Worstnigsee & $s$ & 630 & Dran & $\left\{\begin{array}{l}850 \mathrm{~m} \mathrm{~L} \\
\mathrm{fin} \ldots \mathrm{Br}\end{array}\right.$ & $311 \mathrm{~m}$ & \\
\hline 17 & I'nterer Torjumbere & $\%$ & 20201 & Inn-Donau & $\begin{cases}150 \mathrm{~m} \mathrm{~L} \\
100 \ldots \mathrm{Br}\end{cases}$ & - & $14,3^{3}$, & 100 & Wörthersee & $s$ & 439 & Gurk-Drau & $\left\{\begin{array}{c}17 \mathrm{~km} \\
1 \ldots \\
1\end{array}\right.$ & $84,6 \mathrm{~m}$ & 9700 \\
\hline 172 & Tuvelsee. & $s$ & 162 & Suce-Etsch & $\left\{\begin{array}{r}1000 \mathrm{~m} \mathrm{~L} \\
300 \% \mathrm{Br} .\end{array}\right.$ & $35 \mathrm{~m}$ & & 1 & Zellersee. & Z & 749 & Inn- Donau & $\left\{\begin{array}{l}3,8 \mathrm{ku} \\
1,5\end{array}\right.$ & $6 ! 1, i) \mathrm{m}$ & 10,200 \\
\hline 173 & | Trambnte & $x$ & $4 \div 2$ & Traun-Donau & $\left\{\begin{array}{c}12 \mathrm{~km} \mathrm{~L} \\
3 \mathrm{Br}\end{array}\right.$ & $191 \mathrm{~m}$ & $\begin{array}{l}17,30 \mathrm{k} \\
20,80^{\circ}\end{array}$ & $19: 9$ & Zireinersee & N & 1793 & Inก Dกเล & 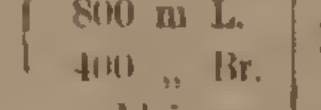 & & \\
\hline 174 & Tschampeisee . & $\mathrm{s}$ & 2100 & Eisack-Etsch & $\left\{\begin{array}{c}140 \text { Schr. L } \\
80 \% \mathrm{Br} \\
1000 \mathrm{~m} \mathrm{~L} \text {. }\end{array}\right.$ & $0,5 \mathrm{~m}$ & $9,7^{\circ} \mathrm{C}$ & 150 & Zarmsee . & 2 & 2600 & Môll-Drau & klein & - & - \\
\hline 175 & Großer Turrachersee . & $\mathrm{Z}$ & 1763 & Jur-Drau & $\left\{\begin{array}{r}1000 \\
500, \mathrm{Br}\end{array}\right.$ & - & & & & & & & & & \\
\hline 176 & Vassachersee .. & $\mathrm{z}$ & 550 & Drau & $\left\{\begin{array}{lll}350 & \mathrm{~m} & \mathrm{~L} \\
250 & \mathrm{Br}\end{array}\right.$ & über $10 \mathrm{~m}$ & 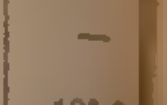 & & & & & Nachtrag. & & & \\
\hline 177 & bindersise & s & 48 & Save & $\left\{\begin{array}{l}2 \mathrm{~km} \mathrm{~L} . \\
1,3, \mathrm{Br}\end{array}\right.$ & & $\begin{array}{l}18^{\circ} \mathrm{C} \\
\text { [Jani] }\end{array}$ & 196 & Ammersee $\ldots \ldots$ & $\mathrm{N}$ & & Isar-Dnnau & $\mid \begin{array}{c}16 \mathrm{~km} \mathrm{k} \\
6, \mathrm{Br}\end{array}$ & $79 \mathrm{~m}$ & \\
\hline 178 & Walchensee... & $\mathrm{N}$ & 803 & Isar-Donau & $\mid \begin{array}{lll}8 & \mathrm{kmm} & \mathrm{L} \\
1 & 6 & \mathrm{Br}\end{array}$ & $315(196) \mathrm{m}$ & $\begin{array}{l}18^{\circ} \mathrm{C} \\
{[\text { Sept }]}\end{array}$ & & Suntersee am schalberg & $N$ & 1334 & Traun-Donau & $100 \mathrm{~m} \mathrm{i.} \mathrm{D.}$ & $7,6 \mathrm{~m}$ & - \\
\hline 179 & Wallersee & $\mathrm{N}$ & 504 & Inn-Donau & $\mid \begin{array}{lll}1,7 & \mathrm{~km} & \mathrm{t} \\
1,9 & \mathrm{Br}\end{array}$ & $23,4 \mathrm{~m}$ & $19-2,6$ & & & & & & & & \\
\hline 180 & Weißsee am Tauernjoch. & $\mathbf{Z}$ & 2218 & Inn-Donau & $\mid \begin{array}{lll}540 & \mathrm{~m} & \mathrm{~L} \\
420 & \mathrm{Br}\end{array}$ & $53 \mathrm{~m}$ & $5,1^{\circ} !$ & & & & & & & & \\
\hline 181 & WeiBensee am Fornpa B & $\mathrm{N}$ & 1085 & & $\left\{\begin{array}{l}450 \mathrm{~m} \mathrm{~L} \\
200, \mathrm{Br} .\end{array}\right.$ & - & $15,3^{\circ}$ & & & & IV. A & bschnitt. & & & \\
\hline 182 & WeiBensee bei Füissen & $\mathrm{N}$ & 803 & Lech-Donau & $\left|\begin{array}{lll}2,4 & \mathrm{~km} & \mathrm{~L} \\
0.7 & \mathrm{Br}\end{array}\right|$ & - & & & Beiträge zur hyd & Irob & biologi & ischen Char & kteristik dor & Ostsing & en. \\
\hline $1 \times 4$ & $\begin{array}{l}\text { WoiBensee b. Greifenburg } \\
\text { Oherer Weibinfolsersee }\end{array}$ & s & $\begin{array}{l}926 \\
940\end{array}$ & $\begin{array}{l}\text { Drau } \\
\text { Giail-Drau }\end{array}$ & $\left|\begin{array}{cccc}12,5 & \mathrm{~km} & \mathrm{~L} . \\
1 & \cdots & \mathrm{Br} . \\
550 & \mathrm{~m} & \mathrm{~L} \\
375 & \cdots & \mathrm{Br} .\end{array}\right|$ & $\begin{array}{r}97 \mathrm{~m} \\
-\end{array}$ & - & & $\begin{array}{r}\text { seen (197) unter } \\
\text { Cla }\end{array}$ & B & Beriick & $\begin{array}{l}\text { fachtigung } \\
\text { fauna (Spezi }\end{array}$ & $\begin{array}{l}\text { Wrer Copept } \\
\text { slisten). }\end{array}$ & oden- u & mil \\
\hline $1 \times 5$ & Unterer WeiBenfelserse. & $\mathbf{s}$ & 930 & Gail-Drau & $\left|\begin{array}{llll}500 & \mathrm{~m} & \mathrm{~L} \\
1 & 205 & & \mathrm{Br}\end{array}\right|$ & - & - & & $\begin{array}{l}\text { Die folgende Zu: } \\
\text { Cladocerenfauna unt }\end{array}$ & & $\begin{array}{l}\text { minensfe } \\
\text { uehte } \mathrm{S}\end{array}$ & $\begin{array}{l}\text { llung über } 197 \\
\text { ce'n der Ostally }\end{array}$ & $\begin{array}{l}\text { ") auf ihre cope } \\
\text { n weicht von e }\end{array}$ & $\begin{array}{l}\text { (cporden-" } \\
\text { der üblich }\end{array}$ & $\begin{array}{l}\text { hind } \\
\text { hind }\end{array}$ \\
\hline $1+i_{i}$ & Wildsee bei sirefeld. & $x$ & 1180 & Isar-Donau & $\mid \begin{array}{c}550 \mathrm{~m} \\
200 \\
200\end{array}$ & $16 \mathrm{~m}$ & - & & $\begin{array}{l}\text { Form der Darstellu } \\
\text { ') Davon entfalle } \\
27 \text { Seen waren bisher }\end{array}$ & en 1 & $\begin{array}{l}\text { faunis } \\
0 \text { anf o } \\
b \text { nicht }\end{array}$ & $\begin{array}{l}\text { tischer Lirg(e)s } \\
\text { tosere eigene Bet } \\
\text { veriffentlicht. }\end{array}$ & $\begin{array}{l}\text { josse wesentlich } \\
\text { rbeitong; die } R t\end{array}$ & all. D) & aber \\
\hline
\end{tabular}


diesen „Studien“ zugrunde liegenden Leitgedanken entsprechend, daß der Hydrobiologie im wahren Sinne vornehmlich die Aufgabe zufällt, die ursächlichen Beziehungen zwischen den Eigenschaften und Besonderheiten der Gewässer und dem Vorkommen und Auftreten ihrer Faunenelemente festzustellen, ist jeder Speziesliste eines Fundortes eine Reihe von Daten vorangestellt, welche über die geographische Lage, die Höhe über dem Meeresspiegel, das Flußgebiet, die Größe, die Tiefenverhältnisse, die geologisch-petrographische Beschaffenheit, die Topographie, die Hydrographie (Wassertemperatur), die allgemeine Entfaltung der Wasserflora und den Fischbestand des betreffenden Sees kurzen Aufschluß gibt. Mit der zugehörigen Copepoden- und Cladocerenliste sollen sich diese Angaben zu einer hydrobiologischen Charakteristik des Einzelfundortes vereinigen. Wenn dabei noch große Lücken zu konstatieren sein werden, so sind wir uns dieser Unvollständigkeiten voll bewußt; ihre Beseitigung konnte diesem ersten Versuch nicht gelingen. Daß die verschiedenen Autoren ihre faunistischen und häufig auch die hydrographischen Ergebnisse fast ausnahmslos durch Beobachtungen während der Sommermonate gewonnen haben, erhöht den Wert derselben für einen Vergleich der Fundorte untereinander bedeutend.

Alle in [] Klammern gesetzte Namen und Jahreszahlen beziehen sich auf ein entsprechendes Zitat im Literaturnachweis. Als nomenklatorische Grundlage für die in alphabetischer Anordnung abgefaßten Spezies!isten dienten die Bearbeitungen der Copepoden und Phyllopoden in dem Werke von Brauer „Die Süßwasserfauna Deutschlands“; wo dieselbe nicht ausreichte, wurden größere Spezialwerke (G. O. SARS, LillJeborg u. a.) benützt. Ein wiederholter Nachweis einer Spezies kann stets aus den beigefügten, in [] Klammern befindlichen Autorennamen (bezw. Jahreszahlen) entnommen und an der Hand des Literaturverzeichnisses nachgeprüft werden. In jenen Fällen, wo es sich um Synonyma handelte, ist der vom Autor gebrauchte Artname unter der Bezeichnung „sub" erwähnt; davon wurde nur bei allgemein bekannten und vollständig zweifelfreien Beispielen (so bei Leptodora, Sida) Abstand genommen.

Jene Zahl, welche als Fundortsnummer an erster Stelle jeder Charakteristik angeführt ist, findet sich in der beigegebenen Übersichtskarte der Ostalpen übereinstimmend eingetragen.

Abersee siehe Wolfgangsee (Nr. 190). 


\section{Achensee.}

F u n d ortsnu m mer: 1. Z o n e: Nördliche Kalkalpen. Nordtiroler Kalkalpen, an der Grenze der Karwendel- und Rissergruppe von der Brandenbergergruppe gelegen. G e o g r a ph i s c h e L a g e: Nordtirol, nördlich von Jenbach im Unterinntale. S e e h ö h e: $930 \mathrm{~m}$ ü. d. M. (? $944 \mathrm{~m}$ ). F l u B g e b i e t: Walchen-Isar-Donau. Gr ö B e: Großes, langgestrecktes Wasserbecken von $9 \mathrm{~km}$ Länge und $1 \mathrm{~km}$ Breite; $6,75 \mathrm{~km}^{2}$ Oberfläche (719 ha, $20 \mathrm{a}, 2 \mathrm{~m}^{2}$ ). N a x i maltief e: 138 (133) m; nach älteren irrtümlichen Angaben nur $92 \mathrm{~m}$ tief. Ge ologi s che und hydrographische Daten: Abdämmungssee durch Terrassenschotter (Stauschotter) aus dem Inntale. Nach Böнм [1886 p. 12] „entstand der Achensee infolge einer Absperrung des Achentales, die durch eine gewaltige Schotterablagerung im Inntale bewirkt wurde, mit welcher diejenige des Achenbaches, der damals in den Inn floB, nicht gleichen Schritt zu halten vermochte“. Liegt im Hauptdolomit; am Südufer Myophorienschichten [vergl. Dalla Torre 1913, p. 80]. Uffr steil und schroff, flache Ufer nur am Süd- und Nordende, sowie bei Pertisau. Seichtere Uferpartien in durchschnittlich $4 \mathrm{~m}$ Breite und bis zu 3 m Tiefe rund um den See, auffällig durch grüne Färbung, die Tiefenpartien des Sees azurblau. Uferboden meist steinig, Seegrund schlammig. Wassertemperatur: Mitte August an der Oberfläche $14,6^{\circ} \mathrm{C}$, in $60 \mathrm{~m}$ Tiefe $3,7^{\circ} \mathrm{C}$, in $120 \mathrm{~m}$ Tiefe $2,5^{\circ} \mathrm{C}$. Nakroskopische $\mathrm{W}$ a s s e r fior a sowie Phytoplankton vorhanden. Fischbestand: Perca fluviatilis, Cottus gobio, Scardinus erythrophthalmus, Lota vulgaris, Phoxinus laevis, Esox lucius, Thymallus vulgaris, Coregonus wartmanni, Salmo salvelinus, Trutta lacustris. - Die zooplanktonfreie, abyssale Region des Achensees liegt nach Hofer [1896] unterhalb $75 \mathrm{~m}$ Tiefe.

Die Copepoden- und Cladocerenfauna setzt sich nach den Untersuchungen von Imhof [1885], Hofer [1896], Brehm [1902], Zacharias [1903] und Brehu [1912, 1913] aus folgenden Spezies zusammen:

Co p e p od a: Canthocamptus cuspidatus Schmeil [Brenm 1912, 1913]. Cyclops albidus Jurine [Brehm 1912]. Cyclops strenuus Fischer [Hofer 1896, Brehi 1902, Zacharias 1903]. Diaptomus gracilis G. O. Sars [Hofer 1896]. (Nach Brehm [1902] kommt das Genus Diaptomus im Achensee nicht vor!)

Cl a d o c e r a: Acroperus sp. [Brehm 1912]. Alona affinis (Leydig) [Brehm 1912]. Alonella excisa (Fischer) [Brehm 1912]. Alonopsis elongata G. O. Sars [Brehm 1912]. Bosmina coregoni Baird (BurCK- 
hatdt) [Brehm 1902]. Bosmina coregoni var. lariana Burckh. [Zacharias 1903]. Chydorus piger G. O. Sars [Brehm 1912 ex Scourfield]. Chydorus sphaericus O. F. Müller [Brehm 1912]. Daphnia longispina var. hyalina LeYdig forma typica [BRehm 1902, ZACHARIAS 1903]. Eurycercus lamellatus (O. F. MülleR) [IмноF 1885, BRенм 1902, 1912]. Monospilus dispar G. O. Sars [ImhoF 1885 sub M. tenuirostris; Brehm 1912]. Peracantha truncata (O. F. MülleR) [BREHм 1902, 1912 sub Pleuroxus tr.]. Rhynchotalona falcata (G. O. SARS) [BREhm 1912]. Scapholeberis mucronata (O. F. Müller) [BReHu 1912]. Sida crystallina (O. F. Müller) [Brehm 1912]. Simocephalus vetulus (O. F. Müller) [Brehm 1912].

\section{Alleghesee (Lago d'Alleghe).}

F u n d o r t s n u m m e r: 2. Z o n e: Südliche Kalkalpen; Südtiroler Dolomiten, zwischen der Marmolata- und Civettagruppe. G e o gr a p h is e h e L a ge: Norditalien, Bezirk Belluno, südlich vom Caprilepaß. S e e h ö h e: 979 (976) mü. d.M. F l u B g e b i e t: Cocdevolebach-Piave. Größe: $2500 \mathrm{~m}$ lang, $550 \mathrm{~m}$ breit. Maximalt i e f e: $35 \mathrm{~m}$ (nach älteren Angaben ? $79 \mathrm{~m}$, ? $90 \mathrm{~m}) . \quad G$ e $0 \mathrm{log}$ is che und hydrogra p h is ch e D a t e n: Stausee durch Bergsturz [Böнm 1886, Halbrass 1913] am 11. I. und 1. V. des Jahres 1771 entstanden. B o d e n besehaffenheit: sandig. Wassertemperatur: kalt (? C-Grade); von November bis März gänzlich vereist. F i s c h b e s t a n d Cottus gobio, Salmo (Trutta) fario, Anguilla vulgaris.

Pavesi [1884] wies als Ergebnis seiner am 6. X. 1879 ausgeführten Planktonfänge folgende Spezies nach:

C o p e p o da: Cyclops serrulatus Fischer. Cyclops viridis Jurine [Pavesi sub C. gigas und C. brevicornis].

Cla d o r e ra: Daphnia longispina O. F. Nïüler. Daphnia pulex (DE GEer). Simocephalus vetulus (O. F. Mï̈Ller).

\section{Alpsee (Alpensee).}

F u n d or t s n u m mer: 3. Z o n e: Nördliche Kalkalpen; Ammerganergruppe der Nordtiroler Kalkalpen. Ge $0 \mathrm{gr}$ a $\mathrm{ph}$ is ch e L a g e: Südbayern, südöstlich von Füßen bei Hohenschwangau. S e e höh e: $815 \mathrm{~m}$ ü. d. M. Fl u Bg e b i e t: Seitental des Lech-Donau. Gr ö B e: größte Längenausdehnung ca. $1,7 \mathrm{~km}$, größte Breite ca. $800 \mathrm{~m}$. M a ximalti f f e: unbekannt.

Nach Імноғ [1887], dessen Fänge von den Monaten August und September stammen, enthält dieser See folgende Genera und Spezies: 
Co pepoda: Cyclops sp. Diaptomus sp.

Cla d o c e ra: Daphnia sp. Diaphanosoma brachyurum (LiÉvis). Leptodora Kindtii (Focke).

\section{Alpsee.}

Fundorts nu mmer: 4. Z o ne: Nördliche Kalkalpen; Bregenzer Waldgebiet der Allgäueralpen. G e $0 \mathrm{gr}$ a $\mathrm{ph}$ i s e h e L a g e: Südbayern, westlich von Immenstadt. S e e h ö h e: $664 \mathrm{~m}$ ü. d. M. Gr ö B e: mittelgroßes Wasserbecken von ca. $3 \mathrm{~km}$ Länge und $1 \mathrm{~km}$ größter Breite. Ma ximalti e fe: 25,5 m [nach Gerstbeck 1885]. Fl u B g e b i et: Achbach-Iller-Donau. G e o log i s ch e D a t e n: Durch Terrassenschotter abgedämmter See [Böнm 1886]. Die in den Monaten August und September ausgeführten Planktonfänge von Iмног [1887] ergaben den Nachweis folgender Copepoden und Cladoceren:

Co p e p o da: Cyclops sp. Diaptomus sp.

$\mathrm{C} l$ a d o c e r a: Bosmina sp. Daphnia longispina var. hyalina LEYDig. Diaphanosoma brachyurum (LIÉviv). Leptodora Kindtii (Focke).

\section{Altausseersee.}

Fundortsnummer: 5. Z o ne: Nördliche Kalkalpen; Salz kammergutgebiet. Ge $0 \mathrm{gr}$ a $\mathrm{ph}$ is ch e L a ge: Nordsteiermark, beim Bad Altaussee. S e e höh e: $709 \mathrm{~m}$ ü. d. M. Flu Bge bi et: Altausseer Traun-Traun-Donau. Gr ö Be: 2,09 km² Oberfläche; ca. $2,9 \mathrm{~km}$ lang, größte Breite $1,1 \mathrm{~km}$. Maxi malt i e f e: $52,8 \mathrm{~m}$; im Mittel 34,6 m tief. Ge ologische und hydrographiscbe Daten: Seeufer nach Halbfass [1913] teilweise von Endmoränen umgeben.

Die folgende Artenliste ist das Ergebnis der Untersuchungen ron Imhof [1885], Keissler [1902, 1907] und Langhans [1911].

Co p e p o da: Cyciops sp. [Imhof 1885, Keissler 1902]. Diaptomus sp. [Iмноғ 1885; Keissler 1902, 1907].

C l a d o c e r a: Bosmina sp. [Imhof 1885; KeIssler 1902, 1907]. Bosmina coregoni BAIRD (BurckHaRdT) $[=B$. longispina LeydiG, Langhans 1911]. Bythotrephes longimanus Leydig [Imhof 1885, Langhans 1911]. Daphnia sp. [KeIssler 1902, 1907]. Daphnia longispina O. F. Müller [Imhof 1885, Langhans 1911]. Daphnia longispina var. hyalina (Leydig) [Imнof 1885, Langhans 1911]. Leptodora Kindti (Focke) [ImhoF 1885, Langhans 1911]. 


\section{Anderlesee.}

(Bisher nicht veröffentlicht.)

Fu nd or t s n u m mer: 6. Z o ne: Zentralalpen; Norische Alpen. Geograph ische Lage: Nord-Kärnten, nahe der Grenze des Lungau (Salzburg); im Gebiet der „blutigen Alm" (Kremsbergeralpe) gelegen, Bezirk Gmünd i. K. S e e h ö h e: ca. 2000 m ü. d. M. Flu B g e bi e t: Weißenbach--Kremsbach-Lieser-Drau. Gr ö B e: rundlich geformtes Wasserbecken von ungefähr $250 \mathrm{~m}$ Durchmesser. M a x i m a lti efe: unbekannt. Geologisehe Beschaffenheit der $\mathrm{U} m \mathrm{~g}$ e b u $\mathrm{ng}$ : Zentralgneis. Wasserflora, insbesondere Fadenalgen, reich entwickelt.

Die Untersuchung der von Dr. K. Holdhaus im Monat August des Jahres 1920 ausgeführten Planktonnetzfänge ergab folgende Spezies:

C o p e p o d a: Cyclops serrulatus Fischer. Heterocope saliens LiLLJEBORG.

Cl a d o c e ra: Acroperus harpae BAIRD (forma aestivalis). Alona affinis (LeydiG). Chydorus sphaericus O. F. Mǘller. Daphnia pulex var. middendorffiana (FISCHER).

Kleiner See südlich vom Anderlesee.

(Bisher nicht veröffentlicht.)

Fundortsnummer: 7. Z o ne, ge o graphisehe Lage, Se ehöhe, Flu Bgebi et wi e oben (Nr.6). Größe: Kleiner Bergsee von ca. $100 \mathrm{~m}$ im Durchmesser. Ge ologie der Umg e b u n g: Zentralgneis.

Die im August 1920 von H. Dr. K. Holdhaus gesammelten Proben enthielten:

Copepoda: Cyclops serrulatus Fischer. Cyclops strenuus Fischer. Diaptomus sp. Heterocope saliens Lilljeborg.

Cladocera: Alona affinis (Leydig). Chydorus sphaericus O. F. Miüller. Daphnia longispina var. longispina (0. F. MǘlLer).

Kleiner See westlich vom Anderlesee.

(Bisher nicht veröffentlicht.)

Fundortsnummer: 8. Z o ne, ge ographisehe Lage, Se ehöhe, Flu Bgebiet wi e oben (Nr. 6). Größe: Kleiner Bergsee von ungefähr $100 \mathrm{~m}$ im Durchmesser. Ni a ximaltiefe: $1 / 2 \mathrm{~m}$ (schätzungsweise). 
Die von Herrn Dr. K. Holdhaus im August 1920 gesammelten Netzfänge enthielten:

Co p e poda: Diaptomus bacillifer Koelbel. Heterocope saliens LiLLJEBorg.

Cla d o cera: Alona affinis (Leydig). Chydorus sphaericus O. F. MüLler. Daphnia longispina var. longispina (O. F. Miüller).

\section{Attersee (= Kammersee).}

Fu ndortsnu mmer: 9. Z o ne: Nördliche Kalkalpen. Salzkammergutgebiet. Ge o $\mathrm{gr}$ a $\mathrm{ph}$ i s c h e L a g e: Oberösterreich, südlich von Vöcklabruck. S e e höh e: $465 \mathrm{~m}$ ü. d. M. F l u Bg e b i e t: AgerTraun-Donau. Grö Be: Größter österreichischer Alpensee im Ausmaße von $46,72 \mathrm{~km}^{2}$; ca. $19 \mathrm{~km}$ lang und von $2 \mathrm{~km}$ Durchschnittsbreite. Ma a i malti efe: $170,6 \mathrm{~m}$; mittlere Tiefe $84,2 \mathrm{~m}$. Ge ol o g is che und h ydrographische Daten: nach HalbFass [1913] stellt der Attersee ein inneralpines Zungenbecken dar, „das aber nirgends in das Kalkgebirge selbst hineinragt, sondern nur bis an seine steilen Abstürze hinausreicht". Makroskopische Wasserflora stellenweise reich entwickelt (höhere Wasserpflanzen und Algen). W a s s e r t e m p e r a t u r: an der Oberfläche Ende Juli und Anfang September bei $18^{\circ} \mathrm{C}$, in $10 \mathrm{~m}$ $14-17^{\circ} \mathrm{C}$, in $20 \mathrm{~m} 7^{0} \mathrm{C}$. B o d e $\mathrm{nbes} \mathrm{eh}$ a f f e n h e it: schlammig. $\mathrm{F}$ isch bestand: Alburnus bipunctatus, A. mento, Abramis brama, A. melanops, A. rimba, Barbus barbus, Coregonus fera, C. wartmanni, Cottus gobio, Cyprinus carpio, Esox lucius, Lotta lota, Lucioperca lucioperca, Nemachilus barbulata, Leuciscus meidnigeri, L. rutilus, Perca fluviatilis, Phoxinus phoxinus, Rhodeus amarus, Salvelinus salvelinus, Squalius cephalus, Scardinius erythrophthalmus, Trutta lacustris.

Über die Copepoder- und Cladocerenfauna des Attersees haben ImhoF [1885], Steuer [1901], Brunnthaler [1901], Keissler [1901], Brehy und Zederbauer [1906], Langhans [1911], Vitcoletzky [1912] und ВRенм [1913] berichtet; die Artenliste setzt sich folgendermaßen zusammen:

Co pepoda: Canthocamptus hoferi van Douwe [BRehm 1913]. Canthocamptus schmeili var. biserialis Mircoletzky [Micoletzky 1912, Brehu 1913]. Canthocamptus wierzejskii Mirázek [Mícoletzky 1912, Brenм 1913]. Cyclops sp. [Імноғ 1885]. Cyclops fimbriatus Fischer [Micoletzky 1912]. Cyclops fuscus Jurine [Mi. 1912]. Cyclops macrurus Sars [1i. 1912]. Cyclops oithonoides Sars [Keissler 1901, Brunnthaler 1901]. Cyclops serrulatus Fischer [Ni. 1912]. Cyclops strenuus 
Fischer [Kémsler 1901, Brehm und Zederbauer 1906, Brunnthaler 1901]. Cyclops viridis Jurine [M. 1912, Brunnthaler 1901]. Diaptomus sp. [Imнof 1885]. Diaptomus gracilis Sars [Brehm und Zederbauer 1906, Brunnthaler 1901]. Diaptomus graciloides Lillueb. [Steuer 1901, Keissler 1901, Brunnthaler 1901]. Diaptomus laciniatus Lilljeborg [BRUnNThaler 1901].

C l a d o c e r a: Acroperus angustatus Sars [Langhans 1911, Mircoletzky 1912]. Acroperus harpae Baird [Micoletzky 1912]. Alona affinis (Leydig) [Langhans 1911]. Alona costata Sars [Langhans 1911]. Alona guttata SARS [Langhans 1911]. Alona quadrangularis (O. F. Müller) [Langhans 1911, Micoletzky 1912]. Alona rectangula Sars [Langhans 1911, Micoletzky 1912]. Alonopsis elongata Sars [Langhans 1911, Micoletzky 1912]. Bosmina coregoni Baird [Steuer 1901]. Bosmina coregoni-longispina Leydig [KeIssler 1901 und BrunNthaler 1901 sub B. bohemica]. Bosmina longirostris (O. F. Müller) [STeuer 1901, Keissler 1901, Brunnthaler 1901]. Bosmina longirostrispelagica Stingelin [Keissler 1901 und Brunnthaler 1901 sub B. pelagica]. Bosmina sp. [Імноғ 1885̃]. Bythotrephes longimanus Leydig [Імноғ 1885, Keissler 1901, Langhans 1911]. Chydorus gibbus Lilljeborg [Micoletzky 1912]. Chydorus globosus Baird [Langhans 1911]. Chydorus latus Sars [Micoletzky 1912]. Chydorus sphaericus (O. F. Mï̈ller) [Keissler 1901, Micoletzky 1912]. Daphnia sp. [Імнof 1885]. Daphnia longispina var. hyalina Leydig [Brunnthaler 1901, Brehm und ZederbaUer 1906, Langhans 1911]. Diaphanosoma brachyurum (LiÉvin) [Імноғ 1885 sub Daphnella brachyura]. Diaphanosoma brachyurum var. leuchtenbergianum (FISCHER) [LANGHANs 1911]. Eurycercus lamellatus (O. F. Müller) [Mícoletzky 1912]: Graptoleberis testudinaria (Fischer) [Langhans 1911]. Leptodora kindti (Focke) [Imhof 1885, Keissier 1901, Langhans 1911]. Peracantha truncata (O. F. Müller) [MicoLetzky 1912]. Pleuroxus trigonellus (O. F. Mḯller) [Langhans 1911]. Sida crystallina (O. F. Müller) [Langhans 1911, Micoletzky 1912].

\section{Auerlingsee.}

(Bisher nicht veröffentlicht.)

F u n d orts n u m mer: 10. Z o n e: Zentralalpen; Norische Alpen. Ge ographische Lage: Steiermark, Bezirk Murau, südlich von St. Lambrecht im Gebiete der „Grebenzen“, nächst der Grenze gegen Kärnten gelegen. S e e h öh e: ca. 1500 m. F l u Bg e b i e t: Seitenbach der Mur-Mur-Drau. G ț ö Be: kleiner See von etwa 200 m Länge 
und 80-100 m Breite. G e o l o g i e der U m g e b u n g: Das Wasserbecken liegt im Quarzphyllit.

In den Planktonnetzfängen, die Herr Dr. K. Holdhaus am 30. VI. 1921 ausführte, wurden von uns folgende Spezies nachgewiesen:

C o p e p o d a: Cyclops serrulatus Fischer. Diaptomus gracilis Sars.

$\mathrm{Cl}$ a d o e e ra: Alona quadrangularis (O. F. lï̈ller). Chydorus sphaericus O. F. Müller. Diaphanosoma brachyurum (LiÉviv). Simocephalus sp.

\section{Augstsee.}

Fu n d o r ts n u m mer: 11. Z o n e: Nördliche Kalkalpen; Salzkammergutgebiet. Ge ograph is che Lage: Nordsteiermark, bei Altaussee am Fuße des Loser gelegen. S e e $\mathrm{h}$ ö e: $1633 \mathrm{~m}$ ü. d. M. Fl u B g e b i e t: Augstbach-Altausseertraun-Traun-Donau. Gr ö Be: Sehr kleines Becken von 0,009 $\mathrm{km}^{2}$ Oberfläche. Ti ef e: $30 \mathrm{~m}$ [vergl. MÜLLNer 1896].

Im Augstsee wurden von Langhavs [1911] folgende Cladoceren nachgewiesen:

Acroperus harpae BAIRD. Alona affinis (LEYDIG). Alona costata SARS. Alona guttata SARS. Alona quadrangularis (O. F. MüLler). Alona rectangula SarS. Daphnia longispina O. F. MüLler. Daphnia pulex (DE GEER) var. obtusa Kurz.

\section{Augstwiesensee.}

Fu nd ortsnum mer: 12. Z o n e: Nördliche Kalkalpen; Salzkammergutgebiet. Ge o gra ph is ch e L a ge: Nordsteiermark, nordöstlich von Aussee im Augstlogelgebiet (Totes Gebirge) gelegen. S e e h öh e: ca 1400 m ü. d. M. F l u Bg e bi et: Altausseertraun-TraunDonau. Grö Be: unbekannte Maße, sehr kleiner See.

Die Entomostrakenfauna des Augstwiesensees wurde von STEUER [1901] und Langhans [1911] untersucht und ergab folgendes Resultat:

C o p e p o d a: Heterocope saliens (Lilljeborg) [Steuer 1901].

Cl a d o e e ra: Alona affinis (Leydig) [Langhans 1911]. Alona rectangula SARS [LANGHANS 1911].

\section{Badersee.}

Fu n d o r ts nu mmer: 13. Z o ne: Nördliche Kalkalpen; Nordtiroler Kalkalpenzone; Wettersteingebiet. Ge $0 \mathrm{gr}$ a $\mathrm{ph}$ is c h e L a g e: Südbayern, westlich von Garmisch-Partenkirchen gelegen. S e e h ö h e: 
$830 \mathrm{~m}$ ï. d. M. Flu Bge bi et: Loisach-Isar-Donau. Grö B e: Kleiner See von ungefähr $200 \mathrm{~m}$ im größten Durchmesser. M a x i m a ltief e: $15 \mathrm{~m}$; Geisteeck [1885] gibt nur 5,2 $\mathrm{m}$ an; er maß am 12. VIII. 1881 eine Oberflächenwassertemperatur von $10,6^{\circ} \mathrm{C}$ und erwähnt, daß der See auch in den Wintermonaten eine Wassertemperatur von $6-7^{\circ} \mathrm{C}$ besitzt, daher nicht zufriere.

Nach dem Berichte Iмноғs [1887], der diesen See im August-September explorierte, enthielt derselbe keine pelagische Fauna. Später wurde seine Angabe durch BREHм [1907] bestätigt; auch dieser Autor konstatierte den Mangel eines Zooplanktons. Da jedoch im Badersee Fische existieren, so bedarf es für diesen Fundort dringend einer neuerlichen Untersuchung; nichtpelagische Entomostraken werden dem See wohl kaum fehlen.

\section{Bannwaldsee.}

F u n d o r t s n u m mer: 14. Z o n e: Nördliche Kalkalpen; Nordtiroler Kalkalpenzone; Ammergau. G e $0 \mathrm{graph}$ is c h e L a g e: Östlich von Füßen, Südbayern. Se ehöh e: $792 \mathrm{~m}$ ü. d. M. Fluß g e b i e t: Lech-Donau. Gr ö B e: Mittelgroßer Voralpensee von etwas mehr als $2 \mathrm{~km}$ Niaximallänge und $1 \mathrm{~km}$ Durchschnittsbreite. Mi a x i $\mathrm{m}$ a lt i e f e: $11 \mathrm{~m}$ (nach Angabe der Ostalpenübersichtskarte des D.Ö.A.V.).

Імног [1887] stellte dasVorkommen folgender Genera und Spezies fest:

Co p e p o da: Cyclops sp. Diaptomus sp.

Cl a d o c e r a: Bosmina sp. Daphnia sp. Diaphanosoma brachyurum (Liévin). Leptodora kindtii (FiocKE).

\section{Berghaustümpelsee.}

(Bisher nicht veröffentlicht ${ }^{1}$ ).

F u n d o r t s n u m m e r: 15. Z o n e: Zentralalpen; Norische Alpen. Ge 0 gr a ph i s e h e L a g e: Nordkärnten, am Fuße des Grünleitennock im Königsstuhlgebiet gelegen. S e e h öh e: ca. $1700 \mathrm{~m}$. Flu Bg e bi et: Kremsbach-Lieser-Drau. Gr ö Be: Tümpelartiges, jedoch perennierendes Wasserbecken von unbedeutendem Ausmaß. Ge $0 \mathrm{l} 0 \mathrm{~g}$ is c h e Beschaffenheit der Umgebung: Karbonisches Quarzkonglomerat.

1) Dieses sehr kleine Wasserbecken ist zwar auf den österreichischen Spezialkarten (1:75000) noch eingezeichnet, trägt jedoch keine Benennung; der obige Name ist von Herrn Dr. K. HoldHAus ad hoc, gelegentlich der Explorierung, geprägt worden und gründet sich auf das nahegelegene Knappenhaus (Berghaus). 
Die Untersuchung der von Herrn Dr. K. Holdhaus Ende August des Jahres 1921 ausgeführten Planktonnetzfänge ergab das Vorkommen folgender Spezies:

Co p e p o da: Cyclops fuscus Jurine. Cyclops vernalis Fischer.

$\mathrm{Cl}$ a d o e e r a: Alona affinis (Leydig). Alonella excisa (FISCHER). Chydorus sphaericus O. F. MiüLler. Daphnia longispina var. Tongispina (O. F. MülleR). Simocephalus vetulus (O. F. MüLleR).

\section{Berglsteinersee.}

Fundorts nu mmer: 16. Z o n e: Nördliche Kalkalpen; Nordtiroler Kalkalpenzone; Brandenbergergruppe. Ge o gra $\mathrm{ph}$ is che L a ge: Nordtirol, nordöstlich von Kramsach im Unterinntal. S e e höh e: ca. $740 \mathrm{~m}$ ü. d. M. Flu Bge biet: Brandenberger AcheInn-Donau. Grö Be: Kleiner Bergsee von etwa $300 \mathrm{~m}$ Länge und $200 \mathrm{~m}$ Breite. Maximaltiefe: $1 \mathrm{~m}$. Geologie der Umg e b u n g: Der See liegt im Gehänge der Voldöpperbergkalkwände, auf fluvioglazialem Urgesteins- und Kalkgerölle; entstand durch Stauung infolge eines Bergsturzes [MÜLLER 1905].

Brenм [1907] stellte folgende Spezies fest:

C o p e p o d a: Keine.

Cl a d o c e ra: Acroperus harpae BAIRD. Alona guttata SARS (sub Lynceus g.). Chydorus sphaericus O. F. M. Peracantha truncata (O. F. MÜLLER). Scapholeberis mucronata (O. F. MÜLLER).

Bockhartsee siehe Pochartsee (Nr. 124).

\section{Bodensee.}

Fundortsnummer: 17. Z o n €: Nördliche Kalkalpen; Allgäuer Alpen (Bregenzer Wald). Ge o gr a ph is ch e L a g e: Grenzsee zwischen Vorarlberg, Schweiz, Bayern. S e ehöh e: 398 m ü. d. M. Flu ß g e b i e t: Rhein. Gr ö B e: $69,2 \mathrm{~km}$ größte Länge und $13,7 \mathrm{~km}$ größte Breite; $538,52 \mathrm{~km}^{2}$ Oberfläche bei Mittelwasser. M a xi mal $\mathrm{t} \mathrm{j}$ ef e $\mathrm{n}$ : $308 \mathrm{~m}$, in der Verbindungslinie Friedrichshafen-ArbonRomanshorn gelegen. Ge $0 \mathrm{log}$ is che und h ydrographische Date n: Die Entstehung des Bodensees fällt nach Penck gleich derjenigen der glazialen Zungenbecken auf der Nordseite der Ostalpen in das Eiszeitalter; nichts verrät, daß er oder sein weites Becken vorher vorhanden gewesen sei [HALbFASS 1913]. In der Umgebung charakteristische Moränenlandschaften: W a s s e $f$ a r b e: Grundton grün (gelb 
und blau im Verhältnis von 27 : 73 Prozenten), im östlichen Teile durch Einwirkung der Rheinwässer stärker gelblich; Transparenz im Winter größer als im Sommer, sowie auch lokal verschieden. Was serte mperatur im Mittel: Wintermonate $3,9^{\circ} \mathrm{C}$, Frühjahr $6,7^{\circ} \mathrm{C}$, Sommermonate $17,8^{\circ} \mathrm{C}$, Herbst $11,9^{\circ} \mathrm{C}$ an der Oberfläche; Temperaturabnahme bei $16^{\circ} \mathrm{C}$ an der Oberfläche, $14^{\circ} \mathrm{C}$ in $5 \mathrm{~m}, 12^{\circ} \mathrm{C}$ in $10 \mathrm{~m}, 9^{\circ} \mathrm{C}$ in $15 \mathrm{~m}, 6^{\circ} \mathrm{C}$ in $20 \mathrm{~m}, 4^{\circ} \mathrm{C}$ in $25 \mathrm{~m}, 2,5^{\circ} \mathrm{C}$ in $30 \mathrm{~m}$, weniger als $2^{\circ} \mathrm{C}$ in $40-80 \mathrm{~m}$, weniger als $1^{\circ} \mathrm{Cab} 100 \mathrm{~m}$ Tiefe. $\mathrm{Ch}$ e $\mathrm{m}$ i s c h e Z u s a m m e nsetzung des Wassers: Gilt chemisch als ziemlich „rein“ und enthält als mineralogische Hauptbestandteile Karbonate und wasserhaltige Tonerdesilikate. Sauerstoffgehalt in den obersten Wasserschichten normal, von $5 \mathrm{~m}$ Tiefe an bedeutend abnehmend; ungebundene Kohlensäure an Menge sehr gering. Bodenbeschaffenheit größtenteils schlammig, kalzium-karbonathältig. F i s $\mathrm{h} \mathrm{b}$ e s $\mathrm{t}$ a $\mathrm{n} \mathrm{d}$ : Trutta lacustris, Tr. fario, Salmo salvelinus, Coregonus wartmanni, C. exiguus var. nüsslini, C. schinzii helveticus var. bodensis, C. acromus, Thymallus vulgaris, Perca fluviatilis, Esox lucius, Lota vulgaris, Silurus glanis, Anguilla vulgaris, Cyprinus carpio, Tinca vulgaris, Barbus fluviatilis, Chondrostoma nasus, Squalius cephalus, S. leuciscus, Abramis brama, Blicca björkna, Scardinius erythrophthalmus, Leuciscus rutilus, Alburnus lucidus, Cottus gobio, Cobitis barbulata, Gobio fluviatilis, Phoxinus laevis, Lucioperca sandra.

Die planktonische Entomostrakenfauna des Bodensees ${ }^{1}$ ) hat schon seit langem Bearbeitung erfahren; die Namen Leydig, P. E. MüLler, Weismann, Vosseler und Imhof seien hier nur genannt. Die folgende Liste, deren Vertreter teils eupelagische Spezies, teils litorale Planktonten darstellen, basiert auf den Untersuchungen von B. Hofer [1896], BurCKhaRDt [1900], Stingelin [1906] und van Douwe [1908]; die Arbeit des zweiten Autors lieferte auch eine sichere Grundlage zur Erkennung und Ausschaltung synonymer Spezies.

Co pepoda: Canthocamptus hoferi van Douwe [van Douwe 1908]. Canthocamptus minutus Claus [Hofer 1896]. Canthocamptus schmeili Mrazek [van Douwe 1908]. Cyclops fuscus Jurine [Hofer 1896 sub C. coronata]. Cyclops leuckarti Claus [H. 1896, Burckhardt 1900]. Cyclops strenuus Fischer [H. 1896, B. 1900]. Cyclops vernalis Fischer [B. 1900 ex Vosseler]. Cyclops viridis Jurine (var. caecus HofER) [H. 1896]. Diaptomus gracilis SARS [H. 1896, B. 1900]. Diaptomus

1) Eine Zusammenstellung über die Literatur und Arbeiten betreffend des Bodensees hat C. B. KLunzinger geliefert: „Ergebnisse der neueren Bodensee-Forschungen“ [Archiv f. Hydrobiol., vol. II (1907), p. 97]. 
laciniatus Lillu. [van Douwe 1908]. Heterocope weismanni IмноF [H. 1896 sub H. robusta, B. 1900].

Cla d o cera: Acroperus harpae Baird [Stingelin 1906, auch sub Alona affinis = Lynceus striatus LeYdiG]. Alona costata G. O. SARS [Stivgelin 1906]. Alona rectangula G. O. Sars [Strng. 1906]. Alonopsis elongata (G. O. SARS) [Stivg. 1906]. Bosmina coregoni var. longispina Leydig [Hofer 1896, Burckhardt 1900, Stingelin 1906]. Bosmina longirostris forma cornuta (JURINE) [STING. 1906]. Bythotrephes longimanus Leydig [H. 1896, B. 1900 ex Hofer]. Camptocercus lilljeborgi Schoedler [Stivg. 1906]. Ceriodaphnia quadrangula (O. F. Mï̈lLeR) [H. 1896]. Chydorus sphaericus O. F. Müller [Strivg. 1906]. Daphnia longispina O. F. MüLler [Sting. 1906]. Daphnia longispina var. hyalina LeydiG [H. 1896, B. 1900 sub D. hyalina; Sting. 1906]. Daphnia longispina var. hyalina forma eylmanni Burckh. [Strng. 1906]. Daphnia pulex (DE GEer) [H. 1896]. Diaphanosoma brachyurum (LiÉviv) [H. 1896 sub Daphnella br., B. 1900, ST. 1906]. Diaphanosoma brachyurum var. leuchtenbergianum forma frontosa LiLlJ. [ST. 1906]. Eurycercus lamellatus (O. F. MÜ̈LLER) [H. 1896 sub Lynceus l.]. Graptoleberis testudinaria (Fischer) [ST. 1906]. Latona setifera (O. F. MüLLER) [H. 1896]. Leptodora kindtii (FockE) [H. 1896. B. 1900 ex Hofer]. Macrothrix hirsuticornis NoRMan et Brady [Strvg. 1906]. Macrothrix laticornis (Jurine) [St. 1906]. Pleuroxus aduncus (JURINe) [ST. 1906]. Pleuroxus uncinatus BaIRd [ST. 1906]. Polyphemus pediculus (LinnÉ) [ST. 1906]. Rhynchotalona rostrata (КосH) [ST. 1906 sub Alona r.]. Scapholeberis mucronata (O. F. MÜLLER) [H. 1896]. Sida crystallina (O. F. Mï̈Ller) [H. 1896, ST. 1906]. Simocephalus vetulus (O. F. MüLleR) [H. 1896 sub $S$. sima].

\section{Großer Bödensee.}

F u n d o r ts n u m m er: 18. Z o n e: Südliche Kalkalpen; Gebiet der Südtiroler Dolomiten. Ge ographisehe Lage: Südtirol, Bezirk Sexten, im Dreizinnengebiet gelegen. S e e h ö h e: ca. $2300 \mathrm{~m}$ ü. d. M. Flu Bge b i e t: Bödenbach -Fischleintalbach-SextenbachDrau. Gr ö ße: Kleiner Bergsee von ungefähr 180 Schritten Maximallänge und 150 Schritten Breite Maximaltiefe: Wenige Meter. Geologische und hydrographische Daten: Das Seebecken liegt in Raiblerschichten [BlaAs 1902]. Ufer flach, als üppige, zum Teil von Wollgras (Eriophorum) bestandene Alpenweiden entwickelt, stellenweise versumpft. Makroskopische und mikroskopische Wasserflora reich entfaltet. Seeboden von schlammig-lehmiger Be- 
schaffenheit. Chemische Zusammensetzung des Seewassers siehe Kapitel II. Wassertemperatur: Dieselbe betrug am 6. VIII. 1911 um $10^{\mathbf{h}} 30^{\prime}$ a. m. an der Oberfläche $16,2^{\circ} \mathrm{C}$.

Das Wasserbecken enthält nach PEsta [1912] folgende Spezies:

C o p e p o d a: Cyclops serrulatus Fischer. Cyclops strenuus Fischer. Diaptomus gracilis SARS.

Cladocera: Alona affinis (Leydig). Chydorus sphaericus O. F. Müller. Daphnia longispina var. longispina s. str. (forma typica O. F. MüLlen). Polyphemus pediculus (Linné). Simocephalus vetulus (O. F. MÜLLER).

\section{Kleiner Bödensee.}

F u n d o r t s n u m mer: 19. Z o n €: Südliche Kalkalpen; Gebiet der Südtiroler Dolomiten. Ge ographische Lage: Südtirol, Bezirk Sexten, Dreizinnengebiet. S e ehöh €: ca. $2300 \mathrm{~m}$ ü. d. M. Fl u B g e b i e t: Bödenbach - Fischleintalbach — Sextenbach -Drau. G r ö ß e: Ungefähr 160 Schritte lang und 130 Schritte breit. MI a x i m a l$\mathrm{t}$ i e f e: Nicht gemessen, jedoch handelt es sich um einen ausgesprochenen Seichtsee. Geologische und hydrographische Daten: Wie oben. W a s s e r t e m p e r a t u r: Dieselbe betrug am 6. VIII. 1911 um $4^{\text {h }} 45^{\prime}$ p. m. an der Oberfläche $18,7^{\circ} \mathrm{C}$.

Es wurden von Pesta [1912] nachgewiesen:

C o p e p o da: Cyclops serrulatus Fischer.

Cladocera: Alona affinis (LEYDIG). Chydorus sphaericus (O. F. MüLler). Daphnia longispina var. longispina (O. F. Müuller). Polyphemus pediculus (Linné). Simocephalus vetulus (O. F. MÜLler).

\section{Boësee (Lago di Boë).}

Fundortsnummer: 20. Z o ne: Südliche Kalkalpen; Südtiroler Dolomiten, Sellagruppe. Ge ogra ph is che Lage: Südtirol, Bezirk Collfuschg, am Fuße der Südostabstürze der Boëspitze gelegen. S e e höh e: $2282 \mathrm{~m}$ ü. d. M. Flu Bge bi et: Sichtbarer Abfluß nicht beobachtet; das vermutlich in tieferen Lagen zutage tretende Wasser dürfte in das Campolungotal (Cordevolebach-Piave) abfließen. Größc: Größter Durchmesser etwa 130 Schritte, Umfang bei 400 Schritte. Maximaltiefe: Tiefer Trichtersee, genaue Maße nicht ermittelt. Geologisehe und hydrographisehe Date $n$ : Das Wasserbecken liegt in der Dolomitriffbildung des Ostabhanges der Sella mit angelagerten Raiblerschichten und Dachstein- 
kalken. Ufer mit Ausnahme der Südostseite senkrecht aufsteigende Felswände oder steil geneigte Geröllhalden. Seichtwasserzone von sehr geringer Breite. Bodenbeschaffenheit steinig (Geröll), wenig Sand. Wasser stark transparent (klar), intensiv blaugrün gefärbt. W a s s e r t e m p e r a t u r: An der Oberfläche in der Zeit von $1-2^{\mathbf{h}}$ p. m. (13. VII. 1913) gemessen $10,25-10,5^{\circ} \mathrm{C}$. Makroskopische Wasserflora als größerer Characeenbestand am Ostufer und als Algenüberzüge an Steinen entwickelt. Chemische Zusammensetzung des. Wassers siehe Absehnitt II.

Von Pesta [1914] wurden folgende Spezies nachgewiesen:

Cope poda: Cyclops serrulatus Frscher. Diaptomus bacillifer KOELBEL.

Cl a d o c e ra: Chydorus sphaericus O. F. Müller. Daphnia longispina var. longispina s. str. Daphnia pulex (DE GEER) var. obtusa KURZ.

\section{Brennsee.}

F u n d o r t s n u m m e r: 21. Z o n e: Zentralalpen; Norische Alpen, Gebiet der Afritzer Berge. Ge ographis che Lage: Kärnten, östlich des Millstättersees gelegen. S e e h ö h e: $742 \mathrm{~m}$. F l u B g e b i e t: Riegerbach-Lieser-Drau. Größe: Gestrecktes Wasserbecken von ca. 11/2 km Länge. Hydrographisehe Daten: Nach dem Berichte KeIsslen's [1904] „schwarz gefärbt“; die oberflächliche Wassertemperatur am 17. VIII. 1903 betrug ca. 20-24 C.

KeIssLer [1904] crwähnt nur das Vorkommen folgender Genera:

Co pe p oda: Cyclops sp. Diaptomus sp.

Cl a d o c e ra: Bosmina sp. Daphnia sp.

\section{Caldonazzosee.}

F u n d o r t s n u m mer: 22. Z o n e: Südliche Kalkalpen; Grenze zwischen dem Südtiroler Dolomitengebiet und der Vicentinischen Alpen des Etschbuchtgebirges. G e o gr a ph i s c h e L a g e: Südtirol, östlich von Trient im obersten Val Sugana gelegen. S e e h ö h e: 449 (441) m ü. d. M. F l u B g e b i e t: Brenta (Adria). G r ö B e: 4,2 km lang, $1,7 \mathrm{~km}$ breit; $5,38 \mathrm{~km}^{2}$ Oberfläche. Maxi malt i efe: Nach den Angaben von Brehm und Zederbauer [1904] ein Flachsee, dessen größte Tiefe mit $15 \mathrm{~m}$ bezeichnet wird; hingegen wird von älteren Autoren eine Maximaltiefe von $49 \mathrm{~m}$ genannt (vergl. dazu auch Dalla Torre [1913]. Geologische und hydrographische Daten: Stausee 
durch Schuttkegelbildung, einstmals zusammen mit dem Levicosee ein großes Wasserbecken bildend. Ufer meist flach, nur im Nordosten teilweise Steilhänge anstoßend; hohe Schilfbestände vorhanden. W a s s e rt e mperatur: An der Oberfläche während der Monate August und September 24,5-210 C gemessen, im Dezember $6^{\circ} \mathrm{C}$; friert nur unvollständig zu. Fischbestand: Zahlreiche Spezies (z. B. Aal, Barbe, Forelle, Hecht, Karpfen, Koppe, Neunauge, Pfrille, Rotfeder, Schleie, Stichling, Weißfisch u. a.).

Von Pavesi [1884], Largajolli [1898], Buffa [1902] und Brehm und Zederbauer [1904] wurde das Vorkommen folgender Copepodenund Cladocerenspezies nachgewiesen:

C o p e p o d a: Cyclops fuscus Jurine [Pavesi 1884 sub C. signatus, Largajolli 1898]. Cyclops serrulatus Fischer [Pavesi 1884, Largajolli 1898]. Cyclops strenuus Fischer [BRehm und ZederbaUer 1904]. Cyclops viridis JuRINe [Pavess 1884 sub C. brevicornis, LaRgajolli 1898].

$\mathrm{Cl}$ a d o c e r a: Alona quadrangularis (O. F. MḯlLER) [LARGaJolli 1898]. Bosmina sp. juvenis [Brehm und Zederbauer 1904]. Bosmina longirostris (O. F. Müller) [Pavesi 1884, Largajolli 1898, Buffa 1902]. Diaphanosoma brachyurum (LiÉvin) [von allen Beobachtern genannt]. Leptodora kindti (FockE) [von allen Beobachtern sub L. hyalina genannt].

\section{Cavazzosee (Lago di Cavazzo).}

F u nd ortsnummer: 23. Z o n e: Südliche Kalkalpen; Venetianeralpen. G e o gra ph is e h e Lage: Norditalien, Bezirk Gemona, im Südwestwinkel des Knies des Tagliamento gelegen. S e e h öh e: 195 m ü. d. Mi. Flu B g e b i e t: Tagliamento (Adria). Gr ö Be: $5 \mathrm{~km}$ lang, $1 \mathrm{~km}$ breit. Mi a x i m a l t i e f e: $41 \mathrm{~m}$. - Enthält an Fischen: Alburnus, Anguilla, Leuciscus, Petromyzon, Tinca.

Das Wasserbecken wurde von A. Senna [1890] am 21. V. 1889 nachts und am 23. V. 1889 tagsüber abgefischt; die Untersuchung der Proben ergab das Vorkommen folgender Spezies:

Cope poda: Cyclops sp. (juvenis). Cyclops diaphanus Fischer [Senna sub C. minutus Claus]. Diaptomus gracilis Sars var. carnicus Senna:

C l a d o e e r a: Bosmina coregoni Leydig [Senna sub B. longispina LEYDIG]. Bosmina longirostris (O. F. MüLLER). Ceriodaphnia quadrangula (O. F. MÜ̈LLER). 


\section{Ceisee (Lago di Cei).}

Fu n d orts n u m mer: 24. Z o n €: Südliche Kalkalpen; Sarcagruppe des Etschbuchtgebirges. Ge ographische Lage: Südtirol, Bezirk Rovereto, am Fuße der La Rochettaspitze. S e e höh e: 927 m ü. d. M. Fl u Bg e bi et: Val di Cei-Etsch. Gr ö ße: Nahezu 5 ha $\left(390,000 \mathrm{~m}^{2}\right)$ Oberfläche; $400 \mathrm{~m}$ lang, $150 \mathrm{~m}$ breit. M a x i m a l$\mathrm{t}$ i e f e: $6,55 \mathrm{~m}$.

Die Planktonnetzfänge, welche von Largajollr [1908/9] am 2. August des Jahres 1907 ausgeführt wurden, enthielten:

Co p e p o d a: Cyclops leuckarti Claus. Cyclops serrulatus Fischer. Cyclops strenuus Fischer.

$\mathrm{C} l$ a d 0 c e r a: Acroperus angustatus Sars. Alonella excisa (FISCHER). Alonella nana (BAIRD). Bosmina longirostris(-cornuta) JuRINE. Camptocercus rectirostris (Schoedler). Ceriodaphnia pulchella G. O. SARS. Diaphanosoma brachyurum (LiÉvin). Peracantha truncata (O. F. MiülLer). Sida crystallina (O. F. MǘLLER).

\section{Chiemsee.}

Fundort.snummer: 25.. Z o ne: Nördliche Kalkalpen; altbayerischer Alpenzug der Nordtiroler Kalkalpen. Ge o g r a $\mathrm{ph}$ i s c h e L a g e: Südbayern, östlich von Rosenheim. S e e h ö h e: $519 \mathrm{~m}$ ü. d. M. Flu ßg e bi et: Große Ache-Inn-Donau. Größe: Bedeutendster Voralpensee von $85 \mathrm{~km}^{2}$ Oberfläche; $14 \mathrm{~km}$ lang, $11 \mathrm{~km}$ breit. $\mathrm{M} \mathrm{a}$ ximalt i efe: $73 \mathrm{~m}$; mittlere Tiefe $24,47 \mathrm{~m}$. Ge o log i s e h e und h y drographise he Daten: Geistreck [1885] maß am 10. IX. 1881 eine Oberflächenwassertemperatur von $16,6^{\circ}$ C. Nach BREHм's Angabe betrug die $W$ assertemperatur der Oberfläche am 17. August $190418^{\circ}$ C. Hiezu vergleiche man die neueste Abhandlung von E. Scheffelt [1922].

Die folgende Liste der Planktonspezies gründet sich auf die Beobachtungen von Imhof [1888], Steuer [1901], Brehm [1906], Leuze [1913], Haempel [1913] i.nd Baumbach [1922]. Die Litoralfauna ist nicht untersucht worden.

Co p e poda: Cyclops leuckarti Claus [Brehm 1906, Haempel 1913, Baumbach 1922]. Cyclops strenuus Fischer [Leuze 1913, Haempel 1913, Baumbach 1922]. Diaptomus gracilis G. O. Sars [Brehm 1906, Leuze 1913, Baumbach 1922]. Heterocope weismanni Iмноғ [Імноғ 1888 sub H. saliens, Steuer 1901, Brehy 1906, Haempel 1913, BaumBACH 1922]. 
C I a d o c e r a: Bosmina coregoni Batrd [Brehm 1906, Baumbach 1922]. Bosmina longirostris (O. F. MüLler) [LEuze 1913]. Bythotrephes longimanus Leydig [Brehu 1906, Leuze 1913, Haempel 1913, BaumBACH 1922]. Daphnia longispina var. hyalina LEYDig [BREHM 1906, Leuze 1913, Baumbach 1922]. Diaphanosoma brachyurum (Liévin) [Brehm 1906, Leuze 1913, Baumbach 1922]. Leptodora kindti (Focke) [Brehm 1906, Haempel 1913, Baumbach 1922]. Peracantha truncata (O. F. Müller) [Baumbach 1922].

Außerdem führt LEuze [1913] die Gattung Lynceus sp. als vorkommend an. Zu Heterocope sei bemerkt, daß bereits Ввенм (op. cit. 1906, Richtigstellung p. 340) die Angaben über das Vorkommen der Spezies saliens im Chiemsee als irrtümlich bezeichnet hat und der in der Literatur da und dort aufgenommene Name durch weismanni zu ersetzen ist. SchefFElt [1922] fand außerdem noch folgende Spezies: Cyclops gigas Claus, Acroperus harpae Barrd, Alona sp., Ceriodaphnia sp., Chydorus sphaericus O. F. M., Sida crystallina (O. F. Mi.).

\section{Dürrensee.}

F u nd ortsnum mer: 26. Z o n e: Südliche Kalkalpen; Südtiroler Dolomiten. Ge o g r a ph is ch e L a e: Südtirol, Bezirk Toblach, westlich von den Dreizinnenspitzen. S e e h ö h e: 1410 (1406) m ü. d. M. Flu Bg e bi et: Rienz-Eisack-Etsch. Grö Be: 27,3 ha, $11 \mathrm{~m}^{2}$ Oberfläche; $500 \mathrm{~m}$ lang, $300 \mathrm{~m}$ breit. Ge $0 \mathrm{log}$ is ch e u nd h y drograph is che Daten: Ein Seichtwasserbecken (Stausee), das angeblich der jährlichen Austrocknung unterworfen ist (?). Ufer flach und stark verschilft. Brenm und Zederbauer [1905] maßen die oberflächliche Wassertemperatur am 3. VIII. 1903 mit $16^{\circ}$ C. Enthält Forellen.

Von den genannten Autoren werden lediglich folgende $\mathrm{C} l$ a d o c e r e $\mathrm{n}$ angeführt:

Daphnia longispina var. hyalina form. foreli (BuRCKHARDT). Lynceus sp. Scapholeberis mucronata (O. F. MïüLLE) f. fronte laevi (Lutz).

\section{Eibsee.}

F u n d o r t s n u m m e r: 27. Z o n e: Nördliche Kalkalpen; Wettersteingebiet der Nordtiroler Kalkalpen. Ge ographisch e Lag e: Südbayern, nächst der Grenze von Tirol, südwestlich von GarmischPartenkirchen gelegen. S e e höh e: $972 \mathrm{~m}$ ü. d. M. Flu Bg e bi et: Rohrbach-Loisach-Isar-Donau. Größe: 2,5 km lang, 0,8 km 
breit. Naximaltiefe: $28 \mathrm{~m}$. Geologisehe und hydrographische Daten: Nach BöHм [1886] ein echtes Felsbecken, welches durch Einsturz oder Auflösung des Kalkes entstanden ist.

Die Entomostrakenfauna des Eibsees wurde von Імноғ [1887] und zweimal von ВREHм [1907 und 1913] untersucht und lieferte bisher in sehr auffälliger Weise so spärliche Ergebnisse, daß es lohnend wäre, den Ursachen dieses liangels nachzuforsch€n; ВREнM konnte bei seinem ersten Explorierungsversuch überhaupt kein Crustaceenplankton nachweisen. Es sind bis heute nur zwei Spezies aus diesem See gemeldet, nämlich:

Canthocamptus minutus C'laus [Brehn 1913] und Leptodora kindti (FосKE) [IмноF 1887].

\section{Erlaufsee (Erlafsee).}

Fu n d o r t s n u m mer: 28. Z o n e: Nördliche Kalkalpen; österreichische Kalkalpen. Geographisehe $\mathrm{Lage:} \mathrm{Westlich} \mathrm{von}$ Mariazell, an der Grenze Niederösterreichs gegen Steiermark gelegen. S e ehöhe: 835 m ü. d. M. Flu Bge biet: Große Erlaf-Donau. Gr ö Be: $1200 \mathrm{~m}$ lang, $600 \mathrm{~m}$ breit. Ni a x i malt i e f e: $44 \mathrm{~m}$. Ge 0 logisehe und hydrographisehe Daten: Gehört zu den durch Endmoränen angestauten Seen. Seine Flachufer weisen Verschilfung auf. Nach BreHM und Zederbauer [1902] ist dieses Becken von Dezember bis April der Vereisung unterworfen. Die oberflächliche Wassertemperatur wurde am 7. VII. 1901 mit 16,50 C abgemessen.

Brehm und Zederbauer [1902] und Brehm [1913] konstatieren das Vorkommen folgender Arten:

Co p e poda: Canthocamptus wierzejskii lïrazeK [BRehm 1913]. Diaptomus gracilis G. O. Sars [Brehm und Zederbauer 1902]. Moraria schmeili VAN Douwe [BReHM 1913].

$\mathrm{Cl}$ a d o c e r a: Bosmina coregoni Baird [BREHM und Zederbauer 1902]. Bosmina longirostris (O. F. Müllier) [BRehm und Zederbauer 1902]. Chydorus sphaericus (O. F. Mǘletr) [Brehm und Zederbauer 1902]. Daphnia longispina var. hyalina LEYdig [BREHM und ZEDERbauer 1902]. Polyphemus pediculus (Linné) [Brehm und Zederbauer 1902]. Scapholeberis mucronata (O. F. MülLer) [BREHM und ZederBAUER 1902].

\section{Faakersee.}

Fun d o r t s n u m m e r: 29. Z o n e: Südliche Kalkalpen; Gebiet des Klagenfurter Beckens. Ge ogra ph is ch e L a g e: Westkärnten, 
südöstlich von Villach. S e e h ö h e: $560 \mathrm{~m}$ ü. d. M. Flu Bg e b i e t: Worounica-Gail-Drau. Größe: 2,345 $\mathrm{kn}^{2}{ }^{2}$ Oberfläche; $2,15 \mathrm{~km}$ lang, $1,7 \mathrm{~km}$ breit. M a x i m a l t i e f e: $29,5 \mathrm{~m}$; mittlere Ticfe: $14,25 \mathrm{~m}$. Geologisehe und hydrographische Daten: Das Secbecken stellt nach Richter [1897] eine zwischen großen Schuttkegeln ausgesparte Wanne dar, deren Ufer bereits an drei Seiten versumpft sind - die Vorstufe zur gänzlichen Verlandung; Inselbildung aus tertiärem Konglomerat bestehend.

Der See wurde untersucht von Imнor [1890], Steuer [1897] und Keissler [1906]; er enthält demnach folgende Spezies:

C o p e p o da: Cyclops sp. [Imhof 1890, Keissler 1906]. Cyclops leuckarti Claus [Steuer 1897]. Cyclops strenuus Fischer [STEuer 1897]. Diaptomus sp. [Iмноғ 1890, KeIssLer 1906]. Diaptomus gracilis G. O. Sars [Steuer 1897].

Cl a d o c e r a: Bosmina sp. [Imhof 1890, Keissler 1906]. Bosmina coregoni-longispina Leydig f. bohemica Sting. [STEUer 1897 sub Bosmina bohemica]. Chydorus sphaericus O. F. Mïüller [STEuer 1897]. Daphnia sp. [KeIssler 1906]. Daphnia longispina var. cucullata G. O. SARS f. kahlbergensis Schoedler [IмhoF $1890 \mathrm{sub}$ D. kahlbergensis, Steuer 1897]. Daphnia longispina var. hyalina LEydig f. galeata SARS [IмнoF 1890 sub D. galeata]. Diaphanosoma brachyurum (Lí́vin) [Iмно 1890 sub Daphnella, Steuer 1897]. Eurycercus lamellatus (O. F. MÜLler) [Steuer 1897]. Leptodora kindtii (FockE) [ІмноF 1890]. Scapholeberis mucronata (O. F. Müller) [Steuer 1897]. Sida crystallina (O. F. Mïüller) [Steuer 1897]. Simocephalus vetulus (O. F. MüLler) [STeuer 1897].

\section{Faistenauer Hintersee.}

F u nd ortsnum mer: 30. Z o n e: Nördliche Kalkalpen; Salzburger Kalkalpen (Salzkammergut). Ge o gra phische Lage: Salzburg, westlich von St. Gilgen am Wolfgangsee. S e e h ö h e: $685 \mathrm{~m}$ ü. d. M. Fl u B g e b i et: Almbach-Salzach-Inn-Donau. Gr ö B e: $1395 \mathrm{~m}$ lang, $720 \mathrm{~m}$ in maximaler Breite; $0,8229 \mathrm{~km}^{2}$ Oberfläche. $\mathrm{M} \mathrm{a}$ ximaltiefe: $22 \mathrm{~m}$. Ge ologisehe und hydrographische D a t e n: Nach FugGer [1891] ein gestauter Dammsee, nach HalbFass [1913] ein Grundwassersee, der keine regelmäßigen Zuflüsse besitzt und als der Rest jener Wassermengen angesehen werden kann, welche durch die Schotter des einst im Brunauertale sich erstreckenden Gletschers angestaut haben. Von Mitte Dezember bis Mitte März vereist; oberflächliche W a s s e r t e m p e r a t u r im Mittelwert während des Monates 
Juni $14^{\circ} \mathrm{C}$. Wasserfärbung grün, mit einem Stich ins Gelbe, "wie die Farbe der meisten im Kalkgebiet gelegenen subalpinen Seen" [Micoletzky 1910/11]. Geologische Beschaffenheit der Umgebung im Westen und Osten Hauptdolomit (Trias), im Norden älteres Quartär, im Süden Alluvium. Makroskopische Wasserflor a (Potamogeton, Chara) ebenso wie Schilfbestände (Carex, Scirpus, Phragmites, Equisetum) reich entwickelt, Wasseralgen und Phytoplankton zahlreich. F is ch b e st a n d: Cottus gobio L., Perca fluviatilis L., Phoxinus phoxinus (L.), Salvelinus salvelinus (L.), Trutta fario (L.).

Das Wasserbecken wurde sehr eingehend von Micoletzky [1910/11 und 1913] in den Monaten Juli-August 1909 und September 1910 untersucht; demnach beherbergt dieser See folgende Copepoden- und Cladocerenspezies:

C o p e p o d a: Canthocamptus crassus G. O. Sars [Micoletzky 1913]. Canthocamptus microstaphylinus WoLF. [M. 1910/11]. Canthocamptus staphylinus (JuRINe) [M. 1913]. Canthocamptus trispinosus BRADY [M. 1913]. Cyclops albidus Jurine [Mi. 1913]. Cyclops fuscus Jurine [M. 1910/11]. Cyclops macrurus G. O. SARS [M. 1913]. Cyclops serrulatus Fischer [M. 1910/11]. Cyclops strenuus Fischer [M. 1910/11]. Cyclops viridis Jurine [M. 1910/11]. Diaptomus gracilis G. O. SARS [M. 1910/11].

Cl a d o c e r a: Acroperus harpae BAIRD [M. 1910/11]. Alona affinis (LEYDiG) [M. 1910/11]. Alona quadrangularis (O. F. MüLLER) [M. 1913]. Alonella excisa (FIscher) [M. 1913]. Alonopsis elongata G. O. SARS [M. 1913]. Chydorus sphaericus O. F. MüLleR [M. 1910/11]. Daphnia longispina var. hyalina LEYDIG f. typica [M. 1910/11]. Graptoleberis testudinaria (Fischer) [M. 1910/11]. Peracantha truncata (O. F. Müller) [M. 1910/11]. Sida crystallina (O, F. MüLLER) [M. 1910/11]. Simocephalus vetulus (O. F. MüLLER) [M. 1910/11].

\section{Filzsee.}

(Bisher nicht veröffentlicht.)

F u n d o r t s n u m m e r: 31 . Z o n e: Zentralalpen; Niedere TauernGe o gr a ph is ch e Lage: Nordsteiermark, am Fuße des Höchstein. gipfels $(2544 \mathrm{~m}) \mathrm{im}$ Bereich der Schladminger Tauern südlich der Enns gelegen. S e e höh e: ca. $2100 \mathrm{~m}$ ü. d. M. Flu Bg e biet: Seewigbach-Enns-Donau. Grö Be: Kleiner Hochgebirgssee von zirka 100 Schritten in der Länge und 40 Schritten in der Breite. M a x i m a l ti efe: Schätzungsweise $5-6 \mathrm{~m}$. Ge ologisehe und hydro- 
gra ph is che 1 at a $n$ : Umgebung des Beckens durchwegs Urgestein, Kalke fehlen auch den im Niederschlagsgebiet liegenden Gipfeln. Das Seebett selbst stellt eine Geröllschuttmulde dar, dessen Umrandung im Süden und Westen steil aufsteigende, aus Fels- und Steintrümmern bestehende Hänge bildet, während die Nord- und Ostufer von flacheren Moosböden geringer Ausdehnung eingenommen werden. Seegrund mit zahlreichen Felsblöcken belegt, der schlammige Boden von der Beschaffenheit pines zerriebenen mineralogischen Detritus. Wasser klar und von dunkelgrüner Färbung. Makroskopisch sichtbare Was s e r flor a bis auf einen sehr gering entwickelten Algenbelag an Steinen fehlend. Wassertemperatur: Dieselbe betrug in der Zeit zwischen $9^{\text {h }} 45^{\prime}-11^{\text {h }}$ a. $m$. an der Oberfläche $7^{0} \mathrm{C}$ zum Besuchsdatum am 17. VIII. 1918. (Gleichzeitige Lufttemperatur $14^{0}$ C.) Als Wassertemperatur der drei kleinen Zuflüsse wurde zur selben Zeit festgestellt: $3^{\circ} \mathrm{C}, 3,2^{\circ}$ und $4,5^{\circ} \mathrm{C}$.

Die von uns vom Ufer aus ausgeführten Planktonnetzfänge enthielten nur folgende Spezies:

Cop e p oda: Cyclops sp. (iuvenis). Cyclops serrulatus Fischer.

Cla d o cera: Acroperus harpae BAIRD. Chydorus sp.

\section{Hinterer Finstertalersee.}

Fundortsnummer: 32. Z o n e: Zentralalpen; Rhätische Alpen; Gebiet der Ötztaler Alpen (im weiteren Sinne). Ge o graph is ch e L a g e: Nordtirol; südlich von Kühtai (Sellrain) bei Ötz im Ötztale. S e e h ö h e: 2259 m ü. d. Mi. Fl u B g e b i e t: Finstertalbach-Stuibenbach-Ötztalerache-Inn-Donau. Gr ö B e: ca. $300 \mathrm{~m}$ lang und $200 \mathrm{~m}$ breit. Maximalti e f e: Unbekannt. Ge ol o g is che und h y dr ographische Daten: Gneisgebiet. Das Seebecken nach Böнм [1886] und DALLA ToRRE [1913] ein typisches Gletschererosionsfelsbecken, zum größten Teile mit Schutt ausgefüllt. Oberflächliche Wassertemperatur am 21. VIII. $19019,5^{\circ} \mathrm{C}$ (bei gleich zeitiger Lufttemperatur von $12^{\circ} \mathrm{C}$ ). Enthält Forellen und Sa iblinge.

Brehm und Zederbauer [1904] melden das Vorkommen von zwei Planktonvertretern, nämlich: Cyclops sp. (iuvenis) und Chydorus sphaericus O. F. MÜLLER.

\section{Vorderer Finstertalersee.}

Fundortsnummer: 33. Im übrigen wie oben (Nr. 32). S e ehöh e: $2235 \mathrm{~m}$ ü. d. M. Größe: ca. $550 \mathrm{~m}$ lang, $300 \mathrm{~m}$ 
breit. Oberflächliche Wassertemperatur am 21. VIII. $1901 \mathrm{um} 9^{\mathrm{h}}$ a. m. $10^{\circ} \mathrm{C}$, in $5 \mathrm{~m}$ Tiefe $7^{0} \mathrm{C}$ (bei einer Lufttemperatur von $13^{\circ} \mathrm{C}$ ). E $\mathrm{nt}$ hält Forelle n und Sa ibling e. Brehm und Zederbauer ${ }^{\mathbf{1}}$ ) [1904] geben für ihre Planktonnetzfänge, die sich von $0-10 \mathrm{~m}$ Tiefe erstreckten, lediglich den Nachweis von Cyclops sp. (iuvenes, vermutlich zu strenuus Fischer gehörig) an.

\section{Foscagnosee (Lago di Foscagno).}

Fundortsnum mer: 34. Z o n e: Zentralalpen; Spölalpen der Rhätischen Alpenzone. Ge o g r a ph is c h e L a g e: Norditalien, Bezirk Bormio, Oberes Addatal (Valtellin) nächst der Schweizer Grenze. Seehöhe: $2235 \mathrm{~m}$ ü. d M. Flu Bgebiet: Viola-Adda-Po (Adria).

Copepoden- und Cladocerenfauna derzeit noch nicht veröffentlicht! (R. Miontr.)

\section{Fraẽlesee (Lago di Fraële).}

F u n d o r t sn u m mer: 35. Z o n e: Zentralalpen; Spölalpen der Rhätischen Alpenzone. Ge o gra ph is e h e Lage: Norditalien, Bezirk Bormio, oberstes Addatal nächst dem Fraëlepaß an der Schweizer Grenze. S e ehöhe: 1934 m ü. d. M. Flu Bge bi e t: Adda-Po (Adria).

Copepoden- und Cladocerenfauna derzeit noch nicht veröffentlicht! (R. Monti.)

\section{Fresenhalssee.}

F u n d o r t s n u m mer: 36. Zone: Zentralalpen; Norisehe Alpen; Gurktaleralpen. Ge o gra $\mathrm{ph}$ is e h e L a g e: Nordkärnten, am Fuße des Königsstuhls (Grenze zwischen Kärnten und Salzburg) gelegen. S e e h öh e: ca. $2150 \mathrm{~m}$ ü. d. M. Fl u Bg e b i et: Kamingbach-Riegerbach-Drau. Größe: Kleiner Hochgebirgssee (genauere Maße unbekannt). Maximal t i e f e: Auf 2-3 m geschätzt. Ge o lo g i s e h e und h ydrographis ch e Dat en: Liegt im Gebiete der kristal-. linen Schiefer. Seegrund meist sandig; Wasser klar, als Viehtränke benutzt.

1) Die auffällige Artenarmut an Entomostraken in den beiden bisher nur von BreHM und Zederbauer [1904] untersuchten Finstertalerseen, wo Forellen und. Saiblinge leben, dürfte vermutlich kaum dem wahren Sachverhalt entsprechen (vergl. dazu Plenderlesee sub Nr. 123); eine weitere Explorierung dieser Seebecken ist dringend: notwendig. 
Aus den von Dr. K. Holdhaus im Sommer des Jahres 1909 gesammelten Planktonnetzfängen konnten von uns [PESTa 1911] folgende Copcpodenspezies ermittelt werden:

C l a d o c e r a: Keine!

C o p e p o d a: Cyclops serrulatus Fischer. Cyclops strenuus Fischer. Heterocope saliens LILLJEBorg.

\section{Fuschelsee.}

Fu n d ortsnum mer: 37. Z o n e: Nördliche Kalkalpen; Salzburger Kalkalpen. Ge o g r a ph is c h e L a g e: Salzburg (Salzkammer-

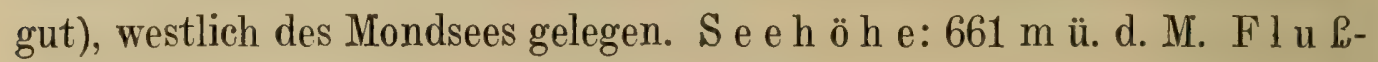
g e b i e t: Grieslerache-Ager-Traun-Donau. Gr ö B e: Langgestrecktes Becken von $4 \mathrm{~km}$ Länge und $800 \mathrm{~m}$ Breite; 2,66 $\mathrm{km}_{\mathrm{r}}^{2}$ Areal. $\mathrm{Mi} \mathrm{a}$ ximalt iefe: $67,3 \mathrm{~m}$; mittlere Tiefe $37,4 \mathrm{~m}$. Ge ologische und hydrographisehe Daten: Nach HalbFass [1913] als eine durch Moränenablagerungen angestaute Talstrecke anzusprechen.

Der See wurde am 25. VIII. 1884 von Iмноғ [1885] bezüglich seiner pelagischen Fauna untersucht und enthält demnach folgende Spezies:

C o p e p od a: Cyclops sp. Diaptomus sp.

Cl a d o e e ra: Bosmina sp. Daphnia longispina O. F. Müller. Diaphanosoma brachyurum (LIÉvin). Leptodora kindtii (Focke).

\section{Gafiensee.}

F u n d orts n u m mer: 38. Z o n e: Nördliche Kalkalpen; östlicher Rhätikon. Ge ograph i s che La ge: Schweiz, bei St. Anthönien im Gafientale. S e ehöh e: 2312 m ü. d. M. Flu Bgebiet: Dalvazzerbach-Landquart-Rhein. Gr ö Be: Sehr kleiner Hochgebirgssee mit einer Maximaltiefe von wenigen Metern. G e o lo g i s c h e und hydrographisehe Daten: Liegt im Grenzgebiet von Kalken und Schiefern. Vermutlich ein Quellsee; als oberflächliche Wassertemperatur maß ZscноккE am 8. VIII. 1892 bezw. 31. VIII. 1893 $7,5^{\circ} \mathrm{C}$ bezw. $10^{\circ} \mathrm{C}$.

Über die Entomostrakenfauna des Gafiensees haben SchmeIL [1893/95] und Zschoкke [1894] berichtet und folgende Spezies angeführt:

Co p e p oda: Canthocamptus rhaeticus SchmeIL. Cyclops strenuus Fischer. Cyclops vernalis Fischer.

Cla d o c e r a: Chydorus sphaericus O. F. MüLler. 


\section{Gaishornsee.}

Fu nd ortsnu m mer: 39. Z o ne: Zentralalpen; an der Grenze zwischen den Niederen Tauern und Eisenerzeralpen. G e $0 \mathrm{~g} r$ a $p$ h is $\mathrm{ch}$ e L a ge: Obersteiermark, Bezirk Rottenmann, Ortschaft Gaishorn. S e e höh e: ca. $700 \mathrm{~m}$ ü. d. M. Flu Bg e b i et: Paltenbach-EnnsDonau. Gr ö B e: $700 \mathrm{~m}$ lang, $250 \mathrm{~m}$ breit. G e $0 \mathrm{log}$ i s c h e D a t e r: Bӧнм [1886] zählt den See zu jenen Abdämmungsbecken, welche durch Schuttkegel von Wildbäehen und durch Muhrbruch entstanden sind.

Hartmann [1914] führt das Vorkommen folgender Cl a d o c e r a an, welche aus Planktonnetzfängen des Septembers 1913 stammen:

Acroperus harpae BAIRD (s. str.). Alona costata G. O. SARS. Ceriodaphnia megops G. O. SARS. Daphnia longispina O. F. MÜlleR (s. str.). Eurycercus lamellatus (O. F. MÜLLER). Lathonura rectirostris (O. F. Mï̈LLER). Peracantha truncata (O. F. MüLLER). Scapholeberis mucronata (O. F. MÜLLER). Scapholeberis mucronata forma cornuta (SCHOEdLER). Simocephalus vetulus (O. F. MÜLLER).

\section{Gardasee.}

F u n d or t s n u m m er: 40. Z o n e: Südliche Kalkalpen; Etsclbuchtgebirge. Ge ograph is ch e Lage: Südtirol-Italien. S e e höhe: $65 \mathrm{~m}$ ü. d. M. Flu Bgebiet: Sarca-Mincio-Po (Adria). Gr ö B e: $55 \mathrm{~km}$ lang, größte Breite $18 \mathrm{~km} ; 370 \mathrm{~km}^{2}$ Oberfläche. M a ximaltiefe: $346 \mathrm{~m}$. Ge ologis che und h ydrographische $\mathrm{D}$ a $\mathrm{t}$ e $\mathrm{n}$ : Nach BLAAs [1902] steht der Gardasee in einer unverkennbaren Beziehung zum Gletscherphänomen, wie die Gletscherschliffe an den Berghängen, die Reste von Seitenmoränen (besonders am Monte-BaldoAbhang) und das Moränenamphitheater, das den See im Süden umgibt, beweisen. Die Moränen erscheinen hier in fünf, den See in konzentrischen Ringen umgebenden Wällen. Die Moränen und Schotter bestehen vorherrschend aus Kalkgeröllen, aber auch nicht selten aus kristallinen Gesteinen (Granit, Gneis, Glimmerschiefer, Porphyr). Die Hauptmasse dieser Moränenbildungen fällt in die Zeit der letzten Vergletscherung, während die Anhäufungen älterer Glazialzeiten größtenteils unter jenen begraben liegen. HalbFass [1913] hält den Gardasee für ein zum großen Teile durch Moränen und Schotter abgedämmtes Becken, dessen Wanne selbst jedoch für eine vom alten Etschgletscher erodierte Furche. W a s s e r fa r b e: Blau; nach Dalla Torre [1913] durch die Reinheit des Wassers zu erklären (im Gegensatz zur grünen Färbung der Seen des Salzkammergutes). Ufer am Nordende steil und hoch, nach Süden 
zu sich allmählich verflachend. W a ssertemperatur: An der Oberfläche $19^{\circ} \mathrm{C}$, in $20-30 \mathrm{~m}$ Ticfe $13^{\circ} \mathrm{C}$, am Grunde $7,7^{\circ} \mathrm{C}$. Keissler [1906] maß a m 5. VIII. 1906 bei Riva eine oberflächliche Wassertemperatur von $22^{\circ} \mathrm{C}$. W a s s e f l o r a: Zahlreiche Algen (389 Spezies) und verhältnismäßig wenig Phytoplanktonformen, davon viele tycholimnetisch. Enthält zahlreiche F i s c h e: Neunauge, Aal, Seeforelle, Hecht, Schmerle, Weißfisch, Schleie, Pfrille, Rotfeder, Barbe, Karpfen, Stichling, Grundel, Koppe u. a.

Die folgende Artenliste der Copepoden und Cladoceren des Gardasees gründet sich auf die Arbeiten von Pavesi [1884], Garbini [1895], Brehm und Zederbauer [1904], Zacharias [1905] 1), Brehm [1906] und KeIssLer [1906].

Copepoda: Canthocamptus minutus Claus [Garbini 1895]. Cyclops fuscus Jurine [Garbini 1895 sub C. signatus] ${ }^{2}$ ). Cyclops leuckarti Claus [Brehm und Zederbauer 1904, Zacharias 1905]. Cyclops strenuus Fischer [Garbini 1895 sub $C$. quadricornis, Brehm und Zederbauer 1904, Brehm 1906]. Diaptomus sp. [Keisstar 1906]. Diaptomus castor (Jurine) [Pavesi 1884, Garbini 1895]²). Diaptomus steueri Brehm et Zederbauer [Garbini 1895 sub D. gracilis; Brehm und Zederbauer 1904; Zacharias 1905 sub D. graciloides; BREHM 1906]²).

C l a d o c e r a: Alona quadrangularis (O. F. MïulLer) [Garbini 1895 sub Lynceus quadrangularis]. Bosmina coregoni BAIRD var. amethystina Brehm [GARBINI 1895 sub B. longicornis; BREHM 1906]. Bythotrephes longimanus Leydig [GARBINi 1895]. Chydorus sphaericus O. F. Mïüller [GARBINI 1895]. Daphnia longispina var. hyalina LEYDIG f. pavesii Burckhardt [Pavesi 1884 und Garbini 1895 sub D. galeata et D. kahl-

1) ZACHARIAS op. cit. 1905 hat seine Angaben über das Vorkommen der Planktoncladoceren im Gardasee aus BREHM und ZEDERBAUER [1904] entnommen und nur die Copepoden C. leuckarti und Diatomus steueri, den er für graciloides hielt, selbst beobachtet.

2) Zunächst sei darauf hingewiesen, daß die von Pavesi und Garbini aus dem Gardasee gemeldete Spezies Diaptomus castor (JURINE) - ebenso wie Cyclops fuscus JURINE - in den Arbeiten der späteren Untersucher überhaupt nicht mehr erwähnt wird; es liegt jedoch bezüglich dieser beiden Copepoden kein Grund vor, an eine Verwechslung mit anderen Spezies zu denken und,ihr Vorkommen im Gardasee zu bezweifeln. Hingegen muß die Angabe des Vorkommens von Diaptomus gracilis G. 0. SARs nach den Ausführungen von BreHM und Zederbauer [1904] als irrtümlich bezeichnet werden; demnach kann es sich bei dieser Spezies auch nicht um den von BURCKHARDT vermuteten $D$. graciloides var. padana handeln, sondern um eine neue, dem $D$. gracilis nahestehende Art („Lokalrasse"!), welche von den genannten Autoren als $D$. steueri diagnostiziert wurde. 
bergensis; Brehm und Zederbauer 1904 und Brehm 1906 sub D. pavesii Burckhardt]. Daphnia pulex (de Geer) [Garbini 1895]. Diaphanosoma brachyurum (LiÉvin) [Pavesi 1884, Garbini 1895, Brehm und Zederbauer 1904]. Leptodora kindtii (Focke) [Pavesi 1884, Garbini 1895, Brehm und Zederbauer 1904]. Polyphemus pediculus (Linné) [Garbini 1895̃]. Scapholeberis mucronata (O. F. Müller) [Brehm und ZederbaUer 1904]. Sida crystallina (O. F. Müller) [Garbini 1895̃].

\section{Garschinasee.}

Fu n d orts n u m mer: 41. Z o ne: Nördliche Kalkalpen; Rhätikongebiet der Allgäueralpen. G e $0 \mathrm{~g} r$ a $p$ h i s e h e L a g e: Ostschweiz, im Bereich der Sulzfluh nördlich von St. Anthönien gelegen. S e e h öh e: 2189 m ü. d. M. Flu Bg e b i e t: Dalvazzerbach-LandquartRhein. Gr ö Be: $200 \mathrm{~m}$ lang, $100 \mathrm{~m}$ breit. M a x i malt i e fe: $3 \mathrm{~m}$. Geologische und hydrographische Daten: Der See liegt nach Zschокке [1890] im Schiefergebiet; als Oberflächenwassertemperatur wurde vom selben Autor im August des Jahres 1889 und $189014,5-16^{0} \mathrm{C}$ gemessen.

Das Becken enthält nach den Berichten von Zsснокке [1890, 1891 und 1900] und den von Schmeil [1893] publizierten Korrekturen und Ergänzungen folgende Spezies:

Co p e p o da: Canthocamptus zschokkei Schmeil [ZschoккE 1900]. Cyclops serrulatus Fischer [Zschokke 1890, Schmeil 1893]. Cyclops strenuus Fischer [ZschoKKe 1891] (iuvenis). Cyclops vernalis Fischer [Schmeil 1893]. Diaptomus denticornis Wierz. [ZschoKKe 1890 sub D. castor; Zschокке 1891 sub D. juvenis; SchmeIL 1893].

Cla d o c e r a: Alona quadrangularis (0. F. Müller) [ZschoккE 1890]. Daphnia longispina O. F. MüLler [ZschoкKe 1891] (iuvenis). Rhynchotalona rostrata (Косн) [ZschoKke 1890 sub Alona rostrata].

\section{Oberer Giglachsee.}

\section{(Bisher nicht veröffentlicht.)}

F u n d o i t s n u m m e r: 42. Z o n e: Zentralalpen; Niedere Tauern. Ge ogra phis ch e Lag e: Obersteiermark, südlich von Schladming am Fuße des Hading. S e e h ö h e: ca. $1960 \mathrm{~m}$ ü. d. M. Fl u B g e b i e t: Giglerbach-Talbach-Enns-Donau. Gr ö B e: Ungefähr $390 \mathrm{~m}$ lang und $150 \mathrm{~m}$ breit. Maximalti e fe: Unbekannt, Ufertiefen $2-5 \mathrm{~m}$. Geologische und hydrographische Daten: Der obere 
Giglachsee liegt in einer offenen, während der Sommermonate Juli und August durchschnittlich fast 12 Stunden voller Besonnung ausgesetzten, flachen Mulde. Mit Ausnahme der Südostseite, wo Geröllhalden vom Hadingkamm bis zum See reichen, erscheint er von flachen Ufern umrandet, bestehend aus begrastem Almboden mit verstreut umherliegenden Felsblöcken. Es wurden fünf Zuflüsse konstatiert. Die Geologie der Umgebung ist insoferne sehr interessant, als sich am Anstieg gegen den sogenannten Znachsattel eine ungewöhnlich scharf hervortretende Scheidung zwisehen Urgestein (Stock der Vetternspitzen mit Hading als Vorberg) und dem Kalkgebirge (Zug der Lungauer- und steirischen Kalkspitzen) ausprägt, die dem Beobachter schon weithin durch die verschiedene Vegetation bemerkbar wird. - Die im allgemeinen gering geneigten Uferränder zeigen steinigen Seegrund; die Bodenflächen werden meist von stark zerkleinertem mineralogischem Detritus eingenommen, nur selten finden sich Stellen von schlammartiger Beschaffenheit. Besonders günstige Stellen für den Planktonfang vom Ufer aus bietet der Westrand des Wasserbeckens, wo in geringer Entfernung vom Uferrand steil abfallende Partien beobachtet wurden, die zweifellos in tiefere Bodenmulden hinabreichen. W a s s e r f a r b e: Dunkelgrün. W a s s e $\mathrm{f}$ l 1 or a: Es scheinen dem See höhere Wasserpflanzen zu fehlen, hingegen erwies sich die Entwicklung der Algen als reich; in den Planktonfängen wurde auch zahlreich Phytoplankton angetroffen. W a s ser temperatur: Dieselbe betrug am 9. VIII. 1919 um $5^{\text {h }} 30^{\prime}$ p. m. an der Oberfläche $9,5-10^{\circ} \mathrm{C}$ bei einer gleichzeitigen Lufttemperatur von $12,2^{\circ} \mathrm{C}$. Temperatur der Zuflüsse in sechs Schritt Entfernung vom Seeufer $8^{\circ} \mathrm{C}$, an der Ursprungsstelle $2-3^{\circ} \mathrm{C}$. - Der See kann im Mai noch vollständig vereist sein. $\mathrm{F}$ i s c h b e s t a n d: Vermutlich Saiblinge (Salvelinus alpinus L.), in zahlreicher Anzahl. Che mische $\mathrm{Zu}$ sammensetzung des Seewassers siehe Abschnitt I u nd II (Tabelle).

Die von uns am 9. VIII. 1919 entnommenen Planktonfänge enthielten folgende Spezies:

Copepoda: Cyclops serrulatus Fischer. Cyclops vernalis Fischer.

Cla d o c e ra: Acroperus harpae BAIRD. Alona affinis (LEYDIG) var. ornata Stingelin. Bosmina coregoni. BAIRd ser. longispina RüHE. Chydorus sphaericus O. F. MïLleR.

Gmundnersee siehe Traunsee (Nr, 173). 


\section{Vorderer Gosausee.}

Fundortsnu m mer: 43. Z o n e: Nördliche Kalkalpen; Salzburger Kalkalpen. G e $0 \mathrm{gr}$ a $\mathrm{ph}$ is c h e L a g e: Oberösterreich nächst der Grenze an Salzburg, südwestlich ron Hallstadt am Fuße des Dachsteinstockes gelegen. S e eh öh e: $908 \mathrm{~m}$ ü. d. M. Flu Bge biet: Gosau-Traun-Donau. Gr ö ße: $0,53 \mathrm{~km}^{2}$ Oberfläche; $1,5 \mathrm{~km}$ lang, $0,5 \mathrm{~km}$ breit. li a $\mathrm{xi}$ mal t i e f e: $69,2 \mathrm{~m}$, mittlere Tiefe $38,2 \mathrm{~m}$.

Die spärlichen Angaben über dieses Wasserbecken stammen von Iмноғ [1885], weleher dort am 30. VIII. 1884 Planktonnetzfänge ausführte und folgende Entomostraken fand:

C o p e p o d a: Cyclops sp.

Cl a d o c e ra: Bosmina sp. Daphnia longispina O. F. MüLler.

\section{Gōsselsdorfersee.}

F u n d or t s n u m mer: 44. Z o n e: Südliche Kalkalpen; Klagenfurter Becken. Ge ograph isch e Lage: Kärnten, südlich von Völkermarkt. S e e höh e: ca. $500 \mathrm{~m}$ ü. d. M. Fl u Bg e bi et: Seebach-Drau. Gr ö ße: Ungefähr $1,4 \mathrm{~km}$ lang und $0,6 \mathrm{~km}$ breit. Tiefe: Nicht bekannt.

Über die Copepoden- und Cladocerenfauna dieses Sees hat Імноғ [1890] und Steuer [1897] berichtet; der letztere Autor hat allerdings nur die Speziesliste von Імноғ übernommen und keine eigenen Untersuchungen angestellt.

Co p e p o d a: Cyclops sp.

C l a d o c e r a: Acroperus harpae Baird [Inнof 1890 sub A. leucocephalus]. Ceriodaphnia sp. Eurycercus lamellatus (O. F. MüLler). Iliocryptus sordidus (LiÉvin). Peracantha truncata (O. F. Mï̈LleR) [Імноғ $1890 \mathrm{sub}$ Pleuroxus truncata]. Scapholeberis mucronata O. F. Mï̈LLER [ImhoF 1890 sub S. obtusa]. Sida crystallina (O. F. MüLler).

\section{Grundlsee.}

F u n d o r t s nu m mer: 45. Z o n e: Nördliche Kalkalpen; Salzkammergutgebiet der Salzburger Kalkalpen. Ge ograph is che L a ge: Nordsteiermark, nordöstlich von Aussee. S e e h öh e: $709 \mathrm{~m}$ ü. d. M. Flu Bge biet: Traun-Donau. Gr ö ße: $4 \frac{1}{2} \mathrm{~km}$ lang, ca. $1 \mathrm{~km}$ in größter Breite; 4,14 $\mathrm{km}^{2}$ Oberfläche. Mi a x i m a l t i e f e: $63,8 \mathrm{~m}$; mittlere Tiefen $33,2 \mathrm{~m}$. Ge ologisehe und hydrographisehe Daten: Nach Halbfass [1913] wird der See teilweise von Endmoränen umklammert. - Enthält nach den Unter- 
suchungen O. HaEmpeL's [i. 1.] folgende Fische: Alburnus lucidus, Alburnus mento, Cottus gobio, Lota vulgaris, Perca fluviatilis, Phoxinus laevis, Salmo salvelinus, Squalius cephalus, Trutta fario und Trutta lacustris.

Nach Imно [1885], welcher den See als erster am 29. VIII. 1884 explorierte, Keissler [1907, Besuchsdatum 11. IX. 1906] und LangHans [1911] kommen folgende Spezies ${ }^{1}$ ) bezw. Genera vor:

C o p e p o da: Canthocamptus wierzejskii Mrazek [Haempel i. 1.]. Cyclops sp. [Iмноғ 1885̃]. Cyclops fuscus Jurine [Haempel i. 1.]. Cyclops serrulatus Fischer [Haempel i. 1.]. Cyclops strenuus Fischer [Haempel i. 1.]. Cyclops viridis Jurine [Haempel i. 1.]. Diaptomus sp. [ImhoF 1885, Keissler 1907]. Diaptomus gracilis G. O. Sars [Haempel i. 1.].

Cl a d o e e r a: Acroperus angustatus G. O. Sars [Langhans 1911]. Acroperus harpae Batrd [Langhans 1911, Haempel i. 1.]. Alona costata Sars [Langhans 1911, Haempel i. 1.]. Alona guttata Sars [Langhans 1911]. Alona quadrangularis O. F. MÜLLER [Haempel i. 1.]. Alona rectangula Sars [Langhans 1911]. Alonopsis elongata Sars [Langhans 1911, Haempel i. 1.]. Anchistropus emarginatus Sars [Langhans 1911]. Bosmina sp. [IмноF 1885, KeIssler 1907]. Bosmina coregoni-longispina Leydig [Langhans 1911, Haempel i. 1.]. Bythotrephes longimanus Leydig [Imhof 1885, Langhans 1911, Haempel i. 1.]. Chydorus globosus Batrd [Langhans 1911]. Chydorus sphaericus O. F. Mülder [Haempel i. l.]. Daphnia sp. [KeIssLer 1907]. Daphnia longispina var. hyalina LeydiG [Imнof 1885 sub longispina, Langhans 1911 sub hyalina, Haempel i. I. (typica)]. Eurycercus lamellatus (O. F. MüLLER) [Langhans 1911, Haempel i. 1.]. Graptoleberis testudinaria (Fischer) [Langhans 1911, Haempel i. 1.]. Leptodora kindtii (Focke) [Imнof 1885 sub L. hyalina, Langhans 1911, Haempel i. 1.]. Peracantha truncata O. F. Müller [Haempel i. 1.]. Pleuroxus laevis G. O. Sars [Langhans 1911]. Pleuroxus uncinatus Baird [Langhans 1911, Haempel i. 1.]. Scapholeberis mucronata (O. F. Müller) [Langhans 1911, Haempel i. 1. (longicornis)]. Sida crystallina (O. F. Müller) [Langhans 1911, HaEmpel i. 1.]. Simocephalus vetulus (O. F. MülleR) [Langhans 1911, Haempel i. 1.].

1) Eine eingehende Bearbeitung des Grundlsees von O. Haempel befindet sich leider erst im Druck (Internat. Revue f. Hydrobiologie); der Autor stellte uns jedoch seine Spezieslisten (Copepoda + Cladocera) in bereitwilligster Weise zur Verfügung, so daß die von ihm nachgewiesenen Formen hier sub „HAEMPEL i. 1.“ angeführt werden konnten. - Diese Abhandlung O. HaEmpEL's ist inzwischen erschienen [Int. Rev. f. Hydrob. vol. X]. 


\section{Grũnsee.}

(Bisher nicht veröffentlicht.)

F u n d o r t s n u m me r: 46. Z o n e: Zcntralalpen; Hole Tauern. Ge ogra ph is che Lage: Salzburg, im Stubachtale (Grolglocknergebiet) gelegen. S e e h ö h e: $1699 \mathrm{~m}$ ü. d. Mi. F l u B g e b i e t: Weißenbach-Stubach-Salzach-Inn-Donau. Gr ö Be: $420 \mathrm{~m}$ lang, $315 \mathrm{~m}$ breit. Maximaltiefe: $32,2 \mathrm{~m}$. Ge ol o g is che und hydro$\mathrm{gr}$ a $\mathrm{ph}$ is ch e D a t e $\mathrm{n}$ : Das Wasserbecken liegt in einer Gneismulde. Вӧнм [1886] führt den Grünsee einerseits unter den durch Bergsturz entstandenen Stauseen an, andererseits zählt er ihn zu den durch Erosion ausgehöhlten Wannen (echte Felsbecken). Die Ufer des weiten, der vollen Besonnung ausgesetzten Sees werden, mit Ausnahme der etwas versumpften Stelle beim Einflusse des Weißenbaches am Südende, durchwegs aus steinigen Hängen gebildet; die Nordseite nimmt eine etwa $70 \mathrm{~m}$ hohe Felswand ein. An der West-, Nord- und Ostseite fällt der Seegrund ziemlich rasch ab. Außer dem Zufluß des Weißenbaches mündet in das Becken noch ein Abflußbach aus der sogenannten „schwarzen Lacke" am Nordufer und zwei kleine, wasserfallbildende Rinnsale im Westen. Nach Fugger [1899] friert der See Anfang Dezember ein und taut Mitte Mai auf. Am 22. VIII. 1920 vormittags wurde von uns eine $\mathrm{O}$ ber $\mathrm{flä} \mathrm{e} \mathrm{h}$ e $\mathrm{n}$ w a s s er t e $\mathrm{m}$ per a t u r von $7,8-8^{\circ} \mathrm{C}$ gemessen, während die Temperatur des Weißenbaches $6,3^{\circ} \mathrm{C}$ betrug (Lufttemperatur um $1^{\text {h }} 1 \check{5}^{\prime}$ p. m. $7^{0} \mathrm{C}$ ). Wasserfarbe des Spiegels intensiv hellgrün. Strömung aus der Richtung des Einflußbaches deutlich ausgeprägt. Wasserflora: Längs allen Uferrändern ist eine zahlreiche Algenvegetation entwickelt; in den Fangproben viel Phytoplankton. Fischbestand: Reich an Forellen. Che mische Zusammensetzung des Wassers siehe Abschnitt I und II (Tabelle).

Die von uns am 22. VIII. 1920 vorgenommenen Planktonnetzfänge, die vom Boote aus teils an der Oberfläche, teils in $10 \mathrm{~m}$ Tiefe ausgeführt wurden, enthielten nur folgende Formen:

Co p e p od a: Cyclops sp. (1 ठ-Expl.).

Cla docera: Acroperus harpae BAIRD. Alona affinis Leydig. Chydorus sphaericus O. F. MüLLER.

\section{Haidersee.}

Fu nd ortsnu m mer: 47. Z o n e: Zentralalpen; Rhätische Alpen, in die Grenzlinie zwischen Spölalpen- und Ötztaleralpengruppe 
fallend. Ge o g r a ph i s c h e L a ge: Tirol, Oberes Etschtal, nördlich von Glurns bei St. Valentin auf der Haid gelegen. S e e h ö h e: $1450 \mathrm{~m}$ ü. d. M. Flu Bg e biet: Etsch (Adria). Grö Be: 2,34 km lang, $0,63 \mathrm{~km}$ breit; $0,89 \mathrm{~km}^{2}$ Oberfläche. Maximalti efe: $16,5 \mathrm{~m}$, mittlere Tiefe $7 \mathrm{~m}$. Geologische und hydrographische Dat e n: Der Haidersee ist durch Schuttkegel und Murbrüche, von Wildbächen verursacht, die von beiden Seiten in das Etschtal münden, angestaut [BöHм 1886]; nach Dalla Torre [1913] wird der See hiedurch allmählich ausgefüllt werden. Er liegt im Gebiete des Gneisphyllits. Ostufer durch Schuttmassen seicht, die Tiefenzone im Westen g€legen. Der See beherbergt an F is che n: Äsche, Renke, Saibling, Seeforelle.

Über die Planktoncrustaceen des Haidersees hat ausschließlich Moniez [1887] berichtet; er konnte folgende Formen auffinden:

C o p e p o d 2: Diaptomus castor JuRIne.

Cl a d o c e r a: Alona affinis (Leydig) [sub Alona oblonga P. E. MiüLLER]. Bosmina coregoni-longispina LEydig forma dollfusi MonIEz, Daphnia longispina O. F. MüLLER.

\section{Hallstättersee.}

Fu n d ortsnu mmer: 48. Z o ne: Nördliche Kalkalpen; Salzkammergutgebiet. Ge o gra ph i s ch e Lage: Oberösterreich, bei Hallstatt am Nordfuße des Dachsteinmassivs. S e e h ö h e: $494 \mathrm{~m}$ ü. d. M. Flu Bge biet: Traun-Donau. Grö Be: $8,58 \mathrm{~km}^{2}$ Oberfläche; $8,2 \mathrm{~km}$ lang, größte Breite $2,1 \mathrm{~km}$, geringste Breite $0,3 \mathrm{~km}$. M a x i m a lt i e fe: $125,2 \mathrm{~m}$; im Mittel $64,88 \mathrm{~m}$. Ge ol o g is che und h y drographische Daten: Nach den Angaben von Haempel [1918] kommen als in den See geführte Sedimente in überwiegender Mehrheit Kalkgesteine (Dachsteinkalk, Korallenriffkalk, Muschelkalk, Kalkgruse der Gosauformation etc.) in Betracht, während dagegen schieferige und tonige Derivate zurücktreten. Bezüglich der Form stellt der Hallstättersee ein sackartiges Becken mit Steilufern dar. Außer der Traun sind vier größere konstante Zuflüsse vorhanden. Kalte und warme Quellen am Seeboden nachgewiesen. Beschaffenheit des Seegrundes: Schlammig, bis zи $8 \mathrm{~mm}$ Dicke, stark kieselsäure- und kalkhaltig. W a s s e r fl o r a in Arten höherer Wasserpflanzen (Phanerogamen) verhältnismäßig arm entwickelt, Algen und Phytoplankton reicher. Was serfarbc: Vorherrschend grün, Durchsichtigkeit gering. Te mperatur verhältnisse: Jahresmaxima für Mitte August 1911 und Mitte. 
Juli 1912 des Oberflächenwassers $17,5^{\circ} \mathrm{C}$ bezw. $18,7^{\circ} \mathrm{C}$; Jahresminima desselben für Februar 1911 und Jänner 1912 3,70 C bezw. $5^{0} \mathrm{C}$ [nach Haempel 1918]; Temperaturabnahme nach der Tiefe laut Messungen HAEMPEL'S vom 13. IX. 1913: Oberfläche $11,6^{\circ} \mathrm{C}$, in $5 \mathrm{~m} 10,7^{\circ} \mathrm{C}$, in $10 \mathrm{~m} 10,2^{\circ} \mathrm{C}$, in $20 \mathrm{~m} 10^{\circ} \mathrm{C}$, in $30 \mathrm{~m} 9,8^{\circ} \mathrm{C}$, in $40 \mathrm{~m} 8,8^{\circ} \mathrm{C}$, in $50 \mathrm{~m} 5,9^{\circ} \mathrm{C}$,

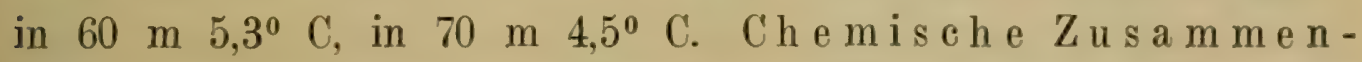
setzung des Wassers: Dominierend Calciumoxyd (51,2 mg bis 63,2 mg pro 1 Liter), Magnesium-, Natrium- und Kaliumoxyd dagegen stark zurücktretend. F is ch best a nd: Coregonus steindachneri $\mathrm{N}$., Trutta lacustris L., Salmo salvelinus L., Thymallus vulgaris N., Lota vulgaris L., Esox lucius L., Perca fluviatilis L., Cottus gobio L., Leuciscus cephalus (Неск.), Alburnus lucidus Heck., Scardinius erythrophthalmus L., Phoxinus laevis L.

Die folgende Liste von Copepoden- und Cladocerenspezies des Hallstättersees gründet sich auf die Angaben von Inнof [1885], Lorenz [1898], Keissler [1903], Brehy und Zederbauer [1906]. Langhans [1911] und Haempel [1918]; dem letzten Autor verdanken wir die eingehendste und beste Untersuchung.

Copepoda: Canthocamptus staphylinus (Jurine). [Haempel 1918]. Canthocamptus wierzejskii Míázen [Haempel 1918]. Cyclops sp. [Imhof 1885, Keissler 1903]. Cyclops fimbriatus Fischer [Haempel 1918]. Cyclops fuscus Jurine [HAEMPeL 1918]. Cyclops serrulatus Fischer [Haempel 1918]. Cyclops strenuus Fischer [Brehm und Zederbauer 1906, Haempel 1918, Lorenz 1898]. Cyclops viridis Jurine [Haempel 1918]. Diaptomus sp. [Iuhof 1885, Keissler 1903]. Diaptomus gracilis G. D. Sars [Brehy und Zederbauer 1906, Haempel 1918, LoRENz 1898].

Cl a d o e e ra: Acroperus angustatus G. O. Sars [Langhans 1911]. Acroperus harpae BAIRD [HAEMPEL 1918]. Alona affinis (LeydiG) [Langhans 1911]. Alona costata G. O. Sars [Langhans 1911]. Alona guttata G. O. Sars [Laxghans 1911]. Alona quadrangularis (O. F. Miüller) [Haempel 1918]. Bosmina sp. [Inhof 1885, Keissler 1903]. Bosmina coregoni-longispina Leydig [Lorenz 1898 sub B. bohemica Hellich, Brehy und Zederbauer 1906, Langhans 1911, Haempel 1918]. Bythotrephes longimanus Leydig [IyHof 1885, Keissler 1903, Brehur und Zederbauer 1906, Langhans 1911, Haempel 1918; Lorenz 1898]. Ceriodaphnia pulchella G. O. SaRs [HaEMPEL 1918]. Chydorus sphaericus O. F. Müller [Haempel 1918]. Daphnia sp. [Keissler 1903]. Daphnia longispina var. hyalina Leydig f. typica [Імноғ 1885 sub D. hyalina, 
Lorenz 1898 sub D. longispina var. nasuta, Brehm und Zederbauer 1906 sub hyalina, Langhans 1911 sub hyalina + longispina, Haempel 1918]. Eurycercus lamellatus O. F. Müller [Langhans 1911, Haemped 1918, Lorenz 1898]. Graptoleberis testudinaria Fischer [HAempel 1918, Langhans 1911]. Leptodora kindtii (Focke) [ІмноF 1885, Lonenz 1898, Keissler 1903, Brehm und Zederbauer 1906, Haempel 1918]. Sida crystallina O. F. Müller [Langhans 1911, HaEmpel 1918; Lorenz 1898]. Simocephalus vetulus (O. F. Mï̈lLER) [HaEmpel 1918, Lorenz 1898].

Ergänzung: Nach RüHE [1915] kommt im Hallstättersee auch Bosmina longirostris (als Litoralform) vor.

\section{Hechtsee.}

Fundorts nu m mer: 49. Z o n e: Nördliche Kalkalpen; Nordtiroler Kalkalpen. Ge o gra ph is ch e $\mathrm{Lage}$ : Nordtirol, nördlich von Kufstein. S e e höh e: $544 \mathrm{~m}$ ü. d. M. F l u ßg e b i e t: Klausenbach-Inn-Donau. Gr ö Be: $750 \mathrm{~m}$ lang, $750 \mathrm{~m}$ breit. M a x i mal tiefe: $56,5 \mathrm{~m}$. Geologische und hydrographische $\mathrm{D}$ at e $\mathrm{n}$ : Der See liegt in der Grenzlinie von Dachsteindolomit und Kößener Schichten; Ufer aus Kalkbreccie. Nach HalbFass [1913] ein Kesselbecken aus der Eiszeit. W a s s e r $\mathrm{f}$ a $\mathrm{r}$ b e: Nieergrün. W a s s e rtemperatur: Angeblich „sehr milde“; friert im Dezember zu. Enthält mehrere F is ch s p e zi es: Barsch, Koppe, Pfrille, Rotfeder, Schleie, Schmerle, Seeforelle, Steinpitzger.

BREHм [1907] meldet folgende Entomostrakengenera:

C o p e p od a: Cyclops sp. Diaptomus sp.

Cl a d o c e ra: Bosmina sp.

\section{Hintersee bei Ramsau.}

Fundortsnummer: 50. Z Z n e: Nördliche Kalkalpen; Berchtesgadnergruppe. Ge o g r a $\mathrm{ph}$ i s c h e L a g e: Südbayern, westlich von Berchtesgaden, bei Ramsau. S e eh öh e: $732 \mathrm{~m}$ ü. d. M. Flu Bg e bi et: Almache-Salzach-Inn-Donau. Größ e: $850 \mathrm{~m}$ lang, $500 \mathrm{~m}$ breit. M a xi ma l ti e f e: Unbekannt. Ge olog is e h e und hydrographische Daten: Nach einer Messung von BRенм [1906] betrug die oberflächliche Wassertemperatur am 15. VIII. 1904 ungefähr $18^{\circ}$ C. - Der See soll reich an Saiblingen sein.

BREнm [1906] konstatierte das Vorkommen folgender Spezies:

Co p e p o d a: Cyclops strenuus Fischer. 
$\mathrm{Cl}$ a d o e c r a: Acroperus angustatus SARS. Bosmina coregoni BaIRD. Daphnia longispina var. hyalina LEYDIG.

\section{Hintersteinersee.}

Fun d ortsn u m mer: 51. Z o ne: Nördliche Kalkalpen; Nordtiroler Kalkalpen; Kaisergebirge. G e 0 gr a $\mathrm{ph}$ is c h e L a g e: Nordtirol, südöstlich von Kufstein. S e ehöh €: $892 \mathrm{~m}$ ü. d. M. F lu Bg e bi et: Seebach-Weißachbach-Inn-Donau. Größe: $1200 \mathrm{~m}$ lang, $500 \mathrm{~m}$ breit. Maximaltiefe: $35,6 \mathrm{~m}$. Ge ol og is che u nd h ydrograph is che Dat en: Der See liegt im Triasdolomit (Partnachdolomit); wird hauptsächlich aus Quellen am Nordufer gespeist. Nach HalbFass [1913] gehört der Hintersteinersee zu den Felsbecken. Wasser klar; in den seichteren Teilen blaßgr ün, in den tieferen azurblau gefärbt. Enthält Forellen.

Auffälligerweise meldet BreHM [1907] eine e in zige Cla do cerenspezies aus dem See, Copepoden überhaupt keine; eine Nachuntersuchung wäre daher dringend nötig.

Cl a d o e e r a: Alonella nana (BAIRD).

Hochalpensee siehe Kreuzjochsee (Nr.68).

\section{Hopfensee.}

Fu n d o r ts n u m mer: 52. Z o ne: Nördliche Kalkalpen; Voralpen der Vilsergebirgszone. Ge ograph is che Lage: Bayern, nördlich von Füßen. S e eh öh e: $785 \mathrm{~m}$ ü. d. M. Flu Bgebiet: Lech-Donau. Größc: Im Durchmesser ungefähr $2,3 \mathrm{~km}$ breit. $T$ i e f c : Nicht ermittelt.

Імноғ [1887], der in den Monaten August und September Planktonnetrfänge ausführte, fand in diesem Wasserbecken folgende Spezies und Genera:

Co pe p o da: Cyclops sp. Diaptomus sp.

Cl a d ocera: Bosmina sp. Daphnia longispina var. cucullata SARS forma kahlbergensis (SCHOEDLER). Diaphanosoma brachyurum (LiÉviv). Leptodora kindtii (FocKE).

\section{Idrosee (Lago d'Idro).}

Fu n d ortsnummer: 53. Z o ne: Südliche Kalkalpen; Etschbuchtgebirge. Ge ographische Lage: Italien, Bezirk Brescia, westlich vom Gardasee, nächst der Tiroler Grenze. S e e h öh c: $378 \mathrm{~m}$ 
ï. d. M. F 1 u g g e b i e t: Chiese-Oglio-Po. Gr ö B e: $9,6 \mathrm{~km}$ lang, $1,9 \mathrm{~km}$ breit; $14000 \mathrm{~m}^{2}$ Oberfläche. M a x i m a l t i e f e: $122 \mathrm{~m}($ ? $300 \mathrm{~m})$.

Nach den Untersuchungen von Pavesi [1879] findet sich folgendes Crustaceenplankton:

Co pe poda: Cyclops albidus Jurine (sub C. tenuicornis CL.). Cyclops diaphanus Fischer (sub C. minutus CL.).

C l a d o e e r a: Daphnia longispina var. cristata G. O. SARs (sub D. cristata S.). Daphnia pulex (DE GEER) (sub D. magna STR.). Leptodora kindtii (FocKE).

\section{Iseosee (Lago d'Iseo).}

Fundorts num mer: 54. Z o ne: Südliche Kalkalpen; Etsclbuchtgebirge. Ge $0 \mathrm{graph}$ is ch e Lage: Italien, Bezirk Bergamo, Brescianer Alpen. Seehöhe: $192 \mathrm{~m}$ ü. d. M. Flu Bgebiet: Oglio-Po. Gr öße: $60000 \mathrm{~m}^{2}$ Oberfläche; $15 \mathrm{~km}$ lang, $4,6 \mathrm{~km}$ breit. Maximaltief e: $260 \mathrm{~m}(340 \mathrm{~m}$ ?). Ge ol o g is che und h y d c cgraphis ehe Daten: Uns nicht bekannt.

Die folgende Speziesliste gründet sich auf die Untersuchungen von Pavesi [1879].

Co p e p o da: Cyclops albidus Jurine (sub C. tenuicornis Claus). Cyclops diaphanus Fischer (sub C. minutus Claus). Diaptomus gracilis G. O. SARS.

Cl a d o c e ra: Bythotrephes longimanus (LEYDIG). Ceriodaphnia quadrangula (0. F. Müller). Daphnia longispina O. F. Müller. Daphnia pulex (DE GEER). Diaphonosoma brachyurum (LIÉvIN). Leptodora kindtii (Focke).

\section{Jeserzsee (Jeserzersee).}

F u n d o r t s n u m mer: 55. Z o n e: Südliche Kalkalpen; Klagerfurter Becken. Ge o gra ph i s e h e Lage: Kärnten, nördlich vom Wörthersee, bei Kranzelhofen nördlich von Velden. Se ehöh e: ca. $620 \mathrm{~m}$ ü. d. M. F I u B g e b i e t: Drau. Gr ö B e: Etwa $550 \mathrm{~m}$ lang und $280 \mathrm{~m}$ breit. Tiefe: Unbekannt.

Über die Cladoceren- und Copepodenfauna des Jeserzersees haben Imhof [1890], Steuer [1897, Angaben ex Imhof] und Keissler [1906] berichtet; demnach beherbergt er folgende Formen:

Co p e p o da: Cyclops sp. [ImhoF 1890, Steuer 1897, Keissler 1906]. Diaptomus sp. [Keissler 1906].

Cl a d o c e r a: Alona costata G. O. SARS [Imhof 1890, Steuer 1897]. Alona guttata G. O. Sars [Imhof 1890, Steuer 1897]. Bosmina sp. 
[Imhof, Steuer, Keissler]. Camptocercus rectirostris (Schoedler) [Iмноғ, Steuer). Ceriodaphnia sp. [Imнof, Steuer]. Chydorus sp. [Iмноғ, Steuer]. Diaphanosoma brachyurum (Liévin) [Iмноғ, Steuer]. Graptoleberis testudinaria (Fischer) [Iмноғ, Steder] (sub Alona test.). Latona setifera (O. F. Müller) [Imhof, Stever]. Peracantha truncata (O. F. Müller) [Imhof, Steuer] (sub Pleuroxus tr.). Sida crystallina (O. F. MIüller) [Imhof, Stever]. Simocephalus vetulus (O. F. Müller) [ІмноF, Steuer].

\section{Igelsee beim Attersee.}

F u n d o r t s n u m mer: 56. Z o ne: Nördliche Kalkalpen; Salzkammergut. Ge ographische Lage: Beim Südwestende des Attersees am Fuße des Hollerberges gelegen; Oberösterreich. Se e höh e: $621 \mathrm{~m}$ ü. d. M. F.lu Bge biet: Ager-Traun-Donau. Gr ö Be: Kleines Becken von etwa $120 \mathrm{~m}$ im Höchstausmaße.

Langhans [1911] führt folgende Cla d o c e re $\mathrm{n}$ für diesen See an:

Acroperus harpae (BAIRD). Camptocercus lilljeborgi SchoEdLer. Camptocercus rectirostris var. biserratus SchoEdLer. Ceriodaphnia pulchella G. O. SARs. Ceriodaphnia quadrangula var. hamata LiLlJ. (sub C. wiülleri var. hamata). Graptoleberis testudinaria (FISCHER). Lathonura rectirostris (0. F. Mİ̈LLER்). Peracantha truncata (O. F. Müller). Scapholeberis mucronata (O. F. MüLler). Sida crystallina (O. F. Müller). Simocephalus vetulus (O. F. MüLLER).

\section{Kalterersee.}

Fu nd ortsnu mmer: 57. Z o ne: Südliche Kalkalpen; Etschbuchtgebirge; Nonsberg. Ge $0 \mathrm{gr}$ a $\mathrm{ph}$ is c h e L a g e: Südtirol, südlich von Kaltern, im Gebiete des Nionte Roën. S e e h ö h e: 216 m ü. d. M. F l u B g e b i et: Etsch (Adria). Gr ö Be: ca. 140 ha Oberfläche; $2 \mathrm{~km}$ lang, $1 \mathrm{~km}$ breit. Maximaltiefe: $5-6 \mathrm{~m}$. Ge ologisehe und hydrographische Daten: Liegt als flacher Wannensee im Alluvialschutt der Etsch angestaut. Ufer stark versumpft; Wasser schmutzig, von hellgrüner Färbung. Als W a s s e r t e mper a t u r an der Oberfläche wurde in den Monaten Juli und August 1902 von Huber [1907] 23,8-25,70 C abgemessen; Mitte Dezember fror der See zu. Enthält an Fisch en: Aal, Barsch, Hecht, Karpfen, Nase und Schleie.

Nach den eingehenden Untersuchungen von Huber [1907], welcher seine Beobachtungen durch mehrere Monate (Juni bis November des 
Jahres 1902) fortsetzte, setzt sich die Copepoden- und Cladocerenfauna des Kalterersees aus folgenden Spezies zusammen:

Copepoda: Cyclops insignis Craus (?). Cyclops leuckarti Claus.

Cla d o cera: Bosmina coregoni-longispina (Leydig). Bosmina longirostris-cornuta (O. F. MüLLER). Ceriodaphnia sp. Chydorus sphaericus (O. F. MülLER). Daphnia longispina var. hyalina Leydig. Diaphanosoma brachyurum (LiÉvin). Leptodora kindtii (Focke). Pleuroxus sp.

Kammersee siehe Attersee (Nr. 9).

Kammersee bei Aussee.

Fu n d ortsnu m mer: 58. Z o ne: Nördliche Kalkalpen; Salzburger Kalkalpen, Gebiet des Salzkammergutes. Ge o g r a ph i s c h e L a ge: Steiermark, Bezirk Aussee, westlich vom Toplitzsee gelegen. S e e h ö h e: 720 m ü. d. M. Fl u Bg e b i e t: Gößlbach-Traun-Donau. Gr ö B e: $330 \mathrm{~m}$ lang und $140 \mathrm{~m}$ breit; Oberfläche $0,04 \mathrm{~km}^{2}$. Tiefe: $7 \mathrm{~m}$ [MüLlner 1896].

Von Langhans [1911] werden für diesen kleinen See folgende Cladoceren erwähnt:

Cl a d o cer a: Acroperus harpae BaIRD s. str. (sub A. bairdi). Acroperus harpae var. frigida Ekman. Alona affinis (Leydig). Alona quadrangularis (0. F. MüLLER). Polyphemus pediculus (LinnÉ).

\section{Kampspitzsee.}

(Bisher nicht veröffentlicht.)

F u n d o r t s n u m mer: 59. Z o n e: Zentralalpen; Niedere Tauern. Ge $0 \mathrm{graph}$ is ch e Lage: Obersteiermark, südlich von Schladming, im Gebiete der Giglachseen. S e e h öh e: ca. $2200 \mathrm{~m}$ ü. d. M. Flu Bg e bi e t: Giglachbach-Talbach-Enns-Donau. Gr ö B e: Ungefähr $300(225 \mathrm{~m})$ Schritte lang und höchstens halb so breit. Ma x i ma lt i e f e: Nicht ermittelt, vermutlich nicht erheblich; in 3-4 m Entfernung vom Ufer wurden von uns Tiefen von höchstens $3 \mathrm{~m}$ konstatiert, der Seegrund ist überall sichtbar. Ge ol o g i s che und hydrographis che Daten: Das Becken liegt im Urgestein. Ufer nur an der Süd- und Südostseite aus gerundeten Kuppen gebildet, sonst steil geneigte Geröllhalden längs des Fußes der Kampspitze (2400 m). Bodenbeschaffenheit glimmerig-sandig. W a s s e r f 1 o r a makroskopisch fast gar nicht entwickelt, spärliche Klümpchenalgen an Steinen in der 
Nähe des Abflusses. Als oberflächliche Was s ert e mper a tur wurde von uns am Besuchstage (10. VIII. 1919) nur 1,30 $\mathrm{C}$ abgemessen; der See war über die Hälfte seines Areals vereist. (Gleichzeitige Lufttemperatur im Schatten betrug $10^{\circ} \mathrm{C}$.) Der See enthält k e i n e F is c h e, nur Nematoden, Ostracoden und einige Insektenlarven wurden bcobachtet. Chemische Zusammensetzung des Wassers siehe Absehnitt I und II (Tabelle).

Im Inhalt der Planktonnetzfänge konstatierten wir folgende Spezies:

Co p e poda: Cyclops vernalis Fischer. Canthocamptus schmeili var. biserialis Micoletzky.

Cl a d o c e ra: Alonella nana (BAIRD).

\section{Keutschachersee (= Plaschischensee).}

F u n d orts n u m mer: 60. Z o n e: Südliche Kalkalpen; Klagenfurter Becken. Ge ographis ch e Lage: Kärnten, südlich vom Wörthersee. S e e h öh e: $508 \mathrm{~m}$ ü. d. M. Flu Bge bi e t: Reifnitzerbach-Wörthersee-Drau. Gr ö Be: 1,4 kn² Oberfläche; 2,1 km lang, $1,1 \mathrm{~km}$ breit. Maximaltiefe: $15 \mathrm{~m}$. Ge ologische und h ydrographische Daten: Nach Richter [1897] liegt das. Becken in einem Tale, das sich an der Grenze der Schieferberge und einem tertiären, dem Karbon aufgelagerten Hügellande hinzieht.

IмноF [1890], Steuer [1897], welcher diesen See am 12. September 1896 besuchte, und Keissler [1906], welcher dort am 5. Juli 1905 Planktonfänge ausführte, fanden folgende Formen:

Co p e p o da: Cyclops sp. [Keissler 1906, Imhof 1890)]. Cyclops. leuckarti Claus [Steuer 1897].

Cl a d o e e r a: Alona affinis Leydig. [Steuer 189i]. Bosmina sp. [KeISSLER 1906; ImhoF 18901]. Bosmina coregoni-longispina LeYdig forma bohemica Sting. [Steuer 1897]. Chydorus latus G. O. Sars [Steuer 1897], Daphnia sp. [KeIssler 1906]. Daphnia longispina var. cristata SARS

1) Außerdem gibt Iмноғ [1890] für diesen Fundort noch 5 Formen an, die von den späteren Autoren nicht mehr selbst beobachtet wurden; es sind dies: Ceriodaphnia sp. Daphnia longispina var. hyalina LEydig. Daphnia longispina var. cucullata SARS f. Kahlbergensis SchOEDlER. Diaphanosoma brachyurum (LiÉvin). Leptodora kindlio (FOCKE).

Anmerkung: Es ist zweifellos auffallend, daß in diesem See nicht weniger als 3 verschiedene Variationsformen der Daphnia longispina gleichzeitig vorkommen sollen; der Fall würde einer Revision bedürfen. STEUER [189i] hat die Angaben Імноғ's in seine Arbeit übernommen. 
forma cederstrëmii Schoedler [STEuer 1897 sub D. cederstrëmii]. Latona setifera (O. F. Müller) [Steuer 1897]. Sida crystallina (O. F. NiülLer) [StEuER 1897 und IмноF 1890].

\section{Oberer Klaftersee.}

(Bisher nicht veröffentlicht.)

F u n d o r t s n u m mer: 61 . Z o n e: Zentralalpen; Niedere Tauern. Geographische Lage: Obersteiermark, bei St. Nikolai im Sölktale. S e e h ö h e: ca. $2000 \mathrm{~m}$ ü. d. M. F l u Bg e b i e t: Hohenseebach-Große Sölk-Enns-Donau. G r ö ß e: Ungefähr 300 (225 m) Schritte lang. Maximaltiefe: Vermutlich ein seichtes Becken ohne Tiefen über $10 \mathrm{~m}$. Ge o logis ch e und h y drographische $\mathrm{D}$ a t e n: Der See liegt im Glimmerschiefer. Seine Ufer sind nirgends steil, große Strecken des Nordwestrandes sind versumpft. Der Seegrund zeigt feinsandige und lehmige Beschaffenheit. F är bu ng des W a s s e r s: Dunkelgrün. W a s s e r t e m p e r a t u r: Dieselbe betrug an der Oberfläche $7^{\circ} \mathrm{C}$, jene eines der zwei stärkeren Zuflüsse $5,8^{\circ} \mathrm{C}$ (am 7. VIII. 1919 gemessen). Wasserflora: Schilfbestände und submerse Phanerogamen, sowie die Algenvegetation reich entwickelt; Phytoplankton, insbesondere Desmidiaceen, in den Proben stark vertreten.

Unsere Planktonnetzfänge enthielten folgende Spezies:

C o p e p o d a: Cyclops vernalis Fischer.

Cla do e e ra: Alona affinis Leydig var. ornata Sting. Alonella excisa (Fischer). Chydorus sphaericus O. F. Mǘlen, Type A.

\section{Klammjochsee.}

(Bisher nicht veröffentlicht.)

F u n d o r t s n u m mex: 62. Z o n e: Zentralalpen; Tuxer Schieferalpen. Ge o graphis che Lage: Tirol, am Übergang (Joch) vom Wattental in das Navistal gelegen. S e e h ö h e: $2350 \mathrm{~m}$ ü. d. M. F l u Bg e b i e t: Der See hat keinen sichtbaren Zu- oder Abfluß; sein Niederschlagsgebiet gehört zum Navisbach-Sill-Inn-Donau. Grö Be: Kleines Hochgebirgsbecken von ungefähr $100 \mathrm{~m}$ Länge und $60 \mathrm{~m}$ Breite Geologische und hydrographische Daten: Liegt im Tonschiefergebiet. Der Kammjochsee gehört zu den ausgesprochenen Flachseetypen mit niedrigen, nur sanft ansteigenden, begrasten Uferrändern und wenig geneigtem Seegrund. Letzterer ist von schlammiger Beschaffenheit. W a s s e r f är b u ng: Grün. W a s ser t e m perat u r: Dieselbe betrug um $6 \mathrm{~h}$ p. m. am 8. VIII. 1921 nicht weniger als 
$17,6^{\circ} \mathrm{C}$, was auf die geringe Tiefe und volle Wirkung der täglichen Besonnung hindeutet (gleichzeitig gemessene Lufttemperatur $\left.15,5^{\circ} \quad \mathrm{C}^{\prime}\right)$. Die makroskopische Algenflora ist sehr gering ent wickelt, höhere Wasserpflanzen fehlen vollständig.

Die am 8. VIII. 1921 dem See entnommenen Planktonnetzfangproben enthielten folgende Formen:

Co p e p o d a: Cyclops strenuus Fischer.

Clad o c era: Alonella excisa (Fischer). Chydorus sphaericus O. F. Müller. Daphnia longispina var. longispina s. str. f. rosea-carifrons SARS.

Mittlerer Klippensee im Klafferkessel.

(Bisher nicht veröffentlicht.)

F u n d o r t s n u m me r: 63. Z o n e: Zentralalpen; Niedere Tauern. Ge $0 \mathrm{gr}$ a $\mathrm{ph}$ is ch e Lage: Obersteiermark, südlich von Schladming im Gebiete des sogenannten Klafferkessels (Waldhornspitze $2706 \mathrm{~m}$ ) gelegen. S e ehöh e: $2257 \mathrm{~m}$ ü. d. M. Flu Bge bi et: Steinwiesenbach-Talbach-Enns-Donau. Gr ö ße: Der größte Durchmesser des rundlich geformten Hochgebirgssees beträgt kaum $100 \mathrm{~m}$. M a x j m a lt i efe: Dieselbe dürfte nach unseren Beobachtungen 3-4 m nicht übersteigen. Ge ologis che und hydrographische Daten: Das Gestein des gesamten Klafferkessels besteht aus Glimmerschiefer. Seine Bildung sowohl wie die seiner zahlreichen Wasserbecken (ca. 30!) geht zweifellos auf die Tätigkeit eines Gletschers zurück, dessen letzter Rest noch heute in einem kleinen Eisfelde des Rauhenbergkaares erhalten ist [vergl. hiezu H. WöDL: „Der Klafferkessel in den Schladmingeralpen“, Zeitschr. d. D.Ö.A.V., vol. 49, 1918, p. 101-124]. Die Umrandung des Sees wird durchwegs von niedrigen Felsriegeln gebildet, so daß die ganze flache Bodenmulde einer vollständigen Besonnung ausgesetzt ist. Trotzdem lagerten am Tage unserer Untersuchung (18. VIII. 1919) ausgedehnte Schneefelder am Nordost-, Südost- und Südufer des Sees und war der Spiegel selbst auf weite Strecken mit Eis bedeckt, während große abgeschmolzene Eistrümmer als Schollen umherschwammen. Den mittleren Klippensee trennt vom unteren Klippensee nur eine sehr schmale und niedere Bodenwelle ab; ein kurzes, kleines Rinnsal vermittelt die Velbindung. Als $\mathrm{Z}$ u f̊l üs s e wurden von uns drei kleine Bäche beobachtet, von denen zwei dem Ostufer, einer dem Nordzipfel des Sees unterhalb der unteren Klafferseharte angehören. Die Absenkung des Seebodens gegen die Mitte, wo sich eine kleine Inselbank erhebt, ist äußerst schwach; gro be Seichtwasserflä che n herrschen daher vor. Der Sec- 
boden erscheint von Platten des Schiefergesteines bedeckt, zwischen welchen er überall sandartige Beschaffenheit zeigt. Wasser klar, von dunkelgrüner Färbung. Als o b e r f l ä c h l i c h e W a s s e r t e m p e r at u r maßen wir $3-5^{0} \mathrm{C}$ bei gleichzeitiger Lufttemperatur von $10,2^{\circ} \mathrm{C}$ im Schatten (am 18. VIII. 1919). Eine makroskopische Wasserflora ist - mit Ausnahme eines schwachen, dunkelgefärbten Algenbelages an Steinen - nicht entwickelt; hingegen ergab die Untersuchung des Netzplanktons das Vorhandensein von Planktonalgen. - Fische wurden nicht beobachtet. - Chemisehe $\mathrm{Zus}$ ammensetzung des Seewassers siehe Abschnitt I und II (Tabelle).

Die Untersuchung der Planktonfänge ergab das Vorkommen folgender Spezics:

Co p e p o da: Canthocamptus schmeili Mrazek. Cyclops serrulatus Fischer. Cyclops vernalis Fischer.

Cla d o c era: Acroperus harpae (BAIRD). Chydorus sphaericus O. F. Müller.

\section{Klopeinersee.}

F u n d o r t s n u m m er: 64. Z o n e: Südliche Kalkalpen; Klagenfurterbecken. Ge o graph i s ch e L a g e: Kärnten, bei Völkermarkt. S e e höh e: 448 m ü. d. M. Flu Bg e bi e t: Hat keinen sichtbaren $\mathrm{Zu}$ - oder Abfluß; gehört zum Gebiete der Drau. Gr ö B e: 1,125 km² Oberfläche; $2,1 \mathrm{~km}$ lang, $0,8 \mathrm{~km}$ breit. Maximaltiefe: $48 \mathrm{~m}$. Ge ologische und h y r o graphise he Daten: Nach RICHTER [1897] liegt der See in einer Bucht des tertiären Hügellandes, welches die Südhälfte des Klagenfurter Beckens eriüllt; ein Stausee durch Sehotter.

Nach Імноғ [1890], Steuer [1897 mit Angaben ex Iмноғ] und Keissler [1906] enthält der Klopeinersee folgende Formen:

Co p e p oda: Cyclops sp. [Imhof, Steuer, Keissler]. Diaptomus sp. [Imhof, Steuer, Keissler].

Cla d o e e ra: Acroperus harpae Baird [Imhof, Steuer; sub A. leucocephalus]. Bosmina sp. [Imhof, Steder, Keissler]. Campto. cercus rectirostris (Schoedler) [Imноғ, Steuer]. Ceriodaphnia sp. [Iмноғ, Steuer]. Daphnia ip. [Keissler]. Daphnia longispina var cucullata SARS f. kahlbergensis Schoedler [Imhof, Steuer]. Daphnia longispina var. hyalina LEYdig [I, MHоF, STEUER]. Diaphanosoma brachy. urum (LiÉvin) [Імноғ, Steuer]. Leptodora kindtii (Focke) [Імноғ, StEuer]. Scapholeberis mucronata (O. F. MüLler) f. obtusa [ІмноF, Steuer]. Sida crystallina (O. F. Mülder) [Imhof, Steuer]. 


\section{Königssee.}

Fundortsnummer: 65. Z Z ne: Nördliche Kalkalpen; Berchtesgadnergruppe der Salzburger Kalkalpenzone. Ge og r a ph is che L a g e: Bayern, südlich von Berchtesgaden. S e e h ö h e: $603 \mathrm{~m}$ ü. d. M. Flu Bge bi et: Ache-Salzach-Inn-Donau. Grö Be: $8 \mathrm{~km}$ lang, $2 \mathrm{~km}$ breit. Maximaltiefe: $190 \mathrm{~m}$. Geologische und h ydrographis che Dat en: Dieser Alpensee ist durch seine steil abfallenden Ufer (Kalkfelswände) bekannt. Nach HalbFass [1913] kann er als übertieftes Gletscherbett (РЕNсK) oder als ein durch tektonische Verwerfungen entstandener See (Böse) gedeutet werden. Geistbeck [1885] gibt für den 19. IX. 1873 cine Oberflächenwassertemperatur von $12,2^{\circ} \mathrm{C}$ an. - Enthält Alpenforellen.

Imhof [1887], Hofer [1896] und Brehi [1906] melden alis dem Königssee folgende Genera und Spezies:

Copepoda: Cyclops sp. [IмноF]. Cyclops strenuus Fischer [Hofer, Brehm]. Diaptomus sp. [Iмноғ]. Diaptomus bacillifer Koelbel [Brehm]. Diaptomus gracilis G. O. Sars [Hofer].

Cl a d o c e ra: Acroperus angustatus Sars [BReHm]. Bosmina sp. [IмHoF]. Bosmina coregoni-longispina LEYDIG [HoFER sub B. longispina, BReHM sub B. coregoni]. Ceriodaphnia quadrangula var. hamata LiluJ. [BReHi]. Chydorus sp. "vermutlich sphaericus O.F. Müllen") [BReHм]. Daphnia sp. [Iмноғ]. Daphnia longispina var. hyalina Leydig [HoFER, Brenm; sub D. hyalina]. Peracantha truncata (O. F. Müller) [Brehm]. Pleuroxus aduncus (Jurine) [BReHm]. Simocephalus vetulus (O. F. MÜLLER) [BREHM].

\section{Königsangersee.}

(Bisher nicht veröffentlicht.)

F u n d o r t s n u m mer: 66. Z o ne: Zentralalpen; Hohe Tauern. Ge $0 \mathrm{graph}$ is ch e L a ge: Kärnten, Bezirk Gmünd-Sachsenburg, im Gebiete der Hohen Leier nördlich des Mölltales gelegen. S e e h ö h e: $2173 \mathrm{~m}$ ü. d. M. F l u B g e b i e t: Reinitzbach-Lieser-Drau. G r ö B e: Kleiner Hochgebirgssee von langgestreckter Gestalt mit ungefähr $200 \mathrm{~m}$ Länge und $50 \mathrm{~m}$ Breite. M a x i ma $\mathrm{t}_{\mathrm{i}} \mathrm{e}$ e: Nicht ermittelt, jedoch schätzungsweise nur einige Meter. Ge ologie der Umgebung: Liegt im Urgestein.

Die uns von Regierungsrat Dr. A. Penther übermittelten Planktonnetzfänge, welche vom 17 . VIII. $192112^{\mathrm{h}} 15^{\mathrm{\prime}}$ mittags stammen und vorwiegend Phytoplankton enthielten, wurden vor ihrer endgültigen 
Bearbeitung leider vernichtet, so daß wir hier nur mehr die bereits konstatierten Genera anführen können.

Co pe po da: Canthocamptus sp. Cyclops sp.

Cl a d o c e ra: Acroperus sp. Alona sp. Chydorus sp. Daphnia sp.

\section{Kreuthsee.}

Fundortsnu mmer: 67. Z o n e: Nördliche Kalkalpen; Nordtiroler Kalkalpenzone. G e o g r a ph i s c h e L a g e: Südbayern, südlich vom Tegernsee bei Bad Kreuth. S e e h ö h e: $800 \mathrm{~m}$ ü. d. M. Fl u Bg e b i e t: Weißachbach-Mangfall-Inn-Donau. Gr ö B e und übrige Daten unbekannt.

Ủber dieses Wasserbecken, über welches uns keine näheren Angaben zur Verfügung stehen, hat RICHARD [1896] berichtet; er zählt folgende Spezies auf:

Co p e p o d a: Cyclops fuscus (Jurine). Cyclops serrulatus Fischer.

Cla d o cera: Alona affinis Leydig. Chydorus sphaericus O. F. MÜlLer. Pleuroxus aduncus (Jurine).

\section{Kreuzjochsee (= Hochalpensee).}

Fu nd ortsnummer: 68. Z o n e: Südliche Kalkalpen; Südtiroler Dolomiten. Ge ograph is che Lage: Südtirol, östlich von St. Vigil im Enneberg, am Kreuzjoch gelegen. S e e h ö h e: ca. $2200 \mathrm{~m}$ ü. d. M. Fl u B g e b i e t: Vigilbach-Gader-Rienz-Eisack-Etsch. G r ö B e: Hochgebirgssee von 300 (225 m) Schritten Länge und 100 (75 m). Schritten Breite. Maximalti efe: Einige Meter, Ufertiefen bis zu $4 \mathrm{~m}$ gemessen. Ge ologis che und hydrographische Dat e n: Das Seebecken liegt im Wegener Dolomit [BlaAs 1902]. Ufer im Norden steilere, begraste Kuppen, im Westen und Osten flach und versumpft; sichtbare $\mathrm{Zu}$ - und Abflüsse fehlen. Seegrund wenig steinig, vorherrschend sandig und schlammig. Als oberflächliche Wassert e m peratur wurde von uns am 10. VII. 1913 in der Zeit von $1^{\text {h }} 30$ bis $3^{\text {h }}$ p. m. 9,45-9,70 C gemessen (gleichzeitige Lufttemperatur $7,7^{\circ} \mathrm{C}$ ). Makroskopische Wasserflora reich entwickelt, ebenso reichlich Phytoplankton vorhanden. Färbung des Wassers: Dunkelgrün. F is ch bestand: Phoxinus phoxinus (L.) zahlreich vorkommend. Che mische Zusammensetzung des Seewassers siehe A bs ch nitt II (Tabelle).

Aus den Planktonnetzfängen wurden von Pesta [1914] folgende Spezies determiniert: 
C o p e p o da: Cyclops serrulatus Fischer.

Cla do cera: Alona affinis Leydig. Alona affinis var. ornata Stingelin. Alona rectangula G. O. Sars. Alonella excisa (Fischer).

\section{Kröllensee ( $=$ Steinsee).}

Fundortsnummer: 69. Z o ne: Nördliche Kalkalpen; Salzkammergut. Geographisehe Lage: Salzburg, südlich vom Mondsee im Schafberggebiet. S e ehöh e, sowie alle übrigen Daten unbekannt; es handelt sich zweifellos um ein sehr kleines Wasserbecken, welches auf den Karten nicht eingezeichnet ist (MüLlNER [1896] gibt diesbezüglich Aufklärung, da er sagt: „Der dritte See der Gipfelregion des Schafberges, der Kröllensee - Hintersee der Spezialkarte - ist eine seichte, häufig austrocknende Wanne") und nur in der Bearbeitung von LANGHans [1911] erwähnt wird, welcher für diese Lokalität eine einzige Spezies anführt, nämlich:

Alona quadrangularis (O. F. MüLler).

\section{Krottensee (Krotensee).}

Fundortsnummer: 70. Z o ne: Nördliche Kalkalpen; Salzkammergut. Ge o gr a ph i s e h e L a g e: Salzburg, bei Schloß Hüttenstein am Fuße des Schafberges. S e eh öh e: 590 m ü. d. M. Flußg e b i e t: Kösselbach-Ischl-Traun-Donau. Gr ö B e: ca. $390 \mathrm{~m}$ im Durchmesser. Maximaltiefe: $45,5 \mathrm{~m}$. Ge $0 \mathrm{log}$ is che und hydrographische Daten: Unbekannt; dürfte ein Kesselbecken sein.

Die Entomostrakenfauna dieses Sees wurde von Inнof [1885]. Brehm und Zederbauer [1906] und von Langhans [1911] ${ }^{1}$ ) untersucht und folgende Spezies nachgewiesen:

Copepoda: Cyclops sp. [Імноғ 1885]. Diaptomus sp. [Імноғ. 1885]. Diaptomus gracilis G. O. Sars [Brehm und Zederbauer 1906].

C l a d o e e r a: Bosmina sp. [Імноғ 1885]. Bythotrephes longimanus Leydig [Imнof 1885]. Ceriodaphnia laticaudata P. E. Müller [Langhans 1911]. Ceriodaphnia reticulata (Jurine). [Langhans 1911]. Daphnia sp. [Iмно 1885]. Daphnia longispina var. cucullata SARs f. kahlbergensis Schoedler [IмноF 1885 sub $D$. kahlbergensis]. Daphnia

1) Die von Langhans untersuchte Probe trug die Etikettierung „Krottensee bei Gmunden"; da ein solcher See bei Gmunden nicht existiert, kann sich die Bezeichnung nur auf eine irrtümliche Beschriftung zurüchführen und als Fundort nur der Krottensee am Fuße des Schafberges bei Schloß Hüttenstein gemeint sein. 
longispina var. hyalina Leydig [ІмноF 1885 sub D. longispina, Brenм und Zederbauer 1906 sub D. hyalina]. Diaphanosoma brachyurum (LuÉvin) [Імноғ 1885]. Leptodora kindtii (Focke) [Імноғ 1885].

\section{Krummsee ').}

F u n d orts nu mmer: 71. Z o n e: Nördliche Kalkalpen; Nordtiroler Kalkalpen. Ge og r a ph is ch e La ge: Nordtirol, bei Kramsach im Unterinntale. Se ehöh e: $557 \mathrm{~m}$ ü. d. M. Flu Bge biet: Achenrainerbach-Brandenbergerache-Inn-Donau. Gr ö Be: 4,9 ha Oberfläche; $350 \mathrm{~m}$ lang, $150 \mathrm{~m}$ breit. M a x i m a lt i e f e: $2,5 \mathrm{~m}$. G e 0 logische und hydrographische Daten: Nach BlaAs [1902] liegen alle Reintalerseen, wozu auch der Krummsee gehört, in Glazialschutt, welcher eine Decke über Dolomit und Tertiärschichten bildet. Erreicht im August nach Brenм [1907] eine W as sertemperatur bis zu $23^{\circ}$ C. Wasserflora reich entwickelt. Enthält Fische.

Von BREнm [1907] werden folgende Spezies aufgezählt ${ }^{1}$ ):

Co p e p od a: Cyclops sp. (invenis). Cyclops macrurus G. O. SARS.

Cl a d o c e r a: Ceriodaphnia pulchella G. O. SARs. Diaphanosoma brachyurum (LIÉvin). Graptoleberis testudinaria (FIscher). Peracantha truncata (O. F. MÜLLER). Scapholeberis mucronata (O. F. MüLleR). Sida crystallina (O. F. MÜLLER).

\section{Lagoraisee (= Lago grande di Lagorai).}

Fundortsnummer: 72. Z o ne: Südliche Kalkalpen; Südtiroler Dolomitengebiet. G e $0 \mathrm{~g} \mathrm{r}$ a $\mathrm{ph}$ is c h e L a g e: Südtirol, südlich von Cavalese im Gebiete der Cima delle Stellune. S e e h ö h e: $1868 \mathrm{~m}$ ü. d. M. Flu B g e bi et: Lagoraitalbach-Avisio-Etsch. Gr ö be: $700 \mathrm{~m}$ lang, $250 \mathrm{~m}$ breit. M a x i mal t i e f e: Tiefen bis zu $25 \mathrm{~m}$ festgestellt, der See ist jedoch tiefer.

An dieser Lokalität wurde am 30. VIII. 1900 von BuFfa [1902] gesammelt; er meldet das Vorkommen von zwei Cladocerenspezies, nämlich:

Alona quadrangularis (O. F. MüLLER). Daphnia longispina O. F. MüLLER.

1) Vergl. hiezu unsere eigenen Beobachtungen vom Jahre 1922 im 1. Abschnitt dieser "Studien“ p. 407. 


\section{Hinterer Lahngangsee.}

Fu nd ortsnummer: 73. Z o ne: Nördliche Kalkalpen; Salzkammergut. Ge o g r a ph is ch e L a ge: Nordsteiermark, nordöstlich vom Grundlsee, im „Toten Gebirge“" gelegen. S e e h ö h e: ca. $1560 \mathrm{~m}$ ü. d. M. Gr ö B e: $0,07 \mathrm{~km}^{2}$ Oberfläche; $400 \mathrm{~m}$ lang, $200 \mathrm{~m}$ breit. F l u ßg e bi et: Traun-Donau. M a xi malti ef e: $19,91 \mathrm{~m}$.

Langhans [1911] meldet von diesem Fundort zwei Cladocerenspezies:

Acroperus harpae BAIRD. Alona costata G. O. SARS.

\section{Vorderer Lahngangsee.}

Fu nd ortsnu mmer: 74. Z o ne: Nördliche Kalkalpen; Salzkammergut. Ge $0 \mathrm{gr}$ a $\mathrm{ph}$ is $\mathrm{e} \mathrm{h}$ e L a ge: Nordsteiermark, nordöstlich vom Grundlsee, im "Toten Gebirge“ gelegen. S e e höh e: $1555 \mathrm{~m}$ ü. d. M. Flu Bg ebiet: Traun-Donau; entbehrt eines sichtbaren Abflusses. G r ö ß e: $0,194 \mathrm{~km}^{2}$ Oberfläche; $800 \mathrm{~m}$ lang, $400 \mathrm{~m}$ breit. M a x i malt i e f e: $77 \mathrm{~m}$; mittlere Tiefe $32,6 \mathrm{~m}$. Übrige Daten unbekannt.

LaNGhaiss [1911] wies das Vorkommen folgender Cladocera nach: Alona affinis (LEYDIG). Bosmina coregoni-longispina LEYDIG. Bythotrephes longimanus LEYdig. Diaphanosoma brachyurum (LiÉvin). Polyphemus pediculus (Linvé).

\section{Lambrechtersee.}

(Bisher nicht verö ffentlicht.)

Fundortsnummer: 75. Z o n e: Zentralalpen; Norische Alpen. Ge $0 \mathrm{graph}$ is ch e L a ge: Steiermark, südlich von Murau. S e e höh e: ca. $1050 \mathrm{~m}$ ü. d. M. Flu Bg e bi et: PriewaldbachLaßnitzbach-Mur-Drau. Gr ö Be: Kleines Wasserbecken von etwa 200 m Durchmesser. G e o l o g i e d e r U m g e b u n g: Liegt im Quarzphyllit.

Aus den von Dr. K. Holdhaus am 1. VII. 1921 gesammelten Planktonnetzfängen wurden von uns folgende Spezies bestimmt:

C o p e p o d a: Cyclops fuscus Jurine. Cyclops serrulatus Fischer.

Cla d o c e ra: Alona quadrangularis (0. F. Müller). Chydorus sphaericus 0. F. Müller. Peracantha truncata (0. F. Müller). Pleuroxus uncinatus BAIRD. Sidor crystallina (O. F. MÜLlER). Simocephalus vetulus (O. F. MüLleR).

Archiv f. Hydrobiologie, Suppl.-Bd. III. 


\section{Vorderer Langbathsee.}

Fu nd ortsnum mer: 76. Z o ne: Nördliche Kalkalpen; Salzkammergut. Ge o gra ph is ch e Lage: Oberösterreich; zwischen Attersee und Traunsee am Nordfuße des Höllengebirges gelegen. S e e höhe: 675 m. FluBgebiet: Langbathbach-Traun-Donau. Größ ße: $0,28 \mathrm{~km}^{2} ; 1175 \mathrm{~m}$ lang, $375 \mathrm{~m}$ breit. Maximaltiefe: $33,8 \mathrm{~m}$, im Mittel $10 \mathrm{~m}$. Übrige Daten nicht bekannt.

Über das Crustaceenplankton dieses Sees geben Iмноғ [1885], welcher seine Fänge am 27. VIII. 1884 ausführte, ferner Iмноғ [1890] un 1 KeIssLER [1907], welcher dort am 8. VII. 1906 fischte. folgende Liste:

Co p e p o da: Cyclops sp. [Iмноғ 1885, Keissler 1907]. Diaptomus sp. [Імноғ 1885]. Diaptomus bacillifer Koelbel [Імноғ 1890 sub D. montanus].

Cl a d o c e ra: Bosmina sp. [Inhof, Keissler]. Bythotrephes longimanus Leydig [ІмноF]. Daphnia sp. [Keissler]. Daphnia longispina var. hyalina Leydig [IмноF 1885 sub D. hyalina]. Diaphanosoma brachyurum (LiÉviv) [Імноғ]. Leptodora kindtii (Fоске) [Імноғ]. Polyphemus pediculus (Linné) [IмноF].

\section{Oberer Lanischsee.}

(Bisher nicht veröffentlicht.)

F u n d o r t s n u m m er: 77. Z o n e: Zentralalpen; Hohe Tauern. G e $0 \mathrm{gr}$ a ph i s c h e L a g e: Nordkärnten, Bezirk Gmünd, im Gebiete der Hafnerspitze gelegen. S e eh öh e: ca. $2300 \mathrm{~m}$ ü. d. M. Flu B ge bi et: Pöllatalbach-Malta-Lieser-Drau. Größe: Kleiner Schmelzwassersee. G e o log i e d e r U m g e bu ng: Granit. - Ohne makroskopisch entwickelter Wasserflora.

In den von Herrn Dr. K. Holdhaus am 8. VII. 1921 gesammelten Proben fanden sich außer einigen wenigen Insektenlarven keine Cladoceren und nur eine einzige Copepodenspezies, nämlich:

Cyclops serrulatus FisCHER.

\section{Unterer Lanischsee.}

(Bisher nicht veröffentlicht.)

Fundortsnummer: 78. S e ehöhe: ca. $2200 \mathrm{~m}$ ü. d. M. Alle übrigen Daten wie oben unter Nr. 77.

Die von Herrn Dr. K. Holdhaus am 8. VII. 1921 gesammelten Netzfänge enthielten:

Co p e p o d a: Cyclops serrulatus Fischer. 
Cl a d o c e r a: Alona sp. ? 1). Chydorus sphaericus O. F. Müller. Daphnia sp. ? ${ }^{1}$.

\section{Lansersee.}

Fu nd ortsnum mer: 79. Z o ne: Zentralalpen; Tuxer Tonschiefergebirge. Ge o g 1 a $\mathrm{ph}$ i s $\mathrm{ch}$ e Lage: Tirol, südlich von Innsbruck, am Fuße der Lanserköpfe-Patscherkofel gelegen. S e e h ö h e: $851 \mathrm{~m}$ ü. d. M. F l u Bg e b i e t: Sill-Inn-Donau. Gr ö ß e: ca. $200 \mathrm{~m}$ im größten Durchmesser. Ge ologis che und hydrographisehe Daten: Liegt im Tonglimmerschiefer. Nach Dalda Torre [1913] ein Restbecken in den Unebenheiten des einstigen Gletscherbodens. Wassertemperatur im Sommer bis zu $29^{\circ} \mathrm{C}$ gemessen. Enthält Fisehe.

Brenм [1907] erwähnt folgende Planktonformen:

Co p e pod a: Cyclops leuckarti Claus.

Cl a d o c e r a: Ceriodaphnia sp. (iuvenis). Sida crystallina (O. F. MüLLER).

\section{Lansermoorsee.}

Fu nd ortsnummer: 80. Z o ne: Zentralalpen; Tuxer Tonschiefergebirge. Ge o g r a ph is ch e La ge: Tirol, südlich von Innsbruck, am Fuße der Lanserköpfe-Patscherkofel gelegen. S e eh öh e: ca. $800 \mathrm{~m}$ ü. d. M. Flu Bg e bi et: Inn-Donau. Gr öße: ca $100 \mathrm{~m}$ größter Durchmesser. Ge ol o g i s che und h ydrograph is che $\mathrm{D}$ a t e n: Liegt im Schiefergebiet. Wie schon der Name sagt, handelt es sich bei dieser Fundstelle um ein versumpftes, mooriges Wasserbecken, das den Namen See nur insoferne verdient, als es perennierend ist.

Nach Heller [1871] und Brehm [1907] kommen dort folgende Spezies vor:

C o p e p o da: Cyclops serrulatus Fischer [Brenm 1907]. Cyclops viridis JuRINe [Heller 1871 sub C. brevicornis und C. clausii].

Cl a d o e e r a: Alonella excisa (Fischer) [BRenm 1907]. Chydorus sphaericus (O. F. MÜlLER) [BREHM 1907]. Simocephalus vetulus (O. F. MÜLLER) [BRehM 1907].

1) Da in beiden Fällen nar je ein einziges, schlecht erhaltenes Exemplar vorliegt, so dürfte es sich vielleicht um einen Netzrückstand handeln, der nicht von dieser Fundstelle stammt, weshalb wir das Vorkommen mit Fragezeichen versehen. Von Cyclops und Chydorus sind dagegen zahlreiche and gut erhaltene Individuen vorhanden. 


\section{Lasessee (Lago di Lases).}

F u nd ortsnu mmer: 81, Z o ne: Südliche Kalkalpen; Südtiroler Dolomiten. Ge o gr a ph i s e h e L a ge: Südtirol, nordöstlich von Trient, zwischen dem Val di Cembra und Val delle Pine. S e e höh e: 639 m ü. d. M. Flu Bgebiet: Avisio-Etsch. Grö Be: $700 \mathrm{~m}$ lang, $225 \mathrm{~m}$ breit; $0,16 \mathrm{~km}^{2}$ Oberfläche. M a xi malt i e f e: $31 \mathrm{~m}$ (nach Battista's Angaben nur 19,4 m). Ge olog i s e he und hydrographische Daten: In der Umgebung vorherrschend Phyllit, Verrucano und Porphyr. Nach Dalda Torre [1913] ist der See zur Überschwemmungszeit stark angeschwollen. Friert Mitte Dezember, taut Mitte März wieder auf. Buffa [1902] maß am 12. VIII. 1901 folgende Wassertemperaturen: Oberfläche $23^{\circ} \mathrm{C}$, in $20 \mathrm{~m}$ Tiefe $6,1^{\circ} \mathrm{C}$, in $31 \mathrm{~m}$ Tiefe $5,9^{\circ} \mathrm{C}$.

Buffa [1902] fand folgende Spezies:

C o p e p o d a: Canthocamptus minutus Claus.

$\mathrm{Cl}$ a d o c e r a: Bosmina longirostris (0. F. Müller). Diaphanosoma brachyurum (Liévin) [Buffa sub Daphnella br.]. Leptodora kindtii (Focke) [Buffa sub L. hyalina].

\section{Lauterersee.}

Fundorts nummer: 82. Z o ne: Zentralalpen; Stubaiergruppe der Ötztaleralpen. Ge ographische Lage: Tirol, am Übergang vom Gschnitztal in das Stubaital gelegen. S e ehö h e: ca. 2400 m. F l u B g e b i e t: Gschnitzbach-Sill—Inn-Donau. Gr ö ße: Kleines Hochgebirgsbecken von geringem Ausmaße (? Schmelzwassersee). Maximaltiefe: 4-8 m. Geologische und hydrographisehe Daten: Nach Brehm und Zederbauer [1904] erst im September eisfrei; dieselben Autoren maßen am 10. VIII. 1901 eine Oberflächenwassertemperatur von $5^{0} \mathrm{C}$, in $3 \mathrm{~m}$ Tiefe $4^{0} \mathrm{C}$.

Von Brehm und Zederbauer [1904] wird eine einzige Planktonform gemeldet:

Bosmina longirostris (O. F. MüLleR).

\section{Lavaronesee (Lago di Lavarone).}

Fu ndortsnu m mer: 83. Z o n e: Südliche Kalkalpen; Etschbuchtgebirge. Ge ographisehe Lage: Südtirol, südlich von Caldonazzo, nahe der italienischen Grenze. Flu Bge bi e t: AsticoBrenta (Adria). S e e h öh e: $1100 \mathrm{~m}$ ü. d. M. Gr ö B e: $350 \mathrm{~m}$ lang, $225 \mathrm{~m}$ breit; $0,054 \mathrm{~km}^{2}$ Oberfläche. Maximaltiefe: $15,8 \mathrm{~m}$. 
Ge o l o g i e d er U mge bu ng: Lias- und Dachsteinkalke. - Enthält F i s e h e: Barbe, Karpfen, Pfrille, Rotfeder.

Der See wurde am 1. VIII. 1903, 31. VII. 1904 und 29. VII. 1906 von LaRgaiolli [1906] untersucht und das Vorkommen folgender Spezies nachgewiesen:

Co p e p o da: Cyclops fuscus Jurine (iuvenis). Cyclops strenuus Fischer.

Cl a d o e e ra: Bosmina longirostris-cornuta Jurine. Diaphanosoma brachyurum (Líviv). Diaphanosoma brachyurum var. tridentinum LARG. Leptodora kindtii (FocKE).

\section{Ledrosee (Lago di Ledro).}

Fund orts nu m mer: 84. Z o n e: Südliche Kalkalpen. Etschbuchtgebirge. Ge ographische Lage: Südtirol, westlich vom Nordende des Gardasees. S e e h ö h e: $668 \mathrm{~m}$ ü. d. M. F l u Bg e bi e t: Ponalebach-Sarea-Mincio-Po (Adria). Gr ö ß e: 200 ha Oberfläche; 2,8 km lang, $1,4 \mathrm{~km}$ breit. Maximaltiefe: nach den Angaben von Pavesi [1884] nur $6 \mathrm{~m}$ tief, nach Dalla Torre [1913] 47,6 $\mathrm{m}$ tief! Geologische und hydrographische Daten: Liegt im geschichteten Moränenschutt und in Konglomeraten; Stausee. Friert im Winter zu. F is c h b e st a nd: Barbe, Forelle, Hecht, Koppe, Pfrille, Rotfeder, Schleie, Weißfisch u. a.

Pavesi [1884], welcher am 16. X. 1880 diesen Fundort untersuchte, gibt zwei Spezies an; außerdem wird er später von BREHM und ZeDERBAUER [1906] erwähnt.

Co pepoda: Cyclops viridis Jurine (sub C. brevicornis). Diaptomus steueri BreHM et ZederbaUer [1906].

Cla d o cera: Bosmina coregoni-longispina Leydig (sub B. longispina).

\section{Leonhardsee (Sankt-Leonhardsee).}

Fu n d ortsnum mer: 85. Z 0 n e: Zentralalpen; Norische Alpen. Ge ographis che Lage: Kärnten; nördlich von Villach. S e ehöh e: ca. $530 \mathrm{~m}$ ü. d. M. Flu Bge bi et: Afritz-Drau. Grö B e: Sehr kleines, teichartiges Wasserbecken; nähere Daten nicht ermittelt.

Über die Entomostrakenfauna hat schon Iмног [1890] berichtet; seine Angaben wurden später von Steuer [1897] übernommen; demnach kommen in diesem See folgende Genera und Spezies vor:

Copepoda: Cyclops sp. Diaptomus sp. 
C l a d o c e r a: Acroperus harpae Baird [Iмноғ sub A. leucocephalus]. Alona rectangula Sars [Imно sub A. lineata]. Bosmina sp. Daphnia sp. Diaphanosoma brachyurum (LiÉvin). Iliocryptus sordidus (LiÉvin). Peracantha truncata (O. F. MülLeR) [ImhoF sub Pleuroxus tr.].

\section{Leopoldsteinersee ${ }^{1}$ ).}

(Bisher nicht veröffentlicht.)

F u n d o r t s n u m mer: 86. Z o n e: Nördliche Kalkalpen. Österreichische Kalkalpen; Hochschwabgebiet. G e $0 \mathrm{gr}$ a $\mathrm{ph}$ is c h e $\mathrm{L}$ a g e: Nordoststeiermark, zwischen Eisenerz und Hieflau gelegen. S e e h ö h e: 619 m ü. d. M. Flu Bg e b i et: Erzbach-Enns-Donau. Gr ö B e: Ungefähr $1400 \mathrm{~m}$ lang, $500 \mathrm{~m}$ breit. M a xi m a $1 \mathrm{t}$ i e $\mathrm{e}$ e: $32 \mathrm{~m}$. Von KeIssLer wurde Ende Juli 1911 an der Oberfläche eine Wassertemperatur von $17^{\circ} \mathrm{C}$ gemessen (Maximaltemperatur!).

Aus den von Keissler in den Jahren 1910 und 1911 gesammelten Planktonproben wurden von uns folgende Spezies determiniert:

Co p e poda: Cyclops strenuus Fischer. Diaptomus gracilis G. O. SARS.

Cla d o cera: Bosmina coregoni BAIRD. Daphnia longispina O. F. MÜLLeR.

\section{Levicosee.}

F u n d o r t s n u m mer: 87. Z o n e: Südliche Kalkalpen; Grenzlinie zwischen Etschbuchtgebirge und Südtiroler Dolomiten. Ge ogra ph is ch e L a ge: Südtirol, bei Levico nächst dem Caldonazzosee. S e e höh e: $449 \mathrm{~m}$ ü. d. M. Flu Bge bi et: Brenta (Adria). G r ö B e: $2,48 \mathrm{~km}$ lang, $680 \mathrm{~m}$ breit; $1,06 \mathrm{~km}^{2}$ Oberfläche. M a x i m a l tiefe: $35 \mathrm{~m}$. Geologische und hydrographische Dat e $\mathrm{n}$ : Stausee, einstmals mit dem Caldonazzosee eine gemeinsame Wasseransammlung bildend. Enthält zahlreiche Fische: Aal, Barbe, Forelle, Hecht, Karpfen, Koppen, Neunauge, Rotfeder, Schleie u. a.

Pavesi [1884] zählt für diesen Fundort folgende Spezies auf:

C o p e p o d a: Cyclops serrulatus Fischer.

$\mathrm{Cl}$ a d o c e ra: Alona quadrangularis (O. F. MülLer). Bosmina longirostris (O. F. MÜLLER). Diaphanosoma brachyurum (LiÉvin). Leptodora kindtii (FOCKE).

1) Über diesen Fundort liegt eine Publikation von K. v. KeIssLer vor („Untersuchungen über die Periodizität des Phytoplanktons des Leopoldsteinersees in Steiermark". Archiv f. Hydrobiol., vol. 6, 1910, p. 480), in welcher bereits das Vorkommen der beiden Genera Cyclops und Diaptomus erwähnt wird. 


\section{Lichtsee.}

F u n d o r t s n u m mer: 88. Z o n e: Zentralalpen; Stubaiergruppe der Ötztaleralpen. Ge $0 \mathrm{gr}$ a phis che $\mathrm{L}$ a ge: Tirol. westlich von Gries am Brenner, am Trunnerjoch (Übergang vom Obernbergertal in das Gschnitztal) gelegen. S e e h ö h e: $2100 \mathrm{~m}$ ü. d. M. ${ }^{1}$ ) F l u B g e bi e t: Obernbergerseebach - Sill-Inn-Donau. Gr ö B e: $200 \mathrm{~m}$ lang, $150 \mathrm{~m}$ breit. M a ximaltiefe: Unbekannt, rermutlich wenige Meter. Geologische und hydrographisehe Daten: Das Seebecken liegt in Carbonschichten. Ufer wenig steil; bis zum Sommer vereist. Oberflächliche Was sertemperaturam 4. IX. 1902 betrug $9^{\circ} \mathrm{C}$.

Brehm und Zederbauer [1904] fanden folgende Planktonarten:

Co p e poda : Cyclops sp. (iuvenis). Diaptomus denticomis WierZEJSKI.

\section{Loppiosee (Lago di Loppio).}

Fundortsnummer: 89. Z o n e: Südliche Kalkalpen; Etschbuchtgebirge. Ge $0 \mathrm{~g} r$ a $\mathrm{ph}$ i s e h e L a g e: Südtirol, östlich rom Nordende des Gardasees, Bezirk Mori, gelegen. S e e h ö h e: $220 \mathrm{~m}$ ï. d. M. F l u ß g e bi e t: Camerasbach-Etsch (Adria). Gr ö ß e: $0,6 \mathrm{~km}^{2}$ Oberfläche; $1830 \mathrm{~m}$ lang, $400 \mathrm{~m}$ breit. M a xi malt i e f e: $4,1 \mathrm{~m}$. Ge 0 logische und hydrographische Daten: Liegt im Liaskalk; Einsturzkesselsee. Von Brehm und Zederbader wurde am 27. XII. 1901 eine oberflächliche Wassertemperatur von $3^{0} \mathrm{C}$, in $2 \mathrm{~m}$ Tiefe von $4^{0} \mathrm{C}$ gemessen (gleichzeitige Lufttemperatur $4^{\circ} \mathrm{C}$ ). Im Winter quantitativ und qualitativ planktonarm [nach Brenm und Zederbauer 1904]. Enthält: Aal, Barbe, Hecht, Karpfen, Rotfeder, Schleie, Weißfiseh u. a.

Dieses Wasserbecken wurde ron Pavesi [1884], Brehm und ZederBaUer [1904] und Largatolli [1910] untersucht; demnach kommen folgende Formen vor:

Co p e poda: Cyclops sp. [Pavesi]. Cyclops serrulatus Fischer [LARGAIOLLI].

Cla d o cera: Acroperus angustatus G. O. Sars [Largaioldr]. Alona rectangula G. O. Sars [Largaiolli sub Lynceus r.]. Bosmina.

1) Nach einer neuen Aufnahme auf der Karte des Brennergebietes, herausgegeben vom Deutsch-Österr. Alpenverein im Jahre 1920. BREHM und Zederbauer [1904], sowie Dalla Torre [1913] vermerken die Seehöhe mit ca. $2200 \mathrm{~m}$. 
Tongirostris-comuta JuRINe [PAVESI, LaRgarolli]. Ceriodaphnia pulchella G. O. Sars [Largaiolli]. Ceriodaphnia quadrangula (O. F. Müller) [Pavesi]. Diaphanosoma brachyurum (Liéviv) [Largaioldi]. Diaphanosoma brachyurum var. tridentinum LARGAIOLLI. Pleuroxus trigonellus (O. F. Müller) [Pavesi; Largaiolli]. Sida crystallina (O. F. Müller) [Largatoliti].

In dem Berichte von Zederbauer und Brenm [1904], welche ihre Planktonfänge am 27. XII. 1901 ausführten, wird besonders die Zooplanktonarmut betont und nur das Vorkommen einiger Nauplien angegeben; es wäre dies ein Bild von den winterlichen Verhältnissen. Pavesi [1884] führte seine Fänge am 16. X. 1880 aus, Largaiolli [1910] durch mehrere Jahre während des Monates August.

\section{Lunzer Mittersee.}

F u n d o r ts n u m m er: 90. Z o n e: Nördliche Kalkalpen; Österreichische Kalkalpen. G e $0 \mathrm{gr}$ a $\mathrm{ph}$ i s e h e $\mathrm{L}$ a g e: Niederösterreich, bei Lunz. S e e höh e: $763 \mathrm{~m}$ ü. d. M. Fl u Bg e bi et: Seebach-YbbsDonau. Gr ö ße: 2,5 ha Oberfläche; $320 \mathrm{~m}$ lang, $150 \mathrm{~m}$ breit. M a ximaltiefe: $2,5-3 \mathrm{~m}$. Geologische und hydrographisehe D at e n: Grundwassersee mit starker Strömung. Nach HaLbFass [1913] sind alle drei Lunzerseen postglazial entstanden und verdanken ihre Existenz der diluvialen Vergletscherung des Dürrensteins (1877 m), von dem eine Gletscherzunge in das Seebachtal reichte und hier eine Übertiefung gebildet hat. - Wassertemperatur im Sommer: $6,5^{0} \mathrm{C}$.

Die folgende Entomostrakenliste gründet sich auf die Mitteilungen von Woltereck [1906] und Brehm [1907, 1909 und 1913/14].

C o p e p o da: Canthocamptus staphylinus (Jurine) [Brehm 1907 und 1909]. Cyclops fimbriatus Fischer [Brehm 1907, 1909]. Cyclops serrulatus Fischer [Woltereck 1906, BRehm 1907, 1909]. Cyclops viridis Jurine [Woltereck 1906, Brehm 1907, 1909, 1913/14].

Clad o cera: Acroperus harpae (BAIRD) [Woltereck 1906, BREHM 1907 und 1909]. Alona affinis (LeYdig) [Woltereck 1906, BReHM 1907 und 1909 sub Lynceus aff.]. Bosmina coregoni BaIRd [BReHM 1909]. Chydorus sphaericus (O. F. MüLler) [Woltereck 1906, Brehm 1907 und 1909]. Daphnia sp. (iuvenis) [BREHм 1909]. Daphnia longispina O. F. Müller [Woltereck 1906, BrehM 1907]. Iliocryptus sordidus (LiÉviN) [BREHM 1909 vnd 1913/14]. 


\section{Lunzer Obersee.}

Fundortsnummer: 91. Z o ne, ge ographische Lage, FluBgebiet und geologische Daten wie bei Nr. 90 (L unzer Mittersee). - S e ehöh e: 1113 m ü. d. M. Größe: 18 ha Oberfläche; $700 \mathrm{~m}$ lang, $400 \mathrm{~m}$ breit. Il a ximaltiefe: $15 \mathrm{~m}$.

Woltereck [1906] und BREhm [1907, 1913 und 1913/14] geben für diesen See folgende Spezies an:

Co pepoda: Canthocamptus rhaeticus SchMeil [Brehm 1913]. Canthocamptus staphylinus (JURIne) [Woltereck 1906, Brehm 1907]. Canthocamptus zschokkii Schmeil [BREhM 1913]. Cyclops albidus Jurine [Woltereck 1906, Brehm 1907]. Cyclops fuscus Jurine [Woltereck 1906, Brehm 1907]. Cyclops serrulatus Fischer [Woltereck 1906, Brenm 1907]. Cyclops strenuus Fischer [Woltereck 1906]. Diaptomus denticornis Wierzejski [Woltereck 1906, Brehm 1907, 1913/14]. Moraria schmeili van Douwe [BRenm 1913].

C l a d o c e r a: Acroperus harpae Baird [Woltereck 1906, Brehur 1907]. Alona guttata G. O. Sars [Woltereck 1906, Brehm 1907 sub Lynceus guttatus]. Alonella nana (BAIRD) [WoLtereck 1906, BREHM 1907]. Bosmina coregoni Baird [Woltereck 1906, Brehm 1907]. Chydorus sphaericus (O. F. MÜller) [Woltereck 1906, Brehm 1907], Daphnia longispina O. F. Müller [Woltereck 1906, Brehm 1907]. Peracantha truncata (O. F. Müller) [Woltereck 1906, Brehm 1907]. Pleuroxus trigonellus (O. F. MülLer) [Woltereck 1906, Brehm 1907]. Polyphemus pediculus (Linvé) [Woltereck 1906, Brenu 1907, 1913/14]. Scapholeberis mucronata (O. F. Müller) [Woltereck 1906]. Simocephalus vetulus (O. F. MÜlLer) [Woltereck 1906, Brehy 1907].

\section{Lunzer Untersee.}

Fundortsnum mer: 92. Z o ne, ge ograph is che Lage, FluBgebiet und geologische Daten wie bei Nr. 90 (Lunzer Mittersee). - S e e h öh e: $608 \mathrm{~m}$ ü. d. M. Gr ö ße: 68 ha Oberfläche; $1,7 \mathrm{~km}$ lang, $600 \mathrm{~m}$ breit. M a x i malt i e f e: 35 (34) m; mittlere Tiefe $20 \mathrm{~m}$. Wassertemperat ure n nach Messungen von Brehy und Zederbauer [1906], gemessen am 27. V.: Oberfläche $13,5^{\circ} \mathrm{C}$, in $5 \mathrm{~m}$ Tiefe $11^{\circ} \mathrm{C}$, in $10 \mathrm{~m}$ Tiefe $8^{\circ} \mathrm{C}$.

Die folgende Speziesliste gründet sich auf die Angaben von KeIsSLeR [1900], Steuer [1901], Brehy und Zederbauer [1906], Woltereck [1906], Вrehм [1907, 1913 und 1913/14]. 
Co pepoda: Canthocamptus echinatus var. luenensis SchmeIL [Brenm 1913, 1913/14]. Canthocamptus rhaeticus Schmeil [Brehm und Zederbauer 1906, Brehm 1913/14]. Canthocamptus schmeili Mrazek [Breni 1913/14]. Canthocamptus staphylinus (Jurine) [Woltereck 1906, Вкенм 1913 sub staph. var.]. Canthocamptus vejdovskyi MrazeK [13renm 1913]. Canthocamptus wierzejskii Мrazek [Вrenm 1913, 1913/14]. Canthocamptus zschokkei SchmeIl [Brenm 1913, 1913/14]. Cyclops affinis Sars [Woltereck 1906]. Cyclops albidus Jurine [Вrehm 1907]. Cyclops bicolor Sars [Woltereck 1906]. Cyclops fuscus Jurine [Woltereck 1906. Brehm 1907]. Cyclops serrulatus Fischer [Woltereck 1906. Brehm 1907]. Cyclops strenuus Fischer [Keissler 1900, Brehm und Zederbauer 1906, Woltereck 1906, Brehm 1907]. Diaptomus gracilis G. O. Sars [Keissler 1900, Brehu und Zederbauer 1906, Woltereck 1906, Brehu 1907]. Moraria schmeili van Douwe [Brehm 1913, 1913/14].

Cl a d o c e r a: Acroperus angustatus G. O. Sars [Brenm 1913/14]. Acroperus harpae Baird [Woltereck 1906, Brehm 1907]. Alona costata G. O. Sars [Woltereck 1906 sub Lynceus costatus]. Alona guttata G. O. Sars [Woltereck 1906 sub Lynceus guttatus]. Alonella nana (BAtrd) [Woltereck 1906, BRehm 1907]. Bosmina coregoni-longispina Leydig [Keissler 1900 sub B. bohemica, Steuer 1901, Brehm und Zederbauer 1906, Woltereck 1906, Вrehm 1907 sub B. coregoni]. Ceriodaphnia laticaudata P. E. Müller [ВREнm 1913/14]. Chydorus sphaericus (O. F. Müller) [Woltereck 1906, Brehm 1907]. Daphnia longispina var. hyalina Leydig [KeIssler 1900, Brehm und Zederbauer 1906 sub D. hyalina, Woltereck 1906, Brehm 1907 sub D. longispina]. Graptoleberis testudinaria (FISCHER) [WOLTERECK 1906, BREHM 1907]. Peracantha truncata (O. F. Müller) [Woltereck 1906]. Simocephalus vetulus (O. F. Mülder) [Woltereck 1906, Brehm 1907¹)].

\section{Lünersee.}

F u n d or t s n u m m e r: 93. Z o n e: Nördliche Kalkalpen; Rhätikongruppe der Allgäuer Alpen. Ge o graph is che Lage: Vorarlberg, nächst der Schweizer Grenze am Fuße der Scesaplana. S e e h ö h e: 1943 m ü. d. M. Flu Bg e biet: Alvier-Ml-Rhein. Größ e:

1) Eine zusammenfassende Übersicht über die Copepoden- und Cladocerenfauna aller 3 Seen des Lunzer Gebietes steht noch aus; ihre Veröffentlichung soll von V. ВREHM für die nächste Zeit geplant sein. Über Hydrophysik und Chemiesmus der Lunzer Seen liegen ausführliche Arbeiten von GöтzIngER [Internat. Revue f. Hydrogr. 1908, 1912,1917 ] und von Muldey und Wittmann [ebenda 1914] vor. 
ca. $1 \mathrm{~km}^{2}$ Oberfläche; Umfang $6 \mathrm{~km}$. II a ximaltiefe: $102 \mathrm{~m}$. Geologische und hydrographische Daten: Ein Gletschererosionsbecken. Liegt in Kalkgesteinen (Dolomit und Kreidekalk). Am Seeufer anstehend Raiblerschichten mit Alabaster. Von Mitte Oktober bis Juni vereist. Oberflä che $n$ was sertemperat ur nach ZschokкE's Messungen rom August 1900: 10-11.700 C. - En thält $\mathrm{Fi}$ is ch e (Koppe).

Der See wurde eingehend ron ZschokKe [1890 u. 1900] untersucht und die Determination der Copepoden von Schmerl [1893] revidiert; demnach kommen folgende Spezies vor:

Copepoda: Canthocamptus echinatus var. luenensis ScHmeIL [ZschoKke 1900]. Canthocamptus minutus Claus [ZschokKe 1900]. Canthocamptus rhaeticus SchueIL [ZschokKE 1900]. Canthocamptus schmeili var. hamata ScHмEIL [ZschокKE 1900]. Cyclops fimbriatus Fischer [Zschokke 1900]. Cyclops serrulatus Fischer [ZschoKke 1900]. Cyclops strenuus Fischer. Cyclops vernalis Fischer. Diaptomus bacillifer KOELBEL.

Cla d o e er a: Chydorus sphaericus O. F. Müller. Daphnia longispina O. F. MülLer. Daphnia pulex (DE GEeR). Rhynchotalona rostrata (КосH) [ZscноККE sub Lynceus rostratus].

\section{Großer Magdalenensee.}

F u n d o r t s n u m m e r: 94. Z o n e: Südliche Kalkalpen; Klagenfurter Becken. Ge o graph is che L g e: Kärnten, östlich von Villach. S e e höh e: $491 \mathrm{~m}$ ü. d. M. Flu Bg e b i e t: Seebach-Drau. Gr ö B e: ca. $525 \mathrm{~m}$ lang, $300 \mathrm{~m}$ breit. T i e f e: Unbekannt, ebenso die übrigen Daten.

Über die Crustaceenfauna dieses Wasserbeckens haben Iмноғ [1890] und STEUER [1897] ausführliche Angaben gemacht; demnach lautet die Artenliste:

Co p e p o d a: Canthocamptus staphylinus (JuRINe) [STEuer 1897]. Cyclops sp. [Iмноғ 1890]. Cyclops albidus Jurine [Steuer 1897]. Cyclops fuscus Jurine [Steuer 1897]. Cyclops macrurus G. O. Sars [Stecer 1897]. Cyclops serrulatus Fischer [STEuer 1897]. Cyclops strenuus Fischer (Steuer 1897]. Diaptomus gracilis G. O. Sars [Steuer 1897].

Cla do cer a: Acroperus harpae BAIRd [IMHof 1890 und Steuer 1897 sub A. leucocephalus]. Alona costata G. O. Sars [ImhoF 1890]. Alona guttata G. O. Sars [Steuer 1897]. Alona quadrangularis (O. F. MüLLER) [Steuer 1897]. Bosmina sp. [Імноғ 1890]. Bosmina coregoni- 
longicomis Schoedler [STeuer 1897 sub B. longicornis]. Camptocercus rectirostris (Schoedler) [Steuer 1897]. Ceriodaphnia sp. [Iмноғ 1890]. Chydorus latus Sars [Steuer 1897]. Diaphanosoma brachyurum (LiÉvin) [Тмноғ 1890]. Eurycercus lamellatus (O. F. Müller) [Steuer 1897]. Macrothrix rosea (Jurine) [Imнof 1890]. Monospilus dispar G. O. SARS. [Iмног 1890 sub M. tenuirostris]. Peracantha truncata (O. F. MüLler) [Імноғ 1890 und Steuer 1897 sub Pleuroxus tr.]. Pleuroxus trigonellus (O. F. Müller) [STeuer 1897]. Sida crystallina (O. F. Müller) [Steuer 1897]. Simocephalus exspinosus (КосH) [STEUER 1897].

\section{Kleiner Magdalenensee.}

Fundortsnummer: 95. Zone, ge ographische Lage, Flußgebiet wie bei Fundortsnummer 94. Größ e: ca. $140 \mathrm{~m}$ im Durchmesser. Tieie und übrige Daten nicht ermittelt.

Auch für diesen See bilden die Mitteilungen Iмноғ's [1890] die einzige Originalgrundlage, während StEuER [1897] lediglich diese Angaben in seiner Zusammenstellung übernommen hat. Es wurden nachgewiesen:

Co p e p od a: Cyclops sp.

Cladocera: Bosmina sp. Ceriodaphnia sp. Diaphanosoma brachyurum (LIÉviN). Scapholeberis mucronata (O. F. MüLLER).

Mangartsee siehe Unterer Weißenfelsersee (Nr: 185).

\section{Mariasteinersee.}

F u n d o r t s n u m mer: 96. Z o n e: Nördliche Kalkalpen. Nordtiroler Kalkalpenzug. Ge o gra ph is e h e Lage: Nordtirol, Unterinntal nördlich von Wörgl. Seehöhe: $534 \mathrm{~m}$ ü. d. M. Fluß ge biet: Nasenbach-Inn-Donau. Grö Be: Sehr kleines Wasserbecken von geringer Tiefe; verschilfter Seichtsee. Ge ol ogi is ch e und hydrographische Daten: Nach Müllner [1905] ein Stausee, der in Kalkschottern auf Dolomitkalkgrundlage eingebettet liegt; nach Dalla ToRre [1913] durch einen Bergsturz entstanden. F i s e h be st and: Aitel, Hecht, Pfrille, Rotfeder.

Brenm [1907] konnte folgende Formen nachweisen:

$\mathrm{C}$ o p e p o d a: Cyclops sp. (iuvenis).

Cla d o c era: Acroperus harpae BAIRd. Alonella nana (BAIRD). Eurycercus lamellatus (O. F. Müller). Peracantha truncata (O. F. Müller). 
Mattsee siehe Niedertrumersee (Nr. 109).

\section{Millstättersee.}

Fundortsnummer: 97. Z one: Zentralalpen; Norische Alpen. G e $0 \mathrm{gr}$ a ph is e h e L a g e: Kärnten, Millstatt_Spital. S e e h öh e: 580 m ü. d. M. Flu Bg e bi e t: Riegerbach-Lieser-Drau. Gr ö ß e: 13,25 km² Oberfläche; gestrecktes Becken von $11 \mathrm{~km}$ Länge und durehschnittlich $1 \mathrm{~km}$ Breite. Maximaltief e: $140,7 \mathrm{~m}$; im Durchschnitt $86,45 \mathrm{~m}$ tief. Ge ol o g i s ch e und h ydrograph i sche Daten: Liegt in Schiefergestein. Nach HalbFass [1913] ist der Millstättersee ein Differenzbecken, von den Schuttkegeln des Liesertales über den eigentlichen Rand emporgestaut. Eingehende Wassertemperaturmessungen stammen von RicHTER [1897]; demnach sind in den Sommermonaten Juli und August $\mathrm{Ob}$ erflä ch entempe $\mathrm{raturen}$ von durchschnittlich $20^{\circ} \mathrm{C}$ um die ersten Nachmittagsstunden häufig, oft auch höhere, so daß sie unter besonderen Witterungsverhältnissen über $28^{\circ} \mathrm{C}$ erreichen können. - Nach HaEMPEL [i. l.] enthält der See folgende F is c h e: Alburnus lucidus, Esox lucius, Gobio fluviatilis, Lota vulgaris, Perca fluviatilis, Rhodeus amarus, Scardinius erythrophthalmus, Silurus glanis, Squalius cephalus, Trutta fario und T. lacustris.

Über die pelagische Crustaceenfauna dieses Sees haben Iмноғ [1890]. Steuer [1897 ex Imhof!], Keissler [1904] und Brehm und ZederBAUER [1905] berichtet; durch O. HaEmpel [i. l.], welcher uns seine Untersuchungsergebnisse freundlichst zur Verfügung stellte, hat sich die Liste der Genera und Spezies noch wesentlich vergrößert, so daß nun das Vorkommen folgender Formen gemeldet werden kann:

Copepoda: Canthocamptus sp. [Haempel i. l.]. Cyclops sp. [Imhof, Steuer, Brehm und Zederbader]. Cyclops fimbriatus Fischer [Haempel i. 1.]. Cyclops serrulatus Fischer [Haempel i. l.]. Cyclops strenuus Fischer [Haempel i. 1.]. Cyclops viridis Jurine [Haempex i. 1.]. Diaptomus sp. [Imhof, Steuer, Keissler]. Diaptomus castor Jurine [ImhoF, Steuer, Haempel i. 1.]. Diaptomus gracilis Sars [HaemPEL i. 1.].

$\mathrm{Clad}$ o cera: Acroperus harpae Baird [Haempel i. 1.]. Alona quadrangularis (0. F. MüLleR) [HAEMPEL i. 1.]. Alonella sp. [HaEMPEL i. 1.]. Bythotrephes longimanus Leydig [Imнof, Steuer, Keissler]. Ceriodaphnia pulchella SARD [HAEMPEL i. 1.]. Chydorus sphaericus O. F. MülleR [Haempel i. 1.]. Daphnia sp. [Keissler]. Daphnia longispina var. 
mecullata Sars [Brehm und Zederbauer]. Daphnia longispina var. Tyalina Leydig forma pellucida P. E. Müller [Haempel i. 1.]. Diaphanosoma brachyurum (Lí́vin) [Imhof, Steuer, Brehy und Zederbauer, Haempel i. I.]. Eurycercus lamellatus (O. F. Müller) [Haempel i. 1.]. Leptodora kindtii (Focke) [ImhoF, Steuer, Keissler, Brehm und Zederbauer, Haempel i. l.]. Peracantha truncata (O. F. Müller) [Haempel i. I.]. Sida crystallina (O. F. Müller) [Imhof, Steuer, HaemPEL i. 1.]. Simocephalus vetulus (O. F. MÜller) [Haempel i. 1.].

\section{Misurinasee.}

Fu nd ortsnummer: 98. Z o ne: Südliche Kalkalpen; Südtiroler Dolomiten. Ge ographis che Lage: Italien, nächst der Grenze von Südtirol, am Fuße des Monte Cristallo. S e e h ö h e: $1755 \mathrm{~m}$ ü. d. M. Flu B g e bi et: Ansiei-Piave (Adria). Gr ö B e: ca. $700 \mathrm{~m}$ lang und $200 \mathrm{~m}$ breit. M a x i m a l t i e f e: Unbekannt. G e $0 \mathrm{l} 0 \mathrm{~g}$ i s e h e und hydrographisehe Daten: Liegt in Cassianermergel und Dolomitkalken. Oberflä chenwassertemperatur, von Brehm und Zederbauer [1905] am Abend des 3. VIII. 1903 gemessen, betrug $10^{\circ} \mathrm{C}$.

Von Brehm und Zederbauer [1905] werden zwei einzige Planktonformen gemeldet, nämlich:

C o p e p oda: Diaptomus denticornis WIERzEJSKI.

Cl a d o c e r a: Daphnia longispina var. hyalina Lẹdig f. microcephala SARS.

Mittersee am Fernpaß.

F u n d ortsn u m mer: 99. Z o n e: Nördliche Kalkalpen; Nordtiroler Kalkalpen. Ge ogra ph is ch e Lage: Nordtirol, Bezirk Biberwier, am Fernpaß gelegen. S e e höh e: $1086 \mathrm{~m}$ ü. d. M. (nach Dalla Torre [1913] $1134 \mathrm{~m}$ ü. d. M.). F l u Bg e bi e t: LoisachIsar-Donau. Gr ö ß e: Ungefähr $200 \mathrm{~m}$ im Durchschnittsdurchmesser. Maximaltiefe: Unbekannt. Ge ologische und hydrograph is che Daten: Der See gehört nach Böнm [1886] zu den echten Felsbecken, entstanden durch Einbruch. Enth ält: F or elle, S a i bling.

BREнм [1907] meldet folgende Spezies:

C o p e p o da: Cyclops serrulatus var. denticulata GraEter.

Cl a d o e e ra: Acroperus harpae BAIRD. Alona costata SARs (sub Lynceus cost.) Peracantha truncata (O. F. MüLler). 


\section{Mondsee.}

F u n d o r t s n u m mer: 100. Z o n e: Nördliche Kalkalpen; Salzkammergut. Ge ographisehe Lage: Oberösterreich, Mondsee östlich von Salzburg. S e ehöh e: $479 \mathrm{~m}$ ü. d. M. Flu Bg e bi et: Seeache-Ager-Traun-Donau. Größ e: 14,21 km² Oberfläche; ungefähr $10 \mathrm{~km}$ lang und durchschnittlich $1 \frac{1}{2} \mathrm{~km}$ breit. M a ximalti efe: $68,3 \mathrm{~m}$, im Nittel $36 \mathrm{~m}$. Ge $0 \mathrm{log}$ is ehe und hydrographische Daten: Nach HalbFass [1913] eine in festes Gistein eingesenkte Felswanne (Zungenbecken). Wassertemperaturmessungen stammen von Breni und Zederbauer [1906], wurden jedoch am 30. XII. 1901 und 24. III. 1902 ausgeführt; im Dezember betrug die Temperatur des Wassers an der Oberfläche bis in $5 \mathrm{~m}$ Tiefe $4^{\circ} \mathrm{C}$, im März $3^{0} \mathrm{C}$ in $0 \mathrm{~m}, 3,5$ in $2 \mathrm{~m}, 4^{0} \mathrm{C}$ in $5 \mathrm{~m}$ Tiefe. $-\mathrm{En} \mathrm{th}$ ä $\mathrm{lt}$ die Fische: Abramis brama, Alburnus lucidus, Anguilla vulgaris, Aspius rapax, Barbus fluviatilis, Coregonus fera, Cottus gobio, Esox lucius, Lota vulgaris, Perca fluviatilis, Phoxinus laevis, Salmo salvelinus, Scardinius erythrophthalmus, Squalius cephalus, Trutta lacustris.

Die folgende Liste gründet sich auf die Beobachtungen von ImHOF [1885], welcher am 24. VIII. 1884 sammelte, StEuER [1901], BRehy und Zederbauer [1906], welche, wie erwähnt, das Winterplankton untersuchten, und Langhans [1911]; demnach wurden nachstehende Formen nachgewiesen:

C o p e p o d a: Cyclops sp. [Імноғ 1885]. Cyclops strenuus Fischer [Brehm und Zederbauer 1906]. Diaptomus sp. [Iмhof 1885]. Diaptomus gracilis G. O. Sars [Brehm und ZederbaUer 1906]. Diaptomus laciniatus LILlJEBorg [STEUeR 1901].

C l a d o e e r a: Bosmina sp. [Inhof 1885]. Bosmina coregoni Baird [Brehy und Zederbauer 1906]. Bythotrephus longimanus Leydig [Імноғ 1885]. Daphnia longispina var. cucullata SARS [Імноғ 1885 sub D. Kahlbergensis Schoedler rnd Langhans 1911 sub Hyalodaphnia sarsi Langh.]. Daphnia longispina var. hyalina Leydig [ІмноF sub D. gracilis Hellich und Brehm und Zederbauer 1906, Langhans 1911 sub D. hyalina]. Diaphanosoma brachyurum (Liévin) [Імноғ 1885]. Leptodora kindtii (Focke) [IмноF 1885]. Sida crystallina (O. F. MüLleR) [LANGHANS 1911].

\section{Großer Montigglersee.}

F u n d o r t s n u m m e r: 101. Z o n e: Südliche Kalkalpen; Etsel buchtgebirge. Ge $0 \mathrm{gr}$ a ph i s ch e L a ge: Südtirol, im sogenannten „Überetsch“, östlich von Kaltern gelegen. S e e h ö h e: $500 \mathrm{~m}$ ü. d. M. 
Flu by e bie t: Etsch. Grö Be: $171600 \mathrm{~m}^{2}$ Oberfläche; $720 \mathrm{~m}$ lang, $290 \mathrm{~m}$ breit. Maximaltiefe: $12 \mathrm{~m}$. Geologische und h ydrograph ische Daten: Liegt in Porphyr. Nach Halbfass [1913] eine Flußkolke im Fels, bereits zur Eiszeit die jetzige Tiefe erreichend. Von Mitte Dezember bis März rereist. Huber [1906] stellte im Laufe eines Jahres 0 berflächenwassertemperaturen von $3,1-4,8^{\circ} \mathrm{C}$ fest. Was serfarbe: Braungrün. Fischbestand: Barsch, Brachsen, Hecht, Karpfen, Rotfeder, Schleie (Aal nach Dalla Torre [1913] einst vorhanden, jedoch abgewandert).

Die von Huber [1906] während eines Jahreszyklus durchgeführten Untersuchungen ergaben das Vorkommen folgender Arten:

Co pe p oda: Cyclops fuscus Jurine. Cyclops leuckarti Claus. Cyclops macrurus SARS. Cyclops oithonoides SARS. Cyclops serrulatus Fischer. Cyclops strenuus Fischer.

$\mathrm{Cl}$ a d o e e ra: Acroperus angustatus Sars. Alona affinis (LEYDIG). Alona guttata SARS. Alona quadrangularis (0. F. MüLLER). Alonella excisa (Fischer) [Huber sub Pleuroxus exc.]. Bosmina longirostriscornuta JuRINE. Ceriodaphnia pulchella SARS. Chydorus sphaericus (O. F. Müller). Daphnia longispina var. hyalina Leydig [Huber sub D. hyalina]. Diaphanosoma brachyurum (LiÉvin) [HuBER sub Daphnella br.], Graptoleberis testudinaria (FISCHER) [HubER sub Alona t.]. Leptodora kindtii (Focke). Peracantha truncata (O. F. MüLleR) [Huber sub Pleuroxus tr.]. Pseudalona latissima (KuRz) [Huber süb Alonopsis l.].

\section{Kleiner Montigglersee.}

Fundortsṇummer: 102. Seehöhe: $510 \mathrm{~m}$ ü. d. M. Gr ö B e: $49490 \mathrm{~m}^{2}$ Oberfläche; $300 \mathrm{~m}$ lang, $220 \mathrm{~m}$ breit. M a xi ma ltiefe: $14 \mathrm{~m}$. - Z Z ne, geographisehe Lage, Flußgebiet, Geologische Daten siehe wiebeim groben Montigglersee (Nr. 101) angegeben.

Bei diesem See erstrecken sich die Angaben Huber's [1906], welcher hier seine Beobachtungen in der Zeit vom 9. X.-15. XII. 1902 anstellte, nur auf die p e l a g i s c h e Crustaceenfauna, von welcher folgende Spezies genannt werden:

Co p e p o da: Cyclops strenuus (Fischer).

C l a d o c e r a: Bosmina longirostris-cornuta JuRINe. Ceriodaphnia pulchella SARS. Daphnia longispina var. hyalina LEydig. Diaphanosoma brachyurum (LIÉVIN). 


\section{Oberer Mühldorfersee.}

F u n d o r t s n u m m e r: 103. Z o n e: Zentralalpen; Hohe Tauern. Ge $0 \mathrm{gr}$ a $\mathrm{ph}$ is ch e L a ge: Kärnten, nördlich von Sachsenburg bei Mühldorf im Mölltale, im Gebiete der Hohen Leier gelegen. S e e h ö h e: 2333 m ü. d. M. Flu B g e b i e t: Mühldorferbach-Möll-Drau. Gr öße: Sehr kleines Hochgebirgsbecken von ungefähr $100 \mathrm{~m}$ Länge. Maximaltiefe: Nicht gemessen, schätzungsweise jedoch zu den tieferen Seen gehörig. Ge ologi e der Umgebung: Liegt in Urgestein.

Die folgende Speziesliste gründet sich auf die Untersuchung von Planktonnetzfängen, welche Herr Dr. K. Holdhaus im Sommer 1909 ausführte und von uns [PESTA 1911] bereits veröffentlicht wurde, sowie auf unsere Bearbeitung von neueren Fangproben, welche Herr Dr. A. PENTHER am 27. VIII. 1921 um $7^{\mathbf{h}}$ früh aus der Oberfläche des Sees sammelte. Es wurden insgesamt von uns nachgewiesen:

C o p e p o d a: Cyclops serrulatus (Fischer) [Pesta 1911 und 1921]. ? Cyclops vernalis Fischer [Pesta 1911]. Diaptomus sp. (iuvenis) [Pesta 1911]. Diaptomus bacillifer Koelbel [Pesta 1921].

Cla d o cera: Alona affinis (Leydig) [Pesta 1921]. Chydorus sphaericus (O. F. Müller) [Pesta 1921].

\section{Unterer Mühldorfersee.}

Fundortsnummer: 104. Se ehöhe: 2281 m ü. d. M. Grö ße: Ungefähr 400 Schritte lang. Maxi malt i e f e: Nicht ermittelt, gehört jedoch zu den tieferen Trichterseen. - $\mathrm{Z}$ o n e, g e 0 graphische Lage, Flubgebiet, Geologie der Umgebung wie vorhin beim Oberen Mühldorfersee (Nr. 103 ).

Die von Herrn Dr. K. Holdhaus im Sommer 1909 [Pesta 1911] und von Herm Dr. A. Penther am 16. VIII. 1921 aufgesammelten Planktonnetzfänge enthielten nach unserer Bestimmung folgende Formen:

Co pe pod a: Canthocamptus sp. [Pesta 1921].

Cla d o cera: Acroperus harpae Baird [Pesta 1921]. Alona affinis (LEYdiG) [PESTA 1921]. Chydorus sphaericus (O. F. MüLler) [PESTA 1911 und 1921].

\section{Münichsee.}

Fu n d orts n u m mer: 105. Z o n e: Nördliche Kalkalpen; Salzkammergut. Ge ographis ch e Lage: Oberösterreich, am Schaf$\Delta$ rehiv f. Hydrobiologie. Suppl.-Bd. III. 
berg zwischen Mondsee und Wolfgangsee gelegen. S e e h ö h e: $1262 \mathrm{~m}$ ï. d. M. F l u B g e b i et: Schwarzenbach-Ischl-Traun-Donau. C r ö B c: 0,03 $\mathrm{kn}^{2}$ Oberfläche; $200 \mathrm{~m}$ im größten Durchmesser. M a ximalti e f e: $34 \mathrm{~m}$ [Müllner 1896].

Für diesen Fundort erwähnt Langhans [1911] folgende Cladoceren:

Alona affinis (Leydig). Alona quadrangularis (O. F. MüLler). Ceriodaphnia pulchella SARS. Ceriodaphnia quadrangula var. hamata Lilljeborg [Langhans 1911 sub C. miülleri var. hamata].

\section{Mutterbergersee.}

Fundortsnummer: 106. Z 0 ne: Zentralalpen; Stubaiergruppe der Ötztaleralpen s. l. Ge o graph is ch e L a g e: Nordtirol, bei Ranalt im Stubaitale am Fuße der Hölltalerspitze (3282 m) gelegen. S e eh öh e: $2483 \mathrm{~m}$ ü. d. M. Flu Bge b i e t: Rutzbach-Sill-InnDonau. Grö Be: 300 Sehritte lang, 200 Schritte breit. M a xi malt i e f e nicht ermittelt, jedoch zu den tiefen Hochgebirgsseen gehörig. Ge ologische und hydrographisehe Daten: Das Becken liegt im Gneis und Hornblendeschiefer [BlaAs 1902]. Ufer ziemlich steil, Seeboden gegen die Mitte stärker geneigt abfallend. B o den besch affenheit steinig und sandig. Wa s serfarbe: Klar und tiefgrün. Wasserflor a: Kümmerliche Algenvegetation an Steinen. Oberflächliche Wassertemperatur bei Besonnung am 12. VIII. 1911 mit $13,6^{\circ} \mathrm{C}$ festgestellt. $\mathrm{Ch}$ e $\mathrm{m}$ is $\mathrm{c}$ h e $\mathrm{Zu}$ s a $\mathrm{m}$ me rsetzung des Wassers siehe Abschnitt II (Tabelle). Enthielt früher Saiblinge.

Pesta [1912], dessen Fänge vom 12. VIII. 1911 stammen, konnte nur folgende Formen nachweisen:

Co p e p o da: Cyclops sp. (iuvenis).

Cladocera: Alona affinis (Leydig). Chydorus sphaericus O. F. Müllek.

\section{Nassereithersee.}

F u n d or t s n u m mer: 107. Z o n e: Nördliche Kalkalpen; Nordtiroler Kalkalpen. Ge ogra phisehe Lage: Nordtirol, nördlich von Imst im Oberinntale. S e e h öh e: $840 \mathrm{~m}$ ü. d. M. Flu Bg e bi et: Gurglbach-Inn-Donau. Gr ö ß e: Kleines Wasser becken von vngefähr $120 \mathrm{~m}$ im Durchmesser. M a x i malt i e f e: $4 \mathrm{~m}$ (nach Dalla Torre [1913] und Geistbeck [1885] sogar nur 1,8 m). G e o l o g i s e h e u n d h ydrographische Daten: Der See soll seine Entstehung einer durch Erdbeben verursachten Bodensenkung verdanken oder durch 
Moränenbildung angestaut sein (vergl. dazu Dalla ToRre [1913]). Brehм [1907] hält ihn für einen Quellsee. Geistbeck [1885] maß am 14. VIII. 1881 eine Oberflächenwassertemperatur von $10,9^{\circ}$ C. - En t hält For ellen.

BREну [1907] erwähnt für diesen Fundort zwei Cladoceren, nämlich :

Alona affinis (LEYDiG) [sub Lynceus a.]. Chydorus sphaericus O. F. Müller.

\section{Neusiedlersee.}

Fu n d orts n u m mer: 108. Z o n e: Rand der Zentralalpen, Cetische Alpen. Ge o gra ph is che La ge: Ungarn, nahe der österreichischen Grenze südöstlich von Wien. S e e h öh e: $112 \mathrm{~m}$ ü. d. M. Flu Bge bi et: Rabnitz-Raab-Donau. Grö Be: $358 \mathrm{~km}^{2}$ Oberfläche; $35 \mathrm{~km}$ lang, $12 \mathrm{~km}$ breit. M a x i malt i e f e: $1-2 \mathrm{~m}$. Ge 0 logische und hydrographische Daten: Der Neusiedlersee liegt zwar schon außerhalb der Alpenzone, wir führen ihn jedoch gleich einigen bayerischen Voralpenseen des Vergleiches wegen hier an. Nach Halbfass [1913] wurde er neuestens aus der Reihe der Seen gestrichen, da sein Wasser durch Kunstkanäle abgeleitet wird; seit Beginn dieses Jahrhunderts hat er beständig an Wasseroberfläche verloren. Der Gr u n d dieses großen Seichtsees ist sehr schlammig, mineralische Laugensalze enthaltend. Ufer zum Teil von Weingärten und Wald umgeben. Enthält zahlreiche Fische und beherbergt viele Wasservögel.

Nach DadaY's [1891] Untersuchungen vom Juni 1890 kommen folgende Spezies vor:

Co p e p o d a: Cyelops viridis Jurine. Diaptomus spinosus Daday.

Cla d o c era: Diaphanosoma brachyurum (Liévin). Macrothrix laticornis (JuRINe). Moina brachiata (JuRINe). Scapholeberis mucronata (O. F. Müller). Sida crystallina (O. F. MülleR).

Nieder-Sonthofersee siehe Sonthofersee (Nr. 155).

\section{Niedertrumersee (Mattsee).}

Fu n d ortsn u m mer: 109. Z o ne: Nördliche Kalkalpen; Salzburger Kalkalpen. Ge ograph is che Lage: Salzburg, an der bayerischen Grenze westlich von Straßwalchen. Se eh öh e: $500 \mathrm{~m}$ ü. d. M. Flu Bge bi et: Mattig-Inn-Donau. Grö ße: $3,7 \mathrm{~km}^{2}$ Oberfläche; $4,1 \mathrm{~km}$ lang, $1,3 \mathrm{~km}$ breit. M a x i m al t i e f e: $40 \mathrm{~m}$. 
Geologisehe und hydrographische Daten: Ufer flach, Vegetation üppig. Nach Messungen von Micoletzky [1912], die vom 25. VIII. 1910 stammen, gehört dieser See zu den wärmsten Seen Salzburgs; die O berflä ch e $\mathrm{n}$ was sert e mperat u r betrug $19,1^{\circ} \mathrm{C}$, in $10 \mathrm{~m}$ Tiefe $15^{\circ} \mathrm{C}$, in $25 \mathrm{~m} \mathrm{7,8}{ }^{\circ} \mathrm{C}$ und in $40 \mathrm{~m}$ Tiefe (Grund) $7,4^{\circ} \mathrm{C}$.

Die eingehende Bearbeitung von Mrcoletzky [1912], dessen Fänge vom Juli und August des Jahres 1910 stammen, ermittelte folgende Spezies durch Planktonnetz und Kätscher, sowie Untersuchung von Sehlammproben:

Co pe poda: Cyclops albidus Jurine. Cyclops leuckarti Claus. Cyclops macrurus G. O. SARS. Cyclops serrulatus (FISCHER).

Clad ocera: Acroperus harpae BAIRD. Alona quadrangularis (O. F. MÜller). Alona rectangula G. O. SARS. Alona rectangula var. pulchra Stingelin. Alonella excisa (Fischer). Alonella exigua (LillueBORG). Alonella nana (BAIRD). Alonopsis elongata G. O. SARS. Camptocercus rectirostris (SchOEDLER). Ceriodaphnia pulchella G. O. SARS. Chydorus gibbus LILlJEBorg. Chydorus globosus BAIRD. Chydorus sphaericus O. F. MüLLER. Graptoleberis testudinaria (FISCHER). Peracantha truncata (O. F. MÜlLER). Sida crystallina (O. F. MÜLLER).

\section{Nussensee.}

Fu n d o r t s n u m mer: 110. Z o n e: Nördliche Kalkalpen; Salzkammergut. Ge $0 \mathrm{gr}$ a $\mathrm{ph}$ is e h e L a g e: Oberösterreich, westlich von Ischl. S e e h ö h e: $598 \mathrm{~m}$ ü. d. M. F l u B g e b i e t: Sofienbach-IschlTraun-Donau. Gr ö ß e: $0,09 \mathrm{~km}^{2}$ Oberfläche; $670 \mathrm{~m}$ lang, $210 \mathrm{~m}$ breit. Maximalti e fe: $15,5 \mathrm{~m}$, im Mittel $7,7 \mathrm{~m}$ (KeISSLer [1902] gibt irrtümlich $40 \mathrm{~m}$ Tiefe an). Hydrographische Daten: Die oberflächliche Wassertemperatur betrug nach einer Messung von Keissler am 31. VIII. $190117^{\circ}$ C. - Wasser „fast schwarz gefärbt".

Keissler [1902] und Langhans [1911] melden zusammen nur drei Formen:

Co p e poda: Diaptomus sp. [Keissler].

$\mathrm{Cl}$ a d o e e r a: Acroperus harpae (BaIRD) [Langhans sub A. bairdi]. Daphnia sp. [KeIssLeR].

Obersee beim Königssee.

F u n d o r t s n u m m e r: 111. Z o n e: Nördliche Kalkalpen; Berchtesgadnergruppe der Salzburger Kalkalpen. G e o g r a p h i s c h e L a g e: 
Bayern, südlich vom Königssee bei Berchtesgaden. S e e h ö h e: $610 \mathrm{~m}$ ü. d. M. Flu Bg e bi et: Ache-Salzach-Inn-Donau. Größ e: $1,3 \mathrm{~km}$ lang, $0,45 \mathrm{~km}$ breit.

Nur von Імноғ [1887] wird eine Gattung als vorkommend gemeldet, nämlich:

Daphnia sp.

\section{Vorderer Oberbergersee.}

Fu n d ortsnu m mer: 112. Z o n e: Nördliche Kalkalpen; Salzkammergut. Geographische Lage: Es ist uns nicht möglich gewesen, nähere Angaben über diesen Fundort, welchen ausschließlich LaNGHANs [1911] in seiner Bearbeitung der "Cladoceren aus dem Salzkammergut" erwähnt, aufzufinden. Die von ihm untersuchte Probe stammt vom 20. VIII. 1888 und enthielt folgende Formen:

Co p e p o d a: Cyclops sp.

$\mathrm{Cl}$ a d o c e ra: Acroperus harpae BAIRd. Alona affinis (Leydig). Bosmina sp. Chydorus sphaericus O. F. MÜLLER. Macrothrix rosea (JURINE).

\section{Ödensee.}

F u n d orts n u m mer: 113. Z o n e: Nördliche Kalkalpen; Salzkammergut. G e o gr a ph is c h e L a g e: Nordsteiermark, südwestlich von Kainisch bei Aussee. S e e h öh e: $764 \mathrm{~m}$ ü. d. M. F l u Bg e bi e t: Ödenseertraun-Traun-Donau. T i e fe: $25 \mathrm{~m}(20,86 \mathrm{~m})$. Gr öß e: $700 \mathrm{~m}$ lang, $300 \mathrm{~m}$ breit; $0,2 \mathrm{~km}^{2}$ Oberfläche.

Keissler [1907] sammelte an diesem See am 14. IX. 1906 Plankton; Langhans [1911] berichtet über die Cladoceren. Die beiden Autoren fanden folgende Formen:

C o p e p o d a: Cyclops sp. [Keissler].

Cla d o cera: Acroperus angustatus G. O. Sars [Langhans]. Acroperus harpae Baird [Langhans]. Alona affinis (Leydig) [LANGhans]. Alona costata G. O. Sars [Langhans]. Alona guttata G. O. Sars [Langhans]. Alona guttata var. tuberculata Thiébaud [Langhans]. Bosmina sp. [Keissler]. Daphnia sp. [Keissler]. Polyphemus pediculus (Linné) [Langhans]. Simocephalus vetulus (O. F. Müller) [Langhans].

\section{Offensee.}

Fundortsnummer: 114. Z o ne: Nördliche Kalkalpen; Salzkammergut. Ge o g r a ph is ch e L a g e: Oberösterreich, südlich vom Traunsee bei Ebensee. S e eh öh e: $651 \mathrm{~m}$ ü. d. M. Flu Bg ebiet: Offenseebach-Traun-Donau. Größ $\mathrm{e}: 0,61 \mathrm{~km}^{2}$ Oberfläche; un- 
gefähı $1000 \mathrm{~m}$ im größten Durchmesser. M a x i ma l t i e f e: $37,6 \mathrm{~m}$, im Mittel 19,5 m. - Utbrige Daten unbekannt.

Die Planktonfänge von IмноF [1885] (Fangdatum 28. VIII. 1884) und Keissier [1907] (Fangdatum23. VII. 1906) enthielten folgende Genera:

Co p e poda: Cyclops sp. [Imнof, Kerssler]. Diaptomus sp. [IMHOF, Keissler].

Cl a d o e er a: Bosmina sp. [Iмнof, Keissler]. Daphnia sp. [Keissler]. Daphnia longispina O. F. Müller [Iмhof]. Lynceus sp. [IMHOF].

\section{Ossiachersee.}

Fu n d o r t s n u m m r: 115. Z o n e: Zentralalpen; Grenze der Norischen Alpen gegen das Klagenfurter Becken. Ge o gr a ph is ch e L a g e: Kärnten, nordöstlich von Villach. S e e h öh e: $490 \mathrm{~m}$ ü. d. M. Fl u Bg e bi et: Tiebel-Seebach-Drau. Gr ö ß e: 10,75 $\mathrm{km}^{2}$ Oberfläche; über $10 \frac{1}{2} \mathrm{~km}$ lang, größte Breite $1 \frac{1}{2} \mathrm{~km}$. M a x i ma l t i ef e: $46,5 \mathrm{~m}$; jedoch ist fast die Hälfte des ganzen Seebeckens (gegen Nordosten) weniger als $10 \mathrm{~m}$ tief, auch die tiefere Wanne der südwestlichen Hälfte durchschnittlich nur 30-35 m tief. Nach HalbFass [1913] stellt der Ossiachersee ein durch Schuttkegel angestautes Diffluenzbecken dar. - Temperaturmessungen vergl. RichteR [1897, p. 185]. Enthält nach den Untersuchungen von $O$. HAEMPEL [i. l.] folgende $\mathrm{F}$ is $\mathrm{ch}$ e: Abramis brama, Alburnus lucidus, Barbus fluviatilis, Cyprinus carpio, Esox lucius, Gobio fluviatilis, Leuciscus cephalus, L. idus, Perca fluviatitis, Rhodeus amarus, Silurus glanis, Squalius cephalus, Tinca vulgaris, Trutta lacustris.

Nach den Untersuchungen von ImHoF [1890], STEuER [1897], Keissler [1905] und Brehm und Zederbauer [1905] kommen folgende Genera und Spezies vor:

Co pe poda: Cyclops sp. [Imнof, Keissler]. Cyclops albidus Jurine [Steuer]. Cyclops leuckarti Claus [Steuer, Brehm und ZederBAUER]. Cyclops macrurus SARS [STEUER]. Cyclops oithonoides var. hyalina Rehberg [Steuer]. Cyclops strenuus (Fischer) [STeuer, Brehm und Zederbauer]. Diaptomus sp. [Imhof, Keissler]. Diaptomus gracilis Sars [Steuer, Brehm und Zederbauer].

$\mathrm{Cl}$ a d o c e r a: Acroperus harpae BaIrd [Steuer sub A. leucocepha7us]. Alona affinis (Leydig) [Steuer]. Bosmina sp. [Imhof, Keissler]. Bosmina coregoni-longicornis (SCHOEDLER) [STEUER sub B. longicornis]. Bosmina coregoni-longispina Leydig [Brehm und Zederbauer]. Bosmina caregoni-longispina f. bohemica StIng. [STEuer sub B. bohemica]. Bosmina 
longirostris (O. F. Müller) [Brehn und Zeder baler]. Ceriodaphnia sp. [Imhof, Brehm und Zederbauer]. Daphnia sp. [Keissler]. Daphnia longispina var. cucullata SARs [Brehm und Zederbauer]. Daphnia longispina var. cucullata f. kahlbergensis SchoedLer [Iмноғ sub D. kahlbergensis, Steuer sub Hyalodaphnia jardini var. kahlbergensis]. Daphnia longispina var. hyalina LeYdig f. gracilis Hellich [Steuer sub D. hyalina var. gracilis]. Diaphanosoma brachyurum (LiÉvin) [Iмноғ, Steuer, Brehy und Zederbauer]. Leptodora kindtii (Focke) [Imhof, Steuer, Keissler, Brehy und Zederbauer]. Sida crystallina (O. F. Müller) [Iмнof].

Bei der bekannten Schwierigkeit einer exakten Bestimmung der verschiedenen Formen von Bosmina erscheint es uns richtig, aus der angeführten Aufzählung nur das Vorkommen der zwei Hauptspezies, B. coregoni BAIRD und B. longirostris (O. F. MüLLER), für den Ossiachersee als sicher nachgewiesen hervorzuheben. ohne auf die mehr oder weniger zweifelhafte Zuweisung zu besonderen Formenkreisen Rücksicht zu nehmen.

\section{Partnunersee.}

Fundortsnummer: 116. Z o n e: Nördliche Kalkalpen; Rhätikongebiet der Allgäueralpen. Ge ographis che Lage: Schweiz, nördlich von St. Anthönien, am Südhang der Sulzfluh gelegen. S e e h ö h e: $1874 \mathrm{~m}$ ü. d. M. F l u ß g e b i e t: DalvazzerbachLandquart-Rhein. Gr ö ß e: $450 \mathrm{~m}$ lang, $200 \mathrm{~m}$ breit. M a xi mal t i efe: Ungefähr $35 \mathrm{~m}$. Ge ologis che und hydrographi s ch e D a t e n: Das Becken liegt im Kalk (Trias- und Liaskalke). Von November bis Juni vereist. ZschokкE [1890] gibt für den Monat August 1889 eine $\mathrm{Oberflächenwassertemperatur}$ von $9,75^{\circ} \mathrm{C}$ im Mittel an, für die Monate Juli, August 1890 eine ebensolche von $11^{0} \mathrm{C}$ im Mittel.

Zschoкке [1890] und Schmeil [1893], welcher zum Teil die Bestimmungen des ersteren korrigierte, geben für diesen See folgende Spezies an:

C o p e p o d a: Canthocamptus rhaeticus Schmeil [Schmeir]. Canthocamptus zschokkei Schmeil [Schmeil]. Cyclops albidus Jurine [ZschoKke sub tenuicornis]. Cyclops strenuus (Fischer) [Zschoкке, SchmeiL]. Cyclops vernalis Fischer [Zschoкke sub elongatus, Schmeil]. Diaptomus bacillifer KоELBel [ZSCHOKKe].

Cla d o cera: Acroperus harpae Batrd [Zschoккe sub A. Teucocephalus]. Chydorus sphaericus O. F. MüLler [ZschoккE]. Daphnia 
longispina O. F. MÜLLER [ZschoKKe]. Rhynchotalona rostrata (Косн) [Zsснокке corr. ex Alona quadrangularis sub Lynceus rostratus].

Paßriachersee siehe Presseckersee (Nr. 126).

\section{Pfitscherjochseen.}

Fund ortsnummer: 117. Z o ne: Zentralalpen; Zillertaleralpengruppe der Hohen Tauern. Ge ograph is che Lage: Tirol, am Utbergang (Pfitscherjoch) vom Pfitschertal zum Zillertal gelegen. S e e h öh e: 2248 m ü. d. M. Flu Bg e b i et: Pfitscherbach-EisackEtsch. Größe: Sehr kleine Hochgebirgsseen von geringem Ausmaße; Tiefe nieht bekannt. Ge ol og is che und h ydrographische D a t e n: Die Seen liegen im Pfitscher Schiefergebiet; entstanden durch Gletschererosion [BöHM 1886, Dalia ToRRe 1913].

In diesen beiden engbenachbarten Hochgebirgsbecken, welche am 18. VII. 1901 von Brehm und Zederbauer [1904] auf ihren Planktongehalt untersucht wurden, fanden die genannten Autoren eine einzige. Spezies, nämlich:

Cyclops serrulatus (FISCHER).

\section{Piazzesee (Lago delle Piazze).}

Fundortsnummer: 118. Z o n e: Südliche Kalkalpen; Südtiroler Dolomiten. Ge ogra ph is che Lage: Südtirol, Bezirk Trient, nördlich vom Caldonazzosee im „Val di Pine“ gelegen. S e e höh e: 1013 m ü. d. M. Flu Bge biet: Regnanabach-Avisio-. Etsch. Größe: $1000 \mathrm{~m}$ lang, $300 \mathrm{~m}$ breit; 0,22 $\mathrm{km}^{2}$ Oberfläche. Maximaltiefe: $19 \mathrm{~m}$. Ge ologisehe und ydrographi$\mathrm{s}$ e h e Dat e n: Liegt im Porphyrgebiet. Am 21. VIII. 1897 maß LarGaIolli [1898] eine $\mathrm{Oberfläch}$ enwasserte mperatur von $18,8^{\circ} \mathrm{C}$; von Ende Dezember bis anfangs April vereist. Enthält F i s c h e (Barbe, Hecht, Karpfen, Rotfeder, Schleie u. a.).

Buffa [1902], welcher diesen Fundort am 10. VIII. 1901 untersuchte, meldet das Vorkommen folgender Formen:

Co pe poda: Canthocamptus minutus Claus.

Cl a d o c e ra: Bosmina longirostris-cornuta JURINE. Diaphanosoma brachyurum (Liéviv).

\section{Piburgersee.}

Fundortsnummer: 119. Z o ne: Zentralalpen; Ötztaleralpen. Ge ographische Lage: Tirol, bei Sautens im Ötztale. 
S e ehöh e: 915 m ü. d. M. Flu Bg e bi et: Ötztalerache-InnDonau. Größ B : $134000 \mathrm{n}^{2}$ Oberfläche; $800 \mathrm{~m}$ lang, $250 \mathrm{~m}$ breit. Maximaltiefe: $30 \mathrm{~m}$. Ge ologische und h ydrographis eh e Daten: Nach Dalla Torre [1913] füllte ein Bergsturz das Tal auf, sperrte die Talrinne gegen Osten ab und bildete unter Mitwirkung des im Westen vorliegenden Felsriegels das Seebecken. Brenм und Zederbauer [1904] maßen am 20. VIII. 1901 eine O b e r f l ä e h e n was sertem perat ur von $18^{\circ} \mathrm{C}$, am 23. XII. 1901 eine von $4^{0} \mathrm{C}$ in $5 \mathrm{~m}$ Tiefe. Enthält $\mathrm{Fi}$ is $\mathrm{che}$ : Aitel, Barsch, Rotfeder.

Nach Bremm und Zederbauer [1904] finden sich im Plankton folgende Spezies:

C o p e p o d a: Cyclops albidus Jurine.

$\mathrm{Cl}$ a d o c e ra: Bosmina longirostris (O. F. MüLler). Ceriodaphnia quadrangula (0. F. MüLLER) f. typica. Daphnia longispina var. hyalina LEYDIG.

\section{Pillersee.}

Fundortsnummer: 120. Z o ne: Nördliche Kalkalpen; Waidringergruppe der Salzburger Kalkalpen. Ge ograph is eh e L a g e: Nordtirol, bei St. Ulrich, südlich von Waidring, nahe der bayrischen Grenze. S e e h öh e: $834 \mathrm{~m}$ ü. d. M. Fl u Bg e bi e t: PillerSaalach-Salzach-Inn-Donau. Gr ö B e: $1,8 \mathrm{~km}$ lang, $300 \mathrm{~m}$ breit. Maximaltiefe: Nicht ermittelt. Ge ologisehe und hydrographische Daten: Liegt im Gebiet erzhaltigen Kalkgesteins. Das Becken gehört zu den Stauseen (durch Schuttkegel). En thält a n F is chen: Aitel, Forelle, Koppe, Pfrille, Schmerle und Steinpitzger.

BREHM [1907] hat im Pillersee nachgewiesen:

Co pe p o da: Cyclops serrulatus (FISCHER).

$\mathrm{Cl}$ a d o c e r a: Alonopsis elongata G. O. SARs. Chydorus sphaericus O. F. MüLLER. Eurycercus lamellatus (0. F. MüLleR). Graptoleberis testudinaria (Fischer).

\section{Plansee.}

Fundortsnummer: 121. Z o ne: Nördliche Kalkalpen; Ammergauergebiet der Nordtiroler Kalkalpen. Ge $0 \mathrm{gr}$ a $\mathrm{ph}$ is $\mathrm{ch}$ e L a g e: Nordtirol, östlich von Reutte an der bayrischen Grenze. S e e h öh e: 975 m ü. d. M. Flu ßg e b i et: Planseeache-Lech-Donau. Größe: $5 \mathrm{~km}$ lang, $1 \mathrm{~km}$ breit. M a xi maltiefe: $75 \mathrm{~m}$ [nach Geistbeck 1885 und Dalla Torre 1913]. Ge ologis ehe und h y dro gra ph i s e h e D a t e n: Liegt im Gebiet des Hauptdolomites. 
Nach Böнm [1886] gehört der Plansee zu den durch Terrassenschotter gebildeten Abdämmungsseen. Ufer bewaldet. Wa ss erfä r bu ng dunkelgrün. F is ch b e st and: Karpfen, Pfrille, Ranke, Saibling, Secforelle.

Iмноғ [1885̃], welcher seine Planktonfänge am 16. VIII. 1884 ausführte, konnte folgende Arten nachweisen:

Co pe poda: Cyclops sp. Diaptomus sp. (iuvenis).

Cl a d o cera: Bosmina sp. Bythotrephes longimanus Leydig. Daphnia longispina O. F. MülLER. Leptodora kindtii (Focke).

Plaschischensee siehe Keutschachersee (Nr.60).

\section{Oberer Plenderlesee.}

Fundortsnu m mer: 122. Z o ne: Zentralalpen; Ötztaleralpen, Sellrainergruppe. Ge o g r a ph is che Lage: Tirol, im sog. Kühtai, dem Übergang vom Sellraintal in das Ötztal gelegen. S e e h ö h e: $2327 \mathrm{~m}$ ü. d. M. [nach Dalla Torre 1913]. Fl u B.g e b i e t: Finstertalerbach-Stuibenbach-Ötztalerache-Inn. Gr ö B e: Kleines, langgestrecktes Hochgebirgsbecken von ungefähr gleicher Ausdehnung wie der Untere Plenderlesee. Ti e fe: Unbekannt. Ge o lo g i s c h e und hydrographisehe Daten: Liegt im Gneis. Enthält S a iblinge [nach Dalla Torre 1913].

Brehm und Zederbauer [1904], welche diesen See am 21. VIII. 1901 auf seinen Planktongehalt prüften, fanden hier ke i n e n t i e r i s e h e n B e w o h n er; vergl. dazu unsere Angaben über den Unteren Plenderlesee (Nr. 123).

\section{Unterer Plenderlesee.}

F u n d ortsnum mer: 123. Z o n e: Zentralalpen; Ötztaleralpen, Sellrainergruppe. Ge $0 \mathrm{gr}$ a $\mathrm{ph}$ i s c h e L a ge: Tirol, am Übergang vom Sellraintal in das Ötztal gelegen (sog. Kühtai). S e e h öh e: ca. $2250 \mathrm{~m}$ ü. d. M. Fl u B g e b i e t: Finstertalerbach-StuibenbachÖtztalerache-Inn-Donau. Gr ö ße: Ungefähr 230-250 Schritte lang und etwa 80 Schritte breit. Maximaltiefe: Unbekannt. Geologische und hydrographische Daten: Liegt im Gneis. Ufer allseits ziemlich steil, steinig. S e e b o d e n mit großen Steinblöcken belegt, dazwischen feinsandig. Alg e nflor a spärlich entwickelt, höhere Wasserpflanzen fehlen. Am 27. VIII. 1914 wurde von uns eine $\mathrm{Oberfläehenwassertemperatur}$ von $7,3^{\circ} \mathrm{C}$ gemessen (gleichzeitige Lufttemperatur $9^{\circ} \mathrm{C}$ im Schatten). Wasser klar, 
von grüner Färbung. Der See enthielt Saiblinge. - Che $\mathrm{m}$ is che Zus ammensetzung des Seewassers siehe Abschn. II (Tabelle).

Auch in diesem See fand Brehy und Zederbauer [1904] am 21. VIII. 1901 keinen tierischen Bewohner. Diese für die beiden Plenderleseen gelţende auffällige Angabe veranlaßte uns zu einer Nachuntersuchung [Pesta 1915] des Unteren Seebeckens. Es sei hervorgehoben, daß wir bei dieser Gelegenheit das Vorkommen von Fischen (Saiblingen) selbst beobachtet kaben. Die rom Ufer aus mit dem Planktonnetz ausgeführten Fänge (27. VIII. 1914) enthielten neben Hautresten ron Insektenlarven und einem Borstenwurm folgende Entomostraken:

Co p e poda: Cyclops sp. (iuvenis). Cyclops serrulatus (Fischer).

Cla d o c e r a: Chydorus sphaericus O. F. Müller.

Dementsprechend dürften auch die Verhältnisse im Oberen Plenderlesee andere sein als wie Brehm und Zederbauer [1904] gemeldet haben, deren Ergebnis der Untersuchung zweifellos auf einem Irrtum beruht.

\section{Unterer Pochartsee (= Bockhartsee).}

F u n d o r t s n u m m e r: 124. Z o n e: Zentralalpen; Hohe Tauern. Ge 0 gra ph is ch e La ge: Salzburg, Naßfeldgraben bei Gastein. S e e h öh e: $1846 \mathrm{~m}$ ü. d. M. Fl u B g e b i e t: NaßfeldgrabenbachGasteinerache-Salzach-Inn-Donau. Gr ö ß e: $840 \mathrm{~m}$ lang, $380 \mathrm{~m}$ breit. Maximalt i e fe: $46 \mathrm{~m}$. Ge o logie der Umge bung: Der See liegt im Granit. [Vergl. dazu FugGer 1911.] Nach Böнм [1886] durch einen Bergsturz angestaut.

Brenм [1907] erwähnt diesen von ihm besuchten Fundort ohne Angaben über die Entomostrakenfauna.

\section{Pragsersee (= Pragserwildsee).}

Fundortsn u m mer: 125. Z o n e: Südliche Kalkalpen; Südtiroler Dolomiten. Ge o $\mathrm{gr}$ a $\mathrm{ph}$ is ch e L a g e: Südtirol, südwestlich von Niederndorf im Pustertale. S e e h öh e: $1496 \mathrm{~m}$ ü. d. M. Flu ßg e b i e t: Pragserbach-Rienz-Eisack-Etsch. Gr ö ß e: ca. $1200 \mathrm{~m}$ lang, $300 \mathrm{~m}$ breit. Ma x i malt i e f e: Unbekannt. G e o log i s c e u nd h y drogra phis ch e Dat e n: Liegt im Dolomitgebiet. Nach Dalla Torre [1913] durch Schuttkegel gestaut. Ufer steil, besonders am Südrand. Brehm und Zederbauer [1905] maßen am 4. VIII. 1903

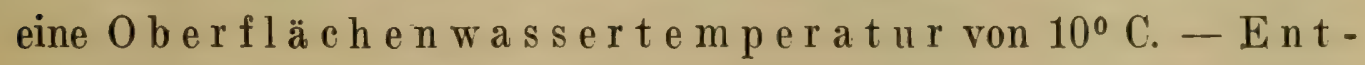
$\mathrm{h}$ ält: Forellen und Saiblinge. 
Das Crustaceenplankton dieses Sees setzt sich nach den Untersuchungen ron BREHM und Zederbauer [1905] aus folgenden Arten zusammen:

C o p e p o d a: Cyclops strenuus Fischer.

Cl a d o cera: Bosmina longirostris-cornuta JuRive. Daphnia longispina var. hyalina LEYDIG f. foreli BURCKH.

\section{Presseckersee (= Paßriachersee).}

F u n d ortsnum mer: 126. Z o n e: Südliche Kalkalpen; Gailtalergruppe der Karnischen Alpen. Ge ographische Lage: Kärnten, östlich von Hermagor im Gailtale. S e e h ö h e: 567 m ü. d. M. F 1 u B g e b i e t: Fellbach-Gail-Drau. Gr ö B e: $1000 \mathrm{~m}$ lang, $600 \mathrm{~m}$ breit. Tiefe: Nicht ermittelt. Ge ologisehe und hydrographische Daten: Nach Frech [1903] ist die Bildung des Presseckersees auf Unregelmäßigkeit in der Wirkung von Erosion und Stauung zurückzuführen, eine Erscheinung, die in den Alpen selten sein soll.

Steuer [1901] und Brehy und Zederbauer [1905] fanden hier folgende Planktonvertreter:

Co p e p o da: Cyclops sp. (iuvenis) [Brehm und Zederbauer]. Diaptomus graciloides Lilljeborg [Steuer, Brehu und Zederbauer].

$\mathrm{Cl}$ a d o c e r a: Bosmina longirostris (O. F. MÜLLER) [BREHM und Zederbauer]. Ceriodaphnia sp. [Brehm und Zederbauer].

\section{Raiblersee.}

Fu n d o r t s n u m m e r: 127. Z o n e: Südliche Kalkalpen; Julische Alpen. Ge o g r a ph i s c h e L a g e: Südkärnten, bei Raibl am Predilpaß. S e e h öh e: $990 \mathrm{~m}$ ü. d. M. Fl a B g e bi e t: Schlitza-GailitzGail-Drau. Gr ö Be: $2 \mathrm{~km}$ lang, 0,4 km breit.

Die spärlichen Angaben über die Entomostrakenfauna dieses Sees. beziehen sich ausschließlich auf Planktonformen; Langhans [1905], der am 20. VIII. 1903 und im Mai 1904 fischte, und BREHM [1906] berichten lediglich über das Vorkommen von zwei $\mathrm{Cl}$ a d o e e r e n, nämlich:

Bosmina sp. [Langhans 1905] und Polyphemus pediculus (Linné) [BREHM 1906].

\section{Reintalersee.}

F u n d o r t s n u m mer: 128. Z o n e: Nördliche Kalkalpen; Nordtiroler Kalkalpen. Ge $0 \mathrm{graph}$ is e h e L a ge: Nordtirol, nördlich von Rattenberg im Unterinntale. S e e h öh e: $558 \mathrm{~m}$ ü. d. M. F.l u B - 
g e b i e t: Achenrainerbach-Brandenbergerache-Inn-Donau. Gr öße: 27,5 ha Oberfläche; ca. $1100 \mathrm{~m}$ lang, $380 \mathrm{~m}$ breit. M a x i ma l t i e f e: $10,5 \mathrm{~m}$. Geologisehe und hydrographisehe Daten: Entstanden durch Schuttkegel von Wildbächen und Muhrbruch [nach Böнм 1886]; liegt in glazialem Schutt. Ufer flach und stark verschilft; zahlreiche Seerosen. Enthält a n F is ch en: Aitel, Hecht, Pfrille, Rotfeder.

Brehm [1902 und später 1907] meldet für diesen Fundort folgende $\mathrm{Cl}$ a d o cer en:

Acroperus harpae BAIRD. Diaphanosoma brachyurum (LiÉvix). Eurycercus lamellatus (O. F. MüLleR) [BRenm 1902]. Graptoleberis testudinaria (Fischer). Peracantha truncata (O. F. Mëller) [Brehm 1902 sub Pleuroxus tr., Brenm 1907]. Pleuroxus trigonellus (O. F. MülLer). Sida crystallina (O. F. Müller).

\section{Reithersee.}

Fun d o r t s n u m mer: 129. Z o n e: Zentralaipen; Salzburger Schieferalpen. Ge o gra $\mathrm{ph}$ is ch e L a g e: Nordtirol, bei Brixlegg im Unterinntale. S e e h ö h e: 657 m ü.d. M. Fl u B g e b i e t: Inn-Donau; der Abfluß des Sees ist unterirdisch gegen Norden [Dalda Torre 1913]. Gr ö B e: Sehr kleines Wasserbecken mit einem Höchstdurchmesser von 150 m. Tiefe: Unbekannt. Geologisehe und hydro$\mathrm{graph}$ ische Daten: Der Reithersee ist eine kesselförmige Vertiefung auf einer glazialen Terrasse [Dalla TorRe 1913].

Brenм [1907] fand in seinen rom 23. VIII. stammenden Netzfängen folgende Formen:

Co p e p od a: Cyclops leuckarti Claus. Cyclops strenuus Fischer.

Cl a d o e e ra: Alona costata Sars [Brehm sub Lynceus c.]. Bosmina sp. (iuvenis). Chydorus sphaericus O. F. MüLlER. Scapholeberis mucronata (O. F. MÜLLER).

\section{Revinesee (Lago di Revine).}

Fundortsnummer: 130. Z o n e: Südliche Kalkalpen; Venetianer Alpen. Geographische Lage: Norditalien, Bezirk Treviso, westlich von Vittorio gelegen. S e e h öh e: $235 \mathrm{~m}$ ü. d. M. Flu Bgebi et: Marengo-Piave (Adria). Grö Be: $3000 \mathrm{~m}$ lang, $1000 \mathrm{~m}$ breit. M a xi malti e f e: $15 \mathrm{~m}$. Ubrige Daten nicht bekannt.

Die folgende Speziesliste gründet sich auf Fangergebnisse, welche vom 8. X. 1879 stammen und von Pavesi [1884] veröffentlicht wurden. 
C o p e p o da: Cyclops viridis Jurine [Pavesi sub C. brevicornis]. Diaptomus castor (JURINE).

Cl a d o e e r a: Ceriodaphnia quadrangula (O. F. MüLler). Daphnia longispina var. hyalina LeYdig f. galeata SARS [PAVESI sub D. galeata]. Diaphanosoma brachyurum (LiÉvin). Leptodora kindtii (FockE).

\section{Rissersee.}

Fu n d ortsnu m mer: 131. Z o n e: Nördliche Kalkalpen; Wettersteingruppe der Nordtiroler Kalkalpen. Ge o g r a p h i s c h e L a g e: Bayern, bei Garmisch-Partenkirchen nahe der Tiroler Grenze. S e e höh e: $785 \mathrm{~m}$ ü. d. M. Flu Bg e bi et: Loisach-Isar-Donau. Gr ö B e: Langgestrecktes, schmales Wasserbecken von ca. $500 \mathrm{~m}$ Länge. T i e fe: Unbekannt.

Brenm [1907] fand im Juniplankton folgende Cl a d o c e r e n:

Bosmina longirostris f. cornuta JURINE. Ceriodaphnia pulchella G. O. SARS.

\section{Röthelsee.}

Fu n d o r t s n u m mer: 132. Z o n e: Nördliche Kalkalpen; Salzkammergutgebiet. G e $0 \mathrm{gra} \mathrm{ph}$ is c h e L a g e: Oberösterreich, östlich vom Südende des Traunsees gelegen. Flu Bg e b i et: Traun-Donau. S e e h öh e: Ungefähr $1000 \mathrm{~m}$ ü. d. M. Gr ö ß e: Sehr kleines, unterirdisches Becken am Fuße des Erlakogels. Nähere Daten unbekannt.

Keissler [1907] fand hier keine Organismen. Die Fundstelle sei nur der Vollständigkeit wegen genannt, nachdem Iмноғ [1885] in diesem See einen Cyclops gefangen hat (28. VIII. 1884), welchen er als möglicherweise mit der unsicheren Art $C$. $m$ a $g n$ i c e $p s$ LILLJE BORG identisch hielt.

\section{San Danielesee (Lago di S. Daniele).}

Fu n d o rtsnummer: 133. Z o n e: Südliche Kalkalpen; Venetianer Alpen (Südostgrenze). Ge ograph is che Lage: Norditalien, Bezirk Udine, bei San Daniele unweit des linken Tagliamentoufers gelegen. S e e h öh e: ca. $270 \mathrm{~m}$ ü. d. M. F l u ß g e b i e t: Tagliamento (Adria). Grö Be: $700 \mathrm{~m}$ lang. M a x i malt i e f e: $9 \mathrm{~m}$. Ge o $\mathrm{l} 0 \mathrm{~g}$ i s che und h y d r o graphis che Date n: HalbFass [1913] schreibt über diesen See: „Gehört zum Amphitheater der Moränen des Tagliamento, das durch den ehemaligen Karnischen Gletscher entstanden ist. Auch er ist im raschen Erlöschen begriffen ... . - Enthält an Fischen: Anguilla, Barbus, Cottus, Leuciscus (drei Spezies), Tinca. 
Senva [1890], dessen Fänge am 27. V. 1889 ausgeführt wurden, meldet folgende Spezies:

C o p e p o d a: Cyclops diaphanus Fischer [sub C minutus]. Diaptomus gracilis SARS var. carnicus SENNA.

Cla d o c e r 2: Bosmina longirostris (O. F. MülLer).

\section{Santa Crocesee (Lago di S. Croce).}

F u n d orts n u m mer: 134. Z o n e: Südliche Kalkalpen; Venetianer Alpen. Ge o graph is ch e Lage: Norditalien, östlich von Belluno. S e e h öh e: $362 \mathrm{~m}$ ü. d. M. Fl u $\mathrm{Bg}$ e b i e t: Raital-Piave (Adria). Gr ö ß e: 4,6 km² Oberfläche; $4 \mathrm{~km}$ lang, $3 \mathrm{~km}$ breit. M a ximaltiefe: $32 \mathrm{~m}(34 \mathrm{~m})$. Geologische und hydrogr a ph i s e h e D a t e n: HalbFass [1913] verlegt die Entstehung des Sees in die Glazialzeit; er konnte sich nur erhalten, weil er abseits des großen Gletscherstromes lag, als dieser sein Bett aufschüttete. Die Speisung soll hauptsächlich durch Quellen am Grunde bestehen; vermutlich ist er unterirdisch mit dem Lago morto verbunden.

Über die Copepoden und Cladoceren des Santa Crocesees berichtet Pavesi [1884].

C o p e p o d a: Cyclops serrulatus Fischer. Cyclops viridis Jurine [sub C. brevicornis].

Cl a d o ce ra: Diaphanosoma brachyurum (LiÉviv) [sub Daphnella b.]. Leptodora kindtii (Focke). Sida crystallina (O. F. Müller).

\section{Santa Massenzasee (Lago di S. Massenza).}

F u n d orts n u m mer: 135. Z o n e: Südliche Kalkalpen; Etsclbuchtgebirge. Ge ographische Lage: Südtirol, südlich von Vezzano bei Trient. S e eh öh e: $250 \mathrm{~m}$ ü. d. M. Flu Bgebiet: Sarca-Mincio-Po. Gr ö B e: Ungefähr $1000 \mathrm{~m}$ lang, $500 \mathrm{~m}$ breit. Ma a imalti ef e: Unbekannt. Enthält nach Dalla Torre [1913] folgende Fis che: Aal, Bar be, Forelle, Hecht, Karpfen, Koppe, Rotfeder, Schleie u. a.

Largatolli [1898] meldet das Vorkommen folgender Entomostraken:

C o p e p o da: Cyclops fuscus Jurine. Cyclops serrulatus Fischer.

$\mathrm{Cl}$ a d o e e r a: Bosmina longirostris (O. F. Müller). Ceriodaphnia quadrangularis (0. F. MüLLER). 


\section{Santosee (Lago Santo).}

F u n d orts n u m mer: 136. Z o n e: Südliche Kalkalpen; Etschbuchtgebirge. Ge $0 \mathrm{graph}$ isch e Lage: Südtirol, westlich von Lavis an der Etsch. S e e höh e: $707 \mathrm{~m}$ ü. d. M. (nach Dalla Torre [1913] $746 \mathrm{~m}$ ü. d. M.). Flu Bg e bi e t: Etsch. Grö Be: $0,11 \mathrm{~km}^{2}$ Oberfläche; $780 \mathrm{~m}$ lang, $150 \mathrm{~m}$ breit. M a x i mal t i e f e: $13 \mathrm{~m}$. Ge 0 $\mathrm{log}$ ische und hydrographisehe Daten: Liegt im Kalkgestein. W as serfarbe intensiv grün, von geringer Transparenz. LARGAIOLLI [1906/07] maß am 28. VII. eine $O \mathrm{~b}$ e $\mathrm{r} f \mathrm{l}$ ä c h e $\mathrm{n}$ w a s s e rt e m peratur von $22^{\circ}$ C. F isch best and: Hecht, Karpfen, Koppe, Rotfeder, Schleie, Weibfisch u. a.

Nach den Untersuchungen von Largaiolli [1906/07] beherbergt der See folgende Arten:

C o p e p od a: Cyclops strenuus Fischer.

$\mathrm{C} 1$ a d o c e r a: Acroperus angustatus G. O. SARs. Bosmina longirostris (0. F. Müller). Ceriodaphnia pulchella G. O. SARS. Diaphanosoma brachyurum (LiÉvin). Leptodora kindtii (Focke). Peracantha truncata (O. F. Müller). Sida crystallina (O. F. Müller).

\section{Saualmsee ${ }^{1}$ ).}

(Bisher nicht veröffentlicht.)

Fundortsnummer: 137. Z o ne: Zentralalpen; Norische Alpen. G e o g r a ph i s e h e L a g e: Ostkärnten, nächst der höchsten Erhebung des Saualpenzuges zwischen dem Lavanttal und Görschitzbach. S e ehöh e: ca. 1950 m ü. d. M. Flu Bg e bi et: Seitenbach der Lavant-Lavant-Drau. Gr ö ß e: Sehr kleines und tümpelartiges, aber perennierendes Wasserbecken; Seichtsee. Übrige Daten unbekannt.

Die Planktonnetzfänge, welche Herr Dr. K. Holdhaus hier am 14. VII. 1920 ausführte, wurden von uns untersucht und enthielten folgende Formen:

Co p e p oda: Cyclops sp. (iuvenis). Diaptomus tatricus WrerZEJSKI.

Cladocera: Alona affinis (Leydig). Chydorus sphaericus (O. F. MüLler). Daphnia longispina var. longispina (LEYDIG).

1) Dieser Fundort ist nicht zu verwechseln mit dem von STEUER [1897] erwähnten Tümpel unterhalb der Spitze des Zirbitzkogels in $1800 \mathrm{~m}$ ü. d. M. Der Zirbitzkogel ist die höchste Erhebung der Seetaleralpen (Steiermark) auf der sog. Weiten Alm (nicht Saualpe wie STEUER irrtümlich schreibt!). 


\section{Saureggersee.}

(Bisher nicht veröffentlicht.)

Fund ortsnummer: 138. Z o ne: Zentralalpen; Norische Alpen. Ge ographis che Lage: Nordkärnten, am Saureggnock im Königstuhlgebiet gelegen. S e e h öh e: ca. $2000 \mathrm{~m}$ ü. d. M. F l u fg e b i e t: Kremsgrabenbach-Lieser-Drau. Gr ö Be: ca. $100 \mathrm{~m}$ lang und $50 \mathrm{~m}$ breit. Geologisehe und hydrographische D a t e n: Das Wasserbecken liegt im Karbonischen Quarzkonglomerat. Moränensee. Ufer zum Teil flach und mit Schilfgras bewachsen; Secboden schlammig.

In den von Herrn Dr. K. Holdhaus Mitte August 1921 gesammelten Planktonnetzfängen waren folgende Spezies enthalten:

Co p e p o d a: Cyclops vernalis (FISCHER).

Cla d o e era: Alona affinis (Leydig). Daphnia longispina rar. longispina LEYDIG.

\section{Schlickersee.}

Fundortsnummer: 139. Z o ne: Zentralalpen; Stubaiergruppe der Ötztaleralpen im weiteren Sinne. Ge ographische L a ge: Tirol, im Gebiete des Hohen Burgstall (2613 m, Stubaital) gelegen. S e e höh e: ca. $2500 \mathrm{~m}$ ü. d. M. Flu Bg e b i e t: SeebachAlpeinerbach-Ruez-Sill-Inn-Donau. Gr ö Be: 125 Schritte lang, 70 Schritte breit. II a i maltiefe: Nicht genau ermittelt, jedoch nur einige Meter. Geologische $u$ nd hydrographische D a te n: Das Becken liegt im Kontaktgebiet von Schiefer und Kalk (Triaskalke über Glimmerschiefer). Ufer an der Nordseite ein steil geneigtes Kaar (Geröllhalde) bildend, im übrigen seicht. S e e b o d e n mit Schieferblöcken belegt, der feinsandige Detritus deutlich glimmerig. Wasserflor a sehr spärlich (Algen) entwickelt, höhere Wasserpflanzen fehlen. Am 13. VIII. 1910 wurde von uns eine O b e r f 1 ä c h e nwas sertemperatur von $5,6^{\circ} \mathrm{C}$ gemessen (die Temperatur des einzigen Schmelzwasserzuflusses betrug nur $1,3^{\circ} \mathrm{C}$ ). Der See friert angeblich erst Ende Juli, anfangs August auf. - Fisch e w ur den von uns nicht beobachtet.

Es wurden von uns [PESTA 1911] lediglich folgende Formen nachgewiesen:

C o p e p o d a: Cyclops vernalis Fischer.

Cladocera: Alona affinis (Leydig). Chydorus sphaericus O. F. MÜLler. 


\section{Schliersee.}

Fundortsnummer: 140. Z o ne: Nördliche Kalkalpen; Nordtiroler Kalkalpen. Ge $0 \mathrm{gr}$ a $\mathrm{ph}$ i s ch e L a ge: Südbayern, südwestlich von Rosenheim und östlich vom Tegernsee gelegen. S e e h öh e: $768 \mathrm{~m}$ ü. d. M. Fl u Bg e b i e t: Schlierach-Mangfall-InnDonau. Größe: $2,6 \mathrm{~km}$ lang, $1,1 \mathrm{~km}$ breit. M a ximalti efe: $39 \mathrm{~m}$ [GeIST BECK 1885].

Iмноғ [1887] und Steuer [1901] geben für den Schliersee nachfolgende Copepoden und Cladoceren an:

C o p e p o d a: Cyclops sp. [Імноғ] Diaptomus sp. [Імноғ]. Heterocope weismanni Iмноғ [STEUER].

Cla d o e e ra: Bosmina sp. [Імноғ]. Daphnia longispina var. hyalina Leydig [ImноF sub D. hyalina]. Diaphanosoma brachyurum (LiÉvin) [Iмно sub Daphnella br.]. Leptodora kindtii (Fоске) [Імноғ sub Lept. hyalina].

\section{Schwansee.}

Fund ortsnummer: 141. Z o ne: Nördliche Kalkalpen; Nordtiroler Kalkalpen. Ge o graphische Lage: Südbayern, nächst der Tiroler Grenze, südöstlich von Füßen (bei Hohenschwangau). S e e höh e: $790 \mathrm{~m}$ ü. d. M. Flu Bg e b i e t: Lech-Donau. Gr ö ße: ca. $800 \mathrm{~m}$ lang, größte Breite $300 \mathrm{~m}$. M a x i m a l ti e f e: Unbekannt. Ebenso fehlen übrige Daten.

Імноғ [1887] determinierte aus dem August-Septemberplankton drei $\mathrm{Cl}$ a d o c ere $\mathrm{n}$, nämlich:

Bosmina sp. Daphnia sp. Diaphanosoma brachyurum (LIÉvin).

\section{Schwarzsee bei Kitzbühel.}

Fundortsnu m mer: 142. Z on e: Zentralalpen; Kitzbühler Schieferalpen. Ge ograph is che Lage: Osttirol, bei Kitzbühel. S e ehöhe: $786 \mathrm{~m}$ ü. d. M. Flu Bg e bi et: Seebach-ReinacheGroße Ache-Inn-Donau. G r ö B e: Größte Länge ca. $600 \mathrm{~m}$; ungefähr 8 ha Oberfläche. Maxi malti efe: Unbekannt. Ge ologis che und hydrographische Daten: Liegt im Gebiete der Grauwackenschiefer. Nach Dalla ToRRE [1913] ein Moränensee mit eisenhältigem Wasser. Der Name Schwarzsee bezieht sich auf die $\mathrm{dunkle}$ Wasserfärbung infolge des moorigen Grundes (Moorwasserbad mit $20-27^{\circ} \mathrm{C}$ ). Ufer versumpft und flach; Wasserflora, insbesondere Planktondiatomaceen reich entwickelt. Enthält zahlreiche 
F i s c h e: Aitel, Barsch, Brachsen, Hecht, Karpfen, Laube (Alburnus), Pfrille, Rotfeder, Schleie, Schmerle, Steinpitzger [Dalla Torre 1913].

Dieser See wurde von Zacharias [1902] und von BREhm [1907] in bezug auf Planktoncrustaceen untersucht; demnach kommen hier vor:

Co p e poda: Cyclops leuckarti Claus [Brehm]. Diaptomus sp. [Zacharias].

Cl a d o e e r a: Alonella nana (BAird) [Zacharias sub A. pygmaea]. Bosmina longirostris (O. F. Müller) [Zacharias, Brehm]. Bosmina longirostris cornuta JURINe [Zacharias]. Ceriodaphnia pulchella G. O. SARS [Zacharias]. Diaphanosoma brachyurum (LiÉvin) [Zacharias].

\section{Schwarzsee am Schafberg (Schwarzensee).}

Fundortsnummer: 143. Zone: Nördliche Kalkalpen; Salzkammergutgebiet. Ge ogra ph is ch e Lage: Oberösterreich, nordwestlich von St. Wolfgang am Wolfgangsee, am Fuße des Schafberges. S e e h öh e: $711 \mathrm{~m}$ ü. d. M. F l u Bg e bi e t: SchwarzenbachIschl-Traun-Donau. Gr ö B e: 0,48 km² Oberfläche; $1430 \mathrm{~m}$ lang, $400 \mathrm{~m}$ breit. Maximalti e fe: $54 \mathrm{~m}$; im Mittel $29,6 \mathrm{~m}$ tief. Weitere Daten unbekannt.

Die folgende Liste gründet sich auf die Beobachtungen IмноF's [1885] vom 26. VIII. 1884 und auf die Bestimmungen von Langhans [1911].

C o p e p o d a: Cyclops sp. [Імноғ].

Cl a d o c e ra: Alona affinis (Leydig) [Langhans]. Bosmina sp. [Імноғ]. Daphnia sp. [Імноғ]. Diaphanosoma brachyurum (Liévin) [Langhaxs]. Leptodora kindtii (Focke) [Ishof, Langhaxs]. Scapholeberis mucronata (O. F. MÜLler) [Langhans]. Sida crystallina (O. F. Müllek) [Langhans].

\section{Schwarzsee a. d. Turracherhöhe.}

(Bisher nicht veröffentlicht.)

Fundortsnummer: 144. Z one: Zentralalpen; Norische Alpen. Ge ographisehe Lage: An der Grenze von Kärnten und Steiermark auf der Paßhöhe von Turrach gelegen. S e eh öh e: ca. $1850 \mathrm{~m}$ ü. d. M. Fl u B g e bi e t: Predlitzgrabenbach-Nur-Drau. Gr ö ße: Ungefähr $250 \mathrm{~m}$ lang und $100 \mathrm{~m}$ breit. M a xi mal t i e fe: Nicht bekannt; nach den Angaben von Dr. K. HoLdHaus ein Seichtsee. 
Geologie der Umgebung: Liegt in der Kohlenformation. Waldgrenze.

In den von Herrn Dr. K. Holdhaus im Sommer des Jahres 1920 gesammelten Planktonnetzfängen wurden von uns folgende Arten nachgewiesen:

C o p e p o d a: Cyclops strenuus Fischer. Diaptomus gracilis $\mathrm{G}$. $\mathrm{O}$. SARS. Heterocope saliens LiLlJEBorg.

$\mathrm{Cl}$ a d o e e ra: Alona affinis (Leydig). Chydorus sphaericus O. F. Müller. Daphnia longispina var. hyalina Leydig. Peracantha truncata (O. F. MülLer).

\section{Schwarzensee im Zillertal.}

Fund ortsnum mer: 145. Z o n e: Zentralalpen; Zillertalergruppe der Hohen Tauern. G e o g r a p h is c h e L a g e: Tirol, Zemmgrund im Zillertal am Fuße des Schwarzensteingletschers bei der Berlinerhütte gelegen. S e e höh e: $2469 \mathrm{~m}$ ü. d. M. F lu Bg e bi e t: Zemmbach-Ziller-Inn-Donau. G r ö B e: Schmales Hochgebirgsbecken von ungefähr $300 \mathrm{~m}$ Länge. M a x i malt i e f e: Nicht ermittelt. G e o $10 \mathrm{~g}$ is ch e u ndh ydrographis ch e Dat e n: Nach Böнm [1886] ein echtes Felsbecken, durch Erosion entstanden. Kaltwas sers e e, auch im Hochsommer Eisschollen tragend.

Brehm und Zederbauer [1904] machten am. 20. VII. 1901 ergebnislose Fänge. Eine neuerliche Untersuchung, die Brenm [1906] am 20. VIII. 1904 ausführte, ergab das Vorkommen von:

Cyclops strenuus Fischer.

Schwarzer See beim Ödensee.

Fundortsnummer: 146. Z o ne: Nördliche Kalkalpen; Salzkammergutgebiet. Ge o gra ph is e h e La ge: Steiermark, Bezirk Aussee, südlich von Kainisch beim Ödensee.

Diesen Fundort erwähnt Langhans [1911] in seiner Bearbeitung der „Cladoceren aus dem Salzkammergut"; es fehlen jedoch nähere Angaben vollständig und es ist uns nicht gelungen, den See auf irgendeiner Karte ausfindig zu machen oder in einem geographischen Verzeichnis anzutreffen; es handelt sich vermutlich um ein sehr kleines Wasserbecken, dessen Benennung vielleicht nur den nächsten Ortskundigen geläufig ist. - Langhans [1911] gibt das Vorkommen von Alona affinis (Leydig) an. 


\section{Seebachsee.}

Fundortsnummer: 147. Z 0 n Klagenfurter Becken. Ge ograph is che Lage: Kärnten, am Südufer des Wörthersees beim Orte Secbach gelegen. Se ehöhe: $516 \mathrm{~m}$ ï. d. M. F l u ß g e b i e t: Secbach-Glanfurt-Drau. Gr ö Be: $1000 \mathrm{~m}$ lang, $210 \mathrm{~m}$ breit.

Імноғ [1890] hat als erster diesen Fundort untersucht; seine Angaben wurden später von SteuER [1897] im Verzeichnis aufgenommen. Demnach kommen hier folgende Gattungen bezw. Arten vor:

Co p e p o da: Cyclops sp.

Cla d o cera: Bosmina sp. Ceriodaphnia sp. Daphnia sp. Diaphanosoma brachyurum (LiÉvin). Monopsilus dispar G. O. SARS [ImhoF und Steuer sub M. tenuirostris]. Peracantha truncata (O. F. MüLler) [ІмноF und Steuer sub Pleuroxus tr.].

\section{Unterer Seebisee.}

Fu ndortsnummer: 148. Z 0 ne: Nördliche Kalkalpen; Allgäueralpen. G e $0 \mathrm{gr}$ a ph is e h e L a ge: Tirol, nordwestlich von Landeck im Gebiete der Parseierspitze gelegen. S e eh öh e: $2229 \mathrm{~m}$ ü. d. M. Flu ßg e b i e t: Seebibach-Parseierbach-AlperschonbachLech-Donau. Grö Be: 400 Schritte $(300 \mathrm{~m})$ lang; durchschnittlich 160 Schritte $(100 \mathrm{~m})$ breit. Nach unseren Beobachtungen [Pesta 1912] stellt der Untere Seebisee einen Flachsee von sehr geringer Tiefe dar; seine Ufer sind durchwegs flach, von moosbewachsenen Hügelwellen umgeben. Geologisehe und hydrographische Daten: Liegt in einer Liaskalkmulde. Beschaffenheit des Seebodens schlammig. Algenvegetation reich entwickelt. Am 15. VIII. 1911 wurde von uns eine Oberflächenwassertemperatur von $13,6^{\circ} \mathrm{C} \cdot$ gemessen. Che mis ehe Zus a m mensetzungdes Seewassers sieh e A bsehn. II (Tabelle).

Nach Pesta [1912] fanden sich im Unteren Seebisee folgende Arten:

Co pe poda: Cyclops serrulatus Fischer. Diaptomus bacillifer KOELBEL.

Cladoeera: Alona affinis (Leydig). Chydorus sphaericus O. F. MÜller. Macrothrix hirsuticornis Norman et Brady.

\section{Seraiasee (Lago di Seraia).}

Fun d o r ts nu mmer: 149. Z o n e: Südliche Kalkalpen; Südtiroler Dolomiten. Ge ogra ph is eh e Lage: Südtirol, nördlich 
vom Caldonazzosee im Val delle Pine. S e e höh e: $974 \mathrm{~m}$ ü. d. M. Fl u Bg e bi e t: Fersinabach-Etsch. Gr ö Be: $0,45 \mathrm{~km}^{2}$ Oberfläche; $1250 \mathrm{~m}$ lang, $525 \mathrm{~m}$ breit. Maxi maltiefe: $14,6 \mathrm{~m}$; im Mittel 6,5 m tief. Geologische und h ydrographische Daten: Liegt im Porphyr [Dalla Torre 1913]. Largaiolli [1898] beobachtete am 21. VIII. 1897 eine $\mathrm{O}$ berf $\mathrm{l}$ ä chenwass e r te m perat ur von 19,8 C; Buffa [1902] konstatierte im Monat August d. J. 1901 eine Temperatursteigerung des Oberflächenwassers von $16^{0}$ (um $6^{\mathbf{h}}$ morgens) auf $28,8^{0} \mathrm{C}$ (um $2^{\mathbf{h}}$ nachmittags). Von Mitte Dezember bis Ende März vereist. - F i s c h b e st a n d [nach Dalla Torre 1913]: Barbe, Hecht, Karpfen, Rotfeder, Schleie, Weißfisch u. a.

Wie aus der folgenden Aufzählung ersichtlich ist, weichen die Angaben über die Crustaceenfauna dieses Sees stark voneinander ab; die Liste gründet sich auf die Untersuchungen von LargaiolLi [1898] und Buffa [1902].

C o p e p o d a: Diaptomus castor Jurine [Largaiolit].

$\mathrm{Cl}$ a d o c e ra: Alona quadrangularis (O. F. MÜLler) [Buffa]. Bosmina coregoni-longispina Leydig [LaRgatolli sub $B$. longispina]. Bosmina longirostris (O. F. MÜLLER) [BUFFA]. Bosmina longirostriscornuta (JuRINe) [Buffa sub B. cornuta]. Daphnia longispina O. F. Müller [Largatolli]. Daphnia pulex (de Geer) [Largaiolli]. Sida crystallina (O. F. MülleR) [Largaiolit].

\section{Simssee.}

F u n d orts n u m mer: 150. Z o n e: Nördliche Kalkalpen. Voralpengebiet der Nordtiroler Kalkalpen. Ge ograph is che Lage: Bayern, östlich von Rosenheim. S e e höh e: $471 \mathrm{~m}$ ü. d. M. Flu B g e b i e t: Inn-Donau. Gr ö ß e: ca. $5 \mathrm{~km}$ lang, $1,5 \mathrm{~km}$ breit. M a ximaltiefe: $21 \mathrm{~m}$ [GeIstbeck 1885]. Ge ologische und hydrographische Daten: Liegt im Miocän. Sommerliche Oberfläch enwassertemperatur ca. $18^{\circ} \mathrm{C}$.

BRenм [1906] fand hier folgende Formen:

C o p e p od a: Cyclops sp. Cyclops macrurus G. O. Sars. Diaptomus gracitoides Lillueborg (nach einer Angabe von Baumbach [1922] ex BREHM [1909/10]).

Cl a d o c e r a: Bosmina coregoni var. acrocoregoni (BURCKHARDT) f. burckhardti. Daphnia longispina var. cucullata SARS [BREHM sub Hyalodaphnia c.]. Daphnia longispina var. hyalina LeYdig [BReHM sub D. hyalina]. Diaphanosoma brachyurum (LiÉvin). Leptodora 
kindtii (Focke) [BRehm sub L. hyalina]. Scapholeberis mucronata (O. F. MÜLLER).

\section{Sistransersee ${ }^{1}$ ).}

Fu nd ortsnummer: 151. Z o ne: Zentralalpen; Gebiet der Salzburger Schieferalpen. G e $0 \mathrm{~g} \mathrm{r}$ a $\mathrm{ph}$ i s e h e L a g e: Tirol, am südlichen Mittelgebirge von Innsbruck zwischen den Orten Aldrans und Sistrans gelegen. S e e h öh e: ca. $800 \mathrm{~m}$ ü. d. M. F l u Bg e b i e t: Herztalbach-Inn-Donau. Grö ße: Kleiner "See“ von etwa $100 \mathrm{~m}$ im Durchmesser; genaue Tiefenmaße nicht bekannt. Ge o l o g i e d e r U m g e bu n g: Phyllit.

ВREнм [1907] fand hier nur zwei Vertreter der Rotaterien, gibt jedoch keine Entomostraken an, die auch diesem Fundort nicht fehlen dürften; unter Hinweis auf die Notwendigkeit einer weiteren Untersuchung sei der See erwähnt.

\section{Sommersbergersee.}

Fun d ort s n u m mer: 152. Z o ne: Nördliche Kalkalpen; Salzkammergutgebiet. Ge ogr a phis che Lage: Steiermark, südwestlich von Aussee. S e e höh e: ca. $900 \mathrm{~m}$ ü. d. M. Flu Bg e bi et: Lupitschbach-Altausseertraun-Traun-Donau. Grö Be: Kleines, rundgeformtes Becken von etwa 250 Schritten im Durchmesser. Ti efe und die übrigen Daten nicht bekannt.

Langhans [1911] bestimmte aus den ihm vorliegenden Proben folgende Cladoceren:

Acroperus harpae BAIRD. Alona affinis (LEYDIG). Alona costata G. O. Sars. Alona guttata G. O. SARS. Alona quadrangularis (O. F. Müller). Bosmina longirostris (O.F. MüLler) [LANGHans sub B. mülleri!]. Bosmina longirostris-cornuta JuRINe. Ceriodaphnia pulchella G. O. SARS. Simocephalus serrulatus (Косн).

\section{Oberer Sonntagskarsee.}

\section{(Bisher nicht veröffentlicht.)}

Fund o r t s n u m mer: 153. Z on e: Zentralalpen; Niedere Tauern. Ge og r a p h i s e h e L a g e: Steiermark, südlich von Schlad-

1) Der Name „Sistranser-See“ ist unseres Wissens in der Gegend nicht gebräuchlich, obwohl ihn auch Dalla TORRE [1913] aufgenommen hat. Auf der im Jahre 1921 vom Deutschen und Österreichischen Alpenverein herausgegebenen Karte des Brennergebietes findet sich keine derartig benannte Lokalität. Es handelt sich zweifellos um den sogenannten „Herzsee“ (halbwegs zwischen Aldrans und Sistrans), welchen Dalla Torre [1913] n i c h t erwähnt; dieser See ist auf allen Karten angegeben und auch im Katasterverzeichnis der Tiroler Seen enthalten. 
ming am FuBe der Waldhornspitze $(2700 \mathrm{~m})$ gelegen. S e eh öh $\mathrm{f}$ : 2036 m ii. d. M. F l u B g e b i e t: Rissach bach —Talbach-Enns-Donau. G r ößc: Der See mißt im Durchmesser ungefähr 300 Schritte. Ti e fe nicht ermittelt, jedoch handelt es sich zweifellos um ein tieferes Trichterbecken. Geologische und hydrographisehe Daten: Liegt im Glimmerschiefer. Nach Böнм [1886] ein echtes Felsbecken, durch Erosion entstanden. Uferumrandurg vom Südwesten bis Südosten durchwegs aus Steilabstürzen (zum Teil Felswänden) bestehend, nur im Norden (am Seeabfluß) frei. S e e b od e n nirgends versumpft, ausgesprochen glimmerig-sandig. Zuflüsse zum Teil mächtigere Wasserfälle. Vereiste Schneemassen und schwimmende Eisblöcke am 17. VIII. 1919 beobachtet. Makroskopisch wahrnehmbare W a s s e r f l o r a vollständig fehlend; nur in den Planktonnetzproben gr üne Schwєbealgen nachweisbar. Wasser klar, den Seeboden bis auf 10-15 m Entfernung vom Ufer noch deutlich sichtbar lassend, von dunkelgrüner Färbung. Es wurde von uns am schon erwähnten Datum um $9^{\text {h }} 45^{\prime}$ a. m. eine $\mathrm{Ob}$ e r f 1 ä c h e nwas s e r e m per a t u r von $3,2^{\circ} \mathrm{C}$ gemessen (bei gleichzeitiger Luit. temperatur von $12,3^{\circ} \mathrm{C}$ im Schatten); die Wassertemperatur von zwei Zuflüssen betrug $7^{\circ} \mathrm{C}$ bezw. nur $2,5^{0} \mathrm{C}$. C h e $\mathrm{m}$ i s e h e $\mathrm{Z}$ u s a m m e rsetzung des Wassers siehe Abschnitt. I und IF (Tabelle).

Es wurden in den von uns gesammelten Planktonnetzfängen folgende Formen gefunden:

C o p e p o da: Canthocamptus cuspidatus SchmeIL.

Cl a d o c e ra: Bosmina sp. Acroperus harpae Barid.

\section{Unterer Sonntagskarsee.}

(Bisher nicht veröffentlicht.)

Fundortsnummer: 154. Z one: Zentralalpen; Niedere Tauern. Ge o gr a ph is e h e L a ge: Wie vorhin bei Nr. 153. S e e h öh e: ca. 1930 m ü. d. M. Flu ßg e b i e t: Wie bei Nr. 153. Gr öß e: Ungefähr 300 Schritte im Durchmesser. T i e f e nicht ermittelt; nach der Gestaltung der Umrandung zweifellos die Tiefe des Oberen Sonntagskarsees nicht erreichend, jedoch ebenfalls kein Seichtsee. Ge $0 \mathrm{log}$ i s $\mathrm{ch} \mathrm{e}$ u n d h y drogra ph is c h e D a t e n: Liegt im Glimmerschiefer und gehört nach Böнм [1886] gleichfalls zu den durch Erosion entstandenen, echten Felswannen. Ufer mit. Ausnahme einer kleinen Geröllhalde im Westen und der Felsabbrüche der an der Südseite angrenzenden Steilwandstufe aus begrasten, niederen Hängen bestehend, insbesondere im Bogen von Nordwesten über Norden nach Nordosten flach. S e e b o d e n 
steinig und sandig, an keiner Stelle sumpfig. W a s s e r f I o r a gering entwickelt, Algen beobachtet. Die zahlreich am Seegrund wahrnehmbaren Moosballen gelangen offenbar mit den abrollenden Steinen in das Becken. Färbung des Wasserspiegels dunkelgi ün. Die O b e r flächenwassertemperatur betrug am 17. VIII. 1919, wo Ufer und See vollkommen schnee- und eisfrei waren, an drei verschiedenen Stellen 7,50 C (in nächster Nähe des größten Zuflusses, Wasserfall) bezw. $8^{\circ} \mathrm{C}$ und $9^{\circ} \mathrm{C}$; die Temperatur des Hauptzuflusses maß gleichzeitig $6^{\circ} \mathrm{C}$. Zahlreiche Fische (? Phoxinus), die dem oberen Sonntagskarsee zu fehlen scheinen, wurden von uns beobachtet. - Che mis ehe $\mathrm{Zu}$ sammensetzung des Wassers siehe Absehnitt I u n d II (Tabelle).

In den Planktonnetzfängen wurden von uns folgende Entomostraken nachgewiesen:

C o p e p o d a: Canthocamptus cuspidatus SchmeIL. Cyclops serrulatus Fischer. Cyclops vernalis Fischer.

$\mathrm{Cl}$ a d o c e ra: Acroperus harpae Baind. Alona affinis (Leydig) var. ornata Sting. Alona quadrangularis (O. F. MüLler).

\section{Sonthofersee ( $=$ Niedersonthofersee $)$.}

Fundortsnu mmer: 155. Z o ne: Nördliche Kalkalpen; Allgäucr Alpen. Ge ographis ch e Lage: Südbayern, nördlich von Immenstadt an der Mler. S e e h ö h e: ca. $700 \mathrm{~m}$ ü. d. M. F l u B g e b i e t: Iller-Donau. Gr öße: Über $2,5 \mathrm{~km}$ lang, $0,525 \mathrm{~km}$ breit. Ti e fe: Unbekannt (?).

Імноғ [1887] determinierte aus seinen Planktonfängen vom August bis Septerber folgende Genera bezw. Spezies:

Co p e p o da: Cyclops sp. Diaptomus sp.

C l a d o c e ra: Daphnia sp. Diaphanosoma brachyurum (LiÉvin). Leptodora kindtii (FockE).

\section{Speiksee.}

Fund ortsnummer: 156. Z o ne: Zentralalpen; Norische Alpen. Ge ogra ph ische Lage: Steiermark, nächst del Grenze von Kärnten, östlich von Wolfsberg im Gebiete der Koralpe. Se e h öh e: 1822 m ü. d. M. Flu ßg e bi et: Seebach-Schwarze SulmSulm-Mur-Drau. Größ e: ca. $100 \mathrm{~m}$ im größten Durchmesser. Ge $0 l o g$ isehe und hydrographisehe Daten: Nach den Angaben von Dr. K. Holdhaus besteht die Umgebung des Speiksees 
aus kristallinen Schiefern; er wird als sehr seicht bezeichnet, sein Boden als steinig und schlammig; bildet eine Viehtränke.

Pesta [1911] meldet das Vorkommen von zwei C o p e p o de n s pezi es, nämlich:

Cyclops vernalis Fischer. Diaptomus denticornis WierzeJSKI.

\section{Spitzingsee.}

Fu n d o r t s n u m m e r: 157. Z o n e: Nördliche Kalkalpen; Nordtiroler Kalkalpen. Ge o gra ph is che Lage: Südbayern, südlich vom Schliersee (Nr. 140). S e e h ö h e: 1075 m (1082 m) ü. d. M. Fl u ßg e bi e t: Rote Valepp-Brandenbergerache-Inn-Donau. Gr ö B e: ca. $900 \mathrm{~m}$ lang, größte Breite ca. $450 \mathrm{~m}$. T i e f e: 15 m [GeIst becK 1885]. Ge ologische Dat e n: Nach Böнm [1886] ein echtes Felsbecken, entstanden durch Einbruch oder Auflösung des Kalkes.

Імноғ [1887] fand in seinen aus den Monaten August und September stammenden Planktonfängen folgende Formen:

C o p e p o d a: Cyclops sp. Diaptomus sp.

C l a d o c e r a: Bosmina sp. Daphnia sp. Leptodora kindtii (Focke). Scapholeberis mucronata (O. F. MüLLER). Sida crystallina (O. F. MüLLER).

\section{Staffelsee.}

Fu nd orts n u m mer: 158. Z o n e: Nördliche Kalkalpen; Ammergauer Alpen des Nordtiroler Kalkalpenzuges. G e o g r a ph i s ch e L a g e: Südbayern, bei Murnau südlich vom Starnbergersee. S e e h ö h e: $601 \mathrm{~m}$ (649 m!) ü. d. M. F l u ß g e b i e t: Ach-Ammer-IsarDonau. Grö ß e: Großer Alpenrandsee von ungefähr $3,5 \mathrm{~km}$ Durchmesser, reich gegliedert und mit einigen Inseln. M a x i mal $\mathrm{t}$ i e f e: $34 \mathrm{~m}$. Geologische und hydrographische Daten: Miocän; nach Wallmann [1868] im Nagelfluhgestein. Geistbeck [1885] maß am 9. VIII. 1881 eine $\mathrm{O}$ ber f 1 ä chen was serte mperatur von $22,2^{\circ} \mathrm{C}$.

Auch an diesem Fundort sammelte IмноF [1887] Plankton (August bis September) und gibt folgende Genera und Spezies an:

Co pe poda: Cyclops sp. Diaptomus sp.

C I a d o c e r a: Bosmina sp. Daphnia sp. Diaphanosoma brachyurum (LIÉviv). Leptodora kindtii (FocKE).

\section{Starnbergersee (Würmsee).}

F u n d orts nu mmer: 159. Z o ne: Voralpenbereich der Nördlichen Kalkalpen; Nordtiroler Kalkalpen. Ge o g r a ph is c h e L a g e: 
Südbayern, südwestlich von München. S e e h öh e: $584 \mathrm{~m}$ ü. d. M. Flu Bge bi et: Würm-Isar-Donau. Grö B e: Großer Voralpensee von $21 \mathrm{~km}$ Länge und einer Breite bis zu $5 \mathrm{~km} ; 57 \mathrm{~km}^{2}$ Oberfläche. Maximaltiefe: $115-120 \mathrm{~m}$. Ge ol o gische und hydro$\mathrm{graph}$ is eh e Daten: Nach der Auffassung von Penk [Halbfass 1913] ist der Starnbergersee durch Gletschererosion während der letzten Vereisung entstanden, nach jener von ULE ein durch fließendes Wasser gebildeter Erosionssee, der als interglaziales Talsystem schon vor der letzten großen Vergletscherung zustande kam. O ber $\mathrm{flä} \mathrm{che} \mathrm{n-}$ wassertemperatur am 12. IX. 1881 nach Geistbeck [1885] $15,7^{\circ}$ C. - Enthält Fische, davon bes onders bekannt seine Renken.

Die folgende Liste ist den Ergebnissen der Arbeiten von HofER [1896], Zacharias [1905] und Brehm [1907] entnommen; demnach kommen vor:

Co p e po da: Cyclops sp. [Zacharias]. Cyclops strenuus Fischer [Brehm, Zacharias]. Diaptomus gracilis G. O. Sars [Hofer, Zacharias sub D. graciloides, BREHм corr. in D. gracilis]. Heterocope weismanni IMHoF [HoFer sub $H$. robusta; $H$. robusta kann sich sowohl auf $H$. weismanni als auch auf $H$. saliens LiLLJ. beziehen; nach den Angaben vaN Douwe's [1909] muß im vorliegenden Falle eine Identifizierung mit der ersteren Spezies vorgenommen werden].

$\mathrm{Cl}$ a d o e e r a: Bosmina sp. [BReHM]. ? Bosmina coregoni-longispina Leydig f. bohemica Sting. [Zacharias sub Bosmina ? bohemica]. Chydorus sphaericus O. F. Müller [Zacharias]. Diaphanosoma brachyurum (Lívin) [Zacharias].

\section{Stellunesee (Lago delle Stellune).}

Fu nd orts nu m mer: 160. Z o n e: Südliche Kalkalpen; Südtiroler Dolomiten. Ge ogr a ph is ch e Lage: Südtirol, südlich von Cavalese im Fassatal, am Nordfuße der Montalonkette. Se eh öh e: $2140 \mathrm{~m}$ ü. d. M. F l u ßg e b i e t: Riva delle Stue-Riva di CadinoAvisio-Etseh. Größe: $225 \mathrm{~m}$ lang, $180 \mathrm{~m}$ breit. Maximaltiefe: 18,2 m.

Buffa [1902], der diesen Hochgebirgssee am 29. VIII. 1900 untersuchte, meldet lediglich das Vorkommen von Canthocamptus minutus Claus.

\section{Tegernsee.}

F u n d or ts n u m mer: 161. Z o n e: Nördliche Kalkalpen; Nordtiroler Kalkalpen. G e o gr a p h i s c h e L a g e: Südbayern, südwestlich 
von Miesbach. S e e h öh e: $726 \mathrm{~m}$ ü. d. M. F l u Bg e b i e t: WeißachMangfall-Inn-Donau. Gr ö Be: $6 \mathrm{~km}$ lang, $2 \mathrm{~km}$ breit. M a $\mathbf{x}$ i m a ltiefe: $96 \mathrm{~m}$. Geologisehe und hydrographisehe Dat e n: Moränensee. Nach Halbfass [1913] sind die Unebenheiten in seinem Bodenrelief vielleicht durch die verschiedene Geschwindigkeit beim Rückzuge der Moränen erklärbar. GEISTbECK [1885] maß am 4. IX. 1881 eine Oberflächenwassertemperatur von $14,4^{\circ} \mathrm{C}$.

Über die Planktoncrustaceen des Tegernsees haben Iмноғ [1887] und Richard [1896] Angaben geliefert; es wurde von ihnen das Vorkommen folgender Gattungen und Arten nachgewiesen:

Co pe poda: Cyclops sp. [Імноғ]. Cyclops strenus Fischer var. [Richard]. Diaptomus sp. [Imhof]. Diaptomus gracilis G. O. SARS [RICHARD].

Clad ocera: Bosmina sp. [Iмноғ]. Bythotrephes longimanus Lexdig [Richard]. Daphnia sp. [Iмнof]. Daphnia longispina var. hyalina Leydig [RICHARd sub D. hyalina]. Leptodora kindtii (Focke) [ImhoF, Richard].

\section{Terlagosee (Lago di Terlago).}

F u n d o rts n u m mer: 162. Z o n e: Südliche Kalkalpen; Etschbuchtgebirge. C e v g r a ph is ch e L a g e: Südtirol, nordwestlich von Trient, am rechten Etschufer. Seehöh e: $416 \mathrm{~m}$ ü. d. M. Flu Bg e bi e t: Riva di Vela-Etsch (Adria). Gr ö B e: 0,29 km² Oberfläche; $1450 \mathrm{~m}$ lang, $300 \mathrm{~m}$ breit (100 m geringste Breite). M a x i m a l t i e f e: 9,2 m. Geologisehe und hydrographisehe Daten: Liegt im Gebiet der Liaskalke. Nach DalLa ToRre [1913] ist der See in raschem Verschwinden begriffen; zerfällt durch eine $75 \mathrm{~m}$ breite und $2 \mathrm{~m}$ unter dem Wasserspiegel befindliche Bodenwelle in zwei Teile. Wasser wenig durchsichtig, von braungrüner bis olivgrüner Färbung. Largaiolli [1906] maß die O b e r f lä ch e n wa s s e r te mperatur am 3. VIII. 1904 mit $24^{\circ} \mathrm{C}$, am 25. VIII. 1905 mit $19,5^{0} \mathrm{C}$.

Largatolli [1906] fand folgende Formen:

C o p e p o d a : Cyclops strenuus Fischer.

$\mathrm{Cl}$ a d o e e ra: Alonella nana (BAird) [Largaiolli sub Pleuroxus. nanus]. Bosmina longirostris-cornuta JURINE. Diaphanosoma brachyurum (LiÉvin). Diaphanosoma brachyurum var. tridentinum LARG. Leptodora kindtii (Focke). Rhynchotalona falcata (SARS) [Largaiolli sub Alona $\%$.]. Sida crystallina (O. F. MülleR). 


\section{Thumsee.}

F u n d o r t s n u m mer: 163. Z o n e: Nördliche Kalkalpen; Berchtesgadnergruppe der Salzburger Kalkalpen. G e 0 g r a p h i s e h e L a g e: Südbayern, westlich von Reichenhall. S e e höh e: $527 \mathrm{~m}$ ü. d. M. Flu Bg e bi et: Saalach-Salzach-Inn-Donau. Grö Be: $900 \mathrm{~m}$ lang, größte Breite $300 \mathrm{~m}$.

Brenm [1906], welcher an diesem Fundort am 16. VIII. 1904 Plankton aufsammelte, meldet das Vorkommen folgender Spezies:

C o p e p o d a: Diaptomus gracilis G. O. SArs.

$\mathrm{Cl}$ a d o e e r a: Bosmina longirostris (O. F. MülLer). Ceriodaphnia quadrangula (O. F. MüLLER).

\section{Tiersee.}

F u n d o r t s n u m me r: 164. Z o n e: Nördliche Kalkalpen; Nordtiroler Kalkalpen. Ge $0 \mathrm{graph}$ is che $\mathrm{Lage:}$ Nordtirol, westlich ron Kufstein nahe der bayerischen Grenze. S e e h ö h e: $616 \mathrm{~m}$ ï. d. M. Flu Bg e biet: Klausenbach-Tierseeache-Inn-Donau. Größe: ca. $750 \mathrm{~m}$ lang und ca. $400 \mathrm{~m}$ breit; 25,7 ha Oberf:äche. M a x i m a ltiefe: $13,2 \mathrm{~m}$. Geologisehe und hydrographisehe D a $\mathrm{t}$ e n: Müllner [1905] bezeichnet das Seebecken als eine aus Mergeln herausgearbeitete Wanne; am Ostufer Urgesteinsgeschiebe glazialen Ursprunges, daneben Reste von Alttertiär. Bes eh affenheit des S e e gr u n d e s: Schlammig. - Enthält nach Dalla Torre [1913] folgende Fische: Aitel, Barsch, Hecht, Pfrille, Rotfeder, Schleie, Schmerle und Steinpitzger.

In den vom 25. VIII. stammenden Plarktonfängen wies BREHM [1907] folgende C l a d o c e r e n nach:

Bosmina longirostris (0. F. MüLleR). Ceriodaphnia quadrangula var. hamata LILLJEBORG.

\section{Tihojasee.}

F u n d orts n u m mer: 165. Z o ne: Vermutlich Südliche Kalkalpen (? Klagenfurter Becken). G e o g r a ph i s c h e L a g e: Kärnten. - Alle anderen Daten unbekannt, da es nicht gelang, diesen Fundort auf irgendeiner Karte oder in einem geographischen Verzeichnis aufzufinden. Імноғ [1890] zählt diesen See auf; seine darauf bezüglichen faunistischen Angaben wurden später von SteuER [1897] übernommen; demnach kommen hier vor:

Co pe po da: Cyclops sp. 
Cl a d o c e r a: Acroperus harpae Baird [ImHof sub A. leucocephalus]. Alona rectangula SARS [Імноғ sub A. lineata]. Peracantha truncata (O. F. Müller) [ImhoF sib Pleuroxus tr.].

\section{Tilisunasee.}

F u n d o r t s n u m m e r: 166. Z o n e: Nördliche Kalkalpen; Rhätikongebiet der Allgäuer Alpen. Ge ograph is che Lage: Vorarlberg, südwestlich von Schruns im Montafon. S e e h ö h e: $2102 \mathrm{~m}$ ü. d. M. Flu Bge biet: Ill-Rhein. Grö Be: Úber $300 \mathrm{~m}$ lang und $150 \mathrm{~m}$ breit. Maximaltiefe: ca. 10-15 m. Ge ologische und h y drograph is ch e D a t e n: Seebecken am Nordfuße der Sulzfluh (Kreide), teils in Kalkgesteinen, teils in Gneis und Glimmerschiefern gelegen. Zsснокке [1890] gibt für die Monate Juli und August eine Oberflä chenwasserte mperaturvon $10-15^{\circ} \mathrm{Can}$. Fis chbe st and [nach Dalla Torre 1913]: Pfrille, Koppe.

Zsснокке [1890 und 1900] fand im Tilisunasee folgende Spezies:

Co p e p oda: Cyclops strenuus Fischer. Canthocamptus rhaeticus Schmeil. Cyclops vernalis Fischer. Cyclops serrulatus Fischer. Diaptomus bacillifer KOELBEL.

Cla d o e era: Chydorus sphaericus O. F. Müller. Daphnia longispina O. F. MÜLLER. Leydigia acanthocercö̈des (FisCHER) [ZSCHOKKE sub Lynceus a.]. Macrothrix laticornis (JURINE). Rhynchotalona rostrata (Косн) [ZschокKe sub Alona quadrangularis corr. in A. rostratus].

\section{Toblachersee.}

Fu n d ortsnum mer: 167. Z o n e: Südliche Kalkalpen; Südtiroler Dolomiten. Ge o gra ph is ch e Lage: Südtirol, südlich von Toblach im Pustertale. S e e h öh e: $1259 \mathrm{~m}$ ü. d. M. F l u B g e bi et: Rienz-Eisack-Etsch. Gr ö ß e: ca. $800 \mathrm{~m}$ lang und $400 \mathrm{~m}$ breit. M a ximalt i e fe: Nicht bekannt, angeblich seicht. Ge o l o g i s e h e und hydrographische Daten: Liegt im Dolomitgestein; Stausee durch Schuttkegel [Dalla Torre 1913]. - Enthält Fo r elle n.

Auffälligerweise enthielt der See nach den Untersuchungen von Brehm und Zederbauer [1905], welche hier am 3. VIII. 1903 fischten, „außer einigen Fäden von Zygnema (Algen) kein Plankton“. Zweifellos beruht dieses Ergebnis auf einem bedauerlichen Irrtum, der eine Nachuntersuchung dringend verlangt. 


\section{Toblinosee (Lago di Toblino).}

F u nd orts n u m mer: 168. Z o n e: Südliche Kalkalpen; Etschbuchtgebirge. Ge ographische Lage: Südtirol, westlich von Trient, am Knie der Sarca gelegen. S e e h öh e: $250 \mathrm{~m}$ ü. d. M. Flu B $\mathrm{g}$ e b i e t: Rimonebach-Sarca-Mincio-Po (Adria). G r ö ße: $1600 \mathrm{~m}$ lang, größte Breite $800 \mathrm{~m}$. M a x i mal t i e fe: $40 \mathrm{~m}$. Ge o lo g i s ch e u nd h ydrograph is che Daten: Liegt in Eocänmergeln; nach Dalla Torre [1913] ein durch Bergsturz entstandener Stausee. Reichgegliedertes Becken mit vielen Halbinseln und tiefen Buchten. W a s s e r fär bu ng dunkel. En thält a n F is ch en: Aal, Barbe. Forelle, Hecht, Karpfen, Koppe, Rotfeder, Schleie und andere.

Über die Cladoceren und Copepoden des Toblinosees haben HeLler [1871], Pavesi [1882] und Largaiolli [1898] berichtet und geben folgende Spezies an:

Co p e p o da: Cyclops fuscus Jurine [Heller sub C. coronatus; Pavesi sub C. coronatus; Largaiolli]. Cyclops serrulatus Fischer [Pavesi, Largatolli].

Cla d o c e ra: Bosmina longirostris (O. F. Müller) [Pavesi, Largaiolli]. Ceriodaphnia quadrangula (O. F. Müller) [Largaiolit].

\section{Todtalpsee.}

F u n d o r t s n u m mer: 169. Z o n e: Nördliche Kalkalpen; Rhätikongebiet der Allgäuer Alpen. G e $0 \mathrm{~g} \mathrm{r}$ a p h i s c h e L a g e: Vorarlberg; am Ostabhange der Scesaplana (Todtenalp). S e e h ö h e: $2340 \mathrm{~m}$ ü. d. M. Flu ßgebiet: Ill-Rhein. Größe: $200 \mathrm{~m}$ lang. Maximalt i e f e: Wenige Meter. Ge o log is che undh y d o graph is che D a t e n: Liegt im Kalkgestein. Nach Zschoкke [1894] ein Schmelzwassersee; der genannte Autor fand ihn am 24. VII. 1891 zugefroren, am 24. VIII. 1893 offen mit einer 0 bec $\mathrm{fl} l a ̈$ ch en was ser temperatur von $6^{\circ} \mathrm{C}$.

ZschоккE [1894] fand in diesem Seebecken wohl sechs verschiedene Tierspezies, konnte jedoch ein Vorkommen von Copepoden und Cladoceren nicht nachweisen.

\section{Toplitzsee.}

Fundorts nu m mer: 170. Z o n e: Nördliche Kalkalpen; Salzkammergut. Geographische Lage: Steiermark, östlich rom Grundlsee (Nr. 45). S e e h öh e: $716 \mathrm{~m}$ ü. d. M. Flu Bg ebiet: Gößlbach-Traun-Donau. Gr ö ß e: $1,9 \mathrm{~km}$ lang und ca. $400 \mathrm{~m}$ breit; 
0,54 $\mathrm{kn}^{2}{ }^{2}$ Oberfläche. M a x i maltiefe: $106,2 \mathrm{~m}$, im Mittel $62,4 \mathrm{~m}$ ticf. Ge ologische und h y drographis che Daten: Nähere Angaben nicht bekannt. Ufer steilgeneigte, bewaldete Hänge.

Langhans [1911] gibt für diesen Fundort zwei Cla d o c e r e n an, nämlich :

Bythotrephes longimanus (LEYDIG). Diaphanosoma brachyurum (LIÉvin).

\section{Unterer Torjochsee.}

(Bisher nicht veröffentlicht.)

Fundortsnummer: 171. Z one: Zentralalpen; Tuxer Tonschiefergebiet der Salzburger Kalkalpen. Ge o gr a p h i s c h e L a g e: Tirol; am Torjoch, dem Übergang vom Wattental in das Tuxertal (Zillertal) gelegen. S e e h ö h e: ca. $2200 \mathrm{~m}$ ü. d. M. F l u B g e b i et: Torseebach-Tuxerbach-Ziller-Inn-Donau. Gr ö B e: ca. $150 \mathrm{~m}$ lang und $100 \mathrm{~m}$ breit. M a x i malt i e f e: Nicht ermittelt, der Gestaltung nach ein Trichtersee mit größerer Tiefe. Ge ologische und hydrographische Daten: Liegt im Kontaktgebiet von Schiefern und Kalkgesteinen. Seeufer von niedrigen, grasbewachsenen Alpenriegeln gebildet, an der Südwestseite beim Zufluß aus dem oberen Torjochsee etwas verschilft und seicht, Sandbankbildung; hier auch zahlreiche Algen entwickelt. B o d e $\mathrm{n} \mathrm{b}$ e $\mathrm{s} \mathrm{ch}$ a f f e $\mathrm{nh}$ e i t im allgemeinen schlammig. Am 9. VIII. 1921 um $9-11^{\mathbf{h}}$ a. m. wurde von uns eine $0 \mathrm{ber}$ fläehenwassertemperatur von $14,3^{0}$ C festgestellt; die Temperatur des Zuflusses aus dem oberen Torjochsee betrug $12,8^{\circ} \mathrm{C}$, jene eines als Quelle am Südwestufer entspringenden zweiten Rinnsales nur $7,3^{\circ}$ C. Wasserfärbung: Grün, klar und durchsichtig. Fische nicht beobachtet.

In den am 9. VIII. 1921 von uns gesammelten Planktonnetzfängen fanden sich folgende Arten:

Copepoda: Cyclops strenuus Fischer. Diaptomus bacillifer KOELBEL.

Cla d o c e ra: Chydorus sphaericus O. F. Müller. Daphnia longispina var. longispina s. str.

\section{Tovelsee (Lago di Tovel).}

F u n d o r t s n u m mer: 172. Z o n e: Südliche Kalkalpen; Nonsbergergruppe des Etschbuchtgebirges. Ge ographische Lage: Südtirol; südlich von Cles (Nonsberg). S e e h öh e: $1162 \mathrm{~m}$ ü. d. M. Flu Bg e bi e t: Terresengabach-Noce-Etsch (Adria). Größe: 
$0,52 \mathrm{~km}^{2}$ Oberfläche; fast $1000 \mathrm{~m}$ lang, $300 \mathrm{~m}$ breit. M a $\mathrm{x}$ i $\mathrm{m}$ a $\mathrm{l}$ t i e f $\mathrm{c}$ : $35 \mathrm{~m}$. Geologische und hydrographische Daten: Liegt im Dolomit. Friert im Winter tragfähig zu [DALla TorRe 1913]. LARGıiolli [1907] maß während der Sommermonate der Jahre 190:2 bis 1906 oberflächliche $\mathrm{W}$ a s s e r t e m p e r a t u r e n von $17-19,4^{0} \mathrm{C}$. - F i s ch be st a $\mathrm{nd}$ : Pfrille und Saibling.

Largatolli [1907] fand folgende Spezies:

Co p e p o d a: Cyclops diaphanus Fischer [Larg. sub C. minutus]. Cyclops strenuus Fischer.

Cladocera: Alona affinis (LEYDIG). Chydorus sphaericus O. F. Müller (Type B Stingelin). Daphnia longispina var. hyalina LEYDig [LARG. sub D. hyalina].

\section{Traunsee (Gmundenersee).}

F u n d o r t s n u m mer: 173. Z o n e: Nördliche Kalkalpen; Salzkammergutgebiet. Ge o gra ph is ch e L a g e: Oberösterreich, bei Gmunden. S e e h ö h e: $422 \mathrm{~m}$ ü. d. M. F l u B g e b i e t: Traun-Donau. Gr ö B e: $25,65 \mathrm{~km}^{2}$ Oberfläche; $12 \mathrm{~km}$ lang, $3 \mathrm{~km}$ breit. M a x i m a I t i e f e: $191 \mathrm{~m}$, im Mittel 89,75 m tief. Ge o lo gis che und h y d r ogra phis che Date n: Nach der Auffassung von Penk [Halbfass 1913] ging der Querbruch des umgebenden Gebirges der Entstehung des Seebeckens, welches sich erst während der Eiszeitperioden bildete, roraus. Liegt in Liaskalken und (nördlich) in Molasse [WaLlmann 1868]. O b e rfl ä ch e n w a s s e r t e m p e r a t u r während der Dauer eines Jahres (1898/99) im Minimum 4,90 C und im Maximum 17,30; Jahresmitteltemperatur des gesamten Seewassers rund $5^{\circ} \mathrm{C}$ [vergl. dazu Langhans 1908]. Vereisung sehr selten (Februar bis Mitte Mai). Auffallenderweise sind die Wassertemperaturen an seichten Uferstellen oft weit niederer als über großen Tiefen (z. B. $13,8^{\circ} \mathrm{C}$ am Ufer, $20,8^{\circ} \mathrm{C}$ über einer Tiefe von $88 \mathrm{~m})$. Phyto pla $\mathrm{kt} 0 \mathrm{ngeh}$ a $\mathrm{lt}$ des Sees [rach Langhans 1908] reich (26 pelagische Genera). Wasserfär b u n g: Dunkelgrün. - Enthält folgende Fisch e: Alburnus lucidus, Coregonus exiguus, C. wartmanni, Cottus gobio, Esox lucius, Leuciscus rutilus, Lota vulgaris, Perca fluviatilis, Scardinius erythrophthalmus, Trutta lacustris.

Die folgende Liste gründet sich auf die Untersuchungen von CLAUs [1877], Imhof [1885], Cori [1898], Brehm und Zederbauer [1906]. Keissler [1907], Langhans [1908] und Langhans [1911]. Es wurden bisher folgende Formen festgestellt: 
Co p e p oda: Cyclops sp. [Imhof, Keissler]. Cyclops leuckarti Claus [Cori, Langhans 1908]. Cyclops serrulatus Fischer [Brehm und Zederbauer, Langhans 1908]. Cyclops strenuus Fischer [Cori, Brehm und Zederbauer, Langhans 1908]. Diaptomus sp. [Imhof, Keissler]. Diaptomus gracilis G. O. Sars [Cori, Brehm und Zederbauer, Langhans 1908]. Heterocope saliens Lilljeborg [Імнof sub H. robusta; bezieht sich nach Langhans 1908 auf H. saliens!].

Cladocera: Acroperus harpae Baird [Langhans 1908 sub A. leucocephalus]. Alona affinis (Leydig) [Langhans 1908]. Alona costata G. O. Sars [Langhans 1908]. Alona guttata G. O. Sars [Cori]. Bosmina coregoni Batrd [Brehm und Zederbauer]. Bosmina coregonilongispina Leydig [Langhans 1908 und 1911 sub B. longispina!]. Bosmina longirostris O. F. MÜller [ClaUs, CoRI]. Bosmina longirostriscornuta Jurine [Cori, Langhans 1908 sub B. cornuta!]. Bythotrephes longimanus Leydig [Claus, Cori, Brehm und Zederbauer, Langhans 1908 und 1911]. Camptocercus rectirostris (SchoedLer) [CoRI]. Daphnia longispina O. F. MüLLER [CORI]. Daphnia longispina var. hyalina LEYdiG [Claus, Cori, Brehm und Zederbauer, Langhans 1908 und 1911] - alle Autoren sub D. hyalina -. Diaphanosoma brachyurum (LiÉvis) [ImhoF, Langhans 1908]. Eurycercus lamellatus (O. F. Müller) [Langhans 1908 und 1911]. Leptodora kindtii (Focke) [Claus, Cori, Brehm und Zederbauer, Keissler, Langhans 1908 und 1911]. Peracantha truncata (O. F. Müller) [Langhans 1908 sub Pleuroxus tr.]. Pleuroxus sp. [Langhans 1908]. Polyphemus pediculus (Linné) [Claus, Cori, LangHANs 1908 und 1911]. Rhynchotalona rostrata (КосH) [CoRI sub Alona r.]. Sida crystallina (O. F. Müller) [Claus, Langhans 1908].

Bezüglich der in dieser Liste verzeichneten Spezies sei besonders auf die merkwürdige Tatsache hingewiesen, daß Імноғ's [1885] Angabe über das Vorkommen von Heterocope im Traunsee seither keine Bestätigung mehr fand.

\section{Tschampeisee (Tschampatschsee).}

Fu nd orts n u m mer: 174. Z o n e: Südliche Kalkalpen; Südtiroler Dolomiten. G e $0 \mathrm{gr}$ a $\mathrm{ph}$ i s c h e L a g e: Südtirol, nächst dem Tschampeijoch nördlich von Collfuschg (Enneberg) am Fuße des Gardenazzastockes. S e e h öh e: ca. $2100 \mathrm{~m}$ ü. d. M. Flu B g e bi e t: Piscadoibach-Gaderbach-Rienz-Eisack-Etsch. Grö Be: 140 Schritte lang, 80 Schritte breit. Ma ximalti efe: Ein Seichtsee von kaum mehr als einem halben Meter im Maximum. Ge ologis che und 
hydrographisehe Daten: Liegt in Dachsteindolomit. Ufer durchwegs sehr flach, gerölfführend, am Ostrande etwas sumpfig (Ufertiefen höchstens $10-15 \mathrm{~cm})$. Boden beseh affenheit weich, stark schlammig. F är bu ng des Wassers lehmgelb. Makroskopische W a s serflor a sowie auch Phytoplankton nicht nachgewiesen. Als (Oberflächen-) Wassertemperaturen wurden von uns am 12. VII. 1913 mittags $9,6 \check{D}-9,7^{\circ} \mathrm{C}$ gemessen; die Temperatur des von Norden über Fels kommenden Rinnsales betrug gleichzeitig $7,05-7,1^{0} \mathrm{C}$. - Chemische Zusammensetzungdes Wassersiehe A bsehnitt II (Tabelle).

Die Untersuchung der Proben aus diesem organismenarmen Becken [Pesta 1914] ergab lediglich eine Cladocerenspezies, nämlich:

Chydorus piger G. O. SARS.

\section{Großer Turrachersee.}

(Bisher nicht veröffentlicht.)

Fundortsnum mer: 175. Z 0 n e: Zentralalpen; Norische Alpen. Ge $0 \mathrm{gr}$ a $\mathrm{ph}$ i s e h e L a g e: An der Grenze von. Kärnten und Steiermark auf der Turracherhöhe gelegen. S e e h öh e: $1763 \mathrm{~m}$ ü. d. M. Flu Bge b i et: Predlitzgrabenbach (Turrach)-Mur-Drau. Gr ö ße: ca. $1 \mathrm{~km}$ lang, $500 \mathrm{~m}$ breit. Maximaltiefe: Nicht ermittelt. Geologisehe und hydrographische Daten: Liegt nach den Angaben von Dr. K. Holdhaus in der Kohlenformation zwischen Grauwacke und Tonschiefern. Wass e r a r b e: Dunkel-(schwärzlich-)blau.

In den von Herrn Dr. K. Holdhaus im August des Jahres 1920 aufgesammelten Planktonnetzfängen wurden von uns folgende Formen nachgewiesen:

C o p e p od a: Unbestimmbare Jugendstadien!

Cla d o e e ra: Acroperus harpae BaIRD s. str. Alona affinis (Leydig). Chydorus sphaericus O. F. Müller. Daphnia sp. (iuvenis).

\section{Vassachersee.}

Fundortsnummer: 176. Z o ne: Zentralalpen; Norische Alpen. Ge $0 \mathrm{graph}$ is che Lage: Kärnten, nördlich von Villach. S e ehöh e: ca. $550 \mathrm{~m}$ ü. d. M. Flu Bg ebiet: Drau. Größe: $350 \mathrm{~m}$ lang, $250 \mathrm{~m}$ breit. M a xi malti efe: Nicht genau bekannt, jedoch über $10 \mathrm{~m}$. 
Uther die (rustaceenfauna dieses Sees hat nur Imноғ [1890] Untersuchungen angestellt, deren Ergebnisse Steuer [1897] in sein Verzeichnis aufnahm; es wurden rachgewiesen:

Co pe poda: Cyclops sp.

(Cl a d 0 e e r a: Alona quadrangularis (0. F. Mülder). Bosmina sp. Ceriodaphnia sp. Diaphanosoma brachyurum (Liéviv). Peracantha truncata (O. F. Müller) [Imнof et Steuer sub Pleuroxus tr.]. Sida crystallina (O. F. MÜLLER).

\section{Veldes(er)see.}

Fund ortsnummer: 177. Z o ne: Südliche Kalkalpen; Julische Alpen. Geographische Lage: Krain, bei Veldes am Zusammenfluß der Wurzener Save mit der Wocheiner Save. Se e h öh e: 478 m ü. d. M. F l u Bg e b i e t: Wocheiner Save-Save-Donau. Gr öß e: $1,45 \mathrm{~km}^{2}$ Oberfläche; ca. $2 \mathrm{~km}$ lang und 1,3 km in der größten Breite. M a $x$ i m a l t i e f e: $30,6 \mathrm{~m}$, im Mittel $22 \mathrm{~m}$ tief (nicht $45-46 \mathrm{~m}$ !). Geologische und y drographische Daten: Nach RichTER [1897] eine durch die anstehenden schroffen Kalkberge vor der Zuschüttung bewahrte und ausgesparte Wanne. Langhans [1905] maß am 7. VI. 1904 eine 0 berflä chenwassertemperatur von $18^{\circ} \mathrm{C}$.

Imhof [1890], Langhans [1905], Keissler [1910] und Hartmann [1917], dessen Untersuchungen sich auf die Monate August--September des Jahres 1914 beziehen, fanden hier folgende Formen:

Co p e p o d a: Cyclops sp. [Тмhof, Keissler, Hartmann]. Cyclops serrulatus Fischer [Hartmann 1917]. Cyclops stremuus Fischer [Langinans 1905]. Diaptomus sp. [ImhoF, Langhans]. Diaptomus vulgaris Schmeil [Hartmann 1917].

Cl a d o c e r a: Acroperus angustatus G. O. Sars [Hartmann 1917]. Alona costata G. O. Sars [Hartmann 1917]. Alona rectangula G. O. Sars [Hartmann 1917]. Alona tenuicandis G. O. Sars [Hartmann 1917]. Bosmina sp. [Імhof, Keissler]. Bosmina longirostris (O. F. MüLler) [Langhans 1905; l.-typica Hartmann 1917]. Bosmina longirostriscornuta Jurine [Langhans 1905, Hartmann 1917]. Ceriodaphnia pulchella G. O. Sars [Hartmann 1917]. Daphnia longispina var. cucullata SARS forma apicata (Kurz) [Hartmann 1917]. Daphnia longispina var. cucullata SARS f. kahlbergensis (SchoEdLeR) [IмноF $1890 \mathrm{sub}$ Hyalodaphnia jardinei var. kahlbergensis]. Daphnia longispina var. hyalina forma galeata Sars [Langhans 1905 sub $D$. galeata]. Diaphanosoma 
brachyurum (LiÉvix) [Iyнof. Laxghaxs]. Diaphanosoma brachyurum var. leuchtenbergianum Fischer [HartMaxn 1917]. Leptodora kindtii (Focke) [Imнof 1890]. Peracantha truncata (O. F. Müller) [Hartmann 1917]. Scapholeberis mucronata (O. F. MüLler) fronte laevi et var. cornuta SchoEdLer [HaRTMann 1917].

\section{Walchensee.}

Fu n d o r t s n u m m e r: 178. Z o n e: Nördliche Kalkalpen; Nordtiroler Kalkalpen. Ge ographische Lage: Südbayern (Bezirk Murnau), nördlich von Mittenwald gelegen. S e e h ö h e: $803 \mathrm{~m}$ ï. d. M. Flu Bge bi et: Jacher-Isar-Donau. Gr ö Be: Großes Becken von $8 \mathrm{~km}$ Länge und $6 \mathrm{~km}$ Breite. M a x i malt i e f e: Angeblich $215 \mathrm{~m}$ (196 m nach der Ostalpenkarte des D. und Ö. Alpenvereines). Gre 0 $\mathrm{l} 0 \mathrm{~g}$ i e der Umge bu $\mathrm{ng}$ : Nach Wallmann [1868] zwischen Trias und Lias. Ufer bewaldet. W a s s e $\mathrm{r}$ a $\mathrm{r}$ b e: Dunkelblau. Geistbeck [1885] maß am 1. IX. 1881 eine $\mathrm{Oberflächenwassertem-}$ peratur von $18^{\circ} \mathrm{C}$. - Enthält an $\mathrm{F}$ is ehen: Forelle, Renke, Saibling.

Імноғ [1887], dessen Planktonfänge aus diesem See vom August bis September stammten, und HoFER [1896], welcher hier am 9. und 10. IX. 1895 fischte, melden das Vorkommen folgender Gattungen bezw. Arten:

Copepoda: Cyclops sp. [Імноғ]. Diaptomus sp. [Імноғ]. Diaptomus gracilis G. O. SARS [HoFER].

Cla d o cera: Bosmina sp. [Імноғ]. Daphnia sp. [Імноғ]. Leptodora kindtii (Focke) [Імноғ].

\section{Wallersee.}

Fu n d o r ts n u m mer: 179. Z o n e: Nördliche Kalkalpen; Salzburger Kalkalpen. Ge ogr a ph is e h e La g e: Salzburg, nordöstlich der Stadt Salzburg. S e ehöh e: 504 m ü. d. M. Flu Bge bi e t: Fischbach-Salzach-Inn-Donau. Größ e: 7,52 kn. ${ }^{2}$ Oberfläche; $5,7 \mathrm{~km}$ lang, $1,9 \mathrm{~km}$ breit. M a x i $\mathrm{m}$ a $1 \mathrm{t}$ i e f e: $23,4 \mathrm{~m}$ [FugGer 1890]. Geologisehe und hydrographische Daten: Liegt nach Wallmann [1868] in Molasse (Eocän). Ein Moorsee [Micoletzky 1912]. W a s serf a r be: Lehmgelb (nach Walcmann [1868] bläulich-blafgrün!). Wasserflora reich entwickelt. Zählt zu den wärmsten Seen Salzburgs; als Oberflächenwassertemperaturen wurden im Monate August $19^{\circ}$ C (25. VIII. 1910) bezw. $20^{\circ} \mathrm{C}$ (14. VIII. 1904) 
gemessen [Micoletzky 1912, Brehm 1906]. Fischreich (Barsch, Wels? eingesetzt).

ther die pelagische und litorale Crustaceenfauna des Wallersees gibt die folgende Liste Aufschluß; ihre Zusammenstellung gründet sich auf die Arbeiten von Iмноғ [1885], dessen Planktonfänge vom 2. IX. 1884 stammten, BRenm [1906], welcher am 14. VIII. 1904 in diesem See fischte, und von Micoletzky [1912], dessen Untersuchungen im August 1910 ausgeführt wurden und sich auf die Uferfauna beziehen. Demnach wurden nachgewiesen:

C o p e p o da: Canthocamptus staphylinus (Jurine) [Mrcoletzky]. Cyclops sp. [Імноғ]. Cyclops macrurus G. O. Sars [Micoletzky]. Diaptomus sp. [Імног].

$\mathrm{Cl}$ a d o e e r a: Alona quadrangularis (O. F. Müller) [Micoletzky]. Alona rectangula G. O. SARs [Micoletzky]. Alonella exigua (LilljeborG) [Micoletzky]. Alonella nana (BAird) [Micoletzky]. Alonopsis elongata G. O. Sars [Micoletzky]. Bosmina sp. [Імноf]. Bosmina longirostrisbrevicornis (НеLlich) [ІмноF sub B. brevicornis]. Chydorus sphaericus O. F. Müller [Micoletzky]. Daphnia longispina O. F. Müller [Iмhof]. Diaphanosoma brachyurum (LiÉvin) [Імноғ]. Leptodora kindtii (Focke) [Iмнof]. Peracantha truncata (O. F. Müller) [Brehm, Micoletzky]. Pleuroxus uncinatus BaIRd [Imнof sub $P$. personatus]. Sida crystallina (0. F. Müller) [Imhof, Micoletzky].

\section{Weißsee am Tauernjoch.}

(Bisher nicht veröffentlicht.)

F u n d o r t s n u m mer: 180. Z o n e: Zentralalpen; Hohe Tauern. Geographische Lage: Salzburg, am Kalsertauernjoch (Großglocknergebiet), dem Übergang vom Stubachtal nach Kals in Tirol gelegen. S e e h öh e: $2218 \mathrm{~m}$ ü. d. M. Flu Bg e b i e t: WeißenbachWurfbach-Stub-Ach-Salzach-Inn-Donau. Gr ö B e: $540 \mathrm{~m}$ lang, $420 \mathrm{~m}$ breit. Ma xi malt i e f e: $53 \mathrm{~m}$ [vergl. FugGer 1911]. Ge 0 logische und h ydrographisehe Daten: Liegt im Granitgneis; Böнм [1886] zählt den See zu den echten, durch Erosion entstandenen Felsbecken. Ufer: Flache Moospolsterböden beschränkt (nur im Westen); im übrigen aus Schotter und Felsplatten bestehend, an der Nordseite steile Felswände, zum Teil mit Schutthalden. Der Seegrund selbst steinig (Felsblöcke, Schutt) und sandig. Wa s serfärbung: Milchig-grün, verursacht durch Feinsand. Zahlreiche kleinere Zuflüsse (Schmelzwasser), im Nordwesten ein stärkerer Wasser- 
fall vorhanden. Wasserflora makroskopisch nicht entwickelt, fehlt auch ein Algenbelag an Steinen. Am 24. VIII. 1920 wurde von uns in der Zeit zwischen $9-11^{\mathrm{h}}$ a. m. eine $\mathrm{O}$ ber $\mathrm{fl}$ ä ch e $\mathrm{n}$ w a s ser t e mperatur ron $5-5,1^{\circ} \mathrm{C}$ gemessen; die Temperatur eines der Schmelzwasserrinnsale am Ostufer betrug gleichzeitig $4^{0} \mathrm{C}$. - F is c h e nicht beobachtet. Chemische Beschaffenheit des Seewassers siehe Abschnitt I und II (Tabelle).

Die von uns gesammelten Planktonnetzfänge enthielten keine Cladoceren, sondern ausschließlich C o p e p o d e n, und zwar:

Canthocamptus cuspidatus SchMeIL. Cyclops serrulatus Fischer. Cyclops vernalis Fischer.

\section{Weißensee am Fernpaß.}

F u n d ort s n u m mer: 181. Z o n e: Nördliche Kalkalpen; Nordtiroler Kalkalpen. Ge ograph is che Lage: Nordtirol, nahe der Fernpaßhöhe nördlich von Imst. S e e h öh e: 1085 m ü. d. M. Flu B g e b i e t: Loisach-Isar-Donau. Gr ö B e: ca. $450 \mathrm{~m}$ lang und etwa $200 \mathrm{~m}$ breit. Maximaltiefe: Unbekannt. Ge ologische und h y drogra ph is che Dat e n: Der See liegt in Moränenschutt und gehört nach Böнм [1886] gleich den übrigen Seen am Fernpaß zu den durch Einbruch oder Auflösung des Kalkes entstandenen Einsturzbecken. Geist beck [188ว̃] maß am 13. VIII. 1881 eine 0 b e r f l ä c h e nwas sertemperatur von $15,3^{\circ} \mathrm{C}$.

Dieses Wasserbecken untersuchte BreHм [1907] auf Plankton; er meldet jedoch das Fehlen von Planktonorganismen im See, welches Ergebnis als sehr unwahrscheinlich bezeichnet werden muß, zudem hier nach Dalla Torre [1913] mehrere Fische (Brachse, Forelle, Pfrille, Saibling) vorkommen. Vergl. dazu die Fundorte Nr. 13, (26), 27, 32, $33,51,122,123,151$ und 167 , welche ebenfalls von BREHM untersucht worden sind!

\section{Weißensee bei Füssen.}

F u n d o r t s n u m mer: 182. Z o n e: Nördliche Kalkalpen; Vilsergruppe der Nordtiroler Kalkalpenkette. Ge o graphis che Lage: Südbayern, westlich von Füssen. S e e h ö h e: 803 m ü. d. M. Flu B g e bi e t: Lech-Donau. Größe: $2,4 \mathrm{~km}$ lang, $700 \mathrm{~m}$ breit. M a ximaltiefe: Unbekannt. Geologische und hydrographische Daten: Unbekannt. - Enthält Forellen.

Імноғ [1887] stellte in seinen Planktonfängen vom August bis September folgende $\mathrm{Cl}$ a d o c er en fest: 
Bosmina sp. Daphnia sp. Diaphanosoma brachyurum (LiÉviN). Leptodora kindtii (FockE).

\section{Weißensee bei Greifenburg.}

F u n d o r ts n u m mer: 183. Z o n e: Südliche Kalkalpen; Gailtalergruppe der Karnischen Alpen. Geographisehe Lage: Kärnten, südlich des Drautales bei Sachsenburg, östlich von Greifenburg gelegen. S e eh öh e: 926 m ü. d. M. Flu Bg e b i e t: WeißenbachDrau. Größe: Gestrecktes Wasserbecken von etwa $12,5 \mathrm{~km}$ Länge und nur $1 \mathrm{~km}$ größter Breite. M a x i m a l t i e f e: $97 \mathrm{~m}$. G e $0 \mathrm{l} 0 \mathrm{~g}$ is ch e u nd h y drographische Date n: Uns nicht bekannt; er erfüllt nach Wallmann [1868] eine diluviale oder tertiäre Talspalte; HalbFass [1913] vergleicht den See infolge seines nicht zum nächstgelegenen Punkt der Drau entströmenden Abflusses mit dem Achensee in Tirol (Nr. 1). Nach Brehm und Zederbauer [1905] charakterisiert sich der Weißensee durch den Mangel an Phytoplankton und Rotatorien!

Steuer [1897] und Brehm und Zederbauer [1905] zählen folgende Spezies für diesen Fundort auf:

Co p e poda: Cyclops leuckarti Claus [Steuer, Brehm und Zederbauer]. Cyclops strenuus Fischer [Steuer].

$\mathrm{Cl}$ a d o e e r a: Bosmina coregoni-longispina LEyDig forma bohemica Sting. [Steuer sub B. bohemica]. Bosmina longirostris (O. F. Müller) [Brehy und Zederbauer]. Ceriodaphnia pulchella G. O. Sars [Steuer]. Ceriodaphnia quadrangula (O. F. Müller) [Brehm und Zederbauer]. Diaphanosoma brachyurum (LiÉvin) [BREHM und ZEDERBAUER].

\section{Oberer Weißenfelsersee.}

Fundortsnummer: 184. Z o n e: Südliche Kalkalpen; Jrlische Alpen. Ge o graph is ch e Lage: Krain, östlich von Tarvis am Nordhang des Mangart (Manhart), südlich vom Orte Weißenfels. S e eh öh e: $940 \mathrm{~m}$ ü. d. M. Flu Bg e bi e t: Ursprungsgebiet der Gailitz-Gail-Drau. Größ e: $550 \mathrm{~m}$ lang, $375 \mathrm{~m}$ breit. Ge 0 l $0 \mathrm{gische} u n d \mathrm{~h}$ y drographische Daten: Nicht bekannt.

KEISSLER [1910] erwähnt keine Zooplanktonvertreter; hingegen ergaben die während der Monate August-September des Jahres 1914 ausgeführten Untersuchungen von HARTMANN [1917] das Vorkommen folgender Arten:

Co p e po d a: Cyclops serrulatus Fischer. Cyclops viridis Jurine. 
Cl a d o cera: Alona quadrangularis (O. F. MïLler). Chydorus sphaericus (O. F. MüLleR). Eurycercus lamellatus (O. F. MÏLler). Simocephalus vetulus (O. F. MëLLER).

\section{Unterer Weißenfelsersee (= Unterer Mangartsee).}

Fundortsnum mer: 185. Z o n e: Südliche Kalkalpen; Julische Alpen. Ge ographische Lage und Flubgebiet wi c bei Fundortsnummer 184. Seehöhe: $930 \mathrm{~m}$ ü. d. M. Größ e: $500 \mathrm{~m}$ lang, $225 \mathrm{~m}$ breit. Ge $0 \mathrm{log}$ is che und h ydrogr a phis che Date $n$ : Uns nicht bekannt.

Diesen See untersuchte zuerst Breнм [1906], der unter dem Namen „Unterer Mangartsee" zweifellos den Unteren Weißenfelsersee meint; er fand hier nur „Fragmente“. Auch Keissler [1910] macht keine Angaben über das Zooplanktor. Zuletzt urtersuchte den See im August bis September 1914 Hartmann [1917], dessen Planktonfänge ebenfalls. weder Diaptomiden roch Cladoceren enthielten, sondern lediglich die Gattung Cyclops sp. (Die Spezies konnte nicht bestimmt werden.)

\section{Wildsee bei Seefeld.}

F u n d o r t s n u m m e r: 186. Z o n e: Nördliche Kalkalpen; Nordtiroler Kalkalpen. G e $0 \mathrm{~g} r$ a $\mathrm{ph}$ is c h e L a g e: Nordtirol, nordwestlich ron Innsbruck bei Seefeld an der Karwendelbahn. S e e h öh €: $1180 \mathrm{~m}$ ï. d. M. Fl u ß g e b i e t: Drahnbach-Gießer-Isar-Donau. G r ö B e: ca. $660 \mathrm{~m}$ lang und $150 \mathrm{~m}$ breit. M a x i ma $\mathrm{lt}$ i e $\mathrm{fe}: 16 \mathrm{~m}$ [GEISTBECK 1885]. Geologische und hydrographische Daten: Böнм [1886] zählt den See zu den durch Schuttkegel und Muhrbrüchen entstandenen Staubecken. Ufer sumpfig. Enthält nach Dalla Torre [1913]: Hecht, Karpfen, Schleie.

Die Angaben über die Crustaceenfauna dieses Sees sind sehr spärlich und zerstreut; Steuer [1901], Brehm und Zederbauer [1902 und 1906] erwähnen je eine Spezies, nämlich:

Co p e poda: Cyclops fuscus Jurine [Brehm und Zederbaler 1906]. Heterocope saliens LILLJEBorg [STEuer].

Cla d o c e r a: Scapholeberis mucronata var. cormuta (SCHOEDLER) [BREHM und ZeDERBaUER 1902].

Wildsee am Wildseeloder (= Wildalpsec).

Fundortsnum mer: 187. Z on e: Zentralalpen; Kitzbühlergruppe der Salzburger Schieferalpen. Ge ographisch e Lage: 
Tirol, östlich von Kitzbühel am Fuße des Wildseeloder (2119 m) gelegen. S e e h ö h e: Nach Dalla Torre [1913] 2029 m ü. d. M.; seine Angabe "gilt als der höchstgelegene See Österreichs" kann sich wohl nur auf cine Volksmeinung der nächsten Umgebung beziehen! F l u Bg e b i e t: Pillerseeache-Große Ache-Alz-Inn-Donau. G r ö B e: $400 \mathrm{~m}$ lang, $300 \mathrm{~m}$ breit. Tief e: Unbekannt. Ge olog is che und hydrographische Daten: Fehlen. Zweifellos ein echtes Felsbecken. Enthält Fis che (Forelle, Saibling).

Brehm [1907] fand außer Rotatorien eine C y c lo ps-Spezies, die er als „vermutlich"strenuus Fischer ( $i$ uvenis) bezeichnet.

\section{Wildensee ( $=$ Wildsee).}

Fu n d o r t s n u m mer: 188. Z o n e: Nördliche Kalkalpen; Salzkammergutgebiet. Ge o gra ph is ch e L a g e: Nahe der Grenze von Steiermark und Oberösterreich, im Toten Gebirge südlich vom Offensee (Nr. 114) gelegen. S e e h öh e: $1554 \mathrm{~m}$ ü. d. M. Flu Bg e biet: Offenseebach-Traun-Donau. Grö B e: $0,07 \mathrm{~km}^{2}$ Oberfläche; $420 \mathrm{~m}$ lang, $250 \mathrm{~m}$ breit. Maxi malt i e fe: $40 \mathrm{~m}$.

Langhans [1911] gibt für diese Fundstelle nur das Vorkommen einer Cladocere an, nämlich: A lon a gut tata G. O. SARS.

\section{Wocheinersee.}

Fund ortsnummer: 189. Z.o ne: Südliche Kalkalpen; Julische : Alpen. G e o g r a ph is ch e Lag e: Krain, südlich vom Gebirgsstocke des Triglav. S e e h öh e: $526 \mathrm{~m}$ ü. d. M. Flu B g e bi et: Wocheinersave-Save. G r ö B e: $3,28 \mathrm{~km}^{2}$ Oberfläche; über $4 \mathrm{~km}$ lang, größte Breite ungefähr $1,1 \mathrm{~km}$. M a ximalti efe: $44,5 \mathrm{~m}$ (nicht $69 \mathrm{~m}$ !), im Mittel 29,7 m tief. Ge ologis ehe und hydrogra ph is ch e Dat e n: Richter [1897] stellt den Wocheinersee zu den Kalkalpenseen vom Typus des Königs-, Hallstätter- oder Gosausees, denen er auch durch die Umrahmung von hohen Felswänden gleicht; er gehört zu den durch Schuttkegel und Moränenbildung abgedämmten Stauseen. W a s s e r fär bu ng: Tiefblau. Übrige Daten unbekannt.

Nach Iмноғ [1890], Langhans [1905], dessen Fänge vom 27. VIII. 1903 und vom Mai 1904 stammten, und KeIssler [1910] wurden im Wocheinersee folgende Formen nachgewiesen:

Copepoda: Cyclops sp. [Imнof, Keissler]. Cyclops strenuus Fischer [Langhans]. Diaptomus laticeps G. O. Sars. 
$\mathrm{Cl}$ a d o c e r a: Acroperus harpae Baird [Iмноғ sub A. leucocephalus]. Bosmina sp. [Imhof, Keissler]. Bosmina coregoni-longicornis Schoedler [Langhans sub B. longicornis]. Chydorus sphaericus O. F. Müller [Langhans]. Daphnia sp. [Langhans]. Diaphanosoma brachyurum (Liévin) [Imhof, Langhans]. Monospilus dispar G. O. Sars [Imhof sub M. tenuirostris]. Scapholeberis mucronata (O. F. MÜLler) [Langhans].

\section{Wolfgangsee (= Abersee).}

Fu nd ortsn u m mer: 190. Z o n e: Nördliche Kalkalpen; Salzkammergutgebiet. Ge o g r a ph i s ch e L a ge: Salzburg, westlich von Ischl. S e eh öh e: $539 \mathrm{~m}$ ü. d. M. Flu Bge b i e t: Isehl-TraunDonau. Gr ö ße: 10,5 km lang, $2 \mathrm{~km}$ breit; $13,15 \mathrm{~km}^{2}$ Oberfläche. M a ximalti efe: $114 \mathrm{~m}$, im Mittel 47,1 m tief. Ge ologis che und h ydrographisehe Daten: Liegt in Lias- und Gosauschichten; nach Halbfass [1913] eine in festes Gestein eingesenkte Felswanne (Zungenbecken). W a s s e rf a r b e: Grün-blau. Brenm und ZederBAUER [1906] geben für den 30. XII. 1901 eine Wassertemperatur von $4^{0} \mathrm{C}$ (Oberfläche $-10 \mathrm{~m}$ Tiefe) an; am 24. III. 1902 wurde von ihnen eine Oberflächenwassertemperatur von $3^{0} \mathrm{C}$ (in $5 \mathrm{~m}$ Tiefe $4^{0} \mathrm{C}$ ) gemessen. - Enthält folgende F is c h e: Abramis brama, Alburnus lucidus, Barbus fluviatilis, Coregonus wartmanni, Cottus gobio, Esox lucius, Gobio fluviatilis, Lota vulgaris, Perca fluviatilis, Salmo salvelinus, Scardinius erythrophthalmus, Squalius cephalus, Tinca vulgaris und Trutta lacustris.

Die folgende Liste der Gattungen und Arten gründet sich auf die Untersuchungen von Iмноғ [1885] (Fänge vom 26. VIII. 1884), KeIssLeR [1901] (Fänge vom 2. IV. 1901), Keissler [1902] (Fänge vom Juni bis Juli und August bis September 1901), Brehm und Zederbauer [1906] (Fänge vom 30. XII. 1901 und 24. III. 1902) und Langhans [1911]. Es wurden insgesamt nachgewiesen:

C o p e p o d a: Cyclops sp. [Imнof, Keissuer 1902]. Cyclops strenuus Fischer [Keissler 1901, Brehm und Zederbauer]. Diaptomus sp. [ImhoF, Keissler 1902]. Diaptomus gracilis G. O. Sars [KeissLer 1901 sub D. graciloides; Brehm und Zederbauer].

Cl a d o c e r a: Bosmina sp. [Inнof, Keissler 1902]. Bosmina coregoni Baird [Brehm und Zederbauer]. Bosmina coregoni-longispina LEYdig forma bohemica Sting. [KeIssler 1901]. Bythotrephes longimanus Leydig [Imhof, Keissler 1902, Langhans]. Daphnia sp. [Imhof, KeIssler 1902]. Daphnia longispina var. cucullata Sars forma kahlbergensis Schoedler [ImнoF sub D. kahlbergensis]. Daphnia longispina 
var. cucullata forma sarsi Langhans [Langhans]. Daphnia longispina var. hyalina Leydig [Brehm und Zederbauer, Langhans]. Diaphanosoma brachyurum var. leuchtenbergianum Fischer [Langhass]. Leptodora Kindtii (Focke) [Imhof, Keissler 1902, Langhans]. Sida crystallina (O. F. Müller) [Langhans].

\section{Worstnigsee ( $=$ Worstsee $)$.}

Fu n d o r t s n u m mer: 191. Z o n e: Südliche Kalkalpen; Klagerfurter Becken. Gre o graphis che Lage: Kärnten, nördlich des Wörthersees (zwischen Pörtschach und Velden) gelegen. S e ehöh e: ca. $630 \mathrm{~m}$ ü. d. M. Flu Bg e b i e t: Fließt in den Wörthersee (Gebiet der Drau) ab. Gr ö ß e: Größter Längendurchmesser ca. $850 \mathrm{~m}$; ca. $480 \mathrm{~m}$ breit. M a ximalti efe: $30 \mathrm{~m}$. Übrige Daten nicht bekannt.

Iмноғ [1890], Steuer [1897], welcher lediglich IмноF's Angaben zitiert, und Keissler [1906], dessen Angaben sich nur auf Genera bcziehen, stellten für diesen See folgende Formen fest:

Co p e po da: Cyclops sp. [Imhof, Steuer, Keissler]. Diaptomus sp. [Iмhof, Steuer, Keissler].

Cla d o e era: Bosmina sp. [Imhof, Steuer]. Ceriodaphnia sp. [Імнof, Steuer]. Daphnia sp. [Імноғ, Steuer, Keissler]. Diaphanosoma brachyurum (LiÉvin) [IмноF, Steuer]. Leptodora kindtii (Fоске) [Imhof, Steuer]. Sida crystallina (O. F. Müller) [Imhof, Steuer].

\section{Wörthersee.}

F u n d o r t s n u m m e r: 192. Z o n e: Südliche Kalkalpen; Klagerfurter Becken. Ge $0 \mathrm{graph}$ is che Lage: Kärnten, westlich von Klagenfurt. S e e h ö h e: 439 m ü. d. M. F l u B g e b i e t: Glan-GurkDrau. Gr ö ß e: $17 \mathrm{~km}$ lang, durchschnittlich über $1 \mathrm{~km}$ breit; $19,437 \mathrm{~km}^{2}$ Oberfläche. Maximalti efe: In der westlichen Hälfte des Beckens $84,6 \mathrm{~m}$, in der östlichen Hälfte 73,3 m; mittlere Tiefe des ganzen Wörthersees $43,2 \mathrm{~m}$. Ge $0 \mathrm{log}$ is che und hydrographis che Daten: Richter [1897] sagt: „Die Umgebung des Sees ist durchwegs eine echte Glaziallandschaft. Der See selbst ist zwischen Schieferberge und Hügel eingelagert, die zum Teil direkt seine Ufer bilden, zum Teil noch als Klippen aus den Alluvionen hervorragen, die sich neben dem See hirziehen. Diese kleinen Hügel und Rücken aus festem Schiefer, die man auf der Karte für Moränen halten könnte, sind geradezu charakteristisch für die östliche Seehälfte . . B Breite Entwicklung der Uferbank mit Schilfwuchs tritt an zahlreichen Orten auf." Ostufer stark verflacht 
und auf $100-200 \mathrm{~m}$ breitem Streifen verschilft. B o d e $\mathrm{n} b \mathrm{es}$ e h a f f e nh e it: Seegrund schlammig. Wa s s e r t e mp e r a t u r messung e n vergl. Richter [1897]; in den Sommermonaten Juli und August wurden Oberflächenwassertemperaturen bis zu $27^{\circ}$ C beobachtet. -. Entlält folgende F is e h e: Alburnus lucidus, Coregonus wartmanni, Esox lucius, Lota vulgaris, Perca fluviatilis, Rhodeus amarus, Scardinius erythrophthalmus, Silurus glanis, Tinca vulgaris und Trutta lacustris.

Nach den Untersuchungen von Imhof [1890], Steuer [1897], Brehm und Zederbauer [1905] und Keissler [1906] setzt sich die Copepodenund Cladocerenfaura des Wörthersees aus folgenden Formen zusammen:

Copepoda: Canthocamptus staphylinus Jurine [Steuer]. Cyclops sp. [Imнof, Keissler]. Cyclops leuckarti Clads [Steuer]. Cyclops macrurus G. O. Sars [Steuer]. Cyclops serrulatus Fischer [Steuer]. Cyclops strenuus Fischer [Brehm und Zederbauer]. Diaptomus sp. [Imhof, Keissler]. Diaptomus gracilis G. O. Sars [Steder. Brehy und Zederbauer].

Cla d o e e ra: Acroperus harpae Batrd [Steuer sub A. leucocephalus]. Alona affinis (Leydig) [Steuer]. Bosmina sp. [Imнof. Keissler]. Bosmina coregoni Baird [Brehy und Zederbauer]. Bosmina coregoni-longicornis SCHOEDLER [STEUER sub B. longicornis]. Ceriodaphnia pulchella SaRs [Steuer]. Ceriodaphnia reticulata (JuRINe) [Steder]. Daphnia sp. [Imhof, Keissler]. Daphnia longispina var. cucullata Sars [Brehy und Zederbauer sub Hyalodaphnia c.]. Daphnia longispina rar. cucullata forma incerta (RICHARD) [STEUer sub Hyalodaphnia jardinei rar. incerta]. Daphnia longispina rar. cucullata forma kahlbergensis Schoedler [Iyнof, Steuer sl b D. kahlbergensis]. Daphnia longispina var. hyalina forma galeata Sars [Iмноғ sub D. galeata]. Daphnia longispina rar. longispina forma caudata Sars [SteUer sub D. $l$. var. caudata]. Diaphanosoma brachyurum (Lrévin) [Iмhof, Steuer. Brehy und Zederbader]. Eurycercus lamellatus (O. F. Müller) [Steuer]. Leptodora kindtii (Focke) [Imhof. Brehis und Zederbacer, Keissler]. Simocephalus vetulus (O. F. Müller) [Steuer].

Würmsee siehe Starnbergersee (Nr. 159).

\section{Zellersee $\left.{ }^{1}\right)$.}

Fu n d o r t snu mmer: 193. Z o ne: Zentralalpen; Salzburger Schifferalpen. Ge ogr a ph is ch e Lag e: Salzburg, bei Zell am See.

1) Der Fundort liegt im Pinzgau (Salzburg) und ist nicht zu verwechseln mit dem Zellersee im Salzkammergut (Oberösterreich), der allerdings meist Irrsee genannt wird. 
S e e h öh e: 749,6 m ü. d. M. F l u ßg e bi et: Salzach-Inn-Donau. Gr ö B e: 4,7 kn ${ }^{2}$ Oberfläche; $3,8 \mathrm{~km}$ lang, größte Breite $1,5 \mathrm{~km}$. M a $\mathrm{xim}$ a l t i efe: $69,5 \mathrm{~m}$, mittlere Tiefe $37 \mathrm{~m}$. Ge ol o g i s ch e u nd h y drograph is ch e Dat e $n$ : Liegt zwischen kristallinen Schiefern. Nach Böнм [1886] ein durch Terrassenschotter gestauter See. HalBFASS [1913] hält ihn für ein Stück übertieften Tales, welches zwischen zwei größeren Flüssen erhalten geblieben war und durch Bifurkation des Gletschers bedingt ist. Längsufer auf der Ost- und Westseite steil, der Nord- und Südrand des Sees ganz verflacht, sumpfig und moorig; hier ist auch die Ufervegetation reicher entwickelt als an den übrigen Stellen. Von Mikrophyten hat im Zellersee das Auftreten der sogenannten „Seeknödel“ (Aegagropila) besondere Beachtung gefunden. Phytoplanktonformen vergl. Keissler 1910 [in: Archiv f. Hydrobiol., vol. 5, p. 339]. W a s s e r t e m p e r a t u r e n vom 21. XII. 1901 nach BREHM und Zederbauer [1906]: $1^{0} \mathrm{C}$ an der Oberfläche, in 5-15 m Tiefe $4^{0}$ C. Micoletzky [1912] stellte am 7. VIII. 1910 folgende Wassertemperaturen fest: Oberfläche $17,2^{\circ} \mathrm{C}$, in $10 \mathrm{~m}$ Tiefe $12,4^{\circ} \mathrm{C}$, in $20 \mathrm{~m}$ Tiefe $6,8^{\circ} \mathrm{C}$ und in $63 \mathrm{~m}$ Tiefe $6^{\circ} \mathrm{C}$. - W a s s e $\mathrm{r}$ f a r be: Grün.

Die Crustaceenfauna des Zellersees haben Iмноғ [1885], Вrenм und Zederbauer [1906], Micoletzky [1912] und zuletzt Hartmann [1917] untersucht; die beiden letzten Autoren haben eingehend über die Litoralfauna berichtet, so daß das Bild über die gesamte Copepodenund Cladocerenfauna ziemlich vollständig sein dürfte. Es wurden im ganzen folgende Formen nachgewiesen:

C o p e p o d a: Canthocamptus schmeili var. biserialis [MICOLETzKy]. Cyclops sp. [Imhof]. Cyclops albidus Jurine [Micoletzky, Hartmann]. Cyclops leuckarti Claus [Brehm und Zederbauer, Micoletzky]. Cyclops macrurus SARs [Micoletzky]. Cyclops serrulatus Fischer [Micoletzky]. Cyclops strenuus Fischer [BRehm und Zederbauer]. Cyclops viridis Jurine [Micoletzky]. Diaptomus sp. [Iмнof]. Diaptomus gracilis SARS [BREhM und Zederbauer].

Cla do cera: Acroperus angustatus G. O. Sars [Hartmann]. Acroperus harpae BAIRD [Micoletzky, Hartmann]. Alona costata G. O. Sars [Micoletzky, Hartmann]. Alona rectangula G. O. Sars [Hartmann]. Alonella exigua (Lillueborg) [Hartmann]. Bosmina sp. [IMHOF]. Bosmina longirostris (0. F. MülLer) [Brehm und Zederbauer]. Bythotrephes longimanus Leydig [Brehm und Zederbauer]. Ceriodaphnia sp. [BRehm und Zederbauer]. Ceriodaphnia pulchella G. O. SarS [Micoletzky]. Chydorus gibbus Lilljeborg [Micoletzky]. Chydorus 
sphaericus O. F. Müller [Mrcoletzky, Hartmani]. Chydorus sphaericus var. coelatus Sting. [Hartmanv]. Daphnia sp. [Iмнof]. Daphnia longispina var. hyalina Leydig [Brehy und Zederbauer sub D. hyalina]. Diaphanosoma brachyurum (LiÉviv) [HaRTMANv]. Eurycercus lamellatus (O. F. MÜller) [Micoletzky]. Monospilus dispar G. O. Sars [MicoLetzky]. Peracantha truncata (O. F. Müller) [Micoletzky, Hartmany]. Pleuroxus uncinatus BAIRD [Micoletzky]. Scapholeberis mucronata (0. F. Müller) [Micoletzky, Hartmany]. Sida crystallina (0. F. MülleR) [Micoletzky, Hartmann].

\section{Zireinersee.}

(Bisher nicht veröffentlicht.)

F u n d o r t s n u m mer: 194. Z o n e: Nördliche Kalkalpen; Nordtiroler Kalkalpen. Ge o gra ph is e h e L a ge: Nordtirol, nördlich von Brixlegg im Unterinntale im Gebiete der Rofanspitze gelegen. S e ehöh e: $1793 \mathrm{~m}$ ü. d. M. Flu Bg e bi et: BrandenbergeracheInn-Donau. Gr ö ße: ca. $\left.800^{1}\right) \mathrm{m}$ lang, $\left.400^{1}\right) \mathrm{m}$ breit. M a x i ma l t i e f e: $200 \mathrm{~m}$ [Angabe nach Dalla Tonre 1913]. G e o l o g i s ch e u n d h ydrographische Daten: Liegt in Kössener Schichten. Ufer meist steilabfallende Hänge und Schuttkare, an einigen Stellen flacher und schwach versumpit. Enthält: For ellen. Von uns wurden zahlreiche Fische beobachtet.

Die am 31. VII. 1921 gesammelten Planktonnetzfänge enthielten folgende Formen:

Co p e poda: Cyclops vernalis Fischer. Diaptomus sp. (iuvenis).

Cl a d o c e ra: Acroperus harpae BaIRd. Alona affinis (Leydig). Chydorus sphaericus (O. F. MüLlER).

\section{Zirmsee.}

F u n d o r t s n u m mer: 195. Z o n e: Zentralalpen; Hohe Tauern. Ge $0 \mathrm{gr}$ a ph i s c h e L a ge: Nordkärnten, östlich von Heiligenblut im Gebiete der Hochnarrspitze gelegen. Se ehöh e: $2500 \mathrm{~m}$ ü. d. M. Fl u ßg e bi e t: Möll-Drau. Größe: Hochgebirgsbecken von geringem Ausmaße, genaue Angaben nicht festzustellen, ebenso die übrigen Daten nicht bekannt.

1) Die bei Dalla Torre [1913] angeführten Ausmaße "80 m lang, $40 \mathrm{~m}$ breit" beruhen offenbar auf einem Druckfehler; uns scheint auch seine Tiefenangabe (200 m) höchst unwahrscheinlich und soll hier vielleicht bloß $20 \mathrm{~m}$ stehen! 
Brenm [1907] fand in seinen vom 18. August stammenden Planktornetzfängen folgende zwei Spezies:

Cyclops strenuus Fischer und Chydorus sphaericus (O. F. MüLler).

\section{Nachtrag.}

Nach vollständigem AbschluP des Manuskriptes dieses Abschnittes rrsehien eine Bearbeitung des Planktons des Ammersees von Georg Fritz [Zoolog. Jahrb. f. System., vol. 44, Heft 6, p. 487; 1922], deren Ergebnisse hier zur Ergänzung angefügt werden müssen.

\section{Ammersee.}

Fu n d ortsnummer: 196. Z o ne: Voralpenbereich der Nördlichen Kalkalpen. Ge ograph is ehe Lage: Südbayern, westlich ron München. S e e h öh e: $533 \mathrm{~m}$ ü. d. M. Fl u B g e b i e t: AmmerAmper-Isar-Donau. Gr ö ße: $16 \mathrm{~km}$ lang, $6 \mathrm{~km}$ breit. M a x i m a lt i e f e: $79 \mathrm{~m}$.

Georg Fritz [1922] führt folgende Formen an:

C o p e p o d a: Cyclops sp. Diaptomus sp.

Cl a d o c e ra: Alonella nana (BAIRD). Bosmina coregoni (kessteri UlJanin). Bosmina longirostris (brevicornis Hellich). Bythotrephes longimanus LEYdig. Daphnia longispina var. hyalina f. galeata SARS [Fritz sub D. l. var. galeata]. Daphnia longispina var. hyalina f. pellucida P. E. MüLler. Diaphanosoma brachyurum LiÉvin. Leptodora kindti (Focke). Pleuroxus uncinatus BAIRD.

Ferner wurde uns nach Einsicht in die Abhandlung von Langhans [1908, Naturw. Zeitschrift „Lotos“ (Prag), vol. 56, p. 209 und 255] ein weiterer Fundort im Bereiche der Ostalpen bekannt, nämlich:

\section{Mittersee am Schafberg.}

Fu n d ortsnu m mer: 197. Z o n e: Nördliche Kalkalpen; Salzkammergutgebiet. Ge $0 \mathrm{gr}$ a $\mathrm{ph}$ is $\mathrm{ch}$ e L a g e: An der Grenze zwischen Oberösterreich und Salzburg; am Schafberg gelegen. S e ehöh e: $1334 \mathrm{~m}$ ü. d. M. Flu Bge bi et: Ischl-Traun-Donau. Gr öße: $100 \mathrm{~m}$ im Durchmesser. Ma $\mathrm{ximalt}$ i e $\mathrm{fe:} 7,6 \mathrm{~m}$. - Langhans [1908] fand hier Heterocope saliens LiLlJeborg. 


\section{Abschnitt.}

\section{Über die Verbreitung und das Auftreten der Copepoden und Cladoceren in den Seen der Ostalpen.}

Die im folgenden eingehaltene Anordnung der Genera, Spezies und Varietäten in der Reihenfolge des Alphabetes soll lediglich der Forderung nach einer größeren Übersichtlichkeit und Bequemlichkeit beim Nachschlagen gerecht werden.

\section{Copepoda.}

\section{Canthocamptus.}

Von diesem Genus sind bisher 12 Spezies und 3 Varietäten aus den untersuchten Ostalpenseen bekannt geworden. Sie gehören durchwegs zur Litoralfauna oder leben in größeren Tiefen der Wasserbecken am Grunde und im Schlamm. Die Nachweise von ihrem Vorkommen müssen noch immer als sehr sporadisch bezeichnet und dementsprechend auch bewertet werden; für die meisten Spezies wird im Laufe der Zeit eine bedeutend vermehrte Zahl von Fundorten zu nennen sein. Die Vertreter dieser Gattung derzeit von allen tiergeographischen Betrachtungen gänzlich auszuschließen, scheint uns deshalb dringend geboten. Die aus diesen "Studien“ resultierenden Verbreitungsdaten können nur als eine erste, bescheidene Grundlage für später dienen. Über die Systematik der Formen orientiert am besten Haberbosch [1917].

Ohne Nennung der Spezies wird das Vorkommen von Canthocamptus aus 3 Seen der Zentralalpenzone gemeldet, nämlich aus dem Königsangersee (2173 m ü. d. M.), dem Millstättersee (580 m ü. d. M.) und dem Unteren Mühldorfersee (2281 m ü. d. M.).

1. Canthocamptus crassus G. O. SARS.

Bisher nur aus dem Litorale des Faistenauer Hintersees (Nördliche Kalkalpenzone) bekannt.

2. Canthocamptus cuspidatus SchmeiL.

Fundorte: Achensee (Nördliche Kalkalpen); Oberer und Unterer Sonntagskarsee, Weißsee am Tauernjoch (Zentralalpen). Sie gehören sämtlich zu den kalttemperierten Seen und liegen in Höhen von 930-2218 m ü. d. M. Dieses Vorkommen im Ostalpenbereich stimmt mit den Angaben aus den Schweizer Alpen gut überein, da die von ThuÉBAUD [1915] für C. cuspidatus genannten Fundorte (5) alle in Höhen von $1810-2456 \mathrm{~m}$ liegen. 
3. Canthocamptus echinatus var. luenensis SchmeIL.

Bisher nur aus dem Lunzer Untersee, Lünersee (Nördl. Kalkalp.), aus Tiefen von $20-34 \mathrm{~m}$ gemeldet.

4. Cantho camptus hofer $i$ van Douwe.

Bisher nur für den Attersee und Bodensee (Nördl. Kalkalp.) nachgewiesen; im ersteren bodenbewohnend, in Tiefen von 27-107 m.

5. Canthocamptus microstaphylinus Wolf.

Aus dem Litorale des Faistenauer Hintersees (Nördl. Kalkalp.) gemeldet.

6. Canthocamptus minutus Claus.

Fundorte: Bodensee, Eibsee, Lünersee (Nördl. Kalkalp.); Gardasee, Lasessee, Piazzesee, Stellunesee (Südl. Kalkalp.). In den Seen der Zentralalpenzone bisher nicht nachgewiesen. Im Gardasee in 40-70 $\mathrm{m}$ Tiefe beobachtet. Von den genannten 6 Wasserbecken stellt der Piazzesee mit 19 m Tiefe den seichtesten dar. Höchstgelegener Fundort: Stellunesee $(2140 \mathrm{~m})$.

7. Canthocamptus rha eticus SCHMEIL.

Bisher in folgenden Seen der Nördlichen Kalkalpenzone nachgewiesen: Gafiensee, Lunzer Obersee, Lunzer Untersee, Lünersee, Partnunersee, Tilisunasee. - So zeigt das einstweilen gewonnene Verbreitungsbild die Eigentümlichkeit, daß je zwei Fundstellen nahe beisammen liegen (Gafien-, Lüner- und Partnunersee-Lunzer Ober- und Untersee); diese zwei Verbreitungsfelder jedoch wie zwei Inseln durch die ganze Länge der Ostalpen weit voneinander getrennt sind. Mit Ausnahme des Lunzer Untersees liegen die Seen in Höhen von 1113-2312 m ü. d. M.

8. Canth o camptus s chmeili MrazeK.

Bisher nur im Mittleren Klippensee (Zentralalp.), Bodensee und im. Lunzer Untersee (Nördl. Kalkalp.) gefunden.

9. Canth o c a mptus schme $i l i$ var. $b i s$ er $i$ a $l i s$ Micoletzky.

Nur zwei Fundorte, Attersee (Nördl. Kalkalp.) und Kampspitzsee (Zentralalp.) bekannt; aus dem ersteren auch aus Tiefen von 13-107 m nachgewiesen. Nach ThiÉBaud [1915] in der Schweiz nicht gefunden.

10. Canth o camptus schmeili var. ha mata SchmeIL.

Bisher nur aus dem Lünersee (Nördl. Kalkalp.; 1943 m ü. d. M.) gemeldet.

11. Canthocamptus staphylinus (JURINE).

Fundorte: Faistenauer Hintersee, Hallstättersee, Lunzer Mittersee, Lunzer Obersee, Lunzer Untersee, Wallersee (Nördl. Kalkalp.); Großer Magdalenensee, Wörthersee (Klagenfurter Becken der Südl. Kalkalp.); 
Angaben über das Vorkommen in den Zentralalpen fehlen bisher. Höchstgelegener Fundort: Lunzer Obersee (1113 m ü. d. M.). Die Form aus dem Lunzer Untersee gehört vielleicht einer nov. var. an.

12. Canthocamptus trispinosus BRADY.

Aus dem Litorale des Faistenauer Hintersees (Nördl. Kalkalp.) bekannt. Fehlt nach Thí́Baud [1915] in den Schweizer Alpen.

13. C a n tho camptus vejdovskyi MrazeK.

Nur aus dem Lunzer Untersee (Nördl. Kalkalp.) gemeldet.

14. Canthocamptus wierzejskii Mrazek.

In folgenden Seen der Nördlichen Kalkalpenzone nachgewiesen: Attersee, Erlaufsee, Grundlsee, Hallstättersee, Lunzer Untersee. Alle Fundorte gehören zu den Alpenseen mit größeren Tiefen (170,6 m, 44 m, $63,8 \mathrm{~m}, 125,2 \mathrm{~m}, 35 \mathrm{~m})$; im Attersee wurde die Spezies in $53-107 \mathrm{~m}$, im Hallstättersee in $30-80 \mathrm{~m}$, im Lunzer Untersee in $20-34 \mathrm{~m}$ Tiefe gefunden. Nach Thí́Baud [1915] aus Schweizer Seen nicht bekannt.

15. Canthocamptus zschokk ei SchMeIL.

Aus dem Litorale des Garschinasees, des Lunzer Obersees, Lunzer Untersees und Partnunersees der Nördlichen Kalkalpenzone bekannt. Bisher fehlen zwischen Lunz und Partnun gelegene Fundorte, ein ähnliches Verhalten wie bei $C$. rhaeticus!

\section{Cyclops.}

Ohne Bezeichnung der Spezies wird das Vorkommen von Cyclops aus folgenden, allen drei Zonen der Ostalpen zugehörigen Fundorten gemeldet: Alpsee bei Füßen, Alpsee bei Immenstadt, Altausseersee, Ammersee, Bannwaldsee, Brennsee, Cavazzosee, Faakersee, Filzsee, Hinterer und Vorderer Finstertalersee, Fuschelsee, Vorderer Gosausee, Gösselsdorfersee, Grundlsee, Grünsee, Hallstättersee, Hechtsee, Hopfensee, Jeserzersee, Keutschachersee, Klopeinersee, Königssee, Königsangersee, Krotensee, Krummsee, Vorderer Langbathsee, St. Leonhardsee, Lichtsee, Loppiosee, Großer und Kleiner Magdalenensee, Mariasteinersee, Millstättersee, Mondsee, Mutterbergersee, Vorderer Oberbergersee, Ödensee, Offensee, Ossiachersee, Plansee, Presseckersee, Saualmsee, Schliersee, Schwarzsee am Schafberg, Seebachsee, Simssee, Sonthofersee, Spitzingsee, Staffelsee, Starnbergersee, Tegernsee, Tihojasee, Traunsee, Vassachersee, Veldersee, Walchensee, Wallersee, Unterer Weißenfelsersee, Wocheinersee, Wolfgangsee, Worstnigsee, Wörthersee und Zellersee.

Hiezu muß hervorgehoben werden, daß die Erwähnung des bloßen Genusnamens Cyclops, auf welche sich die obige Aufzählung gründet, 
meist von älteren Autoren (z. B. Імног) stammt; häufig wurden dann von späteren Beobachtern für einen und den anderen der genannten Fundorte bestimmte Spezies angegeben, worauf bei der Besprechung der einzelnen Arten selbstredend Rücksicht genommen wurde, so daß sich die Nennung einiger Wasserbecken in diesen Fällen wiederholt. Cyclops ist in 14 Spezies und 2 Varietäten aus dem in diesen "Studien“ behandelten Bereiche der Ostalpen bekannt geworden; dazu kommt noch eine unsichere Art (C. magniceps LILLJEBorG). Wenn darunter auch die küsten- und grundbewohnenden wie die hemipelagisch und echt planktonisch lebenden Formen vertreten sind, so darf doch nicht übersehen werden, daß gerade die Planktonuntersuchungen, auf welche die Spezieslisten vieler Seen ausschließlich zurückzuführen sind, uns über das Vorkommen der li t o r a l e n Cyclops-Arten meistens in Unkenntnis gelassen haben. Die Verbreitung der letztgenannten Vertreter wird somit ein noch lückenhafteres Bild liefern als jenes, welches sich derzeit bereits von einzelnen typischen Planktonarten dieses Genus ergibt.

\section{Cyclops affinis G. O. Sars.}

Bisher nur aus dem Litoralgebiet des Unteren Lunzersees (Nördl. Kalkalp.) bekannt.

2. Cy clops a lbidus Jurine (= C. tenuicornis Claus).

Fundorte: Achensee, Faistenauer Hintersee, Niedertrumersee, Partnunersee, Lunzer Obersee und Lunzer Untersee (Nördl. Kalkalp.); Ossiachersee, Piburgersee, Zellersee (Zentralalp.); Idrosee, Iseosee, Großer Magdalenensee (Südl. Kalkalp.).

Auftreten: Vornehmlich küstenbewohnend; im Lunzer Obersee, im Piburgersee und im Partnunersee jedoch auch als Planktonform beobachtet. Diese drei Seen haben geringere Flächenausmaße als die übrigen Fundorte und liegen in Höhen von 915-1874 m ü. d. M.

3. Cyclops bicolor G. O. Sars.

Bisher nur im Lunzer Untersee (Nördl. Kalkalp.) als Litoralbewohner gefunden.

4. $C$ y clo $p s$ d $i$ a phan $u s$ Fischer $(=C$. minutus Claus $=C$. minutus HELLER).

Bekannt aus folgenden Seen der Südlichen Kalkalpenzone: Cavazzosee, Idrosee, Iseosee, San Danielesee und Tovelsee. Angaben über sein Vorkommen in den Zentralalpen- und Nördlichen Kalkalpenseen fehlen. Verläßt im Gegensatze zu den Schweizer Alpen [Thú́baud 1915] nur im Tovelsee (1162 $\mathrm{m}$ ü. d. M.) die Wasserbecken der Talniederungen und Ebene (192-378 m ü. d. M.). 


\section{Cyclops fimbriatus Fischer.}

Fundorte: Attersee, Hallstättersee, Lunzer Mittersee, Lünersee (Nördl. Kalkalp.); Millstättersee (Zentralalp.). Litoralbewohner.

6. $C$ y clo $p s f u s$ c us Jurine $(=C$. coronata Claus $=C$. signatus Косн).

Bisher nachgewiesen im Attersee, Bodensee, Faistenauer Hintersee, Grundlsee, Hallstättersee, Kreuthsee, Lunzer Obersee, Lunzer Untersee, Wildsee bei Seefeld (Nördl. Kalkalp.); Berghaustümpelsee, Lambrechtersee (Zentralalp.); Caldonazzosee, Gardasee, Lavaronesee, Großer Magdalenensee, Großer Montigglersee, Santa Massenzasee, Toblinosee (Südl. Kalkalp.). Für 3 von den genannten 18 Wasserbecken wird ihr pelagisches bezw. hemiplanktonisches Auftreten hervorgehoben (Großer Montigglersee, Lunzer Obersee und Wildsee bei Seefeld), während diese Art sonst als Litoralbewohner genannt ist. Höchstgelegener Fundort: Berghaustümpelsee (1700 m ü. d. M.).

7. Cyclops insignis Claus (=C. quadricornis $\mathrm{KocH})$.

Einziger Fundort: Kalterersee (Südl. Kalkalp.); im Plankton gcfunden, jedoch nicht mit Sicherheit als insignis erkannt!

8. Cyclops leuckarti Claus.

Vorwiegend im Plankton, selten im Litorale folgender Seen nachgewiesen: Bodensee, Chiemsee, Niedertrumersee, Traunsee (Nördl. Kalkalpen); Lansersee, Ossiachersee, Reithersee, Schwarzsee bei Kitzbühel, Zellersee (Zentralalp.); Ceisee, Faakersee, Gardasee, Kalterersee, Keutschachersee, Großer Montigglersee, Weißensee bei Greifenburg, Wörthersee (Südl. Kalkalp.). Gilt als warmwasserliebend und fehlt z. B. im Zellersee während des Winters. Höchstgelegene Fundorte: Ceisee (927 m) und Weißensee $(926 \mathrm{~m})$ ! (Höchstgelegener Fundort in den Schweizer Alpen nach Thí́Baud [1915] Pouillerellacken $1240 \mathrm{~m}$.)

9. Cyclops macrurus G. O. SARS.

Bisher gemeldet aus dem Litoralgebiet folgender Ostalpenseen: Attersee, Faistenauer Hintersee, Krummsee, Niedertrumersee, Simssee, Wallersee (Nördl. Kalkalp.); Ossiachersee, Zellersee (Zentralalp.); Großer Magdalenensee, Großer Montigglersee, Wörthersee (Südl. Kalkalp.). Die Fundorte gehören mit Ausnahme des Krummsees zu den Wasserbecken mit größerer Flächenausdehnung; ihre Höhenlage über dem Meeresspiegel überschreitet in keinem Falle $800 \mathrm{~m}$, da der höchstgelegene Fundort vom Zellersee (749 $\mathrm{m}$ ü. d. M.) repräsentiert wird. Auch aus den Schweizer Alpen nur bis $1040 \mathrm{~m}$ bekannt [Thí́BAud 1915]. 
10. Cyclops magniceps LILLJEBorg.

Diese Cyclops-Spezies wird ausschließlich von Iмноғ [1885] für den Röthelsee im Traunseegebiet (Nördl. Kalkalp.) erwähnt und ist seitdem von keinem Autor mehr wiedergenannt worden; der Fund ist sehr fraglich, die Determination vermutlich ein Irrtum, da die Spezies von G. O. SARs [1913] als Halicyclops magniceps LiLLJBG. für ganz andere Gebiete genannt wird.

11. Cyclops oithonoides G. O. SARs.

Aus dem Plankton des Attersees (Nördl. Kalkalp.) und des Großen Montigglersees (Südl. Kalkalp.) bekannt.

12. Cyclops oithonoides var. hy al in a ReHBERG.

Bisher nur aus dem Ossiachersee (Zentralalp.) -plankton von STEUER [1897] erwähnt.

13. Cyclops serrulatus Fischer (= Leptocyclops agilis [KocH] G. O. SARS 1914).

Fundorte: Attersee, Faistenauer Hintersee, Garschinasee, Grundlsee, Hallstättersee, Kreuthsee, Lunzer Mittersee, Lunzer Obersee, Lunzer Untersee, Lünersee, Niedertrumersee, Pillersee, Unterer Seebisee, Tilisunasee, Traunsee (Nördl. Kalkalp.); Auerlingsee, Anderlesee, Kleiner See südlich von Anderlesee, Filzsee, Fresenhalssee, Oberer Giglachsee, Mittlerer Klippensee, Lambrechtersee, Oberer Lanischsee, Unterer Lanischsee, Lansermoorsee, Millstättersee, Oberer Mühldorfersee, Pfitscherjochseen, Unterer Plenderlesee, Unterer Sonntagskarsee, Weißsee am Tauernjoch, Zellersee (Zentralalp.); Alleghesee, Großer Bödensee, Kleiner Bödensee, Boësee, Caldonazzosee, Ceisee, Kreuzjochsee, Levicosee, Loppiosee, Großer Magdalenensee, Großer Montigglersee, Santa Crocesee, Santa Massenzasee, Toblinosee, Veldessee, Oberer Weißenfelsersee, Wörthersee (Südl. Kalkalp.). - C. serrulatus zählt neben C. strenuus zu den aus dem Ostalpengebiet meist beobachteten Spezies dieser Gattung. C. serrulatus findet sich in allen Arten von Wasserbecken und steigt bis ins Hochgebirge auf, wo er vornehmlich von der litoralen Lebensweise zur planktonischen übergeht; von den aufgezählten Seen liegen 21 über der Waldzone in Regionen von 1930-2333 m ü. d. M.

In Zukunft werden die Beobachter besonders darauf zu achten haben, ob es sich bei alpinen Fundorten tatsächlich überall um diese Spezies und nicht vielleicht um eine der naheverwandten und sehr ähnlichen Formen handelt, die von G. O. SARs [1914] unter den Namen Leptocyclops speratus (LILlJEBorg), L. lilljeborgi G. O. SARS oder vielleicht auch $L$. macruroides (LILLJEBORG) gut beschrieben und abgebildet 
wurden. Vergl. hiezu auch unsere Bemerkungen zur var. denticulata A. Graeter.

14. Cyclops serrulatus var. denticulata A. Graeter.

Bisher nur aus dem Mittersee am Fernpaß (Nördl. Kalkalp.) bekannt geworden. - Ein Vergleich dieser von A. Graeter [1903] zum ersten Male richtig erkannten Varietät mit der von G. O. SARS [1914] als Leptocyclops lilljeborgi aufgestellten Spezies läßt wohl ohne Zweifel die Identität der zwei Formen erkennen. Die Priorität gebührt daher A. Graeter und es bleibt schließlich der subjektiven Ansicht überlassen, die Form als Varietät oder Spezies anzusprechen.

15. Cyclops strenuus Fischer (=C. brevicaudatus Claus $=C$. elongatus $\mathrm{DADAY}=C$. quadricornis LILLJEBORG $=$ C. vicinus UlJanin).

Fundorte: Achensee, Attersee, Bodensee, Chiemsee, Faistenauer Hintersee, Gafiensee. Garschinasee, Grundlsee, Hallstättersee, Hintersee bei Ramsau, Königssee, Leopoldsteinersee, Lunzer Obersee, Lunzer Untersee, Lünersee, Mondsee, Partnunersee, Starnbergersee, Tegernsee, Tilisunasee, Traunsee, Wolfgangsee (Nördl. Kalkalp.); Kleiner See südlich vom Anderlesee, Fresenhalssee, Klammjochsee, Millstättersee. Ossiachersee. Reithersee, Schwarzsee bei Turrach, Sehwarzensee im Zillertal, Unterer Torjochsee, ? Wildsee am Wildseeloder, Zellersee, Zirmsee (Zentralalp.); Großer Bödensee, Caldonazzosee, Ceisee, Faakersee, Gardasee, Lavaronesee, Großer Magdalenensee, Großer und Kleiner Montigglersee, Pragsersee, Santosee, Terlagosee, Tovelsee, Veldessee, Weißensee bei Greifenburg, Wocheinersee, Wörthersee (Südl. Kalkalp.).

Auftreten: Im Plankton des Oberflächenwassers und bis zu $100 \mathrm{~m}$ Tiefe beobachtet, seltener im Litoralgebiet und Seichtwasser; der letzte Fall bezieht sich auf kleinere Wasserbecken des Hochgebirges (Fresenhalssee $2150 \mathrm{~m}$, Unterer Torjochsee $2200 \mathrm{~m}$ ). Die Spezies erscheint somit im Ostalpengebiet horizontal wie vertikal weit verbreitet; die höchstgelegenen Fundorte sind der Schwarzensee im Zillertal (2469 m) und der Zirmsee (2500 $\mathrm{m}$ ü. d. M.). C. strenuus wurde auch im Winterplankton nachgewiesen (z. B. Gardasee und Zellersee).

Es ist bekannt, daß $C$. strenuus Variationen aufweist, die schon von Schmeil [1892] erkannt worden sind, diesen Autor jedoch nicht zur Scheidung in Spezies veranlaßt haben. Wir mußten diesem Standpunkte bei der obigen Aufzählung Rechnung tragen, da es ohne Revision des Materiales natürlich nicht möglich wäre, zu entscheiden, ob und welchem. Fundort vielleicht der bereits von LILLJEBorg [1901] und neuerdings 
von G. O. Sars [1913] erwähnte C. vicinus Uluanin zukommt. Der von Zscнокке [1894] im Gafiensee nachgewiesene C. strenuus wird ausdrücklich als Form ohne pelagische Charaktere bezeichnet, ein Beweis, daß auch in alpinen Seen die beobachteten Variationen vorkommen. Nach den neuesten Untersuchungen von DE LINT [1922], welche sich auf holländisches Material beziehen, wird die Berechtigung der Trennung von $C$. strenuus Fischer und $C$. vicinus UlJanin bestätigt.

16. $C$ y clops vernalis Fischer (= C. elongatus Claus $=C$. lucidulus $\mathrm{KoCH})$.

Bisher wurde diese Spezies in folgenden Ostalpenseen nachgewiesen: Bodensee, Gafiensee, Garschinasee, Lünersee, Partnunersee, Tilisunasee, Zireinersee (Nördl. Kalkalp.); Berghaustümpelsee, Oberer Giglachsee, Kampspitzsee, Oberer Klaftersee, Mittlerer Klippensee, Oberer Mühldorfersee (?), Saureggersee, Schlickersee, Unterer Sonntagskarsee, Speiksee, Weißsee am Tauernjoch (Zentralalp.); aus der Zone der Südlichen Kalkalpen derzeit noch nicht gemeldet. - Es ist auffallend, daß sämtliche der angegebenen Wasserbecken mit Ausnahme des Bodensees in der oberen Waldregion bezw. oberhalb der Waldgrenze in der eigentlichen Hochgebirgsregion gelegen sind; trotzdem handelt es sich bei dieser Cyclops-Spezies sicher nicht um eine stenotherme Kaltwasserform, wie dies früher von Pesta [1911] und auch von Thiébaud [1915, p. 110] vermutet wurde, sondern lediglich um einen widerstandsfähigeren Vertreter des Genus, welcher auch den eigenartigen Daseinsbedingungen im Hochgebirge gewachsen erscheint. Seine Verbreitung außerhalb der Ostalpen und sein Vorkommen in überhitzten Moortümpeln [KLEIBER $1911 ; 22^{\circ} \mathrm{C}$ ] spricht deutlich für die Verschiedenartigkeit der Fundorte.

17. Cyclops viridis JuRINe (=C. brevicornis + gigas Claus, $=C$. clausi HeLler).

Nachgewiesen in folgenden Seen aller drei Ostalpenzonen: Attersee, Bodensee, Faistenauer Hintersee, Hallstättersee, Lunzer Mittersee, Grundlsee (Nördl. Kalkalp.); Lansermoorsee, Millstättersee, Neusiedlersee, Zellersee (Zentralalp.); Alleghesee, Caldonazzosee, Ledrosee, Revinesee, Santa Crocesee, Oberer Weißenfelsersee (Südl. Kalkalp.). - Höchstgelegene Fundorte: Alleghesee $(979 \mathrm{~m})$ und Oberer Weißenfelsersee (940 m ü. d. M.).

Auftreten: Wird meistens unter den Litoral- und Grundbewohnern genannt und soll z. B. im Bodensee erst ab $30 \mathrm{~m}$ Tiefe, im Hallstättersee in 30-80 m Tiefe zu finden sein; seltener als Planktonvertreter erwähnt, wie z. B. im Alleghesee ( $0-30 \mathrm{~m}$ Tiefe) und im Lunzer Mittersee. HoFER 
[1896] zählt die von ihm im Bodensee beobachtete Form zu einer var. caecus!

Zur Verbreitung der Cyclops-Arten vergl. Fig. 14, 15, 16, Taf. VIII.

\section{Diaptomus.}

Ohne Bezeichnung der Spezies für folgende, in diesen "Studien“ einbezogene Wasserbecken (Seen!) nachgewiesen: Alpsee bei Füßen, Alpsee bei Immenstadt, Altausseersee, Ammersee, Attersee, Bannwaldsee, Kleiner See südlich vom Anderlesee, Brennsee, Faakersee, Fuschlsee, Gardasee, Grundlsee, Hallstättersee, Hechtsee, Hopfensee, Jeserzersee, Klopeinersee, Königsee, Krottensee, Vorderer Langbathsee, St. Leonhardsee, Millstättersee, Mondsee, Oberer Mühldorfersee, Nussensee, Offensee, Ossiachersee, Plansee, Schliersee, Schwarzsee bei Kitzbühel, Sonthofersee, Spitzingsee, Staffelsee, Tegernsee, Traunsee, Veldessee, Walchensee, Wallersee, Wolfgangsee, Worstnigsee, Wörthersee, Zellersee und Zireinersee. - Für manche dieser Fundorte haben spätere Beobachter dann die Spezies determiniert, was bei der folgenden Aufzählung berücksichtigt wurde und naturgemäß eine wiederholte Nennung des betreffenden Sees begründet.

Zu den 10 bisher für das Bereich der Ostalpen gemeldeten Spezies (exklusive 1 Varietät) kommt als 11. die von Daday im Neusiedlersee gefundene Form, welche Lokalität als Randsee des Gebietes zum Vergleiche aufgenommen wurde. Die meisten dieser Diaptomus-Arten gehören dem Plankton an und treten hier zeitweise dominierend auf. $\mathrm{Da}$ die Identifizierung in einigen Fällen Schwierigkeiten bereitet, so finden sich in der Literatur mehrfache Berichtigungen früherer $\mathrm{Bc}$ stimmungen, wodurch eine gewisse Unsicherheit bei der Aufzählung der Fundorte entsteht; es schien uns deshalb notwendig, diese Fälle durch genaue Angabe der Zitate festzuhalten. Die Vertreter dieses Genus werden im allgemeinen mit Vorliebe zu tiergeographischen Spekz:lationen herangezogen und spielen bekanntlich auch in der vielumstrittenen Frage der sogenannten Glazialrelikte des Süßwassers eine große Rolle. Wir bekennen uns hier offen zu einem äußerst skeptischen Standpunkt in dieser Beziehung, da die Zwecklosigkeit derartiger Auseinandersetzungen schon vielfach durch den Mangel an Kenntnis einer größeren Anzahl von Fundorten erwiesen worden ist.

Zur Verbreitung einiger Diaptomus-Arten vergl. Fig 17 und 18, Taf. VIII.

1. Diaptomus bacillifer Koelbel. 
Im Plankton folgender Gewässer nachgewiesen: Königssee, Vorderer Langbathsee, Lünersee, Partnunersee, Unterer Seebisee, Tilisunasee (Nördl. Kalkalp.); Kleiner See westlich vom Anderlesee, Oberer Mühldorfersee, Unterer Torjochsee (Zentralalp.); Boësee (Südl. Kalkalp).

Die Angabe des Fundortes Vorderer Langbathsee stammt von Iмноғ [1890, Zool. Anz., vol. 13, p. 655], welcher hier den synonymen D. alpinus fand.

Seit der von Tollinger [1911] gegebenen Zusammenstellung sind für das Gebiet der Ostalpen weitere 5 Fundstellen dieses Diaptomus bekannt geworden; sie reihen sich vollständig entsprechend in das Bild, welches die Verbreitung der Art bisher gezeigt hat; von den hier aufgezählten gesamten 10 Lokalitäten gehören nur 2, nämlich Königssee und Vorderer Langbathsee, den nieder gelegenen Randseen (603 und $675 \mathrm{~m}$ ü. d. M.) an, alle übrigen 8 Wasserbecken fallen in Hochgebirgszonen oberhalb der Waldgrenze (1874-2333 m). Das außeralpine Auftreten und ausgedehnte Vorkommen in der Ebene (z. B. seichte und warme Teiche der Donautiefländer usw.) weist darauf hin, daß D. bacillifer zu den resistenzfähigsten Diaptomus-Arten zählt, so daß von einem stenothermen Kaltwassertier deshalb keine Rede sein kann, wenn auch das Optimum der Verbreitung für Gebirgsländer und in Höhenzonen von 2000-2700 m - nach der Gesamtzahl der bisher gemeldeten Fundorte - errechnet wurde. Holdhaus [1912] hat mit Berechtigung diese Spezies aus seinem kritischen Verzeichnis boreoalpiner Tierformen ausgeschieden.

\section{Diaptomus castor (JURINE).}

Bisher gemeldet aus: Haidersee, Millstättersee (Zentralalp.); Gardasee, Revinesee, Seraiasee (Südl. Kalkalp.). In Seen der Nördlichen Kalkalpenzone nicht nachgewiesen.

Auftreten: Sämtliche Fundorte gehören zu den größeren, im Längenausmaße sogar durchwegs über $1 \mathrm{~km}$ messenden Wasserbecken, von welchen das seichteste noch immer eine Tiefe von 14,6 m (Seraiasee) besitzt; diese Tatsache steht mit den Angaben Tollinger's [1911, p. 6, „D. castor kommt n u r se hr s elt te n in $\mathrm{S}$ e e $\mathrm{n}$ vor . . . Sein Lebenselement sind Kleingewässer, besonders jene, welche regelmäßig im Sommer austrocknen"] in Widerspruch und ist darum hervorzuheben, wenn man nicht etwa annehmen will, daß die Speziesbestimmungen der Autoren unrichtig sind! Höchstgelegene Fundstellen im Gebiete: Seraiasee $(974 \mathrm{~m})$ und Haidersee (1450 m ü.d. M.). Nach Thiś baud 
[1915] in den Schweizer Alpen im Wolfsee (1500) den höchstgelegenen Fundort erreichend.

3. Diaptomus denticornis WIERzeJsKi.

Bisher nur bekannt aus: Garschinasee, Lunzer Obersee (Nördl. Kalkalp.); Lichtsee, Speiksee (Zentralalp.) und Misurinasee (Südl. Kalkalp.)

Lebt in den genannten Wasserbecken durchwegs planktonisch, wenn auch die Fundorte meist nur geringe Tiefen besitzen; die Höhenlagen der letzteren fallen in die Zonen von 1113-2189 m ü. d. M. Stever [1901] führt an, daß er das Vorkommen von D. denticornis in den Salzkammergutseen beobachtet habe, nennt jedoch die betreffenden Gewässer nicht mit Namen.

4. Diaptomus gracilis G. O. SARS.

Fundorte: ? Achensee, Attersee, Bodensee, Chiemsee, Erlaufsee, Faistenauer Hintersee, Grundlsee, Hallstättersee, Königssee, Krottensee, Leopoldsteinersee, Lunzer Untersee, Mondsee, Starnbergersee, Tegernsee, Thumsee, Traunsee, Walchensee, Wolfgangsee (Nördl. Kalkalp.); Auerlingsee, Ossiachersee, Schwarzsee bei Turrach, Zellersee (Zentralalp.); Großer Bödensee, Cavazzosee, Faakersee, Iseosee, Großer Magdalenensee, Wörthersee (Südl. Kalkalp.).

Zum Fundort Achensee ist zu bemerken, daß Вевнu [1902] hier diese Spezies nicht fand be zw. $d$ as Vorkommen des Genus überhaupt in Abrede stellt. Horer [1896] schreibt jedoch „sehr ähnlich wie der Walchensee verhielt sich ferner der Achensee, wo ich am 23. September 1895 auf $85 \mathrm{~m}$ Tiefe nur tote Exemplare von D. gracilis fing, während die ersten lebenden Tiere, hauptsächlich $D$. gracilis und Cyclops strenuus, bei ca. 75 m Tiefe auftauchten"; hiezu meint BREHM [1902]: „Da unmöglich seit 1895 die Zusammensetzung der limnetischen Tierwelt des Achensees eine derartige Änderung erlitten haben kann, glaube ich, daß die Angaben von B. HofER auf eine Materialverwechslung zurückzuführen sind.“ - Eine Revision wäre daher wohl am Platze!, da Faunenänderungen nach unseren Beobachtungen gut möglich sind (siehe Abschnitt I unter Nr. 12 Krummsee!) GarbinI [1895] gibt den Gardasee als Fundort von D. gracilis an; in diesem Falle handelt es sich jedoch um $D$. steueri Brenm et Zederbauer [vergl. Autoren 1904].

Auftreten: Von allen Diaptomus-Arten des Ostalpengebietes am weitesten verbreitet und insbesondere in den größeren Seen der Nördlichen Kalkalpenzone häufig nachgewiesen, wo er im Walchensee $(803 \mathrm{~m})$ und im Erlaufsee (835 m ü. d. M.) vertikal am höchsten emporsteigt, während 
er in den Zentralalpen im Schwarzsee auf der Turracher Höhe schon $1850 \mathrm{~m}$ ü. d. M. erreicht und endlich im Großen Bödensee der Südlichen Kalkalpenzone den höchstgelegenen Standort (2300 m ü. d. M.) besiedelt. D. gracilis wird stets als typischer Planktonvertreter erwähnt, der in Tiefen von $0-35 \mathrm{~m}$ am häufigsten anzutreffen ist.

\section{Diaptomus gra cilis var. carnicus Senna.}

Einziger Fundort: San Danielesee (Südl. Kalkalp.; $270 \mathrm{~m}$ ü. d. M., $700 \mathrm{~m}$ lang, $9 \mathrm{~m}$ tief). Diese Form wird in der Bearbeitung von ToLLinger [1911] nicht genannt; SenNa beschreibt sie 1890. Offenbar handelt es sich um einen analogen Fall wie im Gardasee, wo die ursprünglich als $D$. gracilis determinierte Spezies später als selbständige Art bezw. Varietät ( $D$. steueri) erkannt wurde.

6. Diaptomus graciloides LILLJEBorg.

Bisher nur in 3 Seen des Ostalpengebietes mit Sicherheit nackgewiesen, nämlich im Plankton des Attersee, Simssee (Nördl. Kalkalp.) und Presseckersee (Südl. Kalkalp.). - Als irrtümlich gemeldete Furdorte müssen bezeichnet werden: 1. Wolfgangsee nach KeIssLer [1901]; die Verwechslung mit $D$. gracilis haben Brehm und Zederbauer [1906] festgestellt. 2. Starnbergersee (=Würmsee) nach Zacharias [1905]; die Bestimmung wurde von BREHM [1907] in D. gracilis korrigiert. 3. Gardasee nach Zacharias [1905] von Brehm und Zederbauer [1904] als D. steueri erkannt. - Die Spezies fehlt den Schweizer Alpen [ThiÉbaud 1915].

7. Diaptomus la ciniatus LILLJEBoRg.

Ausschließlich aus dem Attersee, Bodensee und Mondsee (Nördl. Kalkalp.) gemeldet.

8. Diaptomus laticeps G. O. SARS.

Bisher nur im Wocheinersee (Südl. Kalkalp.) nachgewiesen.

9. Diaptomus spinosus Daday.

Hauptbestandteil im Plankton des Neusiedlersees (Randsee an der Zentralalpenzone, des Vergleiches wegen in diese „Studien“ einbezogen). 10. Diaptomus s te u e r $i$ Brehm et Zederbauer.

Bisher nur aus dem Gardasee und Ledrosee (Südl. Kalkalp.) mit Sicherheit nachgewiesen. - Die Form soll im Oberflächenplankton des Gardasees nicht vorkommen, sondern nur in tieferen Wasserschichten gefunden werden.

11. Diaptomus tatricus WierzeJsKi.

Saualmsee (Zentralalp.; $1950 \mathrm{~m}$ ü. d. M.); einziges perennierendes Wasserbecken, welches bisher als Fundort im Ostalpengebiete bekannt 
geworden ist. Dasselbe ist nicht identisch mit dem von Steuer [1897] für diesen Diaptomus genannten Tümpel am Zirbitzkogel, wo (nach einer Berichtigung BrenM's [1915] nicht D. laciniatus, sondern) ebenfalls D. tatricus vorkommt!

12. Diaptomus vulgaris SchMerL.

Bisher nur aus dem Veldessee (Südl. Kalkalp.) bekannt. - Steigt in den Schweizer Alpen nach Thiébaud [1915] bis 1595 m (im Unterstockhornsee).

\section{Heterocope.}

Von diesem Calanidengenus bewohnen 2 Spezies die Seen des Ostalpenbereiches, wo sie stets an der Zusammensetzung des Planktons teilhaben. Da die Unterscheidung der Arten mehrmals mißlungen war und zu irtümlichen Angaben Veranlassung gab, wurde auf eine kritische Prüfung der Fundorte besonderes Augenmerk gerichtet.

1. Heterocope saliens LiLlJeborg.

Diese Spezies wurde mit Sicherheit für folgende Wasserbecken nachgewiesen: Augstwiesensee, Mittersee am Schafberg, Traunsee, Wildsee bei Seefeld (Nördl. Kalkalp.); Anderlesee, Kleiner See südlich rom Anderlesee, Kleiner See westlich vom Anderlesee, Fresenhalssee, Schwarzsee bei Turrachhöhe (Zentralalp.).

Den Fundort Mittersee am Schafberg (Salzkammergut, $1334 \mathrm{~m}$ ü. d. M., $100 \mathrm{~m}$ im Durchmesser, Maximaltiefe 7,6 m) erwähnt LangHavs [1908] in seiner Abhandlung über „Das Plankton des Traunsees in Oberösterreich", p. 211, vorletzter Absatz, weshalb er in der bisher erschienenen Literatur stets übersehen und von uns auch erst nach Abschluß des Manuskriptes zum vorigen Abschnitt dieser "Studien“ entdeckt wurde. Nach Tollinger [1911] soll H. saliens ferner auch im Chiemsee und Iseosee vorkommen; die irrtümliche Nennung des ersteren Sees wurde inzwischen von mehrfacher Seite richtig gestellt (vergl. hiezu unsere Angaben sub Nr. 25 Chiemsee und unten bei H. weismanni); bezüglich des Iseosee müssen wir bezweifeln, ob Brehm und Zederbauer [1906] mit der Äußerung „Der von H. saliens bewohnte Südgürtel . . . dürfte mit dem Spinone- und Iseosee im Osten enden" auch das tatsächliche Vorkommen dieser Form im Iseosee gemeint ist. Pavesi [1879] erwähnt sie nicht.

Mit Ausnahme des Traunsees zählen alle von uns genannten Fundorte zu kleinen, seichten oder nur einige Meter tiefen Wasserbecken, von welchen die eine Hälfte noch in der Waldregion (1180-1850 m), die andere in der waldlosen Hochgebirgsregion $(2000-2150 \mathrm{~m})$ liegt. 
2. Heterocope weismanni. Імноғ.

Bisher ausschließlich im Plankton folgender größerer Seen am Nordrande der Nördlichen Kalkalpenzone nachgewiesen: Bodensee, Chiemsee, Schliersee, Starnbergersee. - Die Form wurde an der Oberfläche und bis in Tiefen von $60 \mathrm{~m}$ angetroffen. Das Vorkommen von H. weismanni (nicht saliens!) im Chiemsee wurde von den Autoren Brehm [1906], Haempel [1913] und Baumbach [1922] einwandfrei festgestellt.

Da die allgemeine geographische Verbreitung von $H$. weismanni pine "Auslöschungszone" im Sinne der Ausführungen von HoldHaus. [1912] zeigt, erscheint sie in das kritische Verzeichnis der boreoalpinen Tierformen (Glazialrelikte) des genannten Autors aufgenommen. Auffällig bleibt der bisher mangelnde Nachweis in größeren und tieferen Seen der Zentralalpenzone, wie auch in der eigentlichen Hochgebirgsregion im allgemeinen. Zur Verbreitung der Heterocope-Arten vergl. Fig. 19, Taf. VIII.

\section{Moraria.}

Unsere Kenntnisse über das Vorkommen der verschiedenen Harpacticidengenera in Seen sind derzeit noch äußerst lückenhaft; gilt dies schon für die länger bekannte Gattung Canthocamptus, dann um so mehr für andere Mitglieder der Familie. Aus dem Genus Moraria gelang es erst in allerletzter Zeit, einen Vertreter im Bereiche der Ostalpenseen aufzufinden.

Moraria schmeili van Douwe.

Bisher bekannt aus dem Erlaufsee, Lunzer Obersee und Lunzer Untersee (Nördl. Kalkalp.). - Die Fundorte liegen in geringen Entfernungen voneinander, in vertikalen Höhen von 608-1113 m ü. d. M.

\section{Cladocera.}

Acroperus.

Infolge der Lücken, welche über den Grad der Variation dieses Genus unseren derzeitigen Kenntnissen noch immer anhaften, gehen die Ansichten über die Unterscheidung der hierher gehörigen Formen ziemlich auseinander; wenn daher ältere Autoren meist nur Acroperus harpae BAIRD (=A. leucocephalus KocH) aufzuzählen haben, so bedeutet dies mehr oder weniger einen Sammelnamen, welcher natürlich nicht erlaubt, irgendeine Entscheidung darüber zu treffen, ob die typische oder eine variante Form gemeint war. Dies ist bei der folgenden Fundortsliste 
von $A$. harpae zu beachten. Wo jedoch die vorgefundene Angabe bereits genauer gefaßt war, haben wir uns einer ebensolchen Unterscheidung angeschlossen und eventuell nur die subjektive Verschiebung von einer Varietät zu einer selbständigen Spezies vorgenommen; dies gilt für die Trennung des $A$. angustatus G. O. SARS von $A$. harpae BaIRD. Gewissenhafte Untersuchungen über die Merkmale der zwei genannten Spezies stammen von STINGELin [1906].

Die Acroperus-Formen sind zweifellos Litoralbewohner; wenn ihr Auftreten im Plankton auch ganz richtig beobachtet worden ist, so kann es sich in diesen Fällen doch nur um zufällige Gelegenheitserscheinungen handeln, die ihre Ursache in stärkeren Strömungen oder im Aufwühlen der obersten Bodenschichten oder Abtrieb des Algenbelages haben mögen, was bei seichteren Wasserbecken leicht eintreten kann. Ohne Bezeichnung der Spezies wird das Vorkommen der Gattung nur für den Königsangersee (Zentralalp.; $2173 \mathrm{~m}$ ü. d. M.) genannt.

1. A croperus angustatus G. O. SARS.

Es wird diese Spezies vielfach als $A$. harpae var. angustatus aufgeführt. Ihr Vorkommen ist mit Sicherheit für folgende Fundorte nachgewiesen: Attersee, Grundlsee, Hallstättersee, Hintersee bei Ramsau, Königssee, Lunzer Untersee (Nördl. Kalkalp.); Ceisee, Loppiosee, Großer Montigglersee, Santosee, Veldessee (Südl. Kalkalp.); Zellersee (Zentralalp.). Höchstgelegener Fundort: Ceisee (927 m ü. d. M.).

Demnach würde diese Form die Zone der Nördlichen und Südlichen Kalkalpen nur im Zellersee verlassen und außerdem der Fauna höher gelegener Gebirgs- und Hochgebirgsseen fehlen. Das erstgenannte Verhalten sagt uns derzeit mit Rücksicht auf das fragliche Erkennen der Spezies nichts Sicheres; hingegen würde ihr Fehlen im Hochgebirge mit den Beobachtungen in den Westalpen (Schweiz) übereinstimmen, denn das Vorkommen in dem $2453 \mathrm{~m}$ ü. d. M. gelegenen Lago scuro (Tessin) wird von Stingelin [1908] als fraglich bezeichnet.

Auftreten: Litoral.

2. Acroperus harpa e BAIRD (=A. leucocephalus autorum).

Man vergleiche hiezu unsere Bemerkungen bei Aufzählung des Genus.

Fundorte: Attersee, Augstsee, Berglsteinersee, Bodensee, Faistenauer Hintersee, Garschinasee, Grundlsee, Hallstättersee, Igelsee. Kammersee, Hinterer Lahngangsee, Lunzer Mittersee, Lunzer Obersee und Lunzer Untersee, Mariasteinersee, Mittersee am Fernpaß, Niedertrumersee, Nussensee, Vorderer Oberbergersee, Ödensee, Partnunersee, Reintalersee, Sommersbergersee, Tilisunasee, Traunsee, Zireinersee (Nördl. Kalkalp.); 
Anderlesee, Filzsee, Gaishornsee, Oberer Giglachsee, Grünsee, Mittlerer Klippensee, St. Leonhardsee, Unterer Mühldorfersee, Ossiachersee, Oberer Sonntagskarsee, Unterer Sonntagskarsee, Turrachersee, Millstättersee, Zellersee (Zentralalp.); Gösselsdorfersee, Klopeinersee, Großer Magdalenensee, Tihojasee, Wocheinersee, Wörthersee (Südl. Kalkalp.).

Höchstgelegene Wasserbecken: Mittlerer Klippensee (2257 m) und Unterer Mühldorfersee (2281 m ü. d. MI.); daran schließen sich weitere acht oberhalb der Waldregion befindliche Seen. Der Gegensatz in der vertikalen Ausbreitung im Vergleiche zu $A$. angustatus entspricht dem Vorkommen in den Westalpen, wo die Art noch in einem $2610 \mathrm{~m}$ ü. d. M. gelegenen See im St. Bernhardmassiv nachgewiesen wurde [STINGELIN 1908].

Auftreten: Von der Mehrzahl der Beobachter als Litoral- oder Grundbewohner gemeldet, soll sie im Lunzer Mittersee und Lunzer Obersee als Pianktonvertreter vorkommen; es dürfte das abweichende Verhalten kaum eine normale, d. h. konstante Erscheinung darstellen. 3. Acroperus harpae var. trigida Ekman.

Bisher nur für den Kammersee (Nördl. Kalkalp.) bekannt geworden.

\section{Alona (= Lynceus autorum).}

Dieses für die Litoralbezirke stehender Gewässer sehr typische Genus ist im Bereiche der hier einbezogenen Ostalpenseen mit 6 Spezies vertreten; die von manchen Autoren als Varietät verzeichnete affinisForm wird dabei von uns, gleich vielen anderen Vorgängern, zu einer selbständigen Art gezählt. Ob sich unter dem Namen Alona quadrangularis in einzelnen Fällen nicht auch affinis verbirgt, kann nicht mit Sicherheit entschieden werden. A. (Lynceus) rostrata (KocH) fällt unter Rhynchoalona.

Das Vorkommen des Genus ohne nähere Bezeichnung der Spezies ist gemeldet für den Königsangersee und ? Unteren Lanischsee (Zentralalpen).

1. Alona affinis (LeYdig) (=A. quadrangularis var. affinis = Lynceus affinis).

Fundorte: Achensee, Attersee, Augstsee, Augstwiesensee, Faistenauer Hintersee, Hallstättersee, Kammersee, Kreuthsee, Vorderer Lahngangsee, Lunzer Mittersee, Münichsee, Nassereithersee, Vorderer Oberbergersee, Ödensee, Schwarzsee am Schafberg, Schwarzer See beim Ödensee, Unterer Seebisee, Sommersbergersee, Traunsee, Zireinersee (Nördl. Kalkalp.); Anderlesee, Kleiner See südlich vom Anderlesee, Kleiner 
See westlich rom Anderlesee, Berghaustümpelsee, Oberer Giglachsee, Grünsee, Haidersee, Oberer Klaftersee, Oberer Mühldorfersee, Unterer Mühldorfersee, Mutterbergersee, Ossiachersee, Saualmsee, Saureggersee, Schlickersee, Sch warzsee bei Turrach, Unterer Sonntagskarsee, Turrachersee (Zentralalp.); Großer Bödensee, Kleiner Bödensee, Kreuzjochsee, Großer Montigglersee, Tovelsee, Wörthersee (Südl. Kalkalp.).

Hier ist beizufügen, daß die von STINGELIN beschriebene ornataForm, welche wir nicht als Varietät betrachten, im Oberen Giglachsee, im Oberen Klaftersee, im Kreuzjochsee und im Unteren Sonntagskarsee von uns wiedergefunden wurde. Nach den bisherigen Angaben zu schließen, hat $A$. affinis sowohl in horizontaler wie auch in vertikaler Richtung die weiteste Verbreitung im Bereiche der Ostalpen unter allen Alona-Arten.

Höchstgelegene Wasserbecken: Mutterbergersee $(2483 \mathrm{~m})$ im Gebiet der Stubaier Alpen (Zentralalp.) und Bödenseen $(2300 \mathrm{~m})$ in den Südtiroler Dolomiten (Südl. Kalkalp.).

2. Alona costata G. O. SARS.

Bisher nachgewiesen im: Attersee, Augstsee, Bodensee, Grundlsee, Hallstättersee, Hinterer Lahngangsee, Lunzer Untersee, Mittersee am FernpaB, Ödensee, Partnunersee, Sommersbergersee, Traunsee (Nördl. Kalkalp.); Gaishornsee, Reithersee (Zentralalp.); Jeserzersee, Großer Magdalenensee, Veldessee, Wörthersee (Südl. Kalkalp.).

Auftreten: Lebt im Litorale sehr verschiedenartiger Seen aller drei Zonen und in allen Höhenlagen; höchster Fundort im Ostalpengebiet bisher: Partnunersee (1874 m).

3. Alona gut tata G. O. SARS.

Fundorte: Attersee, Augstsee, Berglsteinersee, Grundlsee, Hallstättersee, Lunzer Obersee, Lunzer Untersee, Ödensee, Sommersbergersee, Traunsee, Wildensee (Nördl. Kalkalp.); Jeserzersee, Großer Magdalenensee, Großer Montigglersee (Südl. Kalkalp.).

Diese Spezies ist bisher im Zentralalpengebiet noch nicht gefunden worden. Aus dem Ödensee wird neben dem Vorkommen der Type auch die als Variation (?) bezeichnete Form tuberculata ThIÉBaUd gemeldet. Höchstgelegene Fundstelle: Wildensee $(1554 \mathrm{~m})$.

4. Alona quadrangularis (0. F. MüLler).

Wird für folgende Ostalpenseen genannt: Attersee, Augstsee, Faistenauer Hintersee, Garschinasee, Grundlsee, Hallstättersee, Kammersee, Kröllensee, Münichsee, Niedertrumersee, Sommersbergersee, Wallersee (Nördl. Kalkalp.); Auerlingsee, Lambrechtersee, Millstättersee, 
Unterer Sonntagskarsee, Vassachersee (Zentralalp.); Caldonazzosee, Gardasee, Lagoraisee, Levicosee, Großer Magdalenensee, Großer Montigglersee, Seraiasee, Oberer Weißenfelsersee (Südl. Kalkalp.).

Vergleiche hiezu unsere Bemerkungen sub Genus Alona. - Auftreten: Litoral. Höchstgelegene Wasserbecken: Garschinasee (2189 m), Unterer Sonntagskarsee $(1930 \mathrm{~m})$ und Lagoraisee $(1868 \mathrm{~m})$.

5. Alona rectangula G. O. SARS.

Fundorte: Attersee, Augstsee, Augstwiesensee, Bodensee, Grundlsee, Niedertrumersee, Wallersee (Nördl. Kalkalp.); Leonhardsee, Zellersee (Zentralalp.); Kreuzjochsee, Loppiosee, Tihojasee, Veldessee (Südl. Kalkalp.).

Im Niedertrumersee auch die var. pulchra Matile (neben der typischen Form) angeführt.

Höchstgelegener Fundort: Kreuzjochsee (2200 m ü. d. M.). Auftreten: Im Litorale.

6. Alona tenuicaudis G. O. SARS.

Bisher nur aus dem Veldessee (Südl. Kalkalp.; 478 m ü.d. M.) bekannt. Zur Verbreitung einiger Alona-Arten vergl. Fig. 20 Taf. VIII.

\section{Alonella.}

Das Vorkommen des Genus (ohne Bezeichnung der Spezies) wird aus dem Millstättersee gemeldet. Aus dem Ostalpengebiet in 3 Arten bekannt. Die Formen gehören zur Litoralfauna, einzelne Beobachtungen vom Auftreten im Plankton dürften kaum auf normalen und konstanten Erscheinungen beruhen.

1. Alonella excisa (Fischer) (= Pleuroxus excisus autorum).

Fundorte: Achensee, Faistenauer Hintersee, Niedertrumersee, Partnunersee (Nördl. Kalkalp.); Berghaustümpelsee, Oberer Klaftersee, Klammjochsee, Lansermoorsee (Zentralalp.); Ceisee, Kreuzjochsee, Großer Montigglersee (Südl. Kalkalp.).

Die Spezies steigt demnach in allen drei Zonen im Hochgebirge auf. Nach Stingelin [1908] in der Schweiz noch in Höhen von $2620 \mathrm{~m}$ nachgewiesen.

2. A l o n ell a e xigua (LilljeboRg).

Bisher nur aus dem Litorale von drei großen Talseen bekannt, nämlich: Niedertrumersee, Wallersee (Nördl. Kalkalp.) und Zellersee (Zentralalp.).

3. A lo nell a n a n a BAIRD).

Fundorte: Ammersee, Hintersteinersee, Mariasteinersee, Niedertrumersee, Lunzer Obersee, Lunzer Untersee, Wallersee (Nördl. Kalkalp.); 
Kampspitzsee, Schwarzsee bei Kitzbühel (Zentralalp.); Ceisee, Terlagosee (Südl. Kalkalp.).

Höchstgelegener See: Kampspitzsee (2200 m ü. d. M.) und Lunzer Obersee $(1113 \mathrm{~m})$. A. nana wurde im Ammersee und in den beiden Lunzerseen auch als Planktonbestandteil gefangen; bezüglich des ersteren Falles hebt der Autor [Baumbach 1922] hervor, daß es nicht wahrscheinlich sei, diese Cladocere unter gewöhnlichen Umständen als eulimnetisches Tier im Seeplankton anzutreffen.

\section{Alonopsis.}

Alonopsis elongata G. O. SARS.

Fundorte: Achensee, Attersee, Bodensee, Faistenauer Hintersee, Grundlsee, Niedertrumersee, Pillersee, Wallersee (Nördl. Kalkalp.).

Nach den bisherigen Kenntnissen über die Verbreitung im Ostalpengebiet würde sich Alonopsis elongata somit auf die größeren Seen in niederen Höhenlagen (465-930 m ü. d. MI.) beschränken; sie erscheint hier durchwegs als Litoralbewohner, auch in der Schlammfazies lebend. Auffällig ist auch ihr ausschlieBlicher Nachweis in Seen der Nördlichen Kalkalpenzone; wenn nicht neuere Funde diesbezüglich andere Lokalitäten zur Kenntnis bringen, so stimmt dieses Verhalten mit der Lage jener Fundorte überein, welche Stingelin [1908] für die Schweizer Alpen aufzählt. Vergl. Fig. 22, Taf. VIII.

\section{Bosmina.}

Da die Einreihung der zahlreichen zu diesem Genus gehörigen Formen noch vielfach unsicher ist und ganz subjektiven Entscheidungen unterliegt, sind in der folgenden Aufzählung vornehmlich die Angaben über das Vorkommen der beiden von BURcKHARDt unterschiedenen Hauptarten, Bosmina coregoni BAIRD und B. longirostris (O. F. MÜLLER), in Betracht zu ziehen; im übrigen wurde die Zuweisung der für die Ostalpenseen nachgewiesenen Bosminen nach der Bearbeitung von KeILHACK [1909] vorgenommen, alle zweifelhaften Fälle außerdem vermerkt. Die Benennung durch ältere Autoren kann überdies auch aus unseren Listen bei den im vorhergehenden Kapitel besprochenen Fundorten entnommen und verglichen werden.

Ohne Bezeichnung der Spezies wurde das Vorkommen von Bosmina für folgende Ostalpenseen erwähnt: Alpsee bei Immenstadt, Altaussersee, Attersee, Bannwaldsee, Fuschlsee, Vorderer Gosausee, Grundlsee, Hallstättersee, Hechtsee, Hopfensee, Königssee, Krottensee, Vorderer Lang- 
bathsee, Mondsee, Vorderer Oberbergersee, Ödensee, Offensee, Plansee, Schliersee, Schwansee, Schwarzsee ani Schafberg, Spitzingsee, Staffelsee, Starnbergersee, Tegernsee, Walchensee, Wallersee, Weißensee bei Füssen, Wolfgangsee (Nördl. Kalkalp.); Brennsee, Leonhardsee, Ossiachersee, Reithersee, Oberer Sonntagskarsee, Vassachersee, Zellersee (Zentralalp.); Faakersee, Jeserzsee, Keutschachersee, Klopeinersee, Großer und Kleiner Magdalenensee, Raiblersee, Seebachsee, Veldessee, Wocheinersee, Wörthersee (Südl. Kalkalp.).

Für einige der hier aufgezählten Seen fand eine Determination der betreffenden Bosminen durch Untersuchungen seitens späterer Beobachter statt, worauf bei der Besprechung der im folgenden genannten Formen Rücksicht genommen wurde, so daß in diesen Fällen die Nennung des Fundorts wiederholt scheint.

Sämtliche Bosminen bilden einen charakteristischen Bestandteil im Plankton der genannten Seen. Die große Zahl (47) der Fundorte spricht für die weite Verbreitung des Genus im Ostalpengebiet; sie wird sich nach weiter fortgesetzten Bearbeitungen noch wesentlich erhöhen.

1. Bosmina coregoni BAIRD [BURCKHARDT].

Fundorte: Achensee, Altausseersee, Attersee, Chiemsee, Erlaufsee, Hintersee bei Ramsau, Leopoldsteinersee, Lunzer Mittersee, Lunzer Obersee, Mondsee, Traunsee, Wolfgangsee (Nördl. Kalkalp.); Ossiachersee (Zentralalp.); Großer Magdalenensee, Wocheinersee, Wörthersee (Südl. Kalkalp.).

Für den Großen Magdalenensee, Ossiachersee, Wocheinersee und Wörthersee wird speziell die Form B. longicornis SchoedLer genannt, die im letzten See auch neben der Type vorkommen soll. - Die Form aus dem Achensee will Brenm [1902] in nächster Nähe der dollfusiceresiana-Gruppe stellen, ZaCHARIAS [1903] jedoch zur var. lariana BURCKH. rechnen.

Auftreten: Planktonisch an der Oberfläche und bis in $100 \mathrm{~m}$ Tiefe; im Achensee perennierend mit zwei Maxima der Entwicklung, nämlich im März und Oktober-November. - Höchstgelegener Fundort: Lunzer Obersee (1113 m ü. d. M.).

Bosmina coregoni-longispina LeYdig.

Fundorte: Ammersee, Attersee, Bodensee, Grundlsee, Hallstättersee, Königssee, Vorderer Lahngangsee, Lunzer Untersee, ? Starnbergersee, Traunsee, Wolfgangsee (Nördl. Kalkalp.); Oberer Giglachsee, Haidersee, Ossiachersee (Zentralalp.); Cavazzosee, Faakersee, Kalterersee, Keut- 
schachersee, Ledrosee, Seraiasee, Weißensee bei Greifenburg (Südl. Kalkalp.).

Speziell als forma bohemica Stixgelin für den Faakersee, Keutschachersee, Lunzer Untersee, Ossiachersee (neben der Type!), ? Starnbergersee, Weißensee bei Greifenburg und Wolfgangsee genannt.

Höchstgelegener Fundort: Oberer Giglachsee (1960 m ü. d. M.).

Als forma dollfusi Moniez speziell für den Haidersee und als forma kessleri UlJanin für den Ammersee gemeldet.

Bosmina coregoni var. a crocoregoni (BURCKHARDT).

Bisher nur aus dem Simssee (Nördl. Kalkalp.), und zwar als forma burckhardti nachgewiesen.

Bosmina coregoni var. a methystina BREHM.

Einziger, bisher bekannter Fundort: Gardasee (Südl. Kalkalp.).

2. Bosmina longirostris (O. F. Müller) [BurckhardT]. Fundorte: Ammersee, Attersee, Bodensee, Chiemsee, Erlaufsee, Rissersee, Sommersbergersee, Thumsee, Tiersee, Traunsee, Wallersee (Nördl. Kalkalp.); Lauterersee, Ossiachersee, Piburgersee, Schwarzsee bei Kitzbühel, Zellersee (Zentralalp.); Caldonazzosee, Cavazzosee, Ceisee, Kalterersee, Lasessee, Lavaronesee, Levicosee, Loppiosee, Großer und Kleiner Montigglersee, Piazzesee, Pragsersee, Presseckersee, San Danielesee, Santa Massenzasee, Santosee, Serajasee, Terlagosee, Toblinosee, Veldessee, Weißensee bei Greifenburg (Südl. Kalkalp.). - Im Attersee wurde die forma pelagica Strngelin nachgewiesen. Die forma brexicornis Hellich wird speziell für den Ammersee und Wallersee genannt, die forma cornuta JuRINe für folgende Seen: Bodensee, Kalterersee, Lavaronesee, Loppiosee, Großer und Kleiner Montigglersee, Piazzesee, Pragsersee, Rissersee, Schwarzsee bei Kitzbühel, Serajasee, Sommersbergersee, Terlagosee, Traunsee, Veldessee; letztere wurde in vielen Fällen neben der typischen longirostris-Form vorkommend beobachtet. Man vergleiche hiezu unsere Verbreitungskarte! Fig. 21, Taf. VIII.

Auftreten: Im Zellersee fehlt B. longirostris während des Winters; sie wird für alle Seen als Planktonvertreter angegeben, nur im Veldessee soll sie außerdem auch als Litoralbewohner auftreten.

Höchstgelegene Fundorte sind: Lauterersee (2400 m) und Pragsersee (1496 m ü. d. M.).

Nach unseren bisherigen Kenntnissen sind somit beide Hauptarten von Bosmina (B. coregoni und B. longirostris) in allen Zonen des Ostalpengebietes verbreitet, kommen mehrmals in einem Fundort zugleich vor und steigen in die waldlose Hochgebirgsregion empor. 


\section{Bythotrephes,}

Das Genus wird nur durch eine Spezies vertreten, nämlich Bythotrephes longimanus LEYDIG.

Nachgewiesen im: Altaussersee, Ammersee, Attersee, Bodensee, Chiemsee, Grundlsee, Hallstättersee, Krottensee, Vorderer Lahngangsee, Vorderer Langbathsee, Mondsee, Plansee, Tegernsee, Toplitzsee, Traunsee, Wolfgangsee (Nördl. Kalkalp.); Millstättersee, Zellersee (Zentralalp.); Gardasee, Iseosee (Südl. Kalkalp.).

In dieser Zusammenstellung der Fundorte findet die schon erkannte Tatsache Bestätigung, daß Bythotrephes longimanus eine der wenigen Cladoceren ist, die in seichten Wasserbecken stets fehlt; der Vordere Langbathsee mit 33,8 m Tiefe repräsentiert in der Liste den Wohnort von geringster Tiefe. Alle übrigen Fundstellen gehören fast ausnahmslos zu den tieferen und größeren Randseen im Norden und Süden des Ostalpenzuges oder liegen als große Talseen innerhalb dieses Gürtels (Millstättersee und Zellersee); als bemerkenswerte Ausnahme erscheint der Krottensee am Schafberg, der dem Vorkommen der Spezies zwar in bezug auf die Tiefenverhältnisse entspricht $(45,5 \mathrm{~m})$, jedoch ein sehr geringes Flächenausmaß besitzt (390 $\mathrm{m}$ im Durchmesser).

Im Vorderen Lahngangsee (1555 m ü. d. M.) erreicht das Vorkommen von B. $l$. den höchstgelegenen Punkt, der unseres Wissens für die Alpen überhaupt bekannt ist, da Stingelin [1908] für die Schweizer Alpen den Lac de Joux in $1008 \mathrm{~m}$ ü. d. M. als höchste Lokalität in vertikaler Erhebung bezeichnet.

Auftreten: Obwohl B. l. zu wiederholten Malen auch aus dem Oberflächenplankton gemeldet wurde, genießt diese Cladocere doch im besonderen den Ruf eines Bewohners der Tiefenregionen, wobei auf das mächtig entwickelte Auge hingewiesen wird. Es soll deshalb an dieser Stelle erwähnt werden, daß eine naheverwandte Cladocere, nämlich Polyphemus pediculus (LrNNÉ), ebenso auffallend große Augen besitzt, jedoch gerade in größeren Seen häufig litoral lebt, außerdem aber vielfach in ganz seichten, tümpelartigen Gewässern nachgewiesen wurde. Somit scheint uns eine direkte Beziehung zwischen der Größe des Sehorganes und dem Aufenthalt in tieferen Wasserschichten nicht bestehen zu müssen (vergl. darüber auch die Abhandlung von S. EкмaN [1904].

B. longimanus gilt als monozyklisch und soll im Winterplankton stets fehlen (z. B. Hallstättersee und Zellersee). Zur Verbreitung im Gebiete vergl. Fig. 22, Taf. VIII. 


\section{Camptocercus.}

Aus den Seen der Ostalpen wurde dieses Genus bisher in 2 Spezies und 1 Varietät bekannt. Im Hochgebirge nicht vertreten.

1. Camptocercus lilljeborgi SchoEdLER.

Bisher nur aus dem Bodensee und Igelsee (Nördl. Kalkalp.) gemeldet.

2. Camptocercus rectirostris (Schoedler).

Nachgewiesen im: Niedertrumersee, Traunsee (Nördl. Kalkalp.); Ceisee, Jeserzersee, Klopeinersee, Großer Magdalenensee (Südl. Kalkalp.). - Aus einem See der Zentralalpenzone bisher nicht bekannt.

3. Camptocercus rectirostris var. biserratus (SchoedLeR).

Ausschließlich aus dem Igelsee (Nördl. Kalkalp.) gemeldet, wo auch C. Tilljeborgi vorkommt.

\section{Ceriodaphnia.}

Die Arten der Gattung Ceriodaphnia bilden teils charakteristische Planktonkomponenten, teils ausgesprochene Litoralbewohner; im Gebiete der Ostalpen wurden bisher aus Seen 5 verschiedene Spezies und 1 Varietät bekannt. Fehlt im Hochgebirge.

Ohne nähere Bezeichnung der Art wird das Vorkommen von Ceriodaphnia aus folgenden Fundorten gemeldet: Gösselsdorfersee, Jeserzsee, Kentschachersee, Klopeinersee, Großer Magdalenensee, Presseckersee, Seebachsee, Worstnigsee (Südl. Kalkalp.); Lansersee, Ossiachersee, Vassachersee, Zellersee (Zentralalp.).

1. Ceriodaphnia la tica ud a ta P. E. MÜLLER.

Bisher im Krottensee und Lunzer Untersee (Nördl. Kalkalp.) nachgewiesen.

2. Ceriodaphnia megops G. O. SARS.

Nur aus dem Gaishornsee (Zentralalp.) bekannt.

3. Ceriodaphnia pulchella G. O. SARS.

Fundorte: Hallstättersee, Igelsee, Kammersee, Krummsee, Münichsee, Niedertrumersee, Rissersee, Sommersbergersee (Nördl. Kalkalp.); Ceisee, Loppiosee, Großer und Kleiner Montigglersee, Santosee, Veldessee, Weißensee bei Greifenburg, Wörthersee (Südl. Kalkalp.); Schwarzsee bei Kitzbühel, Millstättersee, Zellersee (Zentralalp.).

Auftreten: Planktonisch (z. B. Montigglersee, Veldessee) oder litoral (z. B. Hallstättersee, Millstättersee, Niedertrumersee, Zellersee).

4. C eriodaphnia quadrangula (O. F. MüLLER).

Im Gegensatze zur später angeführten var. hamata aus allen drei Ostalpenzonen bekannt, nämlich aus dem: Bodensee, Thumsee (Nördl. Kalkalp.); Cavazzosee, Iseosee, Loppiosee, Revinesee, Santa Massenzasee, 
Toblinosee, Weißensee bei Greifenburg (Südl. Kalkalp.); Piburgersee (Zentralalp.).

Lebt im Bodensee als Litoralbewohner, im Piburgersee und Thun see planktonisch.

5. Cerioda phnia quadrangul a var. h a ma t a LiLLJEBorg.

Bisher nur in einigen Seen der Nördlichen Kalkalpenzone nachgewiesen: Igelsee, Königssee, Münichsee, Tiersee. - Für den Königssee und Tiersee als Pianktonform bezeichnet.

6. Ceriodaphnia reticulata (JURINE).

Fundorte: Krottensee und Wörthersee.

Zur Verbreitung von C. pulchella im Gebiete vergl. Fig. 22, Taf. VIII.

\section{Chydorus.}

Nur 1 Spezies dieser Gattung erfreut sich einer überraschend weiten Verbreitung, nämlich Chydorus sphaericus, während die übrigen Arten im Gegensatze dazu verschwindend selten angegeben werden; es sind deren $4 \mathrm{im}$ Ostalpengebiet noch nachgewiesen worden. Mit Rücksicht auf dieses Mißverhältnis in der Zahl der Fundorte erscheint die Vermutung berechtigt, daß sich unter dem vielgenannten $C h$. sphaericus: eine Art „Sammelname“ verbirgt, der in zahlreichen Fällen einer kritischen Nachprüfung bedürfen würde; es soll deswegen nicht geleugnet werden, daß Ch. sphaericus tatsächlich zu den häufigsten Cladoceren aller Arten von Seen gehört, wie diese Form ja überhaupt als Kosmopolit und Ubiquist im weitesten Sinne bekannt ist.

Die Chydorus-Arten sind zweifellos typische Litoraltiere. Nichtsdestoweniger liegen zahlreiche Beobachtungen vor, welche das planktonische Auftreten betonen und speziell für die kleineren Seen im Hochgebirge namhaft machen. Auch das Vorkommen in der Schlammfazies selbst, sowie in größeren Tiefen $(80 \mathrm{~m})$ wurde nachgewiesen. Die Tiere vertragen in jeder Beziehung die verschiedensten und extremsten Milieubedingungen.

Als Fundorte des Genus (ohne Angabe der Art) seien der Vollständigkeit halber hier noch angeführt: Filzsee, Jeserzersee, Königssee und Königsangersee.

1. Chydorus gibbus LILLJEBoRG.

Bisher nur im Litorale des Attersee, Niedertrumersee und Zellersee nachgewiesen.

2. Chy dorus globosus BAIRd. 
Ebenfalls nur aus dem Attersee, Grundlsee und Niedertrumersee (Nördl. Kalkalp.) bekannt.

3. Chydorus latus G. O. SARS.

Bisher nur aus dem Attersee (Nördl. Kalkalp.) und Keutschachersee, Großer Magdalenensee (Südl. Kalkalp.) gemeldet.

4. Chydorus piger G. O. SARS.

Nur aus dem? Achensee (Nördl. Kalkalp.) und Tschampeisee (Südl. Kalkalp.) bekannt. - Das Vorkommen im Achensee wurde von ScourFIELD (unter dem Synonym Ch. barbata) erwähnt, konnte jedoch von BREHм [1902] nicht bestätigt werden.

5. Chydorus sphaericus O. F. MülLer.

Fundorte: Achensee, Attersee, Berglsteinersee, Bodensee, Erlaufsee, Faistenauer Hintersee, Gafiensee, Grundlsee, Hallstättersee, ? Königssee, Kreuthsee, Lunzer Mitter-, Ober- und Untersee, Lünersee, Nassereithersee, Niedertrumersee, Vorderer Oberbergersee, Partnunersee, Pillersee, Unterer Seebisee, Starnbergersee, Tilisunasee, Wallersee, Zireinersee (Nördl. Kalkalp.); Anderlesee, Kleiner See südlich vom Anderlesee und Kleiner See westlich vom Anderlesee, Auerlingsee, Berghaustümpelsee, Hinterer Finstertalersee, Oberer Giglachsee, Grünsee, Oberer Klaftersee, Klammjochsee, Mittlerer Klippensee, Lambrechtersee, Unterer Lanischsee, Lansermoorsee, Millstättersee, Oberer und Unterer Mühldorfersee, Mutterbergersee, Unterer Plenderlesee, Reithersee, Saualmsee, Schlickersee, Schwarzsee bei Turrach, Unterer Torjochsee, Turrachersee, Zellersee, Zirmsee (Zentralalp.); Großer und Kleiner Bödensee, Bc ësee, Faakersee, Gardasee, Kalterersee, Großer Montigglersee, Tovelsee, Oberer Weißenfelsersee, Wocheinersee (Südl. Kalkalp.).

Im Zellersee wurde neben der Type auch die individuelle Variation coelatus Stivg. gefunden.

Auftreten: Vorwiegend in der Küstenzone lebend, meist nahe am Grunde, in Seichtwasser und bis in $80 \mathrm{~m}$ Tiefe beobachtet; als Planktonbestandteil speziell im Hinteren Finstertalersee, Grünsee, Oberen und Unteren Mühldorfersee, Lunzer Mitter- und Lunzer Untersee konstatiert. Höchstgelegene Fundorte: Gafiensee $(2312 \mathrm{~m})$, Zirmsee $(2500 \mathrm{~m})$ und Großer und Kleiner Bödensee (2300 m).

\section{Daphnia.}

Analog dem Genus Bosmina ist auch bei dieser Gattung die Benennung der Spezies nach der von KEILHAck [1909] geschaffenen Grundlage vorgenommen worden; die Namen, welche ältere Autoren ver- 
wendet haben, sind aus den einzelnen Faunenlisten des vorhergehenden Abschnittes zu entnehmen. Alle speziellen Angaben vom Nachweis bestimmter Varietäten und formae wurden angemerkt. Daß unter dem Namen Daphnia longispina nicht immer auch die var. longispina s. str. gemeint gewesen war, braucht nicht erst betont zu werden; daher geben die bisher bekannten Fundorte noch kein vollständig entsprechendes Bild über die Verteilung der Form in den Ostalpen. Für andere Variationen der Daphnia longispina werden weiter fortgesetzte Untersuchungen zweifellos noch wesentlich größere Verbreitungsgebiete feststellen, als sie sich nach den bisherigen Meldungen ergeben. Mit Ausnahme von D. pulex haben alle Daphnia-Formen einen hervorragenden Anteil an der Zusammensetzung des Planktons. Das Vorkommen der Gattung (ohne nähere Bezeichnung der Form) wurde für folgende Seen konstatiert: Altausseersee, Attersee, Bannwaldsee, Brennsee, Faakersee, Grundlsee, Hallstättersee, Keutschachersee, Klopeinersee, Königssee, Königsangersee, Krottensee, Vorderer Langbathsee, ? Unterer Lanischsee, Leonhardsee, Lunzer Mittersee, Millstättersee, Nussensee, Obersee beim Königssee, Ödensee, Offensee, Ossiachersee, Schwansee, Schwarzsee am Schafberg, Seebachsee, Sonthofersee, Spitzingsee, Staffelsee, Tegernsee, Turrachersee, Walchensee, Weißensee bei Füßen, Wocheinersee, Wolfgangsee, Worstnigsee, Wörthersee, Zellersee. Davon sind später mehrfach genauere Angaben über die Spezies nachgetragen worden, doch mußte der ursprüngliche Nachweis des Genus in dieser Liste erwähnt werden.

1. Da phnia longispina 0 . F. Müller.

Bisher genannt für: Altausseersee, Augstsee, Bodensee, Fuschlsee, Garschinasee, Vorderer Gosausee, Leopoldsteinersee, Lunzer Mittersee, Lunzer Obersee, Lünersee, Offensee, Partnunersee, Plansee, Tilisunasee, Traunsee, Wallersee (Nördl. Kalkalp.); Gaishornsee, Haidersee (Zentralalpen); Alleghesee, Iseosee, Lagoraisee, Seraiasee (Südl. Kalkalp.).

Auftreten: Pelagisch an der Oberfläche und bis in $30 \mathrm{~m}$ Tiefe beobachtet. Lebt auch in Seen des Hochgebirges.

2. Daphnia longispina var. cristata G. O. SARS.

Bisher aus zwei Seen der Südlichen Kalkalpenzone gemeldet: Idrosee und Keutschachersee; in dem letzteren speziell als forma cederströmi SCHOEDLER bezeichnet.

3. Daphnialongispina var. cueullata G. O. SARS (=Hyalodaphniacucullata autorum).

Als forma kahlbergensis ScHOEDLER nachgewiesen im: Hopfensee, Krottensee, Mondsee, Wolfgangsee (Nördl. Kalkalp.); Ossiachersee 
(Zentralalp.); Faakersee, Keutschachersee, Klopeinersee, Veldessee, Wörthersee (Südl. Kalkalp.).

$\mathrm{Al}_{\text {s }}$ forma apicata KURz beobachtet im Veldessee (Südl. Kalkalp.).

Als forma sarsi Laxghans genannt für Mondsee und Wolfgangsee (Nördl. Kalkalp.).

Als forma incerta RIchaRd angegeben für den Wörthersee.

Als forma typica (bezw. ohne nähere Bezeichnung!) außerdem genannt für: Simssee (Nördl. Kalkalp.); Millstättersee und Ossiachersee (Zentralalp.); Wörthersee (Südl. Kalkalp.).

Aus der Zusammenstellung ist ersichtlich, daß mehrfach 2, in einem Falle sogar 3 formae zusammen an einem und demselben Fundorte vorkommen.

Bisher im Hochgebirge nicht beobachtet; vielmehr gehören alle aufgezählten Seen den niedrigen Höhenlagen (439-785 m ü.d. M.) an, zeichnen sich jedoch durch größere Tiefen aus, da selbst der Kleine Krottensee $45,5 \mathrm{~m}$ hinabreicht.

4. Daphnia longispina var. hyalina LEYDIG $(=D$. hyalina a u tor $\mathrm{m}$ ).

Fundorte für die forma typica (bezw. ohne Angabe einer bestimmten Form): Achensee, Alpsee bei Immenstadt, Altausseersee, Attersee, Bodensee, Chiemsee, Erlaufsee, Faistenauer Hintersee, Grundlsee, Hallstättersee, Hintersee bei Ramsau, Königssee, Krottensee, Vorderer Langbathsee, Lunzer Untersee, Schliersee, Simssee, Tegernsee, Traunsee, Wolfgangsee (Nördl. Kalkalp.); Piburgersee, Schwarzsee bei Turrach, Zellersee (Zentralalp.); Kalterersee, Keutschachersee, Klopeinersee, Großer und Kleiner Montigglersee, Tovelsee (Südl. Kalkalp.).

Ferner als forma eylmanni Burckhardt im Bodensee nachgewiesen. Als forma foreli Burckнardt im Dürrensee und Pragsersee (Südl. Kalkałp.);

als forma galeata SARS im Ammersee (Nördl. Kalkalp.), Faakersee, Revinesee, Veldessee, Wörthersee (Südl. Kalkalp.);

als forma gracilis HeLLich im Mondsee (Nördl. Kalkalp.) und Ossiachersee (Zentralalp.);

als. forma microcephala G. O. SARS im Misurinasee (Südl. Kalkalp.); als forma pavesii BuRcKHARDT im Gardasee (Südl. Kalkalp.);

als forma pellucida P. E. Müller im Ammersee (Nördl. Kalkalp.) und Millstättersee (Zentralalp.).

Aus dieser Zusammenstellung ist ersichtlich, daß in einigen Fällen 2 formae in einem Wasserbecken gleichzeitig nachgewiesen wurden. 
D. longispina var. hyalina ist bisher in keiner Form aus dem eigentlichen Hochgebirge oberhalb der Waldgrenze für das Ostalpengebiet bekannt.

5. Daphnia longispina var. longispina s. str.

Fundorte: Kleiner See südlich vom Anderlesee, Kleiner See westlich vom Anderlesee, Berghaustümpelsee, Klammjochsee, Saualmsee, Saureggersee, Torjochsee (Zentralalp.); Großer und Kleiner Bödensee, Bo ësee, Wörthersee (Südl. Kalkalp.).

Für den Klammjochsee wird speziell die forma rosea-cavifrons G. O. SARS, für den Wörthersee die forma caudata G. O. SARS genannt.

Diese Varietät der D. longispina scheint demnach in den Hochgebirgsseen des Ostalpengebietes besonders häufig vertreten zu sein.

6. Daphnia pul ex (DE GEER).

Bisher nachgewiesen im Bodensee, Lünersee (Nördl. Kalkalp.); Alleghesee, Boësee, Idrosee, Iseosee, Seraiasee (Südl. Kalkalp.).

Auftreten: Planktonisch und litoral. Steigt ins Hochgebirge (Lünersee $1943 \mathrm{~m}$, Boësee $2282 \mathrm{~m}$ ü. d. M.).

7. Daphnia pulex var. middendorffiana (FISCHER).

Bisher nur im Anderlesee (Zentralalp., $2000 \mathrm{~m}$ ü.d. M.) gefunden. 8. Daphnia pul ex var. obtus a Kurz.

Aus dem Augstsee (Nördl. Kalkalp.), Boësee und Gardasee (Südl. Kalkalp.) bekannt.

Zur Verbreitung der Daphnia-Arten vergl. Fig. 24 u, 25, Taf. VIII.

\section{Diaphanosoma.}

Von dieser Gattung kommt nur eine Spezies in Betracht, von welcher außer der typischen Form noch zwei Variationen im Bereiche der Ostalpenseen beobachtet wurden; eine der letzteren scheint allerdings nicht sicher begründet zu sein.

1. Diaphanosomabrachyurum (LiÉvin) (= Daphnella. brachyura a to ru m).

Nachgewiesen in folgenden Wasserbecken aller drei Ostalpenzonen: Alpsee bei Füßen, Alpsee bei Immenstadt, Ammersee, Attersee, Bannwaldsee, Bodensee, Chiemsee, Fuschlsee, Hopfensee, Krottensee, Krummsee, Vorderer Lahngangsee, Vorderer Langbathsee, Mondsee, Reintalersee, Schliersee, Schwansee, Schwarzsee am Schafberg, Simssee, Sonthofersee, Staffelsee, Starnbergersee, Toplitzsee, Traunsee, Wallersee, Weißensee bei Füßen (Nördl. Kalkalp.); Auerlingsee, Leonhardsee, Millstättersee, 
Neusiedlersee, Ossiachersee, Schwarzsee bei Kitzbühel, Vassachersee, Zellersee (Zentralalp.); Caldonazzosee, Ceisee, Faakersee, Gardasee, Iseosee, Jeserzersee, Kalterersee, Keutschachersee, Klopeinersee, Lasessee, Lavaronesee, Levicosee, Loppiosee, Großer und Kleiner Magdalenensee, Großer und Kleiner Montigglersee, Piazzesee, Revinesee, Santa Crocesee, Santosee, Seebachsee, Terlagosee, Veldessee, Weißensee bei Greifenburg, Wocheinersee, Worstnigsee, Wörthersee (Südl. Kalkalp.).

Auftreten: Meist im Oberflächenplankton, manchmal dominierend, beobachtet. Die Cladocere, welche im Bereiche der Ostalpen sehr weit verbreitet erscheint, fehlt in den Hochgebirgsseen gänzlich, was mit den Angaben aus den Schweizer Alpen vollkommen übereinstimmt (vergl. Stingelin [1908]; höchstgelegene Fundorte: Vorderer Lahngangsee $(1555 \mathrm{~m})$, Auerlingsee $(1500 \mathrm{~m})$, Lavaronesee (1100 $\mathrm{m}$ ü. d. M.).

2. Diaphanosomabrachyurum var. leuchtenbergia$n$ u $m$ FISCHER.

Speziell erwähnt für: Attersee, Bodensee, Wolfgangsee (Nördl. Kalkalp.) und Veldessee (Südl. Kalkalp.).

3. Diaphanosomabrachyurum var.tridentinum LARGAIOLLI.

Ausschließlich von Largaiolli neben der Type im Lavaronesee, Loppiosee und Terlagosee (Südl. Kalkalp.) beobachtet. Die Berechtigung zur Aufstellung der Varietät erscheint uns zweifelhaft; vergl. dazu LARGatoliti [Archiv f. Hydrobiol. 1906].

Zur Verbreitung von D. brachyurum vergl. Fig. 26, Taf. VIII.

\section{Eurycercus.}

Die Gattung ist nur mit einer, im Litoralgebiet der Seen lebenden Spezies vertreten, nämlich:

Eurycercus la mellatus (0. F. MüLLER).

Bisher beobachtet in folgenden Wasserbecken: Achensee, Attersee, Bodensee, Grundlsee, Hallstättersee, Mariasteinersee, Pillersee, Reintalersee, Traunsee (Nördl. Kalkalp.); Gaishornsee, Millstättersee, Zellersee (Zentralalp.); Faakersee, Großer Magdalenensee, Oberer Weißenfelsersee, Wörthersee (Südl. Kalkalp.). Siehe Fig. 23, Taf. VIII.

Im Litorale des Achensees und des Reintalersees fand BREHm auch in den Wintermonaten unter der Eisdecke eiertragende Weibchen dieser Cladocere; sie wurde bis in $64 \mathrm{~m}$ Tiefe beobachtet. Im Gegensatze zum Vorkommen in den Westalpen (Schweiz) wurde E. lamellatus im Ost- 
alpenbereich nur bis in mäßige Gebirgshöhen (Oberer Weißenfelsersee $940 \mathrm{~m}$, Achensee $930 \mathrm{~m}$ ) nachgewiesen.

\section{Graptoleberis.}

Diese zur Familie der Chydoridae zählende Gattung ist nur durch eine Spezies vertreten, nämlich:

Graptoleberis testudinaria (FISCHER).

Bisher bekannt aus: Attersee, Bodensee, Faistenauer Hintersee, Grundlsee, Hallstättersee, Igelsee, Krummsee, Lunzer Untersee, Niedertrumersee, Pillersee, Reintalersee (Nördl. Kalkalp.); Jeserzsee, Großer Montigglersee (Südl. Kalkalp.); aus der Zentralalpenzone derzeit noch nicht nachgewiesen.

Auftreten: Litoral, im Niedertrumersee auch in der Schlammfazies gefunden. Scheint dem Hochgebirge oberhalb der Waldgrenze zu fehlen.

\section{Iyocryptus.}

Von dem Genus wurde bisher nur eine Spezies im Ostalpenbereiche gefunden.

Ilyocryptus sordidus (LiÉvin).

Bekannt aus dem Lunzer Mittersee (Nördl. Kalkalp.), Leonhardsee (Zentralalp.), Gösselsdorfersee (Südl. Kalkalp); die drei Fundorte gehören zu den seichteren Wasserbecken. Siehe Fig. 23, Taf. V1II.

\section{Latona.}

Im Ostalpenbereiche vertreten durch:

L a t o na se tifera (0. F. MüLler).

Bisher nachgewiesen im Litorale des Bodensees (Nördl. Kalkalp.); Jeserzsee und Keutschachersee (Südl. Kalkalp.). Siehe Fig. 23, Taf. VIII.

Vertreten durch:

\section{Lathonura.}

L a tho nura rectirostris (O. F. MüLLER).

Nur im Igelsee (Nördl. Kalkalp., 621m ü. d. M.) und Gaishornsee (Zentralalp., $700 \mathrm{~m}$ ï. d. M.) beobachtet. Siehe Fig. 23, Taf. VIII.

Vertreten durch:

\section{Leptodora.}

Leptodorakindtii (Focke) (=L. hyalina autoru m).

Fundorte: Alpsee bei Füßen, Alpsee bei Immenstadt, Altausseersee, Ammersee, Attersee, Bannwaldsee, Bodensee, Chiemsee, Eibsee, Fuschlsee, Grundlsee, Hallstättersee, Hopfensee, Krottensee, Vorderer Langbath- 
see, Mondsee, Plansee, Schliersee, Schwarzsee am Schafberg, Simssee, Sonthofersee, Spitzingsee, Staffelsee, Tegernsee, Traunsee, Walchensee, Wallersee, Weißensee bei Füßen, Wolfgangsee (Nördl. Kalkalp.); Millstättersee und Ossiachersee (Zentralalp.); Caldonazzosee, Faakersee, Gardasee, Idrosee, Iseosee, Kalterersee, Keutschachersee, Klopeinersee, Lasessee, Lavaronesee, Levicosee, Großer Montigglersee, Revinesee, Santa Crocesee, Santosee, Terlagosee, Veldessee, Worstnigsee, Wörthersee (Südl. Kalkalp.).

Von den genannten 50 Fundorten gehören nur 2 der Zentralalpenzone an und auch diese fallen in unmittelbare Nähe der Grenzlinie gegen die Südliche Kalkalpenzone; zudem sagen Flächenausmaße und Tiefenverhältnisse dieser beiden großen Seen der Vorliebe des Tieres für gewisse Existenzbedingungen außerordentlich zu. Besonders die Liste der in der Nördlichen Kalkalpenzone liegenden Fundstellen setzt sich aus solchen Seen zusammen, die groß und tief genug zugleich sind. Das seichteste Wasserbecken im Ostalpenbereich, welches Leptodora kindtii enthält, stellt der Kalterersee (5-6 m tief) in Südtirol dar; bezüglich des Flächenausmaßes gehen nur einzelne Seen unter das Minimum von 1 km Länge herab (z. B. Krottensee, Lavaronesee, Santosee, Spitzingsee, Worstnigsee), besitzen dafür jedoch Tiefen von 13-45,5 m. Die Cladocere erreicht im Spitzingsee (Nördl. Kalkalp.; $1075 \mathrm{~m}$ ü. d. M.; $15 \mathrm{~m}$ Tiefe) und im Lavaronesee (Südl. Kalkalp.; $1100 \mathrm{~m}$ ü. d. M.; 15,8 m Tiefe) die vertikal am höchsten liegenden Standorte, die bisher aus den Ostalpen bekannt geworden sind. Siehe Fig. 26, Taf. VIII.

Leptodora wurde häufig im Oberflächenplankton und bis in $25 \mathrm{~m}$ Tiefe vorkommend beobachtet, fehlt jedoch in den Wintermonaten (z. B. Hallstättersee und Ossiachersee).

\section{Leydigia.}

Bisher nur in einer Spezies aus dem Ostalpengebiete bekannt, nämlich:

\section{Leydigia a canthocercoides (FISCHER).}

Einziger derzeit erwähnter Fundort: Tilisunasee (Nördl. Kalkalp.; 2102 m ü. d. M.).

\section{Lynceus.}

Das Vorkommen dieses Genus wird für den Chiemsee, Offensee (Nördl. Kalkalp.) und Dürrensee (Südl. Kalkalp.) gemeldet, bezieht sich jedoch keineswegs auf die unter dem Namen Limnetis (brachyura) bekannte Cladocere, sondern auf andere, heute verschiedenen Gattungen 
zugewiesene Formen, die aus der betreffenden Literaturangabe nicht festzustellen sind.

\section{Macrothrix.}

Die Arten dieser Gattung leben vornehmlich am Grunde und im Schlamme der Wasserbecken. Es sind im Bereiche der Ostalpen alle drei von KeIlHack [1909] aufgezählten Spezies gefunden worden, jedoch so selten, daß unsere Kenntnisse von ihrer horizontalen und vertikalen Verbreitung als vollkommen mangelhafte bezeichnet werden müssen.

1. Macrothrixhirsuticornis Norman et Brady.

Fundorte: Bodensee. Lünersee, Partnunersee, Unterer Seebisee, Tilisunasee (Nördl. Kalkalp.); die vier letzten Seen liegen in der Hochgebirgsregion.

2. Macrothrix laticornis (JuRINe).

Fundorte: Bodensee, Tilisunasee (Nördl. Kalkalp.); Neusiedlersee (im Randgebiet der Zentralalpen).

3. Macrothrix rosea (JuRINE).

Fundorte: Vorderer Oberbergersee (Salzkammergut) und Großer Magdalenensee (Klagenfurter Becken).

\section{Moina.}

Aus den Seen des hier behandelten Ostalpengebietes überhaupt nicht bekannt, jedoch in dem zum Vergleiche herangezogenen, am Westrande der Zentralalpen anliegenden $\mathrm{N}$ e u si e d l e r s e e mit der Spezies Moina brachiata (JURINE) vertreten.

\section{Monospilus.}

Grund- und Schlammbewohner, vertreten durch:

Monospilus dispar G. O. SARS (=M. tenuirostris (FISCHER).

Bisher nachgewiesen im: Achensee (Nördl. Kalkalp); Zellersee (Zentralalp.); Großer Magdalenensee, Seebachsee, Wocheinersee (Südl. Kalkalp.). - Noch in $64 \mathrm{~m}$ Tiefe des Achensees gefunden.

\section{Peracantha.}

AusschlieBlich vertreten durch:

Peracantha truncata (0. F. Müller (= Pleuroxus truncatus autorum, auch Peratacantha $t$ r.).

Fundorte: Achensee, Attersee, Berglsteinersee, Chiemsee, Faistenauer Hintersee, Grundlsee, Igelsee, Königssee, Krummsee, Lunzer Obersee, 
Lunzer Untersee, Mariasteinersee, Mittersee am Fernpaß, Niedertrumersee, Reintalersee, Traunsee, Wallersee (Nördl. Kalkalp.); Gaishornsec, Lambrechtersee, Leonhardsee, Millstättersee, Schwarzsee bei Turrach, Vassachersee, Zellersee (Zentralalp.); Ceisee, Gösselstorfersee, Jeserzsee, Großer Magdalenensee, Großer Montigglersee, Santosee, Seebachsee, Tihojasee, Veldessee (Südl. Kalkalp.).

Auftreten: Vorwiegend im Litorale und im Schlammgrund der Wasserbecken, im Reintalersee auch in den Wintermonaten unter der Eisdecke beobachtet. In Chiemsee, Lunzer Obersee und Vassachersee als Planktonbestandteil konstatiert; dies entspricht kaum normalen und konstanten Verhältnissen! Höchstgelegene Fundorte: Schwarzsee bei Turrach $(1850 \mathrm{~m})$, Lunzer Obersee (1113 m), Mittersee am Fern (1086 m) und Lambrechtersee $(1050 \mathrm{~m})$. Bisher somit im Hochgebirge oberhalb der Waldgrenze nicht gefunden.

\section{Pleuroxus.}

Das Vorkommen des Genus (ohne Bestimmung der Spezies) wurde für zwei Seen des Ostalpengebietes nachgewiesen, nämlich für den Kalterersee (Südl. Kalkalp.) und Traunsee (Nördl. Kalkalp.). Die Arten leben vorwiegend litoral und am Grunde der Wasserbecken, auch im Schlamm; es wurden bisher 4 Spezies angetroffen, doch ist die Zahl der Fundorte gering.

1. Ple uroxus a duncus (Jurine).

Fundorte: Bodensee, Königssee, Kreuthsee (Nördl. Kalkalp.).

2. Pleuroxus laevis G. O. SARS.

Bisher nur aus dem Grundlsee (Nördl. Kalkalp.) bekannt.

3. Pleuroxus trigonellus (O. F. MülLer).

Fundorte: Attersee, Lunzer Obersee, Reintalersee (Nördl. Kalkalp.); Loppiosee, Großer Magdalenensee (Südl. Kalkalp.). - Das Auftreten (ler Spezies im Lunzer Obersee (1113 m ü. d. MI.) wird als planktonisch bezeichnet!

4. Pleuroxus uneinatus BAIRD.

Fundorte: Ammersee, Bodensee, Grundlsee, Wallersec (Nördl. Kalkalp.); Lambrechtersee, Zellersee (Zentralalp.). - Aus dem Wallersee in $10 \mathrm{~m}$ Tiefe, aus dem Zellersee in der Schlammfazies konstaticrt.

\section{Polyphemus.}

Diese auffällige Cladocere aus der Familie der Polyphemiden wurde in den Seen der Ostalpen bereits mehrfach nachgewiesen; die Funde beziehen sich auf die bekannte Spezies

Archiv f. Hydrobiologie. Suppl.-Bd. III. 
Polyphemus pediculus (Linné).

Bisher beobachtet im: Bodensee, Erlaufsee, Kammersee, Vorderer Lahngangsee, Vorderer Langbathsee, Lunzer Obersee, Ödensee, Traunsee (Nördl. Kalkalp.); Großer Bödensee, Kleiner Bödensee, Gardasee, Raiblersee (Südl. Kalkalp.). Aus der Zentralalpenzone noch nicht gemeldet.

Die von $P$. pediculus bewohnten Fundorte beweisen, daß sich diese Cladocere weder in bezug auf die Größe, noch in bezug auf die Tiefe. des Wasserbeckens wählerisch zeigt; nach LAUTER Bons [1913] soll die Form am regelmäßigsten in Moortümpeln zu finden sein! In Abhandlungen über tiergeographisch-faunistische Fragen wird $P$. pediculus öfter genannt; EkMaN [1904] zählte ihn zu den nordöstlichen Elementen einer boreosubglazialen Faunengruppe, hielt diese Zugehörigkeit jedoch für nicht sicher; Brenm [1907, Zool. Anz., vol. 31, p. 320] hingegen glaubt durch seine Untersuchungen in den Seen der Ostalpen den Beweis für die Richtigkeit der Anschauung Ekman's erbracht zu haben. ZachARIAS [1906, Zool. Anz., vol. 30, p. 455] äußerte sich dahin, daß man diese Cladocere zweifellos noch vielfach in der Schweiz und in Tirol antreffen werde; dieser Meinung setzte Brenm [1907 op. cit.] die „Überzeugung" entgegen, daß „Vorkommnisse der genannten Cladocere im Alpengebiet auf vereinzelte seltene Fälle von Verschleppung zurückzuführen sind".

Die von uns hier mit Absicht ausführlicher angegebenen Zitate mögen als Belege dafür dienen, daß derartige Auseinandersetzungen nicht mehr als rein spekulativen Wert beanspruchen können; jede Meldung neuer Fundorte, von denen wir ganz ohne Zweifel immer noch viel zu wenige kennen, vermag mit einem Schlage dem Hypothesenbau solch schwacher Art den Boden zu entziehen. Vergl. dazu Fig. 27, Taf. VIII.

Auftreten: P. pediculus lebt in den genannten Seen teils planktonisch, teils litoral. In vertikaler Verbreitungsrichtung reicht sein Vorkommen im Gebiete vom $64 \mathrm{~m}$ ü. d. M. gelegenen Gardasee bis zu den $2300 \mathrm{~m}$ ü. d. M. gelegenen Bödenseen. Nach Stingelin [1908] aus höher gelegenen A!penseen der Schweiz noch nicht bekannt.

Vertreten durch:

\section{Pseudalona (= Kurzia).}

Pseudalona latissima. (Kunz).

Bisher ausschließlich im Litorale des Großen Montigglersees (Südl. Kalkalp.) nachgewiesen. 


\section{Rhynehotalona.}

Das Genus ist auch im Gebiete der Ostalpen mit den zwei folgenden, zu den litoral- und grundbewohnenden Faunenelementen gehörigen Spezies vertreten, jedoch noch sehr selten gemeldet.

Rhynchotalona falcata (G. O. SARs).

Bisher nur im Litorale des Achensee (Tördl. Kalkalp.) und Terlagosee (Südl. Kalkalp.) nachgewiesen.

Rhynchotalona rostrata (KocH).

Fundorte: Bodensee, Garschinasee, Lünersee, Partnunersee, Tilisunasee, Traunsee (Nördl. Kalkalp.); davon liegen vier Seen in der Hochgebirgsregion oberhalb der Waldgrenze. Das Vorkommen stimmt mit den Angaben STINGELiv's [1908] gut überein, auch bezüglich der Fundorte von $R h$. falcata. Im Lünersee wurde $R h$. rostrata bis in $100 \mathrm{~m}$ Tiefe (Seegrund an der tiefsten Stelle $102 \mathrm{~m}$ ) konstatiert. Vergl. Fig. 27, Taf. VIII.

\section{Scapholeberis.}

Während Scapholeberis cornuta SchoedLer von den meisten Autoren als individuelle Variation der Spezies mucronata (O. F. MülLER) fronte laevi aufgefaßt wird, bleiben die Formen Sc. mucronata var. longicornis Lutz und Sc. obtusa Schoedler (= aurita Fischer!) aufrecht erhalten; in der folgenden Aufzählung ist diesem Standpunkte Rechnung getragen.

Auftreten: Im Litorale der größeren Seen, seltener pelagisch in kleineren Wasserbecken.

Se a pholeberis mucronata (O. F. MüLler).

Fundorte: Achensee, Berglsteinersee, Bodensee, Erlaufsee, Grundlsee, Igelsee, Krummsee, Lunzer Obersee, Schwarzsee am Schafberg, Simssee, Spitzingsee, Wildsee bei Seefeld (Nördl. Kalkalp.); Gaishornsee (Nev-siedlersee), Reithersee, Zellersee (Zentralalp.); Dürrensee, Faakersee, Gardasee, Kleiner Magdalenensee, Veldessee, Wocheinersee (Südl. Kalkalpen). - Höchstgelegener Fundort: Dürrensee (1410 m ü. d. M.).

Scapholeberis mucronata var. longicornis Lutz.

Bisher nur für den Grundlsee (Salzkammergut) gemeldet.

$S c a p h o l e b e r i s$ a urita (Fischer) $(=S c$. obtus a SCHOEdLer e t a u tor u m).

Fundorte: Bisher nur in zwei Seen der Südlichen Kalkalpenzone nachgewiesen, nämlich im Gösselsdorfersee und im Klopeinersee.

Zur Verbreitung von, Scapholeberis vergl. Fig. 25, Taf. VIII. 


\section{Vertreten durch:}

\section{Sida.}

Sida crystallina (O. F. Müller).

Bisher gemeldet aus folgenden 36 Sren des Ostalpenbereiches: Ichensee, Attersee, Bodensee, Faistenauer Hintersee, Grundlsee, Hallstättersee, Igelsee, Krummsee, Mondsee, Niedertrumersee, Reintalersee, Schwarzsee am Schafberg, Spitzingsee, Traunsee, Wallersee, Wolfgangsee (Nördl. Kalkalp.); Lambrechtersee, Lansersee, Millstättersee (Neusiedlersee), Ossiachersee, Vassachersee, Zellersee (Zentralalp.); Ceisee, Faakersee, Gardasee, Gösselsdorfersee, Jeserzsee, Keutschachersee, Klopeinersee, Loppiosee, Großer Magdalenensee, Santa Crocesee, Santosee, Seraiasee, Terlagosee, Worstnigsee (Südl. Kalkalp.). Siehe Fig. 27, Taf. VIII.

Höchstgelegene Fundorte: Spitzingsee (1075 m ü. d. M.); Lambrechtersee (1050 m ü. d. M.); Seraiasee (974 m ü. d. M.). Das Bild von der vertikalen Verbreitung dieser Cladocere im Ostalpengebict unterscheidet sich somit bisher wesentlich von dem in den Schweizer Alpen, wo Sida crystallina in mehreren, oberhalb der Waldgrenze gelegenen Hochgebirgsseen gefunden wurde [vergl. Stingelin 1908]. Von den 36 genannten Seen besitzt der Krummsee die geringste Tiefe $(2,5 \mathrm{~m})$. Auftreten: Vorwiegend als Litoralbewohner konstatiert, im Lansersee und Wallersee jedoch auch als Planktonform gemeldet.

\section{Simocephalus.}

Die Angehörigen dieses Genus leben im allgemeinen an der Küste der Seen und sind nur in seltenen Ausnahmen auch im Plankton beobachtet worden. Von den drei im Gebiete nachgewiesenen Arten wurde bisher nur eine (Simocephalus vetulus) in einer nennenswerten Anzahl von Wasserbecken gefunden.

1. Simocephalus exspinosus (KocH).

Bisher nur im Großen Magdalenensee (Südl. Kalkalp.; Ḱlagenfurter Becken) konstatiert.

2. Simocephalus serrulatus (Косн).

Bisher nur aus dem Sommersbergersee (Nördl. Kalkalp.; Salzkammergut) bekannt.

3. Simocephalus vetulus (O. F. MÜLLer).

Fundorte: Achensee, Bodensee, Faistenauer Hintersee, Grundlsee, Hallstättersee, Igelsee, Königssee, Lunzer Obersee, Lunzer Untersee, Odensee (Nördl. Kalkalp.); Berghaustümpelsee, Gaishornsee, Lambrechtersee, Lansermoorsee, Millstättersee (Zentralalp.); Alleghesee, 
Großer und Kleincr Bödensee. Faakersee, Jeserzsee, Oberer Weißenfelsersee, Wörthersee (Südl. Kalkalp.).

Auftreten: Als Planktorform selten (z. B. Alleghesee, Lunzer Obersee), im Litorale vorwicgend arzutreffen; bewohnt Seen mit großem Areal und großen Tiefen eberso wie solche von sehr geringem Ausmabe und niedrigem Wasserstand, die letzteren allerdings nur bei reichlich entwickelter Wasseıflora; in diesen Fällen steigt $S$. vetulus auch in die Hochgebirgsregion empor (z. B. Bödenseen, $2300 \mathrm{~m}$ ï. d. M.).

\section{Zusammenfassung.}

Nachdem die rertikale Verbreitung der seenbewohnenden Copepoden und Cladoceren bezw. speziell ihr Vorkommen in den über der Waldgrenze gelegenen Hochgebirgswasserbecken der Ostalpen schon am Schlusse des I. Abschnittes dieser „Studien“ zusammenfassend bcsprochen wurde, so bleibt hier noch die Frage zu erörtern, ob es möglich ist, dic horizontale Verbreitung der Spezies mit der geologischpetrographischen Zoneneirteilung des Ostalpenbereiches in Beziehung: zu bringen. Dazu wird es nötig sein, zunächst über die Anzahl und Lage. der Fundorte jeder Spezies eine Utbersicht zu gewinnen; sie kann aus der folgenden Aufzählung, welche zugleich eine Liste der im $b$ handelten Gebicte bisher bekannt gewordenen Formen darstellt, eı.trommen werden.

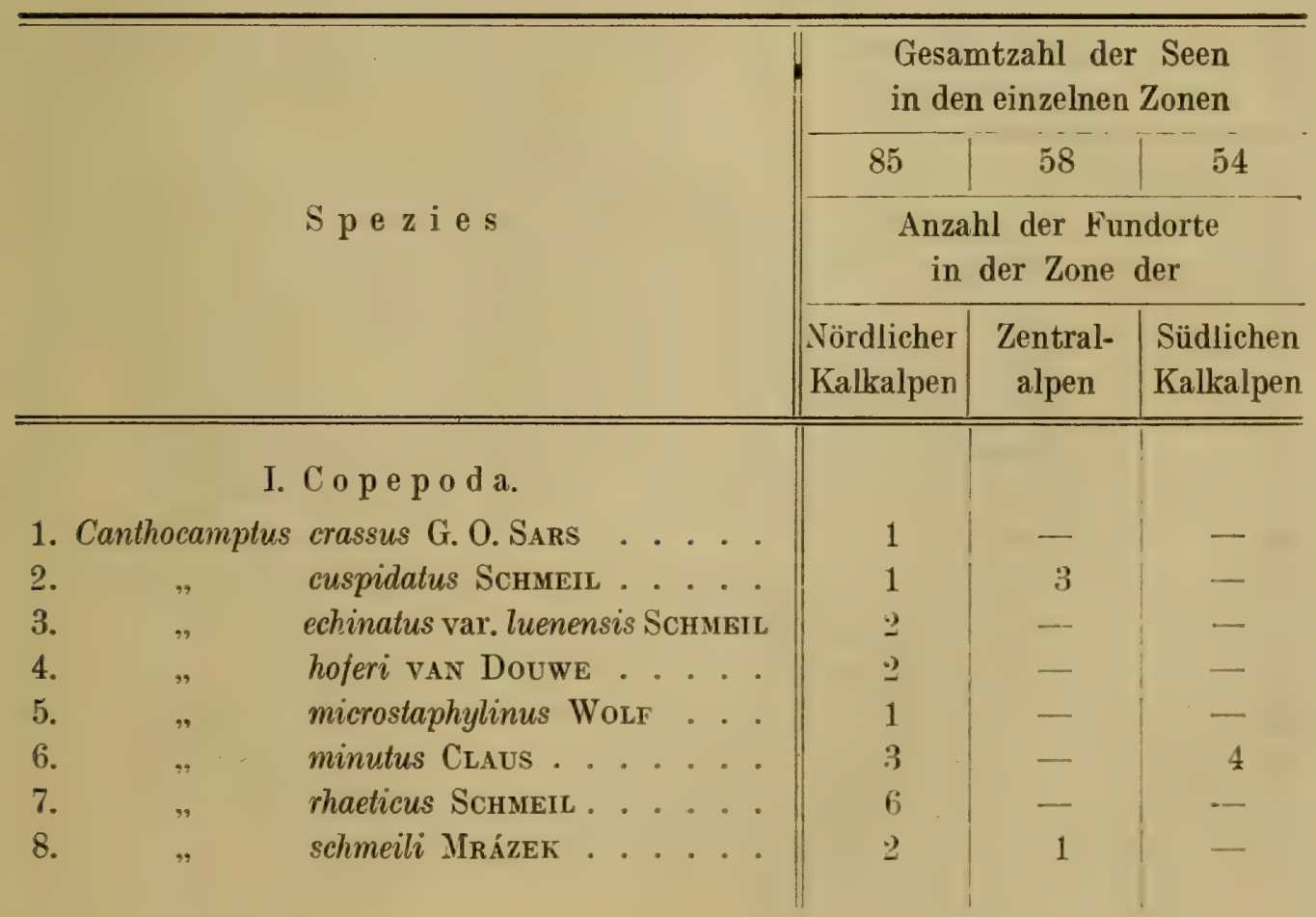




\begin{tabular}{|c|c|c|c|}
\hline \multirow{4}{*}{ Spezies } & \multicolumn{3}{|c|}{$\begin{array}{l}\text { Gesamtzahl der Seen } \\
\text { in den einzelnen Zonen }\end{array}$} \\
\hline & 85 & 58 & 54 \\
\hline & \multicolumn{3}{|c|}{$\begin{array}{l}\text { Anzahl der Fundorte } \\
\text { in der Zone der }\end{array}$} \\
\hline & Nördlichen & $\begin{array}{l}\text { Zentral- } \\
\text { alpen }\end{array}$ & $\begin{array}{l}\text { Südlichen } \\
\text { Kalkalpen }\end{array}$ \\
\hline $\begin{array}{l}\text { 9. Canthocamptus schmeili var. biserialis Mico- } \\
\text { LETZKY . . . . . }\end{array}$ & 1 & 1 & - \\
\hline schmeili var. hamata SchmeIL & 1 & - & - \\
\hline staphylinus (JURINE) . . . . & 6 & - & 2 \\
\hline trispinosus BRADY..... & 1 & - & - \\
\hline vejdowskyi MrázeK . . . . . & 1 & - & - \\
\hline wierzejskii MrázeK . . . . & 5 & - & - \\
\hline zschokkei SchmeIL . . . . & 4 & - & - \\
\hline 16. Cyclops affinis G. 0. SARS. . . . . . & 1 & - & - \\
\hline $17, \quad, \quad$ albidus JURINE $\ldots \ldots \ldots$ & 6 & 3 & 3 \\
\hline bicolor G. O. SARS . . . . . & 1 & - & - \\
\hline diaphanus Fischer . . . . . . . & - & - & 5 \\
\hline fimbriatus Fischer $\ldots . . .$. & 4 & 1 & - \\
\hline fuscus JURINE . . . . . . . & 9 & 2 & 7 \\
\hline insignis Claus . . . . . . & - & - & $1(?)$ \\
\hline leuckarti Cuaus . . . . . . & 4 & 5 & 8 \\
\hline macrurus G. O. SARS . . . . : & 6 & 2 & 3 \\
\hline magniceps LILLJEBORG. ....... & $1(?)$ & - & - \\
\hline oithonoides G. O. SARS . . . . & 1 & - & - \\
\hline serrulatus FISCHER . . . . . & 15 & 18 & 17 \\
\hline serrulatus var. denficulata GRAETER . & 1 & - & - \\
\hline strenuus Fischer . . . . . . . & 22 & $11+(? 1)$ & 17 \\
\hline vernalis FISCHER . . . . . . . & 7 & $10+(? 1)$ & - \\
\hline viridis Jurine . . . . . . . . & 6 & $3(+1)$ & 6 \\
\hline 32. Diaptomus bacillifer Koelbel . . . . . & 6 & 3 & 1 \\
\hline 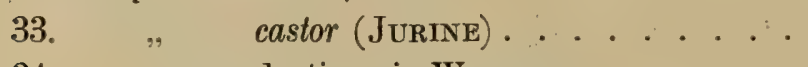 & - & 2 & 3 \\
\hline denticornis WIERZEJSKI ..... & 2 & 2 & 1 \\
\hline gracilis G. 0 . SARS . . . . & $\mid 18(+? 1)$ & 4 & 6 \\
\hline gracilis var. carnicus SENNA. . . & - & - & 1 \\
\hline graciloides LILLJEBoRG . . . . & 2 & - & 1 \\
\hline laciniatus LILLJEBorg . . . . & 3 & - & - \\
\hline laticeps G. 0. SARS . . . . & - & - & 1 \\
\hline spinosus DADAY ....... & - & (1) & - \\
\hline steueri Brehm et Zederbauer . & - & - & 2 \\
\hline tatricus WieRzeJSKI $\because \ldots$. & - & 1 & 一 \\
\hline vulgaris SchMeIL ....... & - & - & 1 \\
\hline 44. Heterocope saliens LillúbBorg . . . . . & 4 & 5 & - \\
\hline 45. " veismanni Імног . . . . . . & 4 & - & - \\
\hline 46. Moraria schmeili van Douwe...... & 3 & - & - \\
\hline
\end{tabular}




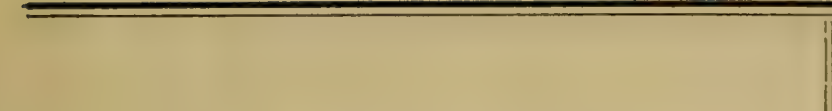

Gesamtzahl der Seen

in den einzelnen Zonen

Spezies

\begin{tabular}{c|c|c}
85 & 58 & 54 \\
\hline & $\begin{array}{c}\text { Anzahl der Fundorte } \\
\text { in der Zone der }\end{array}$
\end{tabular}

Nördlichen Zentral- Südlichen

\begin{tabular}{lll} 
Kalkalpen & alpen & Kalkalpen \\
\hline
\end{tabular}

II. $\mathrm{C}$ I a d o c e r a.

1. Acroperus angustatus G. O. SARS.....

2. ". harpae BAIRD.........

3. " harpae var. frigida EкMAN ....

4. Alona affinis (LEYDIG) . . . . . . .

5. " costata G. O. SARS ........

6. " guttata G. O. SARS ........

7. " quadrangularis (O. F. MÜLLER) ...

8. " rectangula G. O. SARS........

9. " tenuicaudis G. O. SARS .......

10. Alonella excisa (FISCHER) . . . . . . . .

11. " exigua (LILLJEBORG). . . . . . .

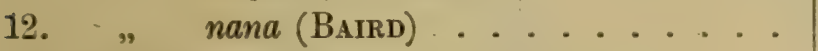

13. Alonopsis elongata G. O. SARS . . . . .

14. Bosmina coregoni BAIRd [BURcKHARdT]...

15. " longirostris (O. F. Müller) [BURCKH.]

16. Bythotrephes longimanus LEYDIG ......

17. Camptocercus lilljeborgi SCHOEDLER . . . .

18. " rectirostris (SchoEdLER)....

19. $" \quad$ rectirostris var. biserratus

(SCHOEDLER) . . . . .

20. Ceriodaphnia laticaudata P. E. MüLLER . .

21. " megops G. O. SARS .....

22. " $" \quad$ pulchella G. O. SARS ....

23. " quadrangula (O. F. MülleR) .

24. " quadrangula var. hamata LILLJ.

25. $" \quad$ reticulata (JURINE) .....

26. Chydorus gibbus LiLlJEborg . . . . . . .

27. " globosus BATRD. .........

28. " latus G. O. SARS .......

29. " piger G. O. SARS ........

30. " sphaericus 0. F. MüLler.....

31. Daphnia longispina O. F. MÜLLER ....

32. " longispina var. cristata G. O. SARS .

33. " " " cucullata G. O. SARS

34. " $\quad " \quad "$ " hyalina LEYDIG..

35. " " " longispina s. str. . .

36. " pulex (DE GEer)........

\begin{tabular}{l|l} 
Ka & alkatpen \\
\hline
\end{tabular}

37. " " var. middendorffiana (FISCHER)

\begin{tabular}{l|l|l}
6 & 1 & 5
\end{tabular}

5

\begin{tabular}{l|l|l}
26 & 14 & 6
\end{tabular}

\begin{tabular}{r|r|r}
1 & - & -
\end{tabular}

\begin{tabular}{l|l|l}
12 & 2 & 4
\end{tabular}

$11-3$

\begin{tabular}{l|l|l}
12 & 5 & 8
\end{tabular}

\begin{tabular}{l|l|l}
7 & 2 & 4
\end{tabular}

- $\quad-\quad 1$

\begin{tabular}{l|l|l}
4. & 4 & 3
\end{tabular}

2 1 1 -

\begin{tabular}{l|l|l}
7 & 2 & 2
\end{tabular}

\begin{tabular}{r|r|r}
8 & - & - \\
12 & 1 & 3
\end{tabular}

\begin{tabular}{l|l|l}
11 & 5 & 21
\end{tabular}

\begin{tabular}{l|l|l}
16 & 2 & 2
\end{tabular}

$\begin{array}{lll}2 & -\end{array}$

-

\begin{tabular}{c|r|r}
1 & - & - \\
2 & - & - \\
- & 1 & - \\
8 & 3 & 8 \\
2 & 1 & 7 \\
4 & - & - \\
1 & - & 1 \\
3 & - & - \\
3 & - & - \\
1 & - & 2 \\
$(? 1)$ & - & 1 \\
$24+(? 1)$ & 27 & 10 \\
16 & 2 & 4 \\
- & - & 2 \\
5 & 2 & 5 \\
22 & 5 & 14 \\
- & 7 & 4 \\
2 & - & 5 \\
- & 1 & -
\end{tabular} 


\begin{tabular}{|c|c|c|c|}
\hline \multirow{4}{*}{ Spezies } & \multicolumn{3}{|c|}{$\begin{array}{l}\text { Gesamtzahl der Seen } \\
\text { in den einze'nen Zonen }\end{array}$} \\
\hline & 85 & 58 & 54 \\
\hline & \multicolumn{3}{|c|}{$\begin{array}{l}\text { Anzahl der Fundorte } \\
\text { in der Zone der }\end{array}$} \\
\hline & $\begin{array}{l}\text { Nördlichen } \\
\text { Kalkalpen }\end{array}$ & $\begin{array}{c}\text { Zentral- } \\
\text { alpen }\end{array}$ & $\begin{array}{l}\text { Südlichen } \\
\text { Kalkalpen }\end{array}$ \\
\hline 38. Daphnia pulex var. obtusa Kurz. & 1 & - & 2 \\
\hline 39. Diaphanosoma brachyurum (Liévin) & 26 & $7+(1)$ & 28 \\
\hline 40. " br. var. leuchtenbergianum Fischer. & 3 & - & 1 \\
\hline$" \quad$ " tridentinum Largajoldi . . & - & - & 3 \\
\hline 42. Eurycercus lamellatus (O. F. MÜLlek). . . . & 9 & 3 & 4 \\
\hline 43. Graptoleberis testudinaria (FISCHER) . . . & 11 & - & 2 \\
\hline 44. Ilyocryptus sordidus (LIÉviN) . . . . . . & 1 & 1 & 1 \\
\hline 45. Latona setifera (O. F. MÜLLER) . . & 1 & $\cdots$ & 2 \\
\hline 46. Lathonura rectirostris (O. F. MüLLER) . . & 1 & 1 & - \\
\hline 47. Leptodora kindtii (FocKE) . . . . & 29 & ? & 19 \\
\hline 48. Leydigia acanthocercoides (FISCHER) . . . . & 1 & 一 & - \\
\hline 49. Macrothrix hirsuticornis NoRMAN et BRADY . & 5 & - & - \\
\hline 50. $\quad " \quad$ laticornis (JURINE) . . . . . & 2 & (1) & - \\
\hline rosea (JURINE) . . . . . . & 1 & - & 1 \\
\hline 52. Moina brachiata (JuRINE) & - & (1) & - \\
\hline 53. Monospilus dispar G. O. SARS . . & 1 & 1 & 3 \\
\hline 54. Peracantha truncata (O. F. MüLLER) . . . & 17 & 7 & 9 \\
\hline 55. Pleuroxus aduncus (JuRINE) . . . . . . & 3 & - - & - \\
\hline 56. $\quad, \quad$ laevis G. 0. SARS ........ & 1 & - & - \\
\hline trigonellus (0. F. MÜLLER) . . . & 3 & $\rightarrow$ & 2 \\
\hline uncinatus BAIRD ....... & 4 & $\ddot{2}$ & - \\
\hline 59. Polyphemus pediculus (Linvé) . . . . . . & 8 & - & 4 \\
\hline 60. Pseudalona latissima (KURz) . . . . . . & - & - & 1 \\
\hline 61. Rhynchotalona falcata (G. O. SARS) . . . & 1 & - & 1 \\
\hline rostrata $($ Косн) .... & 6 & - & - \\
\hline 63. Scapholeberis mucronata (0. F. MüLLER). . & 13 & $2+(1)$ & 6 \\
\hline " var. longicornis LuTz & 1 & - & - \\
\hline aurita (FISCHER) . . . . & 一 & - & 2 \\
\hline 66. Sida crystallina (O. F. Müller) . . . . & 16 & $6+(1)$ & 14 \\
\hline 67. Simocephalus exspinosus (Косн) . . . . & - & - & 1 \\
\hline 68. . " serrulatus (Косн) . . . . & 1 & - & - \\
\hline 69. $\quad " \quad$ vetulus (O. F. MÜLleR) . . . & 10 & 5 & 7 \\
\hline
\end{tabular}

Aus einer Betrachtung über die horizontale Verbreitung der Spezies in dem vorhin angedeuteten Sinne werden jene Formen ganz auszuschalten sein, von welchen angenommen werden kann, daß uns ihr Vorkommen noch vielfach entgangen ist (z. B. Harpacticiden, viele Cladoceren) und daher die Anzahl der Fundorte aus diesem Grunde unzureichend ist. 
In anderen Fällen besitzen vereinzelte Nachweise einer Spezies zweifellos darin ihren Grund, daß die betreffende Form den Seen des Alpengebietes überhaupt im allgemeinen fremd ist (z. B. Diaptomus tatricus, spinosus; Pseudalona latissima). Nicht zuletzt wird auBerdem zu ber ücksichtigen sein, daß auf die Zone der Nördlichen Kalkalpen die absolut größte Fundortsanzahl (85) entfällt, während diese für die Zentralalpen und die Zone der Südlichen Kalkalpen annähernd gleich ist (58 und 54); hierin liegt eine gewisse Ungleichwertigkeit der Nachweise. Zur Aufstellung eines brauchbaren Vergleiches sollen deshalb nur solche Formen herausgehoben werden, von welchen eine größere Anzahl von Fundorten bekannt ist und ein Übersehen beim Aufsammeln des Materiales kaum angenommen werden kann. Demnach zeigt das Bild der horizontalen Verbreitung folgender Spezies ein Dominieren in einzelnen Zonen:

1. Die Zone der Nördlichen Kalkalpen bevorzugt:

Diaptomus gracilis,

Heterocope weismanni.

Alonopsis elongata,

Bosmina coregoni,

Bythotrephes longimanus;

diesen Formen reihen sich verniutlich Macrothrix hirsute cornis und Rhynchotalona rostrata an.

2. Die Zone der Südlichen Kalkalpen bevorzugt:

Cyclops diaphanus.

3. Die Zonen der Nördlichen und Südlichen Kalkalpen bevorzugt: Alona guttata,

Bosnina lonsirostris,

Ceriodaphnia pulchella,

Daphnia longispina var. hyalina,

Diaphanosoma brachyurum,

Leptodora kindtii,

Polyphemus pediculus,

Scapholeberis mucronata,

Sida crystallina;

für Graptoleberis testudinaria ciüfte dasselbe zutreffen.

4. Ein dominierendes Auftreten im Zentralalpengebiet kann vorr keiner Spezies konstatiert werden; für die in der Literatur als charakteristisches Faunenelement der "Urgebirgs"seen bezeichnete Cladocere Holopedium gibberum fehlt bisher überhaupt ein Nachweis im Bereiche der Ostalpen! 
In der Zusammenstellung der obigen Verbreitungsdaten einiger Spezies-kommen somit tatsächliche Unterschiede in der horizontalen Verteilung nach den petrographischen Hauptzonen $\mathrm{zum} \mathrm{Ausdruck}$, die zukünftige Untersuchungen noch ergänzen und erweitern werden. Eine kritische Utberlegung wird nun die Frage zu stellen haben, ob diese Verteilung auf die Unterschiede im Chemismus der Gewässer, der hier kurzerhand aus dem Bau der Hauptgesteinsarten erschlossen erscheint, zurückzuführen ist; mit Rücksicht auf die Ergebnisse, welche unsere Wasseranalysen aus einigen Hochgebirgsseen geliefert haben (vergl. den II. Abschnitt dieser „Studien“), hat diese Frage zunächst dahin beantwortet zu werden, daß der chemische Charakter eines Seewassers nicht in allen Fällen mit Sicherheit aus der geologischpetrographischen Lage des Fundortes hervorgeht, wi e di e s b i s h e r dureh die allgemein übliche Unterseheidung zwischen „Urgesteins"-und, „Kalkgesteins"seen zum A u sdruckgebracht wurde; zudem sind wir über chemische Untersuchungsresultate für Seen in niedereren Höhengürteln der Ostalpen noch sehr wenig unterrichtet. Abgesehen von diesem Einwand, wird die vorhin féstgestellte zonare Verteilung gewiß in vielen Fällen durch andere Ursachen begründet sein; so bilden z. B. die größeren, Seen des Ostalpenrandes bezüglich ihrer bathymetrischen Verhältnisse eine ziemlich einheitliche Gruppe; an diese gemeinsame Milieueigenschaft dürfte das Auftreten von Bythotrephes gebunden sein. Die Verbreitung mancher Arten werden andererseits gemeinsame thermische Faktoren bestimmen, welche gleichzeitig mit einer Zone korrespondieren (Südl. Kalkalp.; Auftreten von Cyclops diaphanus). Es best eht so mit kein stichhaltiger Grund, für das auf bestimmte Ostalpenzonen beschränkte oder dominierende Vorkommen einzelner Copepoden und Cladoeeren ausschlieBlich den ge ologisch-petrographischen Bau des. betreffenden Gebietes verantwortlich zu machen, solange nicht dureh die chemische Analyse eine solehe Abhängigkeit einwandfrei be wiesen wird. Die erkannte zonare. Verbreitung erlaubthingegen sehr wohl, von „Leitformen“bestimmter Zonen im topographischen Sinne zu sprechen. 


\section{Abschnitt.}

\section{Literaturnachweise.}

Baumbach, Рн. 1922: „Studien über das Zooplankton des Chiemsees". Arehiץ für Naturgesch. 88. Jahrg. Abtlg. A. 7. Heft, p. 177 [Abschn. 4].

BlaAs, J. 1902: "Geologischer Führer durch die Tiroler und Vorarlberger Alpen". Innsbruck, Verlag WAGiNer [Abschn. 1 u. 4].

Bӧнм, A. 1886: „Die Hochseen der Ostalpen“. Mitteilg. Greograph. Gesellsch. Wien, Jahrg. 1886, p. 1-23 [Abschn. 3 u. 4].

- 1887: „Einteilung der Ostalpen“. Geograph. Abhandlg., herausg. v. Prof. Dr. A. Penck in Wien, vol. 1, Heft 3, p. 243 [Abschn. 3 u. 4].

Ввенм, V. 1902: „Zusammensetzung, Verteilung und Periodizitit des Zooplanktons im Achensee“. Zeitschr. Ferdinandeum Innsbruck, 3. Folge, vol. 46, p. 33. (Enthält auch Angaben über den Reintalersee) [Abschn. 4 u. 5].

- 1906: „Zur Planktonfauna des Gardasees“. Archiv f. Hydrobiol., vol. 1, p. 496 [Abschn. 4].

- 1906: „Untersuchungen über das Plankton einiger Seen der nördlichen und östlichen Alpen“; „Richtigstellung“". Verhandlg. zool.-botan. Gesellsch. Wien, vol. 66, p. 33 u. p. 340 . (Enthält Angaben über den Chiemsee, Hintersee bei Ramsau, Königssee, Raiblersee, Schwarzensee i. Zillertal.) [Abschn. 4].

- 1907: „Die biologische Süßwasserstation zu Lunz-Seehof, Nied.-Österr.“. Archiv f. Hydrobiol., vol. 2, p. 465 [Abschn. 4].

- 1907: "Beiträge zur faunistischen Durchforschung der Seen Nordtirols". Berichte d. naturw.-medizin. Vereins Innsbruck. vol. 31 (1907/08, p. 97 [Abschn. 1 u. 4].

- 1907: „Über das Yorkommen von Diaptomus tatricus Wierz. in den Ostalpen ...". Zool. Anz., vol. 31, p. 319. (Enthält Angaben über das Vorkommen von Polyphemus pediculus) [Abschn. 4 u. 5].

- 1909: "Charakteristik der Fauna des Lunzer Mittersees“. Internat. Rev. f. Hydrobiol., vol. 2, p. 741 [Abschn. 4].

- 1909/10: „Das Plankton der Alpenseen“. Die Kleinwelt, 1. Jahrg. Heft 5; Bamberg. (Nicht zugänglich!) [Abschn. 4].

- 1912: „Notizen über die Fauna des Achensees in Tirol“. Archiv f. Hydrobiol., vol. 7, p. 687 [Abschn. 4].

- 1913: "Über die Harpacticiden Mitteleuropas“. Archiv f. Hydrobiol., vol. 8, p. 313 u. 575. (Enthält Angaben über den Achensee, Attersee, Eibsee, Erlaufsee, Lunzer Obersee, Lunzer Untersee) [Abschn. 4].

- 1913/14: „Die Fauna der Lunzer Seen, verglichen mit der der anderen Alpenseen“. Internat. Rev. f. Hydrobiol., vol. 6 p. 528 [Abschn. 4].

- 1915: „Zur geographischen Stellung des Diaptomus tatricus". Archiv f. Hydrobiol., vol. 10, p. 405. (Enthält Berichtigung der Determination von Diaptomus laciniatus der Saualpe in D. tatricus) [Abschn. 4 u. 5].

Brehy, V. und Zederbauer, E. 1902: „Untersuchungen über das Plankton des Erlaufsees". Verhandlg. zool.-botan. Gesellsch. Wien, Jahrg. 1902, p. 388 [Abschn. 4].

- - 1904: „Beiträge zur Planktonuntersuchung alpiner Seen“. I. Teil. Verhandlg. zool.-botan. Gesellsch. Wien, vol. 54, p. 48 [Abschn. 4]. 
Breum, V. und Zederbal'er, E. 19(14: „Beiträge zur Planktonuntersuchung alpiner Seen“. II. Teil. Verhandlg. zool.-botan. Gesellsch. Wien, vol. 54, p. 635 [Abschn. 1, 4 u. 5].

- - 1905: „Beiträge zur Planktonuntersuchung alpiner Seen“. III. Teil. Verhandlg. zool.-botan. Gesellsch. Wien, vol. 55, p. 222 [Abschn. 4].

- - 1906: „Beiträge zur Planktonuntersuchung alpiner Seen“. IV. Teil. Verhandlg. zool.-botan. Gesellsch. Wien, vol. 56, p. 19 [Absehn. 4].

- - 1906: „Beobachtungen über das Plankton in den Seen der Ostalpen“. Archiv f. Hydrobiol., vol. 1, p. 469 [Abschn. 4].

Brürsted, J. und WesenberG-Lund, C. 1911: „Chomisch-physikalische Untersuchungen der dünischen Gewässer nebst Bemerkungen über ihre Bedeutung für unsere Auffassung der Temporalvariationen“. Intern. Rev. f. Hydrobiol., vol. 4, p. 251 u. 437 [Abschn. 2].

Bruxythaler, J., Prowízek, S., Wettstein, R. v. 1901: „Vorläufige Mitteilung über das Plankton des Attersees in Oberösterreich". Österr. Botan. Zeitschr., vol. 51, p. 73 [Abschn. 4, zitiert unter: BRunnthaler 1901 ].

Buffa, P. 1902: „Sulle condizioni fisiche e biologiche ai taluni laghi alpini del Trentino“. Atti Soc. Veneto-Trentina, ser. 2, vol. 4, fasc. 2, p. 5 (Padua). (Enthält Angaben über den Caldonazzosee, Lagoraisee, Lasessee, Piazzesee, Seraiasee und Stellunesee) [Abschn. 4].

Burckhardt, G. 1900: „Faunistische und systematische Studien über das Zooplankton der größeren Seen der Schweiz und ihrer Grenzgebiete". Revue Suisse, vol. 7, p. 353. (Enthält Angaben über den Bodensee) [Abschn. 4].

- 1910: „Wie das Plankton des Ritomsees durch die chemischen Eigentümlichkeiten des Wassers beeinflußt wird". Verhandlg. Schweiz. Naturf.-Gesellsch., 93. Jahresversammlg., vol. 1 Nr. 9 (Basel) [Abschn. 2].

Claus, C. 1877: „Zur Kenntnis des Baues und der Organisation der Polyphemiden“. Denkschr. Ak. Wiss. Wien, vol. 37. (Enthält p. 134 die Entomostrakenliste a. d. Traunsee) [Abschn. 4].

Cori, C. J. 1898: „Beitrag zur Fauna des Traunsees und seiner Umgebung“. In: Dr. F. Krackowitzer's „Geschichte der Stadt Gmunden“, Gmunden 1898. (Nicht zugänglich! Zitat aus LaNghans 1908) [Abschn. 1].

DADAY, E. v. 1891: „Beiträge zur mikroskopischen Süßwasserfauna Ungarns“. Természetraj̧zi Füzetek (Budapest), vol. 14, p. 107. (Enthält p. 113 Neusiedlersee!) [Abschn. 4].

DAнL, F. 1921: „Grundlagen einer ökologischen Tiergeographie“. Jena, Verlag G. FISCHER [Abschn. 2].

Dalla Torre, K. v. 1913: „Tirol, Vorarlberg und Liechtenstein“ in Junk's Naturführer. Verlag W. Junk, Berlin. (Enthält zahlreiche Angaben über die Seen des Gebietes) [Abschn. 4].

Douwe, van. 1908: „Zur Kenntnis der. Süßwassercopepoden Deutschlands“. Zoolog. Anz., vol. 32, p. 581. (Enthält Angaben über das Vorkommen von Canthocamptus hoferi und schmeili) [Abschn. 4].

- 1909: „Copepoda (Eucopepoda)“ in: Die Süßwasserfauna Deutschlands, herausgegeb. v. Brauer; 11. Heft. Verlag G. Fischer, Jena. (Enthält eine Angabe des Vorkommens von Heterocope weismanni im Starnbergersee) [Abschn. 4].

- 1917: „Zur Kenntnis der Süßwasserharpacticiden Deutschlands“. Zoolog. Anz., vol. 48, p. 277 [Abschn. 1]. 
Ekmax, Sv. 1904: „Die Phyllopoden, Cladoceren und freilebenden Copepoden der nordschwedischen Hochgebirge". Zool. Jahrb. 1. System. vol. 21, p. 137 [Abschn. 5].

Frech, Fr. 1903: „Über das Antlitz der Tiroler Zentralalpen“. Zeitschr. d. D. u. Ö. A. I., Jahrg. 1903 [Abschn. 4].

Fritz, G. 1922: „Das Plankton des Ammersees (Oberbayern) im Juli 1918“. Zool. Jahrb. f. Syst., vol. 44, p. 487 [Abschn. 4].

FugGer, E. 1890-1911: „Salzburgs Seen. I.-IX“. Mitteilg. (resellsch. Salzb. Landexkunde, vol. 30 (1890), vol. 31 (1891), vol. 33 (1893), vol. 35 (1895), vol. 39 (1899), vol. 43 (1903), vol. 45 (1905), vol. 48 (1908), vol. 51 (1911) [Abschn. 1 u. 4].

Garbivi, A. 1893: „Primi materiali per una monografia limnologica del lago di Garda“. Mem. Ac. Verona, vol. 69 [Abschn. 4].

- 1895: „Fauna limnetica e profonda dei Benaco“. Bollet. Mus. zooi. anat. Torino, vol. 10, Nr. 198 [Abschn. 4 u. 5].

- 1895: „Appunti di Carcinologia Veronese“. Mem. Ac. Verona, vol. 71 (ser. 3), fase. 1, p. 31. (Enthält alle Angaben der zwei zuerst zitierten Arbeiten über die Fauna des Gardasees) [Abschn. 4 u. 5].

Geistbeck, A. 1885: „Die Seen der Deutschen Alpen“. Mitteilg. Verein. f. Erdkunde, Leipzig 1884. (Enthält eine Darstellung des Seenphänomens im Gebirge und Vorland, Einteilung der Wasserbecken nach ihrer Entstehung, Tiefen- und Temperaturangaben und eine Diskussion über die Färbung der Gebirgsseen) [Abschn. 4].

Graeter, A. 19C3: „Die Copepoden der L'mgebung von Basel“. Revue Suisse Zool., vol. 11 [Abschn. b].

Haberbosch, P. 1917: „Über Süßwasserharpacticiden“. Archi: f. Hydrobiol., vol. 11, p. 593 [Abschn. 5].

Haempel, O. 1913: „Das Plankton des Chiemsees“. Arch. f. Hydrob., vol. 8, p. 319 [Abschn. 4].

- 1918: „Zur Kenntnis einiger Alpenseen mit besonderer Berücksichtigung ihrer biologischen und Fischerei-Yerhältnisse. I. Der Hallstättersee“. Internat. Rev. f. Hydrobiol., vel. 8, Heft 3, p. 225 [Abschn. 4].

- 1922: „II. Der Grundlsee“. Intern. Rev. f. Hydrob., vol. 10 [Abschn. 4].

Halbfass, W. 1913: „Der gegenwärtige Stand der Seenforschung“. Fortschr. naturwiss. Forschung (Abderhalden), rol. 6, p. 1, vol. 7, p. 1 u. 30, vol. 9, p. 1 [Abschn. 4].

Hartuaxy, E. 1913: „Der Schuppenbau der Tarntaler Berge am Westende der Hohen Tauern“. I. u. II. Teil (Schluß). Jahrb. Geolog. Reichsanstalt Wien, vol. 63, p. 207 u. 243 [Abschn. 1].

Hartmanx, O. 1914: „Beitrag zur Kenntnis der Cladocerenfauna der Umgebung von Graz mit einem Anhang über Cladocerenfunde in Obersteiermark". Mitteilg. naturw. Vereins Steiermark, Graz, vol. 51, p. 455. (Enthält Angaben über den Gaishornsee) [Abschnitt 4].

- 1916: „Über den Einfluß der chemischen Beschaffenheit des Mediums auf die Gestalt von Bosmina longirostris O. F. M."'. Archiv f. Entwicklungsmechanik (Leipzig), vol. 42, Heft 2, p. 208 [Abschn. 2].

- 1917: "Beitrag zur Kenntnis der Cladoceren- und Copepodenfauna Österreichs“. Archiv f. Hydrobiol., vol. 11, p. 82 [Abschn. 4].

Heller, C. 1871: „Untersuchungen über die Crustaceen Tirols“. Berichte d. naturwiss.medizin. Vereins Innsbruck, Jahrg. 1871. (Enthält Angaben über den Lansersee und Lansermoorsee, meist jedoch nicht über Seen als Fundorte) [Abschn. 4]. 
Heller, C. 1881: „Utber die Verbreitung der Tierwelt im Tiroler Hochgebirge. I. Abteilung". Sitzber. Ak. Wiss. Wien, vol. 83, I. Abtlg., Heft 1, p. 103 [Abschn. 1].

Heritsch, Fr. 1921: „Geologie von Steiermark“. Mittlg. Naturw. Vereins Steiermark, vol. 57 [Abschn. 3 u. 4].

Hofer, B. 1896: „Die Verbreitung der Tierwelt im Bodensee nebst vergleichenden Untersuchungen in einigen anderen Süßwasserbecken“. Schrift. Verein. f. Gesch. d. Bodensees $u$. Umgebung. Bodenseeforschungen Abschn. X, Lindau. (Diese Abhandlung wird vielfach unter der Zahl 1899 zitiert; sie enthält Angaben über den Achensee, Bodensee, Königssee, Starmbergersee, Walchensee) [Abschn. 4 u. 5].

Holdhaus, K. 1912: „Kritisches Verzeichnis der boreoalpinen Tierformen (Glazialrelikte) der mittel- und südeuropäischen Hochgebirge“. Annal. Naturhist. Mus. Wien, vol. 26, p. 399 [Abschn. 5].

Huber, G. 1906: „Monographische Studien im Gebiete der Montigglerseen (Südtirol) mit besonderer Berücksichtigung ihrer Biologie“. Archiv f. Hydrobiol., vol. 1, p. 1 u. 123 [Abschn. 4].

- 1907: „Der Kalterersee (Südtirol)“. Archiv f. Hydrobiol., vol. 2, p. 448 [Abschn. 4].

Hydrobiologische Kommission, Bericht der -. Verhandlg. d. Schweizeric ch. Naturforschenden Gesellschaft, Jahrg. 1918, I. Teil, p. 69 (Ritomsee-Untersuchungen) [Abschn. 2].

Імноғ, 0. 1885: „Faunistische Studien in 18 kleineren und größeren österreichischen Süßwasserbecken“. Sitzber. Ak. Wiss. Wien, vol. 91, p. 203 [Abschn. 4].

- 1887: „Úber die mikroskopische Tierwelt hochalpiner Seen“. Zool. Anz., vol. 10, p. 13 u. 33 [Abschn. 4].

- 1888: „Über das Calanidengenus Heterocope“. Zool. Anz., vol. 11, p. 447. (Enthält Angaben über das Auftreten von Heterocope im Chiemsee und Bodensee) [Abschn. 4].

- 1890: „Notizen über die pelagische Tierwelt der Seen in Kärnten und in Krain“. Zool. Anz., 13. Jahrg. Nr. 325-352, p. 261, 347 u. 372 [Abschn. 4].

- 1890: „Notizen über die Süßwassercalaniden“. Zool Anz, vol. 13, p. 654 [Abschn. 4 u. 5].

Jä́cke, J. 1916: „Aus den Niederen Tauern“. Zeitschrift d. D. u. Ö. A.V., vol. 47, p. 37 bis 40 [Abschn. 1].

KeIlHack, L. 1909: „Phyllopoda“ in: die Süßwasserfauna Deutschlands, herausgeg. v. Brauer, Heft 10; Verlag G. Fischer, Jena [Abschn. 5].

Keissler, K. v. 1900: „Das Plankton des Unteren Lunzersees in Niederösterreich“. Verhandlg. zool. bot. Gesellsch. Wien, vol. 50, p. 541 [Abschn. 4].

- 1901: "Notiz über das Plankton des Aber- oder Wolfgangsees in Salzburg“. Verhandlg. zool. bot. Gesellsch. Wien, vol. 51 (Heft 6), p. 401 [Abschn. 4].

- 1901: Zur Kenntnis des Planktons des Attersees in Oberösterreich“. Verhandlg. zool. bot. Gesellch. Wien, vol. 51 (Heft 6), p. 392 [Abschn. 4].

- 1902: „Kurze Mitteilungen über das Phytoplankton des Nussensees bei Ischl in Oberösterreich“. Österr. botan. Zeitschr., vol. 52, p. 6. (Enthält Zooplanktonangaben!) [Abschn. 4].

- 1902: „Ưber das Plankton des Aber- oder Wolfgangsees in Salzburg“. Verhandlg. zool. bot. Gesellsch. Wien, vol. 52, p. 305 [Abschn. 4].

- 1902: „Zur Kenntnis des Planktons des Altausseersees in Steiermark“. Verhandlg. zool botan. Gesellsch. Wien, vol. 52, p. 706 [Abschn. 4]. 
KeissLer, K. v. 1903: „Ửber das Plankton des Hallstättersees in Oberösterreich“. Verhandl. zool. botan. Gesellsch. Wien, vol. 53, p. 338 [Abschn. 4].

- 1904: „Einige Planktonfänge aus dem Brennsee bei Feld in Kärnten“. Österr. botan. Zeitschr., vol. 54, p. 58 [Abschn. 4].

- 1904: „Das Plankton des Millstättersees in Kürnten“. Österr. botan. Zeitschr., vol. 54, p. 218 [Abschn. 4].

- 1905: „Mitteilungen über das Plankton des Ossiachersees in Kärnten“. Österr. botan. Zeitschr., vol. 55, p. 101 u. 108 [Abschn. 4].

- 1906: „Beitrag zur Kenntnis des Planktons einiger kleinerer Seen in Kärnten“. Österr. botan. Zeitschr., vol. 56, p. 53. (Enthält Angaben über den Faakersee, Jeserzsee, Keutschachersee, Klopeinersee, Worstnigsee) [Abschn. 4].

- 1906: "Planktonstudien über den Wörthersee in Kärnten“. Österr. botan. Zeitschr., vol. 56, p. 195 [Abschn. 4].

- 1906: „Notiz über das Augustplankton des Gardasees“. Österr. botan. Zeitschr., vol. 56, p. 414 [Abschn. 4].

- 1907: „Planktonstudien über einige kleinere Seen des Salzkammergutes". Österr. botan. Zeitschr., vol. 57, p. 51. (Enthält Angaben über den Altausseersee, Grundlsee, Ödensee, Offensee, Röthelsee) [Abschn. 4].

- 1907: „Über das Phytoplankton des Traunsees“. Österr. botan. Zeitschr., Jahrg. 1907, Nr. 4, p. 146. (Enthält auch Zooplanktonangaben!) [Abschn. 4].

- 1910 „Planktonuntersuchungen in einigen Seen der Julischen Alpen in Krain“. Archiv f. Hydrobiol., vol. 5, p. 351. (Enthält Angaben über Veldessee, Wocheinersee) [Abschn. 4].

- 1910 „Untersuchungen über die Periodizität des Phytoplanktons des Leopoldsteinersees in Steiermark etc.". Archiv f. Hydrobiol., vol. 6, p. 480. (Enthält auch Zooplanhtonangaben) [Abschn. 4].

Klejber, O. 1911: „Die Tierwelt des Moorgebietes von Junghclz im südlichen Schwarzwald“. Archiv f. Naturg., Jahrg. 77, 1. Band, 3. Supplementheft, p. 1 [Abschn. 5?.

Klunzinger, B. 1907: „Ergebnisse der neueren Bodenseeforschungen“. Archiv f. Hydrobiol., vol. 2, p. 97. (Referat u. Literaturangaben!) [Abschn. 4].

Langhaxs, V. 1905: „UUber das Zooplankton der Julischen Alpenseen“. Zeitschr. Lotos, Prag. (Enthält Raiblersee) [Abschn. 4].

- 1908: „Das Plankton des Traunsees in Oberösterreich“. Zeitschr. Lotos, Prag, vol. 56, p. 209 u. 255 [Abschn.4].

- 1911: "Cladoceren aus dem Salzkammergut". Zeitschr. Lotos, Prag, vol. 57, p. 172 [Abschn. 4].

I.ARGAIOLLI, V. 1898: "Materiali per una monografia biolimnologica del Trentino“. Revista „Tridentum“, anno I, fasc. 1, p. 64. (Enthält Angaben über den Caldonazzosee, Gardasee, Ledrosee, Levicosee, Loppiosee, Santa Massenzasee, Seraiasee, Toblinosee und Piazzesee) [Abschn. 4].

- 1906: "Ricerche biolimnologiche sui laghi trentini. I. Il lago di Lavarone". Rivista mensile di pesca anno VIII, Nr. 1-3 [Abschn. 4].

- 1906: "Diaphanosoma brachyurum LIÉvin var. tridentinum mihi". Archiv f. Hydrobiol., vol. 1, p. 428 [Abschn. 4].

- 1906: „Ricerche biolimnologiche sui laghi trentini. II. Il lago di Terlago“. Atti Acc. Veneto-trent. istrian., vol. 3, fasc. 1, p. 35 [Abschn. 4].

- 1906/07: „Ricerche biolimnologiche sui laghi Trentini. III. Il lago Santo“. Rivista „Trentinum", anno 1906, fase. 9-10, Trento 1907 [Abschn. 4]. 
IARGAOLLJ, V. 19(17: ., Ricerche biolimnologiche sui laghi trentini. IY. Il lago di Tovel (Bacino del Noce) “. Atti Acc. scient. Veneto-Trentino-istriana, anno 4, fase. 1; Padova 1907 [Abschn. 4].

- 1908/09: "Ricerche biolimnologiche sui laghi trentini. V. Il. lago di Cei“. Tridentum, anno 1908, fasc. 8. Trento 1909 [Abschn. 4].

1910: "Ricerche biolimnologiche sui laghi trentini. VI. Il lago di Loppio“. Rivista mensile di pesca ed idrobiologia anno V (XII), 1910, Nr. 7-9, p. 193 (Pavia) [Abschn. 4].

Lauterborn, R. 1913: „Süßwasserfauna“ in: Handbuch d. Naturwissenschaften, vol. 9, p. 861-920. Verlag G. Fischer, Jena [Abschn. 5].

LEuze, M. 1913: „Bythotrephes longimanus. Ein newer Fundort“. Archiv f. Hydrobiol., vol. 8, p. 152 [Abschn. 4].

LilljeborG, W. 1901: „Synopsis specierum huc usque in suecia observatorum generis Cyclepis". K. Svenska Vet. Ak. Handling. vol. 35 (Stockholm) [Ab:chn. 5].

LINT, G. M. DE 1922: „Untersuchungen über Planktoncopepoden in Niederländischen Gewässern". Internat. Rev. f. Hydrobiol, vol. 10, p. 76 [Abschn. 5].

Lorenzi, A. 1896: „Una visita al laghetto di Cima Corso (Ampezzo)“. In Alto, cronaca soc. alpina friulina, anno 7. (Nicht zugänglich!). (Ebenso in anno 10 [1899]).

Lorenz-LiburnaU, v. 1898: „Der Hallstättersee, eine limnologische Studie“. Mittlg. geograph. Gesellsch. Wien, vol. 41, p. 1-218 [Abschn. 4].

Micoletzky, H. 1910/11: „Zur Kenntnis des Faistenauer Hintersees bei Salzburg, mit besonderer Berücksichtigung faunistischer und fischereilicher Verhältnisse“. Internat. Revue f. Hydrobiol., vol. 3, p. 5C6 [Abschn. 4].

- 1912: „Beiträge zur Kenntnis der Ufer- und Grundfauna einiger Seen Salzburgs sowie des Attersees“. Zool. Jahrb. f. Syst., vol. 33, p. 421 [Abschn. 4].

- 1913: „Zur Kenntnis des Faistenauer Hintersees bei Salzburg. Nachtrag zur Litoralfauna". Internat. Rev. f. Hydrobiol. Biolog. Supplem. VI. ser., p. 1 [Abschn. 4].

Moniez, R. 1887: „Pêches de M. Dollfus dans les laes de l'Engadin et du Tyrol“. Fuille d. jeun. natur., année 18, Nr. 205, p. 5, sub: communications (nicht année 17, 1886; enthält Angaben über den Haidersee) [Abschn. 4].

MoNт, R. 1906: „Recherches sur quelques lacs du massif du Ruitor“. Annal. biolog. lacustr., vol. 1 [Abschn. 2].

- 1910: „La vita negli alti laghi alpini“. Natura, rivista mensile di sci. nat. Milano, vol. 1, fasc. 5, p. 153. (In dieser Arbeit werden unter anderen der Foscagnosee und Frä̈lesee genannt; laut einer schriftl. Mittlg. von R. Mosti sind jedoch die Bearbeitungen der Copepoden und Cladoceren dieser Seen noch nicht abgeschlossen) [Abschn. 4].

Müllner, J. 1896: „Erläuterungen zur ersten Lieferung des österreichischen Seenatlas: Die Seen des Salzkammergutes“. Geograph. Abhandlg. (Penck), vol. 6, Heft 1 (HölzL, Wien) [Abschn. 4].

- 1905: „Die Seen des unteren Inntales in der Umgebung von Rattenberg und Kufstein". Zeitschr. d. Ferdinandeums f. Tirol u. Vorarlberg, Innsbruck, 3. Folge, Heft 49, p. 139. (Enthält topographische und hydrographische Angaben über den Hechtsee, Hintersteinersee, die Reintalerseen [Berglsteinersee, Krummsee, Rrintalersee] und Tiersee) [Abschn. 1 u. 4].

Naumane, E. 1918: „Über die natürliche Nahrung der limnischen Zooplanktons. Ein Beitrag zur Kenntnis des Stoffhaushaltes im Süßwasser". Lunds UniversitetsÅsskrift N.F. Avd. 2, vol. 14, Nr. 31 [Abschn. 1]. 
Pavesi, P. 1879: .,Nuova serie di ricerche della fauna pelagica nei laghi italiani“. Rendiconti R. Ist. Lombardo (Milano) ser 2, vol. 12, p. 474 [Abschn. 4].

- 1879: „Ulteriori studi sulla fauna pelagica dei laghi italiani“. Rendic. R. Ist. Lombardo (Milano) ser. 2, vol. 12, p. 688. (Die zwei Abhandlungen enthalten Angaben über den Idrosee u. Iseosee) [Abschn. 4].

- 1882: „Escursione zoologica al lago di Toblino“. Atti soc. italian. sei. nat. (Milano), vol. 25, p. 142 [Abschn.4].

- 1884: "Altra serie di ricerche e studi sulla fauna pelagica dei laghi italiani“. Atti Soc. Veneto-Trentina (Padova), vol. 8, p. 340. (Enthält Angaben über den Alleghesee, Caldonazzosee, Gardasee, Ledrosee, Levicosee, Loppiosee, Revinesee, Santa Crocesee) [Abschn. 4].

- 1889: "Notes physiques et biologiques sur trois petits lacs du bassin tessinois". Arch. sci. phys. nat., vol. 22 [Absehn. 2].

Pesta, O. 1911: „Zur Fauna einiger Gebirgsseen in Kärnten und Tirol“. Verhandlg. zool. botan. Gesellsch. Wien, vol. 61, p. 117. (Enthält Angaben über den Fresenhalssee, Oberen und Unteren Mühldorfersee, Schlickersee, Speiksee) [Abschn. 4 u. 5.

- 1912: „Hochgebirgsseen in Tirol und ihre Fauna. I. Beitrag“. Verhandlg. zool. botan. Gesellsch. Wien, vol. 62, p. 158. (Enthält Angaben über den Großen und Kleinen Bödensee, Mutterbergersee, Unt. Seebisee) [Abschn. 4].

- 1914: „Hochgebirgsseen in Tirol und ihre Fauna. II. Beitrag“. Verhandlg. zool. botan. Gesellsch. Wien, vol. 64, p. 210. (Enthält Angaben über den Boêsee, Kreuzjochsee) [Abschn. 4].

- 1915: „Hochgebirgsseen in Tirol und ihre Fauna. III. Beitrag“. Verhandlg. zool. botan. Gesellsch. Wien, vol. 65, p. 227. (Enthält Angaben über den Unt. Plenderlesee) [Abschn. 4].

- 1918: „Über einige Fragen aus der neueren Planktonforschung“. (Vortrag). Verhandlg. zool. botan. Ges. Wien, vol. 68, p. (269).

Richard, J. 1896: „Sur la faune pélagique du Tegernsee“. Zool. Anz., vol. 19, p. 28. (Enthält auch Kreuthsee) [Abschn. 4].

RichteR, E. 1897: „Seenstudien. Erläuterungen zur zweiten Lieferung des Atlas der österreichischen Alpenseen". Geograph. Abhandlg. (Penck), Wien, vol. 6, Heft 2, p. 121-191 [Abschn. 4].

RüHE, F. E. 1912: „Die Bosminenfauna zusammenhängender Seengebiete“. Archiv f. Hydrobiol., vol. 7, p. 475 [Abschn. 1].

- 1915: „Beitrag zur Biologie der Bosminen des Salzkammergutes“. Archiv f. Hydrobiol., vol. 10, p. 77. (Bezieht sich auf den Grundlsee, Hallstättersee u. Traunsee) [Abschn. 4].

SARS, G. O. 1913: „An account of the Crustacea of Norway. Copepoda Cyclopoida". Bergen 1913, vol. 6, parts 3 and 4 [Abschn. 1 u. 5].

- 1914: Dasselbe; Bergen 1914, vol. 6, parts 5 and 6 [Abschn. 5].

Schattauer, J. 1914: „Das Gebiet des Giglachsees in den Niederen Tauern“. Nittlg. D. u. Ö. A.V. 1914, Nr. 8, p. 113-116 [Abschn. 1].

Scileffett, E. 1922: „Das Zooplankton des Chiemsees im Hochwasserjahr 1920“. Archiv f. Naturgesch., 88. Jahrg., Abtlg. 4, Heft 9, p. 1 [Abschn. 4].

Schmeil, O. 1892: „Deutschlands freilebende Süßwassercopepoden. I. Cyclopidae“. - Biblioth. Zool. 11. Heft [Abschn. 5].

- 1893: „Copəpoden des Rhätikongebirges“. Abhandlg. Naturf. Gesellsch. Halle, vol. 19, p. 1 (am Titelblatt 1893-1895; enthält Berichtigungen der Bestimmungen von Zschокке und Poppe) [Abschn. 4]. 
SExy. 1. 1890: „Escursione zoologica a due laghi friulani“. Boll. entom. soc. italian. (Firenze), rol.22, p. 93. (Enthält Angaben über den Cavazzosee u. San Danielesee) [Abschn. 4].

Stemmane, G. 1906: "Geologische Probleme des Alpengebirges“. Keitschr. d. D. u. Ö. A.V., vol 37, p. 1 [Abschn. 4].

STEvir, $\Lambda .1897$ : "Liste aller bisher in Kärnten gefundenen Cladoceren und Copepoden“. (arinthia (Klagenfurt), Il., Nr. 4 [Abschn. 4 u. 5].

- 1897: „Ein Beitrag zux Kenntnis der Cladoceren- und Copepodenfauna Kärntens“. Verhandlg. zool. botan. Gesellsch. Wien, vol. 47, p. 1-49. (Enthält gegenüber der vorstehenden Pablikation keine neuen Funde) [Abschn. 4 u. 5].

- 1901: „Die Entomostrakenfauna der alten Donau bei Wien“. Zool. Jahrb. Syst., vol. 15. (Enthält auf p. 128-142 Angaben über den Attersee, Augstwiesensee, Chiemsee, Lunzer Untersee, Mondsee, Presseckersee, Schliersec) [Abschn. 4 u. 5$]$.

- 1910: „Planktonkunde“. Verlag Teubner [Abschn. 2 u. 3].

Stingelin, Th. 1895: „Die Cladoceren der Umgebung von Basel“. Revue Suisse Zool. vol. 3, p. 161—274 [Abschn. 1].

- 1905: „Unser heutiges Wissen über die Systematik und die geographische Vorbereitung der Cladoceren". C. R. 6. Congrès internat. Zool. (Berne 1904), p. 533. (Nicht zugänglich!)

- 1906: „Neue Beiträge zur Kenntnis der Cladocorenfauna der Schweiz“. Revue Suisse Zool., vol. 14, p. 317 [Abschn. 4 u. 5].

- 1908: "Phyllopodes". Catalogue d. Invertebr. d. 1. Suisse. Mus. Hist. Nat. Genève [Abschn. 3 u. 5].

Thiébaud, M. 1915: „Copepodes“. Catalogue d. Invertebr. d. 1. Suisse. Mus. Hist. Nat. Genève [Abschn. 3 u. 5].

Thienemann, A. 1913: „Die Faktoren, welche die Verbreitung der Süßwasserorganismen regeln". Archiv f. Hydrobiol., vol. 8, p. 267 [Abschn. 2].

- 1922: „Biologische Seentypen und die Gründung einer hydrobiolog. Anstalt am Bodensee“. Archiv f. Hydrobiol., vol. 13, Heft 3, p. 347 [Abschn. 1].

Tollinger, A.: 1911: „Die geographische Verbreitung der Diaptomiden“. Zool. Jahrb. Syst., vol. 30, p. 1 [Abschn. 5].

Wallmann, H. 1868: „Die Seen der Alpen“. Zeitschr. d. D.Ö.A.V., vol. 4 (Wien, GEROLD) [Abschn. 4].

WeIth, W. 1880: „Chemische Untersuchungen der schweizerischen Gewässer mit Rücksicht auf deren Fauna“. Internat. Fischereiausstellung, Berlin 1880; Katalog der Schweizer Beteiligung, p. 96 [Abschn. 2].

Wönt, H. 1918: „Der Klafferkessel in den Schladminger Tauern“. Zeitschr. d. D. u. Ö. A.V., vol. 49, p. 101-124 [Abschn. 1].

Wolterecr, 1906: „Mitteilungen aus der biologischen Station in Lunz“. Biol. Zentralblatt, vol. 26, p. 463 [Abschn. 4].

ZACHARIAS, O. 1902: „Zur biologischen Charakteristik des Schwarzsees bei Kitzbühel in Tirol". Biol. Zentralbl., vol. 22, p. 701 [Abschn. 4].

- 1903: „Mitteilungen über das Plankton des Achensees in Tirol“. Biolog. Zentralblatt, vol. 23, p. 162 [Abschn. 4].

- 1905: „Hydrobiologische und fischereiwirtschaftliche Beobachtungen an einigen Seen der Schweiz und Italiens". Forschgsber. biol. Stat. Plön, vol. 12, p. 169. (Enthält p. 284 Angaben über den Starnbergersee.) [Abschn. 4].

- 1906: „Zur Biologie und Ökologie von Polyphemus pediculus (Linné). Zool. Anz., vol. 30 [Abschn. 5]. 
Zschoкке, Fr. 1890: (1893 am Titelblatt!) „Faunistische Studien an Gebirgsseen". Verhandlg. Naturf. Ges. Basel, vol. 9, p. 1. (Enthält Angaben über den Garschinasee, Lünersee, Partnunersee, Tilisunasee.) [Abschn. 4].

- 1891: (1893 am Titelblatt!) „Die zweite zoologische Exkursion an die Seen des Rhütikon". Verhandlg. Naturf. Ges. Basel, vol. 9, p. 425. (Enthält weitere Angaben über die vorstehend genannten Seen.) [Abschn. 4].

- 1894: (1897 am Titelblatt!) „Die Fauna hochgelegener Gebirgsseen". Verhandlg. Naturf. Ges. Basel, vol 11, p. 36. (Enthält Angaben über den Gafiensee und Todtalpsee.) [Abschn. 1, 4 u. 5].

- 1900: "Die Tierwelt der Hochgebirgsseen“. N. Denkschrift. schweiz. Ges. f. Naturw., vol. 37 (vol. 7); Zürich [Abschn. 2 u. 4].

Bei der Bearbeitung des 4. Abschnittes dieser "Studien" wurden folgende Landkartenwerke und Ausgaben benützt:

Frech, F. 1895-1898: Geologische Karte der Radstätter Tauern.

HAuer, R. ․ 1867-68: Geologische Übersichtskarte der österreich. Nonarchie, Die: - Ostalpen, Blatt 5 u. 6.

Penck und Richter 1895-1896: Atlas der österreich. Alpenseen. I. Lieferung: Die Seen des Salzkammergutes. II. Lieferung: Seen von Kärnten, Krain und Südtirol. Beilagen zu den Geogr. Abhandlg., vol. 6, Heft 1. Hölzes (Wien). SonNklar 1866: Die Hohen Tauern.

Spezialkarten der österr.-ung. Monarchie und der angrenzenden Teile des Auslandes. Ersch. im Verlage des militär-geogr. Institutes Wien.

Kartenbeilagen zur Zeitschrift des D. u. Ö. A.V.

Ferner sei verwiesen auf zwei schwer zugängliche Aufsätze allgemeinen Inhaltes, nämlich:

Langhans, V. 1909: „Planktonprobleme". Naturwiss. Zeitschrift Lotos (Prag), vol 57, p. 172.

Brenм, V. 1914-1916 „Probleme der modernen Planktonforschung“. I. Teil: Einleitung. II. Teil: Glazialbiologie. III. Teil: Das Nannoplankton. Jahresberichte d. Staatsgymnasiums in Eger (Böhmen). Eger 1914, 1915 u. 1916. 

Archiv f.

laf.in.

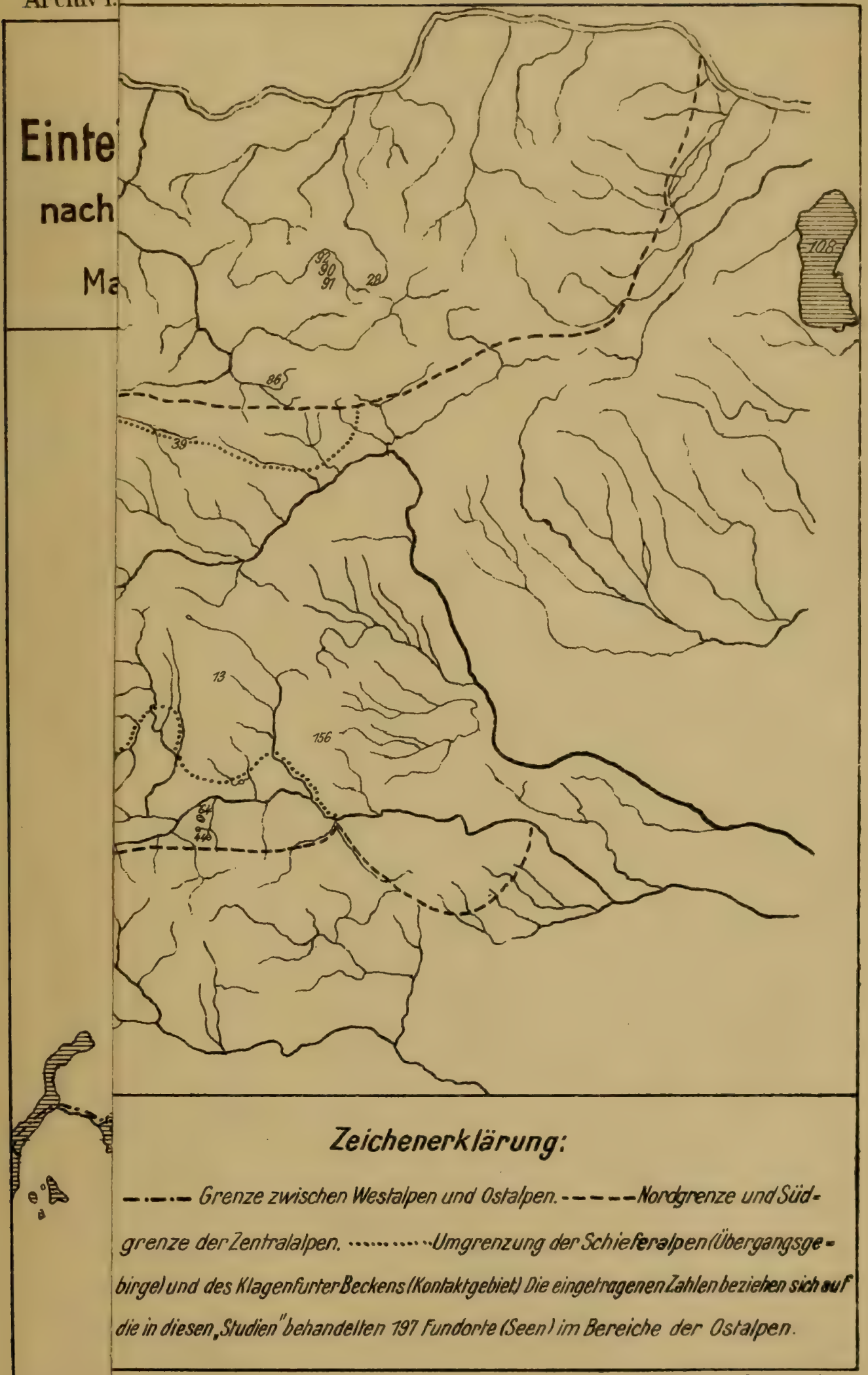

H. Wriscinnen: Stuttgary 

Archir f. Hrdeobiologie Suppl.18d. III.

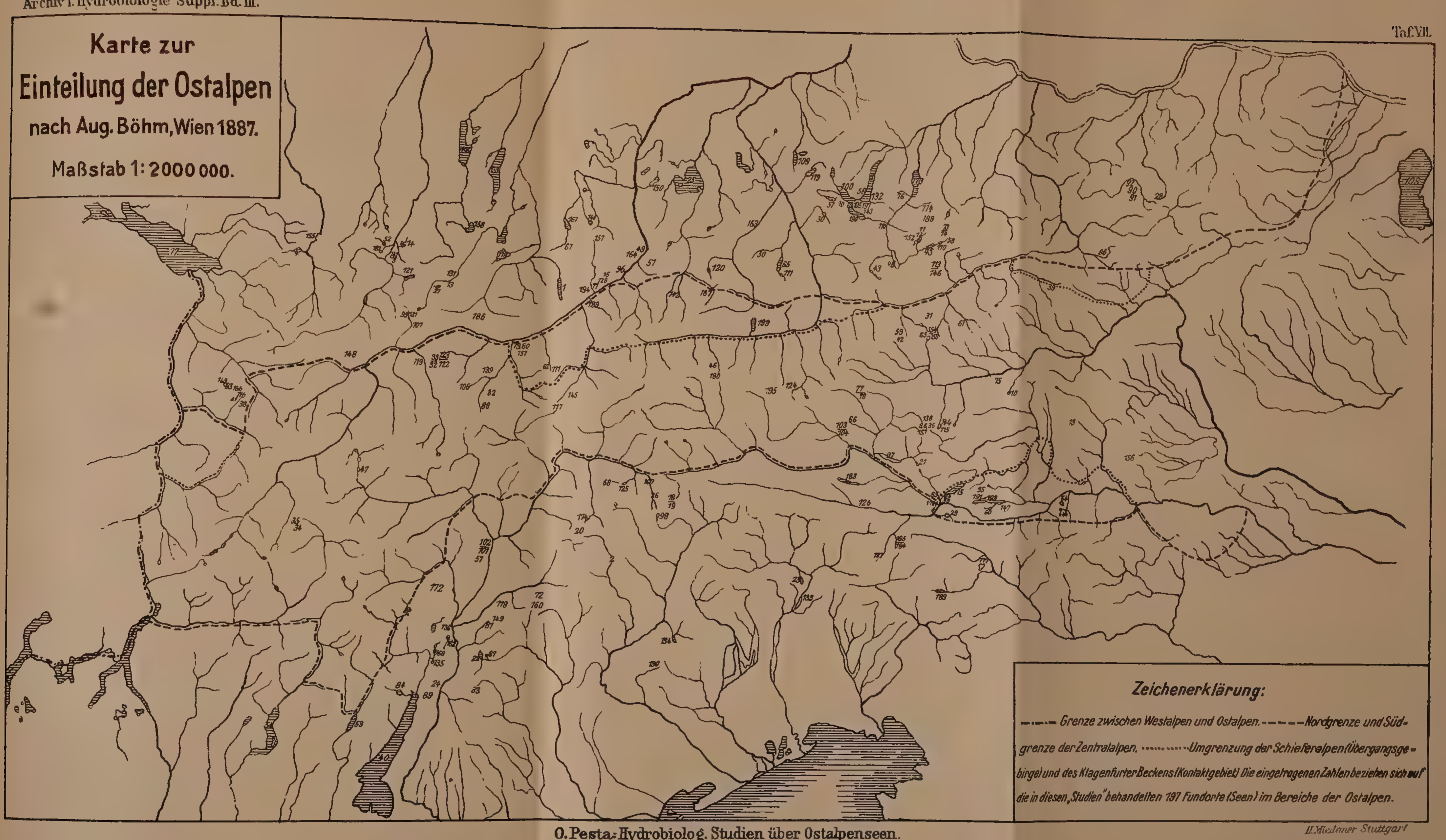



Taf. VIII

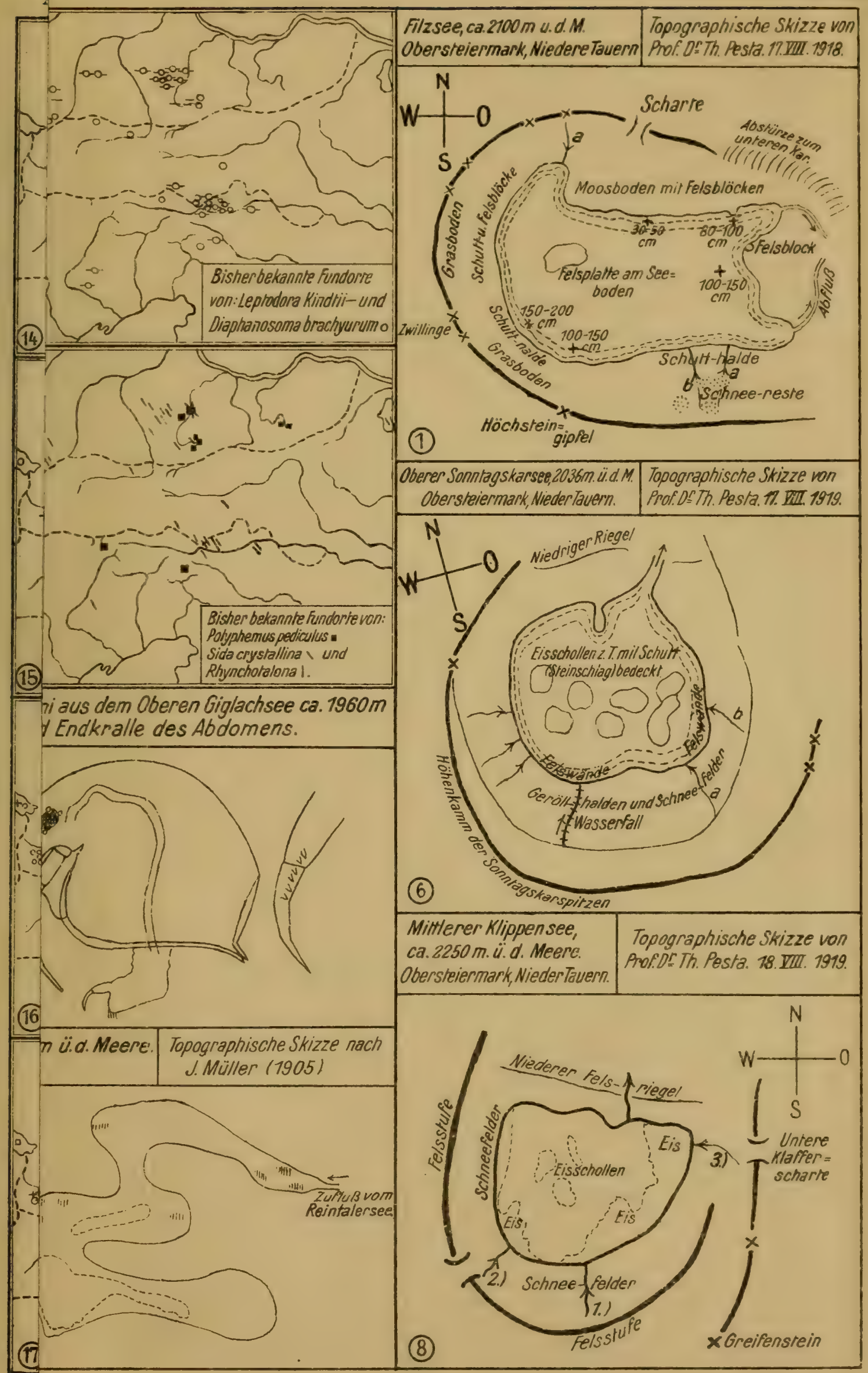

E.Mfiedonen, Stuttgiert 




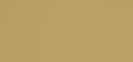

$$
\text { . }
$$

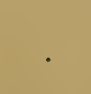




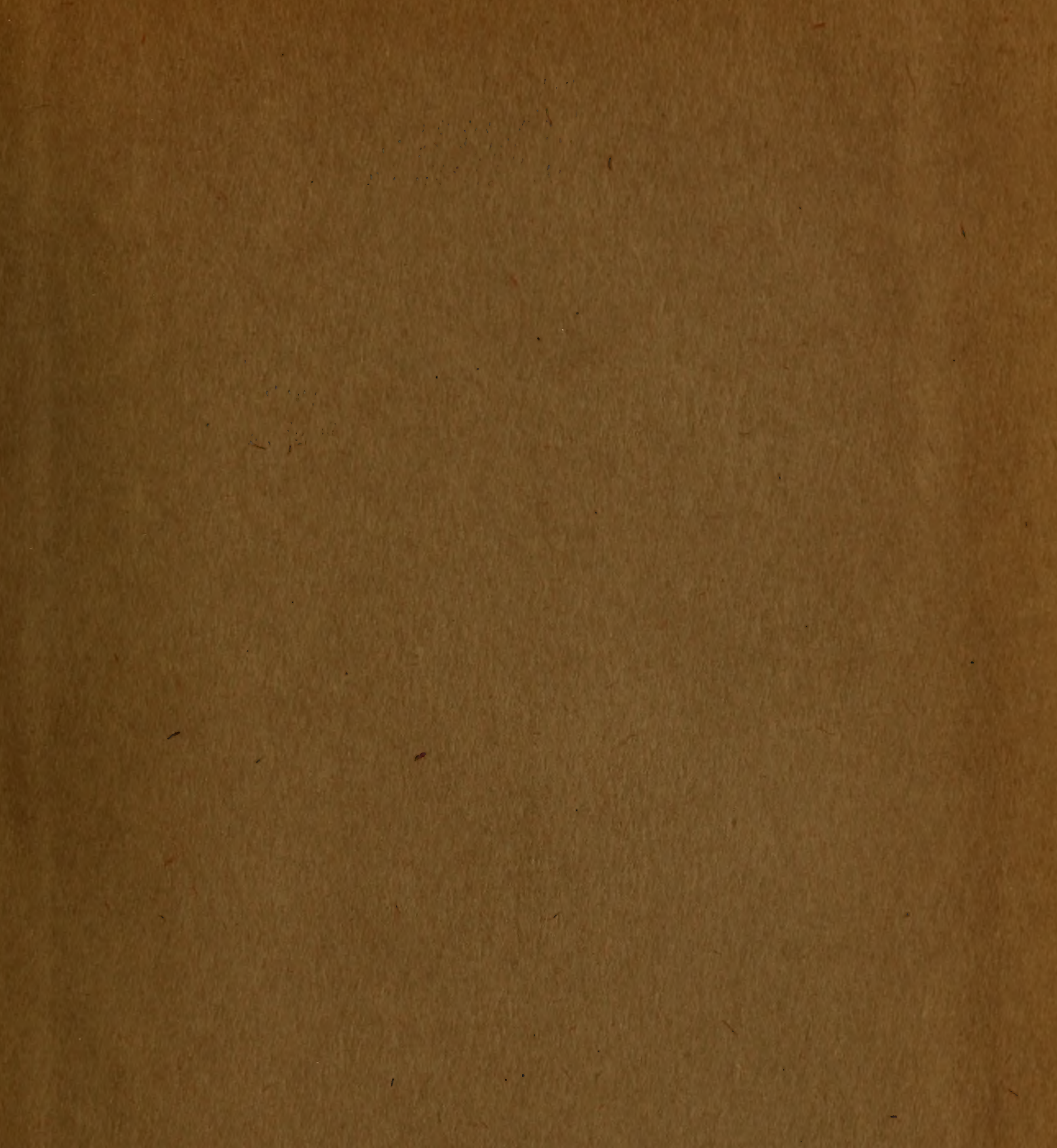



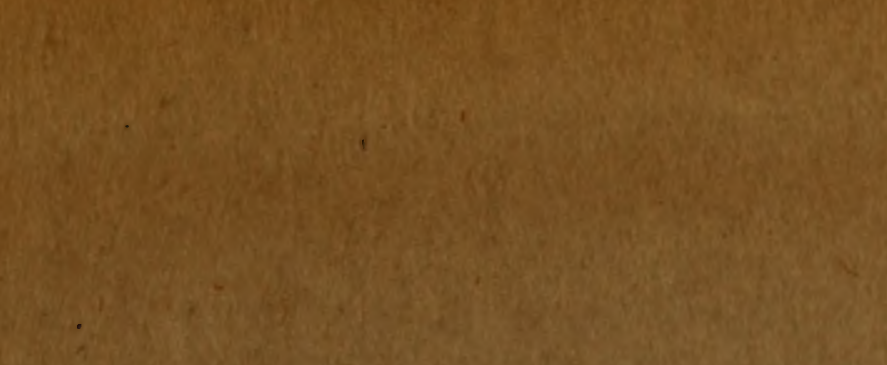
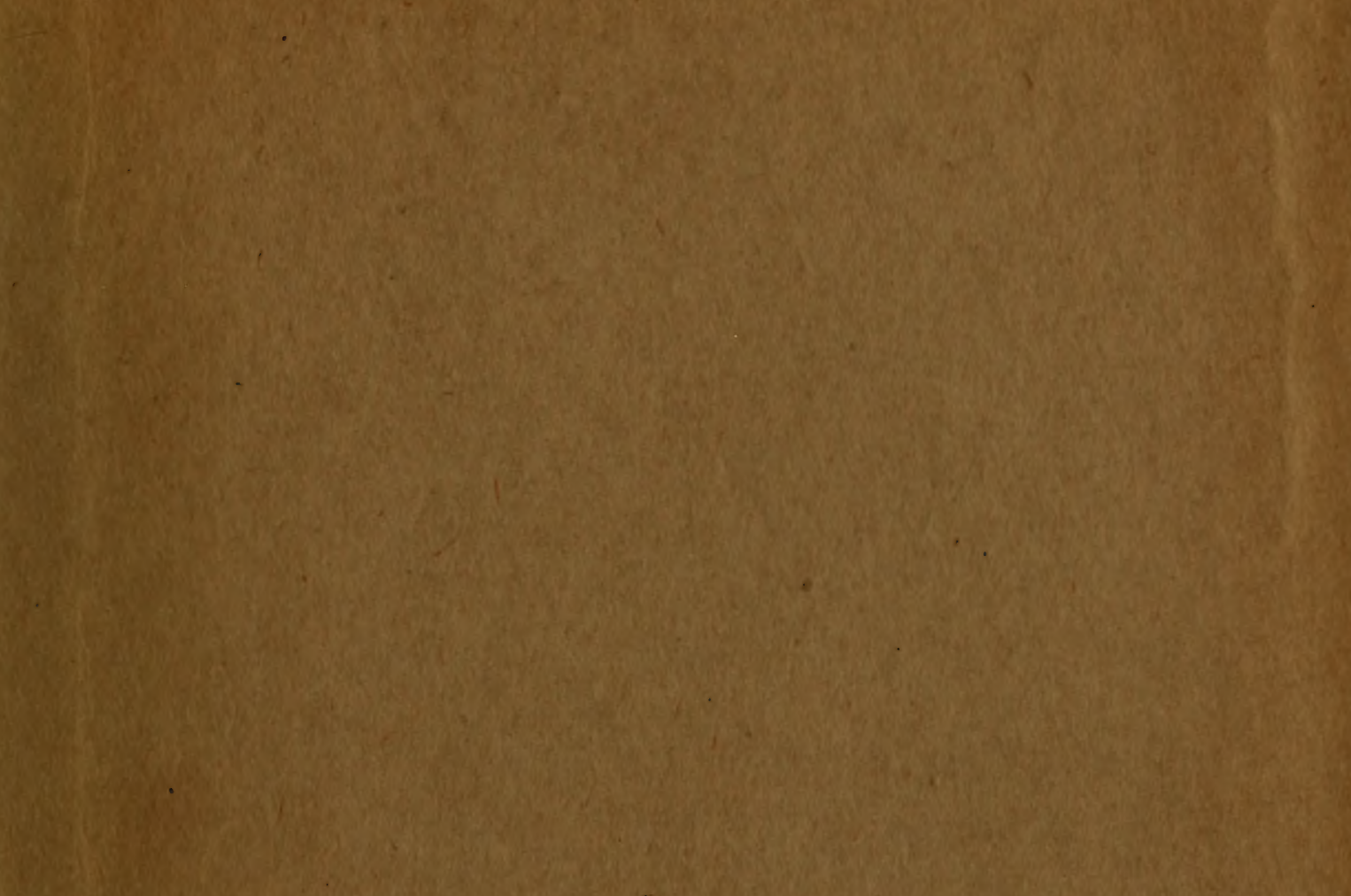

- 53
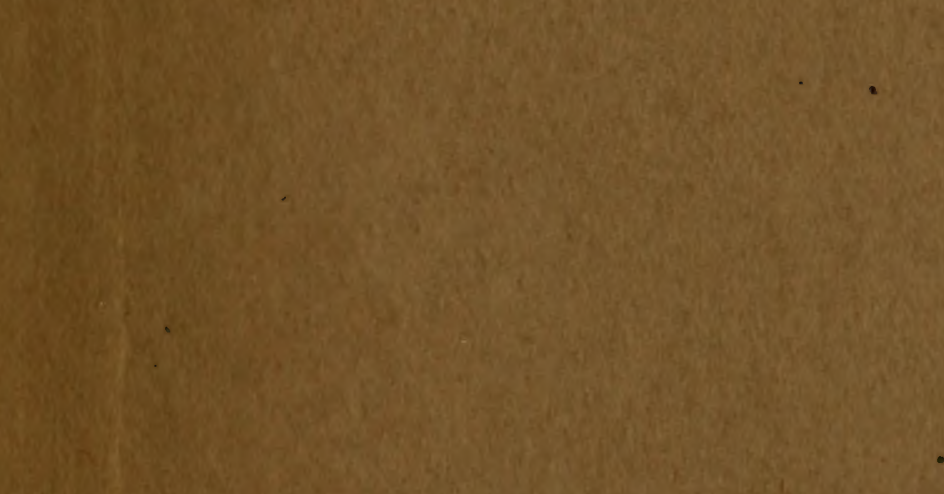

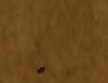
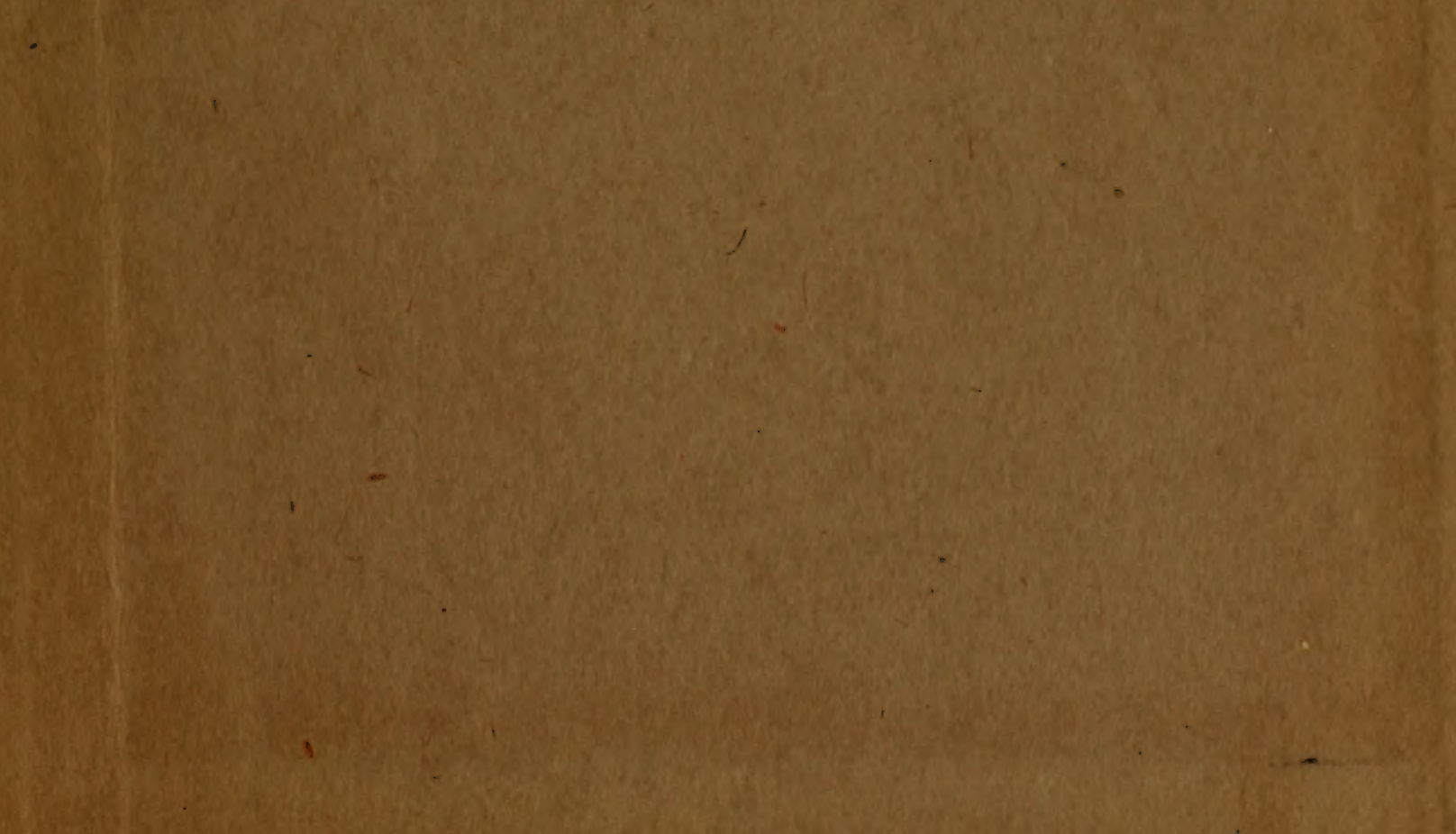


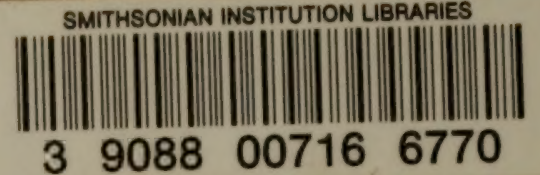

H. 